\title{
OPTICAL BIONANOSENSORS FOR NEUROTRANSMITTER DETECTION
}

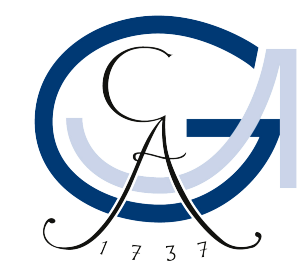

DISSERTATION

For the award of the degree

„Doctor rerum naturalium “

of the Georg-August-University Göttingen

within the doctoral program

"Physics of Biological and Complex Systems"

of the Georg-August University School of Science (GAUSS)

submitted by

Elena Alexandra Polo

from Moscow / Russia

Göttingen, 2018 



\section{Thesis Committee}

Dr. Sebastian Kruss

Institute of Physical Chemistry

University of Göttingen

Prof. Dr. Jörg Enderlein

Biophysics / Complex Systems at the III. Institute of Physics

University of Göttingen

Prof. Dr. Claudia Höbartner

Institute of Organic Chemistry

University of Göttingen

\section{Members of the Examination Board}

\section{Referee}

Dr. Sebastian Kruss, Institute of Physical Chemistry, University of Göttingen

\section{2nd Referee}

Prof. Dr. Jörg Enderlein, Biophysics / Complex Systems at the III. Institute of Physics, University of Göttingen

\section{Further Members of the Examination Board}

Prof. Dr. Sarah Köster, Institute for X-Ray Physics, University of Göttingen

Prof. Dr. Martin Suhm, Institute of Physical Chemistry, University of Göttingen

Prof. Dr. Ricardo Mata, Computational Chemistry and Biochemistry Group, University of Göttingen

Dr. Florian Rehfeldt, Third Institute of Physics, University of Göttingen

Date of oral examination: 
Hiermit erkläre ich, Elena Alexandra Polo, die Dissertation mit dem Titel Optical bionanosensors for neurotransmitter detection eigenständig angefertigt und keine anderen als die von mir angegebenen Quellen und Hilfsmittel verwendet zu haben.

Göttingen, den 29.03.2018

Elena Alexandra Polo 
I, Elena Alexandra Polo, hereby certify that my doctoral thesis entitled Optical bionanosensors for neurotransmitter detection has been written independently and with no other sources and aids than quoted.

Göttingen, 29.03.2018

Elena Alexandra Polo 

To my family

New ideas pass through three stages: 1) It can't be done.

2) It probably can be done, but it's not worth doing. 3) I knew it was a good idea all along!

Arthur C. Clarke 



\section{Contents}

\begin{tabular}{lll}
\hline Abstract & IX
\end{tabular}

\begin{tabular}{lll}
\hline & Introduction & 1
\end{tabular}

1.1 Chemical signaling in the brain . . . . . . . . . . . . . . . . . . . 1

1.1.1 Requirements for spatiotemporal neurotransmitter sensors . . . . . . 2

1.2 Techniques to determine local neurotransmitter concentrations . . . . . . . . 6

1.2.1 Electrochemical sensing . . . . . . . . . . . . . . . . 6

1.2 .2 Optical sensing . . . . . . . . . . . . . . . . . . . . . . . . 9 9

1.2.3 Optical sensing based on single-walled carbon nanotubes (SWCNTs) 12

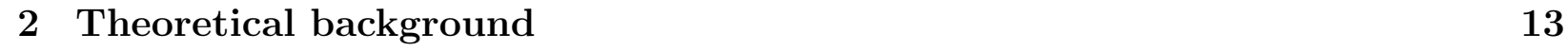

$2.1 \quad$ Single-walled carbon nanotubes (SWCNTs) . . . . . . . . . . . . . . . 13

$2.1 .1 \quad$ Synthesis of SWCNTs . . . . . . . . . . . . . . . . . . . . 13

$2.1 .2 \quad$ Structure and geometry of SWCNTs . . . . . . . . . . . . . . . 15

2.1 .3 Photophysical properties of SWCNTs . . . . . . . . . . . . . . 17

2.1 .4 Raman spectroscopy of SWCNTs . . . . . . . . . . . . . . . . . 23

2.2 Functionalization strategies $\ldots \ldots \ldots \ldots \ldots \ldots$

$2.2 .1 \quad$ SWCNT-based sensor applications . . . . . . . . . . . . . . . . 28

$2.2 .2 \quad$ Functionalization and selectivity . . . . . . . . . . . . . . . . . . . . 29

2.2 .3 Sensitivity and detection limits . . . . . . . . . . . . . . . 30

2.2 .4 The integrin binding motif RGD . . . . . . . . . . . . . . . . . 34

2.3 Near-infrared fluorophores for biomedical imaging . . . . . . . . . . . . . . . 37

$2.3 .1 \quad$ Scattering in biological tissues . . . . . . . . . . . . . . . 37

2.3 .2 Advantages of the near Infrared region $\ldots \ldots \ldots$. . . . . . . . . 38

$2.3 .3 \quad$ Egyptian Blue as nIR emitter . . . . . . . . . . . . . . . . . . . . . . 41

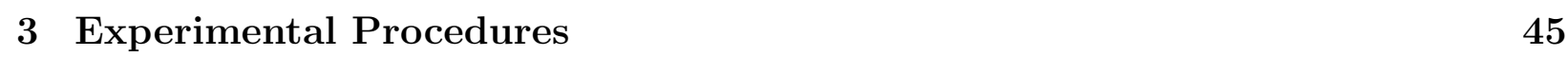

3.1 Materials . . . . . . . . . . . . . . . . . . . . . . . . . 45

3.1 .1 Chemicals . . . . . . . . . . . . . . . . . . . . . . . 45

3.1.2 Oligonucleotides . . . . . . . . . . . . . . . . . . . . . . . . 45

$3.1 .3 \quad$ Cell culture . . . . . . . . . . . . . . . . . . . . . . . . . . . . . . 45

3.2 Procedures and synthesis . . . . . . . . . . . . . . . . . . . . 46

3.2.1 $\quad$ Polymer/SWCNT dispersion . . . . . . . . . . . . . . . . . . . . . 46

3.2 .2 Peptide synthesis . . . . . . . . . . . . . . . . . . . . . . 46

3.2 .3 Conjugation of SMCC to DNA oligonucleotides . . . . . . . . . . . . 47

$3.2 .4 \quad$ ELISA Essay $\ldots \ldots \ldots \ldots$. . . . . . . . . . . . . . . . . . . . . 48

3.3 Analytical techniques . . . . . . . . . . . . . . . . . . . . . 48

3.3 .1 UV-Vis-nIR spectroscopy . . . . . . . . . . . . . . . . . . . 48 
3.3 .2 nIR spectroscopy and imaging $\ldots \ldots \ldots \ldots \ldots$

$3.3 .3 \quad$ Raman spectroscopy $\ldots \ldots \ldots \ldots$

$3.3 .4 \quad$ AFM spectroscopy $\ldots \ldots \ldots \ldots$

$\begin{array}{llr}4 & \text { Results and Discussions } & 50\end{array}$

4.1 Investigation of the interaction between redox active analytes and polmy-

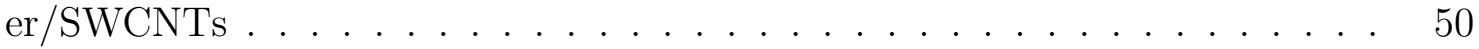

4.1 .1 Mechanisms for sensing in SWCNT-based sensors . . . . . . . . 50

4.1 .2 Impact of redox-active molecules on the fluorescence changes . . . . . 57

4.1 .3 Influence of reactive oxygen scavengers on fluorescence modulation. . 64

4.1 .4 Impact of polymer surface coverage on polymer/SWCNT response . . 65

4.1 .5 Influence of redox potential on SWCNT-sensor responses $\ldots \ldots \ldots$

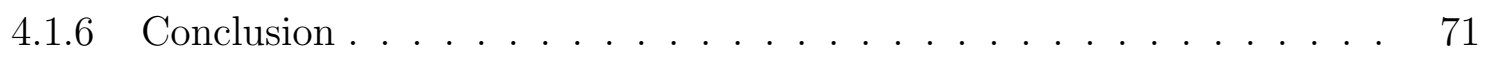

4.2 Development of sensors with tunable recognition units. . . . . . . . . . 74

4.2 .1 Modulation of the organic phase in SWCNT-based sensors . . . . . 75

4.2 .2 Modulation of integrin affinity by changing the RGD-motif . . . . . 78

4.2 .3 Effective affinity of a single RGD motif . . . . . . . . . . . . 81

4.2 .4 Cell adhesion on ssDNA-RGD/SWCNT hybrids . . . . . . . . 86

$4.2 .5 \quad$ Labeling of integrin on human blood platelets in the nIR . . . . . 88

4.2 .6 Conclusion $\ldots \ldots \ldots \ldots$

4.3 Egyptian Blue as novel nIR fluorophore . . . . . . . . . . . . . . . . . . . 91

4.3 .1 Investigation of Egyptian Blue nanosheets by atomic force microscopy

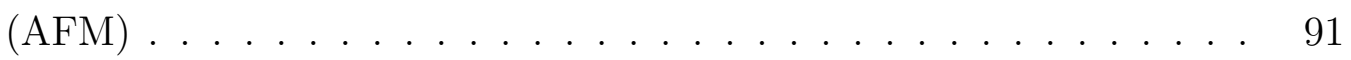

4.3 .2 Photophysical properties of Egyptian Blue nanosheets. . . . . . . . . 94

4.3 .3 Egyptian Blue as ratiometric sensor for dopamine detection . . . . . 100

4.3 .4 Conclusion $\ldots \ldots \ldots \ldots \ldots \ldots$

4.3 .5 Outlook for new nIR fluorophores . . . . . . . . . . . . . . 104

$\begin{array}{lll}5 \text { Conclusion and outlook } & 105\end{array}$

\begin{tabular}{lll}
\hline 6 & Appendix & 108
\end{tabular}

\begin{tabular}{lr}
\hline References & 134
\end{tabular}

\begin{tabular}{ll}
\hline Curriculum Vitae & 136
\end{tabular}

\begin{tabular}{lr}
\hline Danksagung & 141
\end{tabular} 


\section{Abstract}

Information transfer in the human brain is enabled by neurotransmitters - messenger molecules that diffuse between cells. Static concentration measurements are useful to identify molecules involved in neurotransmission, but so far dynamic measurements remain a challenge. However, detecting neurotransmitters with spatiotemporal resolution is essential to understand chemical neurotransmission and how it contributes to phenomena such as learning or degenerative diseases. This thesis investigates various strategies to develop nearinfrared (nIR) fluorescent sensors and the larger goal is spatiotemporal detection of small molecules such as neurotransmitters.

The first two sections of the thesis focus on functionalized single-walled carbon nanotubes (SWCNTs). SWCNTs can be visualized as a graphene monolayer rolled up into a hollow cylinder. They emit fluorescence in the range of $850-1700 \mathrm{~nm}$ (near infrared, nIR). The photophysics of SWCNTs is excitonic and upon excitation excitons move along the SWCNT axis. Even small changes in their local environment affect the fluorescence of SWCNTs, which makes them ideal building blocks for sensors. The exact photophysical sensing mechanism behind the fluorescence change of SWCNT-based sensors is not fully understood. To answer this question different possible sensing mechanisms were investigated by measuring how small redox-active molecules with different redox potentials, such as the neurotransmitter dopamine, ascorbic acid or cysteine, change the fluorescence of SWCNTs functionalized with different macromolecules, such as single stranded DNA (ssDNA) or lipids. First, a direct electron transfer between analytes and SWCNTs was ruled out. It was further verified that fluorescence changes are not due to scavenging of reactive oxygen species by analyte molecules or adsorption onto the free SWCNT surface. The results support a photophysical sensing mechanism based on the conformational change of the macromolecule around the SWCNT.

In the second part of the thesis, the recognition strategy was advanced by changing the functionalization concept. Despite high sensitivity of ssDNA/SWCNT sensors, their selectivity remains a challenge. Proteins and peptides are well-known for their specific molecular interactions in nature, but they have only rarely been used for SWCNT functionalization due to colloidal stability issues. Here, peptides were employed as recognition units in a modular sensor approach. As proof of principle this strategy was tested for a well-established cell surface receptor (integrin) ligand: Arg-Gly-Asp (RGD). The RGD peptide sequences were synthesized and conjugated to ssDNA in different geometries: the RGD recognition motif was either anchored between two ssDNA sequences (bridge) or attached to the ssDNA sequence at one side (linear). Next, the inhibition of $\alpha_{I I b} \beta_{3}$ integrin by different ssDNARGD/SWCNT hybrids was quantified by an enzyme-linked immunosorbent assay. The data 
suggest that all three parameters, such as ssDNA sequence, length, and geometry, modulate the affinity of the recognition unit (RGD) to its target (integrin). IC50 values for the different hybrids could be tuned from 20 to $309 \mathrm{nM}$. While SWCNTs serve as a confinement structure for the recognition unit, they also add a unique nIR fluorophore. This property was used to label $\alpha_{I I b} \beta_{3}$ integrins on human blood platelets in the nIR. These results show that it is possible to use a small peptide recognition motif and tune binding affinities by changing a DNA sequence, which could be highly interesting for the detection of other neurotransmitters such as glutamate.

In the last section another nIR fluorophore was explored: Egyptian Blue $\left(\mathrm{CaCuSi}_{4} \mathrm{O}_{10}, \lambda_{\text {em }}\right.$ $=930 \mathrm{~nm}$ ). Egyptian Blue is a calcium copper silicate with high quantum yield and a long fluorescent lifetime in the range of microseconds. First, Egyptian Blue was exfoliated into nanosheets and analyzed via atomic force microscopy. The nanosheets had a radius of approximately $36 \mathrm{~nm}$ and a thickness of $4-5 \mathrm{~nm}$. This height corresponds to 3 or 4 layers of Egyptian Blue unit cell layers. The mean fluorescence intensity of the nanosheets can be described by the product of height and radius of the particles, and thus correlates with the nanosheet volume. Therefore, the dimensionality of the nanosheets do not seem to affect the fluorescence quantum yield. In agreement with that, fluorescence lifetimes of the bulk material compared to nanosheets $(\tau \approx 124 \mu \mathrm{s})$ did not change. Finally, Egyptian Blue was successfully implemented as a nIR reference signal for ratiometric dopamine detection, which paves the way for applications of Egyptian Blue nanosheets in biomedical imaging. 


\section{Zusammenfassung}

Der Informationstransfer im menschlichen Gehirn erfolgt mithilfe von Neurotransmittern Botenstoffen welche an der Signalweiterleitung zwischen den Synapsen benachbarter Zellen beteiligt sind. Statische Konzentrationsbestimmungen helfen bei der Identifikation der Moleküle, die am Prozess der Signalübertragung beteiligt sind. Dynamische Messungen stellen allerdings nach wie vor eine Herausforderung dar. Dabei sind es gerade die dynamischen Prozesse der neuronalen Kommunikation, die für komplexe Prozesse wie Lernen oder Erkrankungen wichtig sind. Diese Arbeit untersucht Strategien zur Entwicklung von Sensoren, mit dem Ziel eine räumlich-zeitliche Konzentrationsverfolgung von kleinen Molekülen, wie den Neurotransmittern, zu ermöglichen.

Im Fokus der ersten beiden Abschnitte der Dissertation liegen Sensoren, die auf Kohlenstoffnanoröhren basieren (single-walled carbon nanotubes, SWCNTs). SWCNTs können als hohle Zylinder beschrieben werden, zusammengerollt aus einer Graphenmonolage. Sie fluoreszieren im Wellenlängenbereich von 850 - $1700 \mathrm{~nm}$ (nahes Infrarot, nIR). Die optoelektronischen Eigenschaften von Kohlenstoffnanoröhren können am besten mit Exzitonen beschrieben werden. Nach der Anregung bewegen sich die Exzitone entlang der Achse der Nanoröhre und erfassen selbst kleinste Veränderungen in der Nähe der Oberfläche der Kohlenstoffnanoröhren. Der genau photophysikalische Mechanismus der Fluoreszenzänderung durch kleine insbesondere redox-aktive Moleküle ist noch nicht vollständig bekannt. In dieser Arbeit wurden die exakten Gründe für die Fluoreszenzänderung erforscht, indem die Interaktion von funktionalisierten SWCNTs mit kleinen redoxaktiven Molekülen z.B. Dopamin, Ascorbinsäure oder Cystein quantifiziert wurde. Als organische Phase wurden Makromoleküle wie Desoxyribonukleinsäure (DNA) oder Lipide verwendet. In sich gegenseitig ergänzenden Experimenten konnten mehrere Mechanismen ausgeschlossen werden: (1) der direkte Elektronentransfer zwischen dem Analytmolekül und der SWCNT oder dem Makromolekül (2) eine Fluoreszenzänderung aufgrund der Reaktion des Analyts mit reaktiven Sauerstoffspezies und (3) eine Fluoreszenzänderung durch die direkte Adsorption der Analytmoleküle auf SWCNTs. Die Ergebnisse legen nahe, dass der Analyt Konformationsänderungen des Makromoleküls um die SWCNT bewirken kann, welche dann zu Fluoreszenzänderungen führen.

Im zweiten Teil der Dissertation wurden die Sensoren weiterentwickelt. SWCNTs mit nichtkovalenter Funktionalisierung mit DNA (DNA/SWCNTs) weisen Sensitivität bis hin zur nanomolaren Analytkonzentrationen auf. Um die Selektivität dieser Sensoren zu verbessern, wurden Peptide, die wohl-bekannt für die spezifischen Interaktionen sind, in die organische Phase um die Kohlenstoffnanoröhre inkorporiert. Um das Konzept zu testen, wurde die Peptidsequenz Arg-Gly-Asp (RGD) benutzt, die für ihre Affinität zu wichtigen Zelloberflächenrezeptoren (Integrinen) bekannt ist. Die Peptidsequenzen wurden synthetisiert und in un- 
terschiedlichen Geometrien an DNA-Sequenzen konjugiert. Das RGD-Motiv war entweder seitlich an einen DNA-Strang gebunden (lineare Geometrie) oder befand sich zwischen zwei flankierenden DNA-Strängen (Brückengeometrie). Anschließend wurde die Bindungsaffinität von verschiedenen DNA-Peptid/SWCNT Komplexen zum humanen $\alpha_{I I b} \beta_{3}$ Integrin quantifiziert. Die Resultate zeigen, dass sowohl DNA-Länge, als auch Sequenz und Geometrie die Bindungsaffinität von RGD-Motiv zu Integrin beeinflussen. Die IC50 Werte für unterschiedliche DNA-Peptid/SWCNT Komplex konnten so von 20 bis $309 \mathrm{nM}$ variiert werden ohne eine Veränderung des RGD Motifs. Die Kohlenstoffnanoröhren dienten hier nicht nur als Gerüst für die DNA-Peptid Makromoleküle sondern auch als nIR Fluorophor und wurden in dieser Eigenschaft in weiterführenden Zellexperimenten eingesetzt. Beispielsweise konnten so $\alpha_{I I b} \beta_{3}$ Integrine auf der Oberfläche von Thrombozyten im nIR markiert werden. Dieser Ansatz zeigt, dass das Immobilisieren eines kleinen Peptid-Motivs möglich ist und könnte in der Zukunft die Detektion von Neuortransmittern wie Glutamat möglich machen.

Im letzten Kapitel wurde Ägyptisch Blau untersucht $\left(\mathrm{CaCuSi}_{4} \mathrm{O}_{10}, \lambda_{e m}=930 \mathrm{~nm}\right)$, ein anorganisches Mineralpigment aus der Klasse der Cuprorivaite. Dieses Kupfersilikat ist, ähnlich wie die Kohlenstoffnanoröhren, ein nIR Emitter. Zuerst wurde das Ägyptisch Blau Pulver exfoliert und mittels Rasterkraftmikroskopie untersucht. Die hergestellten 2D Nanoblätter wiesen einen Radius von ca. $36 \mathrm{~nm}$ und eine Höhe von $4-5 \mathrm{~nm}$ auf. Diese Höhe entspricht 3 oder 4 Schichten von einzelnen Ägyptisch Blau Einheitszellen. Trotz der Verkleinerung auf quasi 2D Nanoblätter behielt Ägyptisch Blau seine nIR Fluoreszenz und die Fluoreszenzlebensdauer von $\tau \approx 124 \mu$ s änderte sich nicht. Die mittlere Intensität der 2D Nanoblätter ließ sich durch das Produkt aus Höhe und Radius der Partikel beschreiben. Damit korrelierte die Fluoreszenz eines Ägyptisch Blau Partikels mit seinem Volumen und es konnten keine darüber hinaus gehenden Einflüsse der Dimensionalität gefunden werden. Anschließend wurde Ägyptisch Blau erfolgreich als (ratiometrisches) nIR Referenzsignal für Kohlenstoffnanoröhren-basierte Dopamin Sensoren eingesetzt. Damit könnten 2D Nanoblätter aus Ägyptisch Blau eine neue Klasse von nIR Fluorophoren für bildgebende biomedizinischen Anwendungen darstellen. 


\section{Introduction}

\subsection{Chemical signaling in the brain}

The human nervous system is composed of about 86 billion neuronal cells (neurons) [1]. For a sufficiently fast functioning of the brain, highly efficient communication pathways among large numbers of cells are most essential. The cell-cell communication in the brain, also called neurotransmission, is carried out through chemical messengers (neurotransmitters) and electrical impulses [2]. Electrophysiological signaling between neurons is an integral part of the brain function [3]. However, the emphasis of this thesis lies on the other component of the neuronal communication process: the chemical signaling. This work will focus on developing near-infrared sensors for spatiotemporal detection of small molecules, e.g. neurotransmitters. Such sensors are essential to understand the processes underlying neuronal cell-cell communication and how neurotransmitters contribute to larger phenomena such as the learning process or degenerative diseases of the central nervous system (CNS).

To identify the requirements to study the neurotransmitter release, we have to contemplate the geometry of the process. Neuronal cells form elongated protrusions (axons and dendrites), which come together at conjunction points - synapses [3]. Neurotransmitters are stored in vesicles inside of axon terminals. Electrical potential at the axon causes the vesicle to fuse with the cell membrane and release neurotransmitters (a process called exocytosis) [4]. Released neurotransmitters cross the synaptic cleft $(\approx 20 \mathrm{~nm})$ and bind to specialized receptors on the target cell, which can trigger further electrical or chemical cascades [5]. A typical picture of a neurotransmitter release is shown in Fig. 1.

a

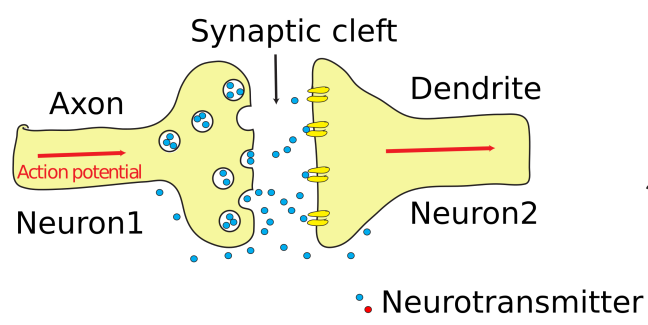

b Chemical transmission

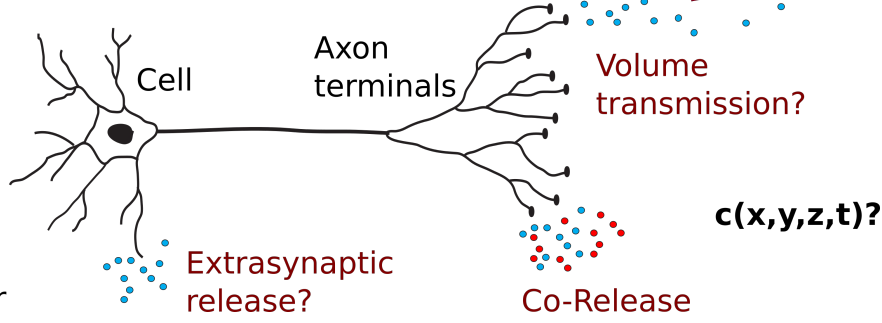

Figure 1: Neurotransmitter release processes. (a) Schematic of a chemical synapse consisting of an axon terminal and a dendrite, (b) Possibilities of neurotransmitter release processes and diffusion pathways. Adapted from own work 6 .

While this picture is useful to get a comprehensive overview of a highly complex matter, the 
simplification reduces the role of neurotransmitters to a simple signal transmission between two sides of a synaptic cleft. In reality, a part of the released molecules can diffuse through intracellular fluids to a far-off neuron and only then bind to a distant receptor (volume transmission) [7], [8]. To further complicate the matter, more than one neurotransmitter can be released at a time (co-release), or neurons can suddenly switch and release different kind of neurotransmitters (switching) [9], [10]. These dynamic facets of chemical communication can only be ascertained with high spatial and temporal resolution detectors. Static neurotransmitter concentrations in brain tissues are easily obtained via mass spectroscopy (MS) or high-performance liquid chromatography (HPLC) [3]. Even though such analytical methods can characterize the chemicals involved in neurotransmission, the dynamic aspect is lost and with it the possible comprehension of neurotransmitter contribution to larger and more complex phenomena.

\subsubsection{Requirements for spatiotemporal neurotransmitter sensors}

In the following section, we will review time, size, and concentration scales that are relevant for neurotransmitter release processes. Even though the exact numbers depend on the model organism, to get an idea of the scales and ranges we can focus on a single cell line and a specific neurotransmitter; in our case pheochromocytoma cells (PC12) and dopamine.

There are several molecule types involved in neuronal communication. Aside from neuropeptides, one can distinguish 4 classes of chemical neurotransmitters: 1) biogenic amines (e.g., dopamine, epinephrine, and serotonin), 2) amino acids (e.g., $\gamma$-aminobutyric acid (GABA), L-glutamic acid), 3) acetylcholine and as well a less defined group of 4) 'unconventional' neurotransmitters that do not fulfill classic neurotransmitter criteria, such as purines (e.g., adenosine triphosphate) or gaseous neurotransmitters (nitric oxide $(\mathrm{NO})$, carbon monoxide $(\mathrm{CO})$ ) [1]. Catecholamine neurotransmitters are an important under-group of the biogenic amines and include molecules composed of a catechol unit (benzene with two hydroxyl side groups) and an amine side-chain 12. Fig 2 provides chemical structures of discussed catecholamines and other neurotransmitters. In the first segment of this work, I will focus mainly on catecholamines and especially on dopamine (3,4-dihydroxyphenethylamine). Apart from the brain, dopamine can also be locally synthesized in peripheral systems (pancreas, kidneys, vascular system, etc.) and affects cells in close proximity to the release point [13]. It was only recently discovered that immune cells, such as T-cells, can both produce and detect neurotransmitters [14. Within the central nervous system (CNS) dopamine circulation plays a major role in reward-motivated behavior, associative learning, and drug addiction [15]. The most prominent dopaminerelated dysfunction is associated with Parkinson's disease [16]. On a more complex scale, 
there are also indications of its involvement in schizophrenia, bipolar disorder and attention deficit hyperactivity disorder (ADHD) [17].

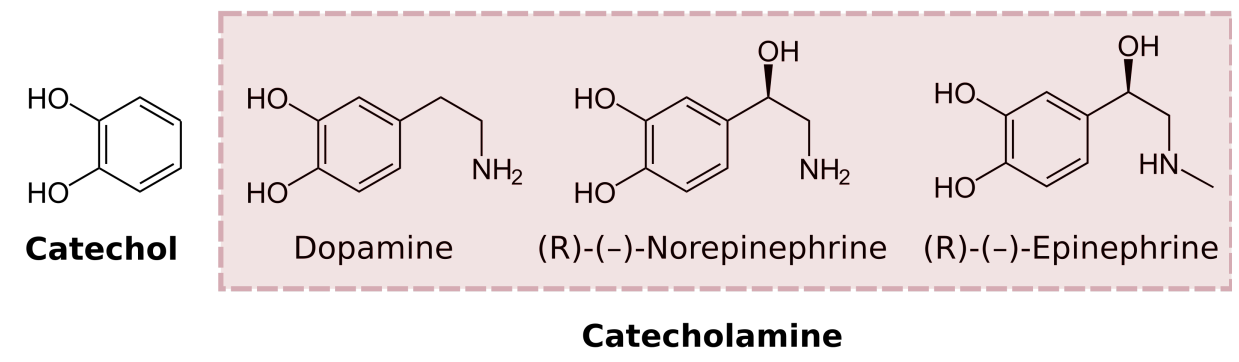

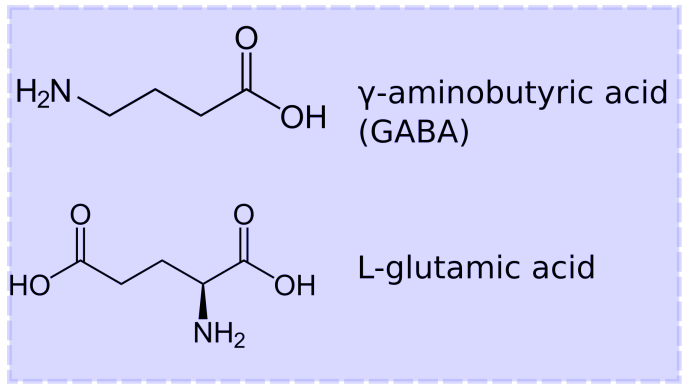

Amino acids

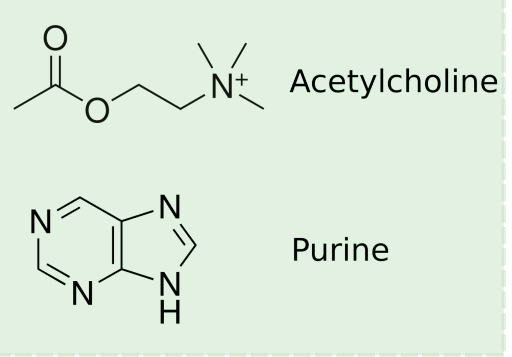

Acetylcholine and purine

Figure 2: Examples of neurotransmitters mentioned in this section. Chemical structures of (1) chatechol unit and catecholamine neurotransmitters (dopamine, norepinephrine and epinephrine), (2) amino acids ( $\gamma$-aminobutyric acid (GABA), L-glutamic acid), (3) acetylcholine as a standalone group and purine as a representative of non-conventional neurotransmitters.

The geometry of the neurotransmitter release system dictates criteria for the trajectory of neurotransmitter molecules after the release time-point. A well-studied model system of pheochromocytoma cells (PC12) will provide us with starting numbers to estimate the target concentrations and temporal limitations [18]. Upon stimulation PC12 cells release catecholamines, mostly dopamine, which can be detected by electrochemical techniques, such as amperometry (more about this and other electrochemical detection techniques in section 1.2). Electrochemical techniques, dynamic light scattering (DLS) and transmission electron microscopy (TEM) suggest temporal and spatial dimensions of neurotransmission process to lie in ms- and nm-range [19]. A released neurotransmitter travels with the diffusion coefficient $D \approx 0.3-1 \mu \mathrm{m}^{2}$ per ms, a receptor with $D \approx 10^{-2} \mu \mathrm{m}^{2}$ per s [20], [21]. These diffusion velocities suggest that a receptor would cover $6 \mathrm{~nm}$ in $1 \mathrm{~ms}$ and a neurotransmitter molecule $20 \mathrm{~nm}$ in $0.07-0.2 \mu \mathrm{s}$, which indicates a highly dynamic process [19]. Therefore, it would not be reasonable to focus exclusively on absolute concentrations since they will change dramatically over time and distance. Concentration profile of a molecule diffusing 
from a release point can be formulated as:

$$
c(r, t)=\frac{\frac{-r^{2}}{e^{4 d t}}}{(D t)^{\frac{3}{2}}},
$$

with $D$ as the diffusion constant and $c$ the concentration at distance $r$ and after time $t$ [7]. Fig. $3 \mathrm{~b}$ shows the time-dependent profile of a neurotransmitter from the release point. The plot indicates that not only sensor sensitivity is crucial for detection but also a sufficient acquisition speed.

Another aspect to consider is not only the binding of the analyte to the sensor but also a sufficiently fast reversibility of the sensor response to allow for temporal resolution of dynamic processes. Obviously, it would be a misguided strategy to create highly sensitive yet non-reversible sensors, as this can be already achieved by highly efficient stationary techniques (HPLC, MS, etc.). In a sensor array to monitor dopamine diffusion, the specific kinetics parameters (rate constants, $k_{o n}$ and $k_{o f f}$ ) of the sensors would be most important parameters. Rate constants determine how long it takes until an analyte will bind and how long a sensor will stay saturated after binding. Sensors with a low dissociation constant $\left(K_{d}=\frac{k_{o f f}}{k_{o n}}\right)$ will saturate almost immediately and lose their sensing ability for a long period of time. It is therefore favorable to have only a short binding period to resolve not only the concentration profiles but also the subsequent molecule diffusion to further locations. So far, Monte Carlo simulations performed by Daniel Meyer and Annika Hagemann demonstrated that rate constants of $k_{o n}=10^{6} \mathrm{M}^{-1} \mathrm{~s}^{-1}$ and $k_{\text {off }}=100 \mathrm{~s}^{-1}$ cause sensor response images similar to the concentration profiles of dopamine release 22]. 

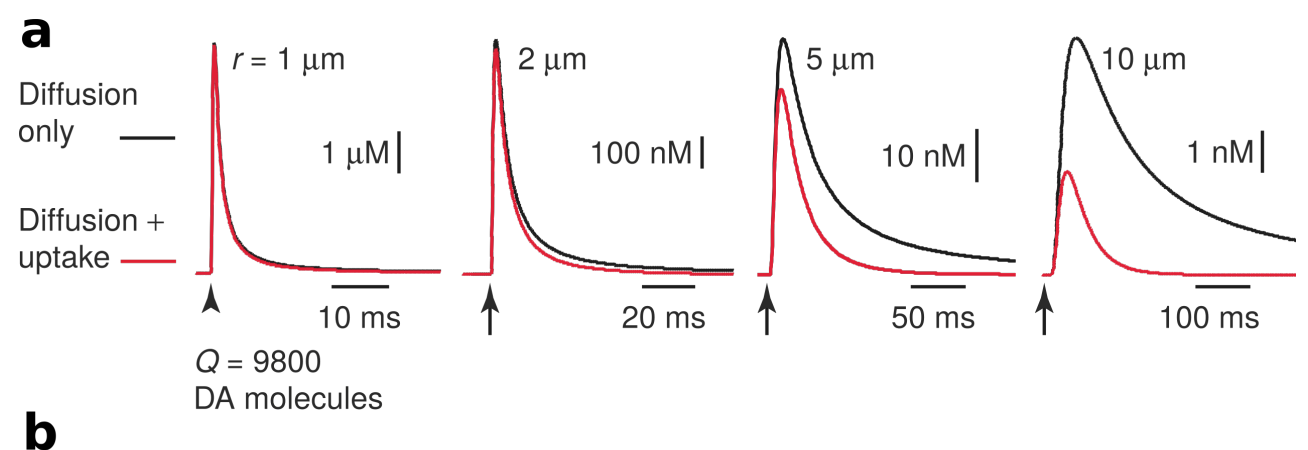

b

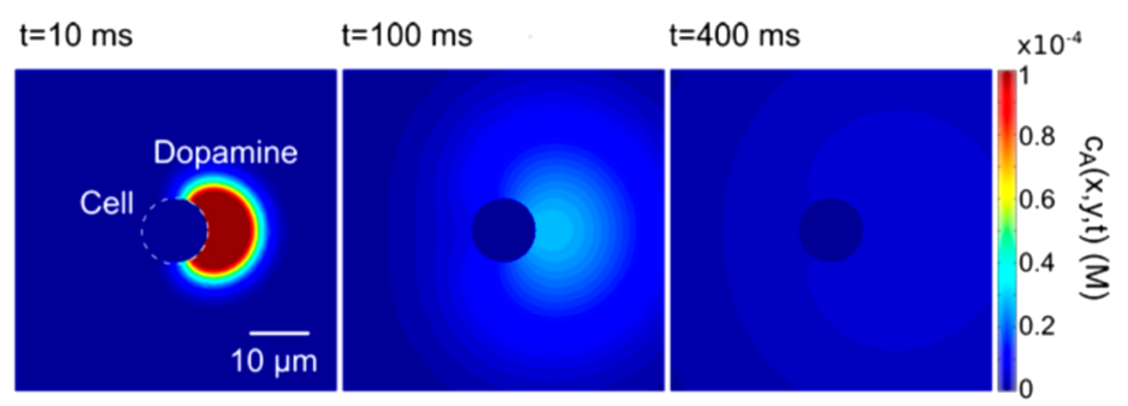

Figure 3: Diffusion profiles of neurotransmitter concentration after release. (a) Simulated concentrations of dopamine release from a release point in radial distances, without uptake by receptors (black) and with uptake (red). Adapted and modified from [7], (b) Diffusion of dopamine after exocytosis from a single vesicle on the example of a single cell $(\mathrm{d}=10 \mu \mathrm{m})$. Adapted from $[22]$.

To conclude the aforementioned limitations, following specifications for neurotransmitter sensors would be desirable to cover the spatiotemporal dynamics: (1) ms-fast nanoscaled sensor with nM-sensitivity (concentration gradient around the cell due to movement of neurotransmitters) and (2) the dynamic range of $\mu \mathrm{M}-\mathrm{mM}$, as high concentration will be observed for immediately after the release (concentration of $\mathrm{mM} \approx 1 \mathrm{~ms}, \mu \mathrm{M} \approx 100 \mathrm{~ms}$ ), (3) the binding kinetics of the analyte to the sensor lies in the ms range to detect the neurotransmitter before it has diffused away and at the same time, (4) reversible binding to monitor the temporal profiles of dynamic processes. Finally, the size of the sensors should be on the nanoscale range and, preferably, the detection itself should be carried out non-invasive. With that in mind, in the next section we can take a look at the current neurotransmitter sensor approaches and see which points are already covered and where there is still a high demand for suitable sensors. 


\subsection{Techniques to determine local neurotransmitter concentrations}

Static concentration measurements have helped to identify many molecules involved in neurotransmission, but so far dynamic measurements remain a challenge for the analytical community. There are currently two approaches for local neurotransmission detection: (1) optical sensing, mostly based on fluorescent building blocks, which reveals spatial information and (2) electrochemical sensing that can achieve superior time resolution.

\subsubsection{Electrochemical sensing}

Most of the classical neurotransmitters have been identified via electrochemical methods by the start of the 1970s and further specified with the help of high-pressure liquid chromatography (HPLC) or combined gas chromatography/mass spectrometry (GC/MS) [23], 24], 25], [26], 27]. In 1973, Ralph Adams was the first to apply a traditional voltammetric technique to detect oxidizable biogenic amines in the brain of an anesthetized rat 28. Nowadays, we have to consider the possibility of interference with ascorbic acid that is also present in the brain and has a similar redox potential to some neurotransmitters [29]. Still, current biological studies would not be possible without the quantitative information about neurotransmitter concentrations provided by the electrochemical methods [14], [30]. So far, there are two major electrochemical neurotransmitter detection techniques: (1) amperometry and (2) cyclic voltammetry (CV) 31, 32.

Amperometric detection is based on a voltage (potential) applied between two electrodes and its sensitivity to incoming redox-active molecules. Any electroactive analyte will be either oxidized or reduced, and the current measured [33. In 1990, Wightman et al. could directly monitor time-resolved exocytotic events on the millisecond timescale [34. To detect vesicle fusion an electrode is held to a positive potential and brought in close proximity to the cell. As soon as the vesicle fuse and release neurotransmitters into the solution, redox active neurotransmitters get oxidized at the electrode tip. Size and sequence of following current spikes provide information about the number of vesicles and the release probability [28], 35].

First experiments involved only single electrodes, thus providing no spatial information. To improve it, recent electrochemical techniques aim to implement multiple and smaller electrodes. Increasing electrode density can be used to raise both the number of data points for samples with multiple cells or to allow a more accurate imaging of a single cell. Lindau et al. expanded the method to use an electrochemical detector (ECD) array with 4 platinum microelectrodes around the cell (Fig. 4a) [36]. Then chromaffin cells are seeded onto the ECD array and chemically stimulated into catecholamine release. Observed oxidation current reproduces the direction and volume of the neurotransmitter that reach the electrode tip. 
Thus, it was possible to achieve electrochemical imaging of fusion pore openings and estimate the most probable exocytosis site on the cell surface. One has to keep in mind possible interference due to introducing an ECD array into the cells and using a patch pipette. Such handling can disturb the normal behavior of the cell and the molecule diffusion pathways, and interfere with an in vivo application.
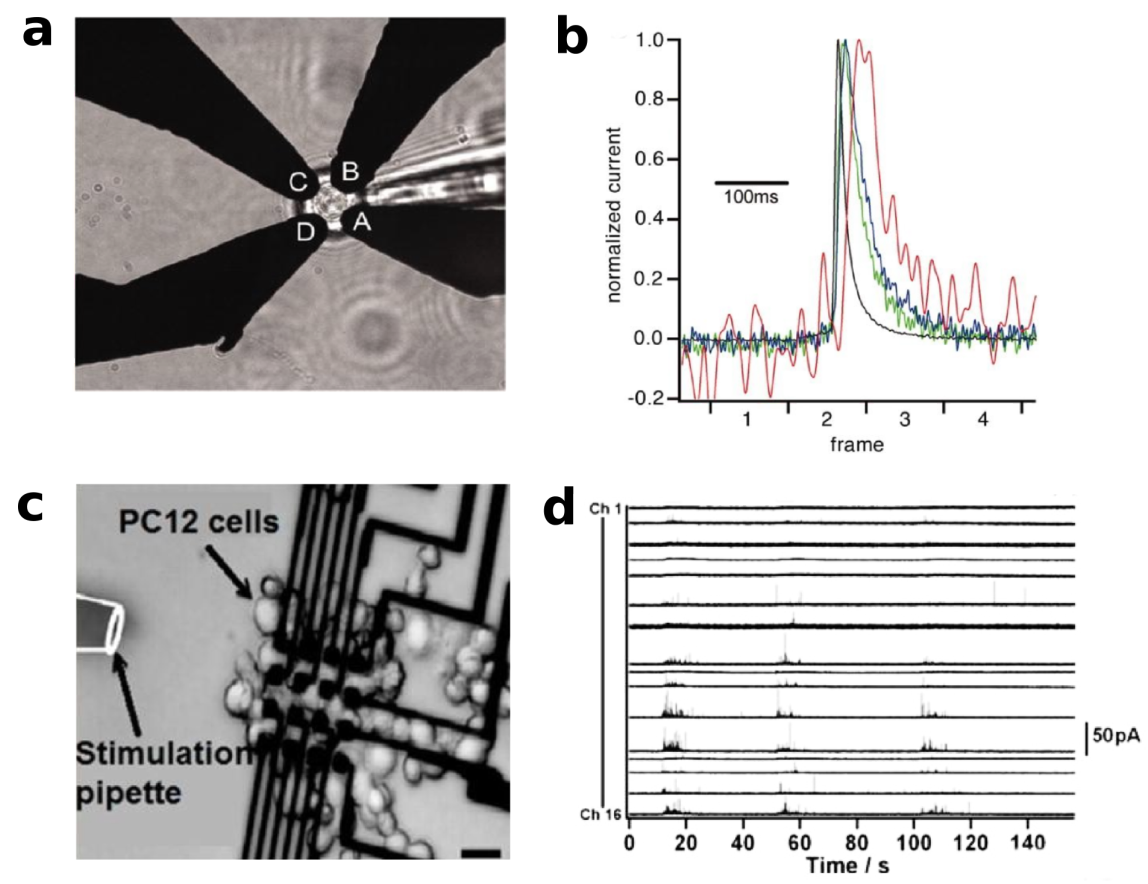

Figure 4: Electrochemical dopamine detection. (a) Image of a chromaffin cell upon an electrochemical detector (ECD) array with four electrodes (A-D), (b) Currents from the four electrodes (A-D, as black, blue, green, and red line) reveal spatiotemporal differences, (c) Image of a setup with the 16-electrode ensemble over a cluster of PC12 cells and the stimulation pipet. (d) Amperometric traces from different electrodes shown in (c) after cell stimulation. Adapted from [6].

A different microelectrode array (MEA) with a more bio-compatible material was designed by Ewing et al. 37. Here, 16 electrodes were integrated into a PDMS chamber coated with collagen. PC12 cells were cultured on top of the MEAs device and the single electrodes provided individual readout. Fig. $4 \mathrm{f}$ shows traces from different electrodes. Due to this arrangement, it was possible to record exocytotic events and dopamine release down to a spatial resolution of $2 \mu \mathrm{m}$. This resolution is useful for collecting information about small cell clusters (e.g. to see how pharmacological treatment could affect dopamine release) but cannot provide any information about the heterogeneity of dopamine release within one cell. To achieve this resolution the electrodes were minimized even more, until in 2014, Ewing et al. increased the number of electrodes to 36 and achieved subcellular resolution 38. 
Cyclic voltammetry (CV) has a slightly different approach. During CV one sweeps the potential of the working electrode at a specific sweep rate (in volts/second). The output results in a curve of measured current vs. time. After the reduction potential of the analyte is reached and no more reducible analyte is available, the reduced analyte starts getting re-oxidized. Thus completing the circle and accounting for the term of cyclic voltammetry.

Fast cyclic voltammetry (FSCV) is a class of cyclic voltammetry with a high scan rate (up to $1 \times 10^{6} \mathrm{~V} \cdot \mathrm{s}^{-1} \approx 10 \mathrm{~Hz}$ ) which allows for high temporal resolution [39]. FSCV is a frequently used method for detection of neurotransmitters in biological systems [40]. Here, the neurotransmitters are repeatedly oxidized and reduced resulting in a current of electrons on a nanoampere scale. With a known time scale of the voltage oscillation, concentration change of dopamine can be calculated down to $1 \mathrm{nM}$ in real time [41]. The typical size of electrodes for FSCV lies in the order of micrometers, though there are single reports about nanoscale electrodes for dopamine detection [42], [43].

In conclusion: over the last decades, classical electrochemical methods have provided neurochemists with important insights into the chemical nature of neurotransmitters, the number of released molecules, and the time resolutions of the processes involved. A clear advantage of electrochemical techniques is their high time resolution $(\approx 100 \mu \mathrm{s})$ and the possibility of integration in various designs [44]. As for its drawbacks, there are several:

- Diffusion of released neurotransmitters could be affected by the electrodes implemented into the system, which would require additional calculations to reconstruct the original concentration profiles.

- CV is dependent upon different reduction potentials of analytes. CV can distinguish between molecules with different redox potentials but cannot discriminate compounds with similar potentials, however different their chemical structures might be.

- Another limitation of CV comes from the cross-talk between individual electrodes 42, 43 .

- The analytes have to be redox-active which limits the number of neurotransmitters. Dopamine or serotonin can be detected while other important neurotransmitters, such as L-glutamate or $\gamma$-aminobutyric acid (GABA), can not.

- Low spatial resolution. Despite current progress in miniaturization of electrodes, most structures are still too big in comparison to a neurotransmitter release site [44]. 


\subsubsection{Optical sensing}

While the strength of electrochemical sensing lies in its high temporal resolution and the major drawback in the poor spatial resolution, the situation for optical sensing is reversed. Optical sensing can be achieved by: (1) using fluorescent materials or molecules as building blocks for sensors, or (2) implementing genetically encoded fluorescent sensors into biological structures involved in neurotransmitter release.

One of the first examples of dopamine detection with a fluorescent nanoparticle was reported by Willner et al. [45]. They functionalized Cd-Se-ZnS quantum dots (QD) with a boronic acid derivate. This moiety is known for its ability to bind molecules with two adjacent hydroxy-groups. Thus, this principle can not only be applied to detection of catecholamines but also of sugars (e.g point-of-care glucose detectors for diabetes). In the first step, an organic fluorophore is conjugated to dopamine. Upon binding of dopamine-fluorophore conjugate to the boronic acid moiety, there is a Förster resonance energy transfer (FRET) to the fluorophore and the fluorescence of the QD is decreased. When an unbound dopamine molecule from the solution replaces its dopamine-fluorophore counterpart on the $\mathrm{QD}$, the fluorescence of the sensor is restored. An important disadvantage of this technique is the lack of reversibly in a biological setting. Once all dopamine-fluorophore conjugates are replaced on the GO, the sensor is saturated and cease to detect further molecules. Another nanomaterial used for dopamine detection is graphene oxide (GO). Emission of GO nanosheets is highly sensitive to binding of other molecules and is quenched by dopamine, probably via $\pi-\pi$ stacking and photo-induced charge transfer [46]. This reaction allows to measure dopamine concentrations in urine samples down to $2.3 \mu \mathrm{M}$, but the selectivity of such sensors is not sufficient to distinguish between various catecholamines or other small molecules with extended $\pi$-systems 47 .

A reasonable approach to increase selectivity is to mimic existing molecular recognition units. Nature has already developed several receptors for such an important molecule class as neurotransmitters and one can integrate those natural neurotransmitter receptors into artificial sensors [48]. One of the first examples for such composition resulted in a genetically encoded fluorescent sensor for glutamate with $K_{d}$ of $630 \mathrm{nM}$ [49]. The sensor is a complex of a bacterial glutamate-binding protein (ybeJ) and two fluorescent proteins (YFP and CFP). Conformational changes of the glutamate binding protein induce FRET and can be optically detected. By attaching a periplasmic binding sequence to the sensor, the sensor is connected to the membrane of the transfected cells. Docked on the surface of the cells the sensors can determine glutamate after electrical stimulation of hippocampal neurons. The novelty of this approach lies in the possibility to image the evolved neurotransmitter themselves and not only the related processes such as vesicle fusion. Despite the groundbreaking approach this technique still has several limitations: 1) both absorption and emission show width 
spectra and make measuring an exact change difficult, 2) the conformational change of the binding protein is small, which results in a poor signal to noise ratio, 3) low photostability of fluorescent proteins (compared with more stable organic dyes or fluorescent nanomaterials without any bleaching at all) limits the applicability of the system, 4) conformational change upon binding of the neurotransmitter and its impact on the FRET signal is hard to foresee and tune beforehand. That makes a rational design of such sensors difficult.

To overcome some of the objections Johnsson et al. presented a semisynthetic approach. Their biosensor is a complex of a self-labeling protein tag (SNAP-tag), an organic fluorophore and a metabolite-binding protein [50]. SNAP-tag is conjugated to a synthetic ligand (L) of the binding protein and a second fluorophor. In the absence of the analyte, the synthetic ligand $(\mathrm{L})$ is bound to the metabolite-binding protein and the sensor protein is in closed conformation. Close proximity of both donor and acceptor fluorophores result in a high FRET efficiency. When the analyte is introduced into the system, it displaces the ligand from the binding protein. The sensor protein shifts from a closed to an open conformation. This change can be measured by a ratiometric change in the fluorescence intensities of the two fluorophores. So far, this technique has been leading in the neurotransmitter dynamics research. Its modular approach and an existing library of available SNAP-tags and fluorophores allow adaptation to new biological systems. A drawback of this method is the need for cell transfection and manipulation. This can affect complex biological experiments and make in vivo measurements impossible.

A further development of this technique allowed to genetically encode the protein part of the sensor and produce it on the cell surface [50]. This development lead to a new acronym SNIFIT (SNAP-tag based indicator proteins with a fluorescent intramolecular tether) and allowed to measure the metabolite concentrations on the cell surface, as well as indirect measurements of glutamate, acetylcholine and $\gamma$-aminobutyric acid (GABA) concentrations [51], 52], 53]. Figure 7illustrates this principle by showing a SNIFIT sensor for acetylcholine (ACh-SNIFIT). 

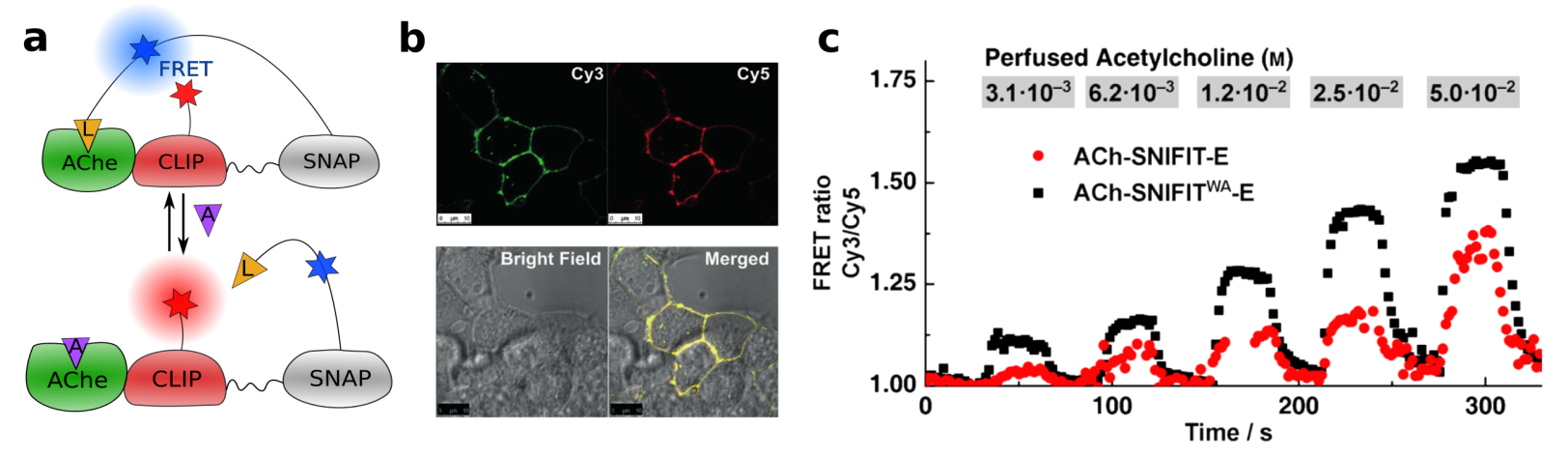

Figure 5: Schematic of a semisynthetic SNIFIT based sensor for acetylcholine (AChSNIFIT). (a) A ACh-SNIFIT sensor contains: (i) acetylcholinesterase (AChE, green), (ii) a labeling protein tag (CLIP) used to introduce a FRET donor (Cy5, blue star), (iii) a self-labeling protein tag (SNAP) with a FRET acceptor (Cy3, red star), and (iv) a synthetic ligand for $\mathrm{AChE}$ (L, yellow triangle). When the analyte (ACh, violet) displaces the ligand (L), FRET efficiency changes. (b) Confocal images of HEK cells expressing ACh-SNIFIT, labeled with Cy3 and Cy5. Scale bars: $10 \mu \mathrm{m}$, (c) Titration of ACh-SNIFIT induced cells with various ACh concentrations, shown as the ratio of donor $(\mathrm{Cy} 5)$ to acceptor $(\mathrm{Cy} 5)$ emission. Adapted with permission from 52

As the metabolite-binding protein they use acetylcholinesterase (AChE) that hydrolyzes acetylcholine to choline. Cy5 and Cy3 serve as the FRET donor and acceptor, respectively. This sensor has been anchored to the outer membrane of living cells and enable ACh detection (Fig. 77). While this technique is currently leading in neurotransmitter release imaging, it has major drawbacks: (1) the opening and closing kinetics of the sensor protein are several orders of magnitude slower than the neurotransmitter release kinetics (seconds vs. milliseconds), (2) the sensitivity of the sensors lies in the $\mathrm{mM}$ range. Combined those two specifics prevent detection of fast diffusing molecules. 


\subsubsection{Optical sensing based on single-walled carbon nanotubes (SWCNTs)}

There is still one sensor strategy that was so far omitted from the list of neurotransmitter detection techniques. In the next chapter we will go into detail on its mechanism and detection limits. The technique is based on single-walled carbon nanotubes (SWCNTs). SWCNTs non-covalently functionalized with single-stranded deoxyribonucleic acid (ssDNA) can detect dopamine concentration via emission increase [54]. Specifically, normalized nIR fluorescence increase can go up to $200 \%$ upon addition of $100 \mu \mathrm{M}$ dopamine [55]. After the stimulation of PC12 into catecholamine release, ssDNA/SWCNTs revealed spatiotemporal diffusion profiles of neurotransmitters and allowed to calculate the release points on the cell surface [56]. Sensors based on functionalized SWCNTs provide: 1) binding kinetics in the sufficient time regime (ms-range) to trail temporal concentration profiles, 2) small dimensions, enough to be implemented into a sensor array and provide spatial information of the release sites $(<500 \mathrm{~nm})$, and 3) analyte sensitivity down to the single-molecule level (100 pM) for individual nanosensors [57], [58], [59]. SWCNTs provide high photostability and tissue transparency in their emission range [60], 61]. In the following sections we will further discuss this sensor type, with focus on their geometry and structure 2.1.2, optical and electrical properties (2.1.3), and a detailed discussion of their advantages and disadvantages as neurotransmitter bionanosensors in 2.2.3. Afterwords, we will introduce the concept of functionalization of SWCNTs, and discuss how it affects the sensor selectivity 4.2. At the end of the section, we will briefly raise the subject of another nIR fluorophore that can be added to the library of the nIR nanomaterials for sensor applications (2.3.3). 


\section{Theoretical background}

\subsection{Single-walled carbon nanotubes (SWCNTs)}

As mentioned in the previous section, SWCNTs are new and versatile building blocks for neurotransmitter detection [54], [58], [62]. Due to their unique photophysical properties and the $1 \mathrm{D}$ geometry (that makes them sensitive to smallest changes in their environment) SWCNTs are one of the most attractive nanomaterials for a variety of electrochemical sensing, biosensing and bioimaging applications [59], [63], [64], [65], 66]. The following section will cover structure and synthesis of SWCNTs, as well as the chemical and photophysical properties resulting from different structures. The next section will introduce various strategies for functionalization of SWCNTs, which is the crucial step in SWCNT-based sensor design.

\subsubsection{Synthesis of SWCNTs}

Single-walled carbon nanotubes (SWCNTs) can be visualized as a graphene monolayer rolled up into a hollow cylinder. As the name suggests, multi-walled carbon nanotubes (MWCNTs) consist of multiple layers of graphene stacked into each other. The image of stacking and rolling graphene is beneficial to visualize carbon nanotube geometry, but the real synthesis process differs greatly from the simplified picture.

The easiest way to produce CNTs is to light a candle. The quantities of produced CNTs during oxidation processes are almost negligible and mixed in with other forms of carbon that are produced in abundance. Therefore, the focus of carbon nanotube synthesis lies in producing a useful quantity of pure nanotubes with minimal contamination and definite structure. Iijima is widely acknowledged for his discovery of MWCNTs in 1991, during evaporation of carbon in an argon-filled vessel - an approach used mostly for the production of fullerene $\mathrm{C}_{60}$ [67]. The interest in a new compound was awakened and two years later the lab of Iijima successfully introduced single-walled nanotubes into the scientific society [68]. However, it is far less known that in 1952, Radushkevich and Lukyanovich reported their discovery of 'worm-like' structures that were observed during the decomposition of carbon monoxide $(\mathrm{CO})$ on iron particles at $600^{\circ} \mathrm{C}$ [69]. Based on the TEM images, the researchers concluded that the formed product must be elongated filaments or 'needle-like' carbon structures with diameters in the nanometer range [70]. As such, first discovery of CNTs was not appreciated due to the lack of awareness of possibilities in the field of nanotechnology and the lack of proper techniques to further investigate the curious structures 71 . 
a

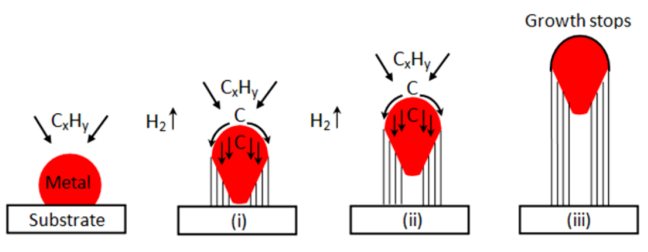

b

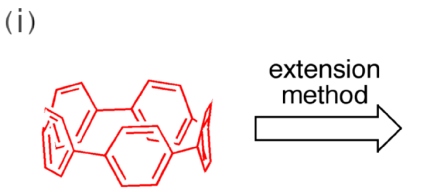

[5]-cycloparaphenylene

(ii)
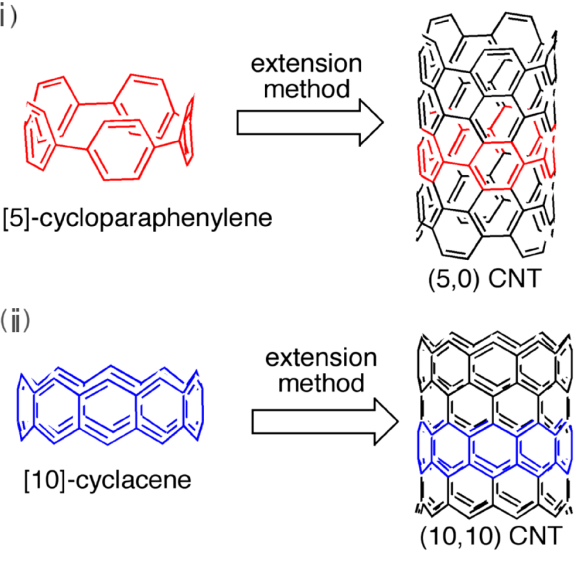

$(5,0)$ CNT

$(10,10)$ CNT

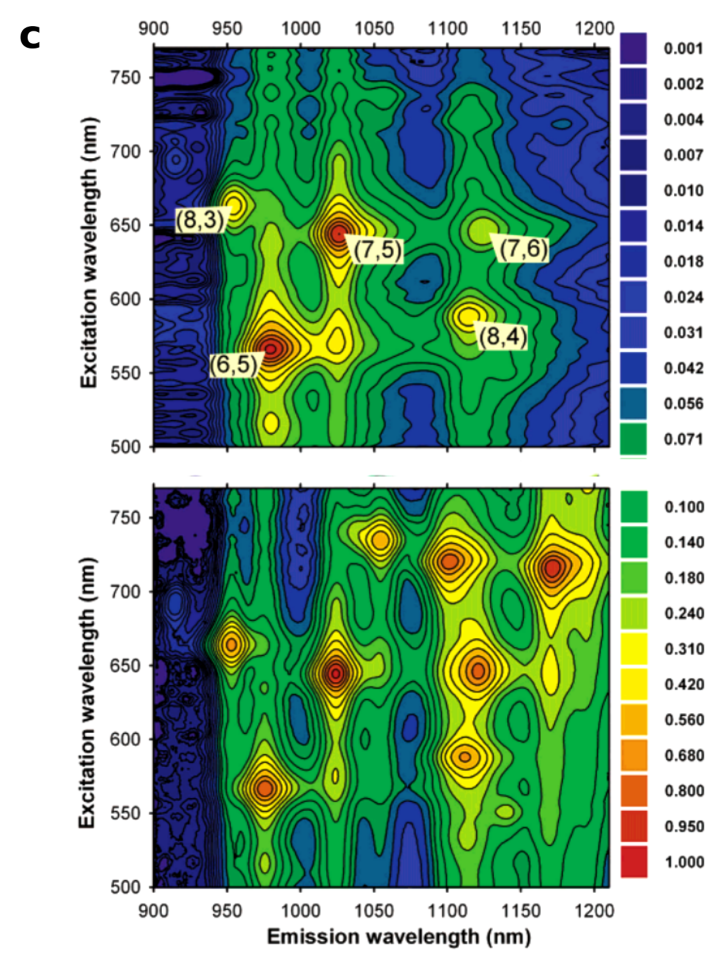

Figure 6: Various synthesis strategies for CNTs. (a) Model mechanisms for tip-growth of CNTs, (b) Novel bottom-up syntheses of SWCNTs based on ring-shaped carbon macrocycles as templates for growing CNTs, and (c) Contour plots of normalized fluorescence intensities for the CoMoCAT sample (top frame) and the HiPCO sample (bottom frame). Adapted with permission from (a) [72], (b) [73], and (c) [74].

The classical synthesis techniques are: (1) carbon arc-discharge, (2) laser ablation and (3) chemical vapor deposition (CVD) 61]. CVD can be further divided into either a process involving a gaseous catalyst (high-pressure carbon monoxide decomposition, HiPCO), or a supported catalyst (Cobalt and Molybdenum catalyst process, CoMoCAT) [74]. Most of these processes require addition of metal catalysts (such as Ni, Co, Fe, etc.) [75]. While high temperature techniques (arc discharge or laser ablation, $>1000^{\circ} \mathrm{C}$ ) were the first used to produce CNTs and are still leading in producing of large quantities, it allows little control over purity and homogeneity of the product. Low temperature CVD techniques $\left(\approx 800^{\circ} \mathrm{C}\right)$, working at atmospheric pressures, execute better control over orientation and alignment of CNTs [76]. Here, a mixture of a hydrocarbon gas (e.g. ethylene or methane) and a process gas (e.g. ammonia, nitrogen, etc.) react on a heated metal substrate. Hydrocarbon gas decomposes, deposits on the metal substrate and grows into CNTs. The catalysts particle stay at one end (top or bottom) of the growing SWCNT (Fig. 6a). Adding plasma to a CVD process allows to grow SWCNTs in a strictly vertical direction. Despite the huge progress in selective synthesis of SWCNTs, most production routes still produce samples 
with metal and carbon impurities (e.g. amorphous carbon) and contain mixed chiralities, diameters and lengths.

The two most used methods in research are nowadays HiPCO and CoMoCAT 77. In HiPCO process a continuous-flow of gaseous carbon monoxide $(\mathrm{CO})$ under high pressure $(\approx 30$ atm. $)$ acts as a feedstock for $\mathrm{CNT}$ growth and iron carbon monoxide $\mathrm{Fe}(\mathrm{CO})_{5}$ as a catalyst. Generally, HiPCO CNTs have very few structural defects, a wide distribution of $(\mathrm{n}, \mathrm{m})$ values and an average diameter larger than that of CoMoCAT CNTs [74]. CoMoCAT is a catalytic CVD process where $\mathrm{CO}$ decomposes into $\mathrm{C}$ and $\mathrm{CO}_{2}$ at $700-950^{\circ} \mathrm{C}$ in the presence of a special Cobalt-Molybdenum catalyst [78]. This method provides chirality enriched samples with a narrow $d$ distribution of diameters (all of CNTs used in this work are CoMoCAT CNTs). More recently, Jasti and Bertozzi proposed a novel bottom-up organic synthesis of homogeneous CNTs with discrete chirality [73]. They used ring-shaped carbon macrocycles as templates for growing CNTs that will retain information regarding chirality and diameter of the starting piece (Fig. 6b). If this method will be further developed and can produce larger quantities of samples, it will render elaborate purification techniques unnecessary. Until then the purification will remain an important step in chirality enrichment of CNTs.

\subsubsection{Structure and geometry of SWCNTs}

Carbon can hybridize in $\mathrm{sp}, \mathrm{sp}^{2}$, or $\mathrm{sp}^{3}$ and form several distinct allotropes, such as diamond (tetrahedral lattice), graphite (sheets of hexagonal lattice), and fullerenes (spherical or tubular formations) [78]. Graphene is a single sheet of graphite, where all C-atoms are arranged into a honeycomb (hexagonal) pattern with $\mathrm{sp}^{2}$ hybridization. This structure provide a basic for other $\mathrm{sp}^{2}$ carbon nanomaterials. By stacking 1D graphene layers on top of each other in a regular distance of $3.41 \AA$, one gets 3D graphite. Rolling a 1D graphene nanosheet into a hollow cylinder, will result in an 1D carbon nanotube. Stacking multiple layers of graphene into each other, will produce multi-walled carbon nanotubes (MWCNTs). Depending on the number of graphene tubes, MWCNTs have diameters ranging from 2 to $50 \mathrm{~nm}$ and length from $1 \mu \mathrm{m}$ up to a few centimeters [78. For optical sensor applications MWCNTs are not as promising as their single-walled counterparts and this thesis will focus only on SWCNTs. 
a

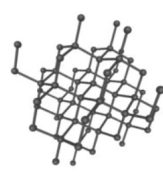

diamond

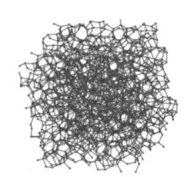

amorphous carbon

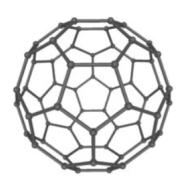

$\mathrm{C}_{60}$ buckminste fullerene

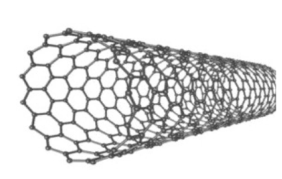

single-walled carbon nanotube graphene

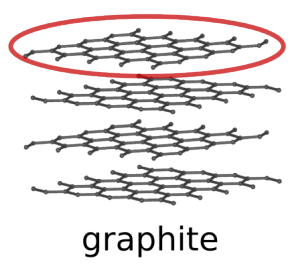

d

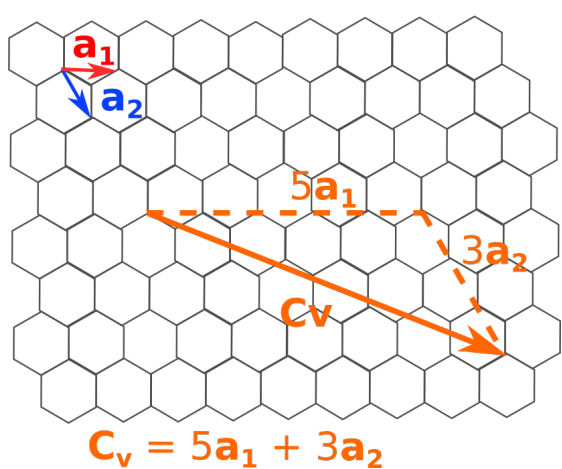

Figure 7: Geometry of various carbon allotropes and SWCNTs (a) Allotrope forms of carbon, (b) Scanning probe microscope image of a $1.3 \mathrm{~nm}$ diameter chiral SWCNT. Adapted with permission from [79], (c) TEM image of a MWCNT containing several SWCNTs. Adapted with permission from [80], (d) Graphene lattice honeycomb structure, with both unit vectors ( $\mathbf{a}_{1}$ and $\mathbf{a}_{2}$ ) and an example of a roll-up vector $\mathbf{C}_{v}$ of a $(5,3)$ SWCNT.

For SWCNT the diameters differ from 0.4 to $2.5 \mathrm{~nm}$, and their axial size is of the micrometer range. Some physical properties of SWCNTs derive from graphene, such as C-C lattice distance of $1.42 \AA 81$. Others, such as chirality and the ensuing optical properties, are a unique feature of carbon nanotubes. In this context chirality has a different meaning for SWCNT than in the organic chemistry. Chirality of a SWCNT can be described based on the roll-up vector of graphene. The chiral index $(n, m)$ assigned to the structure can uniquely define each particular tube. The chiral vector $C_{h}$ is a linear combination of the graphene lattice basic vectors $\left(\mathbf{a}_{1}=\mathbf{a}_{1}=0.246 \mathrm{~nm}\right)$ and determine the geometry of the nanotube.

$$
C_{h}=n \mathbf{a}_{1}+m \mathbf{a}_{2}
$$

The orientation of the chiral vector $C_{h}$ is described by the chiral angle $\theta$, which is defined as the angle between $C_{h}$ and $\mathbf{a}_{1}\left[81\right.$. The chiral angle $\theta$ (with $0 \leq|\theta| \leq 30^{\circ}$, because of the hexagonal symmetry of the graphene lattice) can be calculated from:

$$
\cos \theta=\frac{C_{h} \cdot \mathbf{a}_{1}}{\left|C_{h}\right| \cdot\left|\mathbf{a}_{1}\right|}=\frac{\mathbf{n}_{1}+\frac{\mathbf{n}_{2}}{2}}{\sqrt{\mathbf{n}^{2}+\mathbf{n} \cdot \mathbf{m}+\mathbf{m}^{2}}}
$$


Table 1: Geometric and electrical properties of SWCNTs 82 ,

Physical properties

\begin{tabular}{lll}
\hline C-C distance in graphene lattice & $a_{C-C}$ & $1.44 \AA$ \\
Length of unit vector & $\mathrm{a}_{0}=\sqrt{3} a_{C-C}$ & $2.461 \AA$ \\
Chiral vector & $C_{v}=n \mathbf{a}_{1}+m \mathbf{a}_{2}$ & vary \\
Circumference of a SWCNT: & $L=a \sqrt{n^{2}+n \cdot m+m^{2}}$ & $0 \leq m \leq n$ \\
Diameter of a SWCNT: & $d=L / \pi$ & 0.8 to $2 \mathrm{~nm}$ \\
Optical properties & & \\
\hline Metallic CNTs & $(\mathrm{n}-\mathrm{m})=0$ & $0 \mathrm{eV}$ (no band gap) \\
Semi-metallic & $(\mathrm{n}-\mathrm{m})=3 \mathrm{x}, \mathrm{x} \in \mathrm{N}_{>0}$ & $\approx 1-100 \mathrm{meV}$ \\
Semiconducting & $(\mathrm{n}-\mathrm{m})=3 \mathrm{x}, \mathrm{x} \notin \mathrm{N}_{>0}$ & $\approx 0.5-1 \mathrm{eV}$
\end{tabular}

The chiral vector $C_{h}$ also determines all structural parameters of a SWCNT, such as diameter $d$ or the length of the unit cell and the number of C-atoms in it. The diameter of the nanotube is given by the length of the chiral vector $C_{h}$ :

$$
d=\frac{\left|C_{h}\right|}{\pi}=\frac{\mathbf{a}_{1}}{\pi} \sqrt{n^{2}+n \cdot m+m^{2}}=\frac{\mathbf{a}_{1}}{\pi} N
$$

with $N=\sqrt{n^{2}+n \cdot m+m^{2}}$.

Depending on the chiral vector $C_{h}$, one can distinguish three types of SWCNTs, such as: armchair $(n=m)$, zigzag $(n=0)$, and chiral (all the others) (Fig. 7). Both armchair and zigzag are high symmetry CNTs and are labeled after the shape of their cross-section along $C_{h} . C_{h}$ and, consequently, the integers $(\mathrm{n}, \mathrm{m})$ uniquely define $d$ and $\theta$ and the band gap of a nanotube. The band gap ranges from 0 to $\approx 2 \mathrm{eV}$ causing either metallic or semiconducting behavior. To illustrate how dramatic the slight changes of $(n, m)$ integers can be, one can look at nanotubes with similar diameters and chirality: first, the $(10,10)$ SWCNT contains 40 carbon atoms in the unit cell and is metallic; second, the $(10,9)$ SWCNT, with a similar diameter and $C_{h}$ vector, has 1084 atoms per the unit cell and is semiconducting [81]. Different unit cells will be separately discussed in 4.2 .3 when I will determine the number of sensor units per SWCNT.

\subsubsection{Photophysical properties of SWCNTs}

The electronic properties of graphene have been studied extensively in several excellent works [83], [84], 85], 86]. Therefore, in this section, I will only review the various SWCNT band structure (metallic or semiconducting) that derives from the geometrical specifications and the origins of photoluminescence of SWCNTs. 
As mentioned previously, CNTs are 1D materials. A compound is qualified to be denoted as $1 \mathrm{D}$, when it has a sufficiently large $L / d$ ratio [87]. With nanotubes the ratio can be as large as $10^{4}-10^{5}$. At the same time, by rolling the graphene sheet up, a periodic boundary condition is imposed along the nanotube. The periodicity induces a quantization along the nanotube axes [82. In 3D materials (e.g. graphene), electronic density of states (DOS) is a continuous function of energy. Instead in CNTs, the DOS is quantized and its sharp peaks are called Van Hove singularities (VHS). Van Hove singularities occur at critical points of the Brillouin zone. The size and shape of Brillouin zone, as well as the energies between the Van Hove singularities and the specific boundary condition - all that depend on the chiral vector $C_{h}$. Thus, by selecting different chiralities one can choose the optoelectronic properties of CNTs. Figure 8 shows different shapes of DOS for various 0-3D materials and how it corresponds to optical transitions in SWCNTs.

a

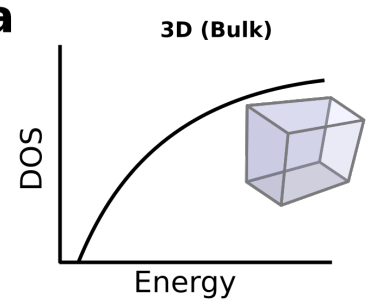

1D (Carbon Nanotube)

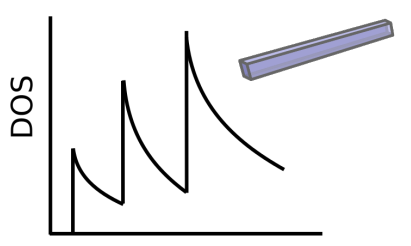

Energy

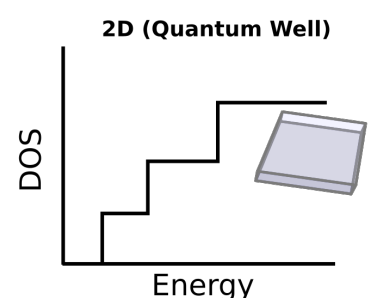

OD (Quantum Dot)

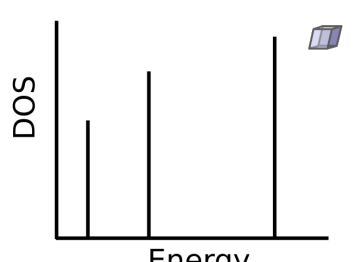

Energy b

Semiconducting

SWCNTs

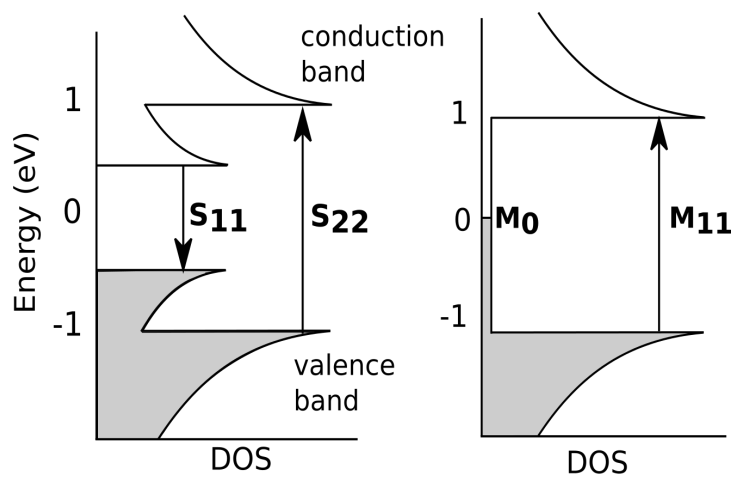

Figure 8: Schematic representation of the electronic density of states (DOS) of various

0-3D materials. (a) DOS of (i) a bulk semiconductor, (ii) a 2D quantum well, (iii) a 1D SWCNT and (iv) a 0D quantum dot. (b) DOS of semiconducting SWCNTs $\left(\mathrm{S}_{11}\right.$ and $\mathrm{S}_{22}$ correspond to the first and second optical transitions) and metallic SWCNTs ( $\mathrm{M}_{0}$ and $\mathrm{M}_{11}$ correspond to the Fermi level and first interband transitions). Schemata are based on theoretical description in $[88]$ and 89

One third of all CNTs are metallic (armchair tubes) and have no band gap between conduction band (the first unfilled energy level at $\mathrm{T}=0 \mathrm{~K}$ ) and valence band (the last filled energy level at $\mathrm{T}=0 \mathrm{~K})$. Semiconducting SWCNTs have a chirality dependent band gap $E_{g}$.

$$
E_{g}=\frac{2 \gamma a_{C C}}{d_{t}} \approx 0.839 \frac{\mathrm{eV} \cdot \mathrm{nm}}{d},
$$


with $\gamma=2.9 \mathrm{eV}$ as the interaction energy between neighboring C-atoms 82 .

The exact nature of electro-optical properties of CNTs is excitonic rather than electronic. When a semiconducting CNT absorbs a photon of the right wavelength (via $\mathrm{S}_{22}$ transition), an electron-hole pair is generated, an exciton. Both electron and hole are attracted to each other by the electrostatic Coulomb forces. This provides a stabilizing energy balance. In the common semiconducting materials (e.g. Si, Ge, etc.) the binding energy of an exciton lies around $10 \mathrm{meV}$. Therefore, the only way to observe absorption to exciton levels is to perform an experiment at extremely low temperatures. In $1 \mathrm{D}$ carbon nanotubes, the electron-hole attraction energy can grow up to $1 \mathrm{eV}$ at room temperature [90]. Metallic tubes do not produce excitonic PL. While their electrons can be excited (and are responsible for the wide optical absorption around $400 \mathrm{~nm}$ ), the generated holes are directly filled by the nearby electrons from the valence band. Therefore, stable excitons cannot be produced. After the exciton was created in semiconducting CNTs, it travels along the nanotubes. For pristine CNTs in the air, the mean exciton diffusion length is reported to be around 610 $\mathrm{nm}$ [91], for CNTs in polymers (e.g. DNA) the mean exciton diffusion length shortens to $\approx 50 \mathrm{~nm}$ [92. When both electron and hole relax via phonon-assisted processes to lower states, they recombine through a $c_{1}-\mathrm{v}_{1}$ transition. This process results in an observed nIR emission and is determined by the radiative lifetime $\tau_{\text {rad }}$. When two excitons collide, or one of them reaches an end of a nanotube, or an unsuitable defect in the $\mathrm{sp}^{2}$ carbon surface, or other disturbances, a non-radiative relaxation pathway occurs (with the lifetime of $\tau_{\text {non-rad }}$ ) and the exciton is quenched 93 .

Thus, generation and recombination of excitons is responsible for the photoluminescence of carbon nanotubes. CNTs have no bleaching or blinking, which is a huge advantage over organic dyes [94]. Extrinsic factors such as inhomogeneities, defects, aggregation, and tube length can affect the non-radiative decay pathways and the experimental brightness of CNT samples [95]. This makes it somehow difficult to determine the intrinsic quantum yield (QY) of carbon nanotubes. In bundles, QY of nanotubes is reported to be in the magnitude of $<0.1 \%$. Whereas in well-separated CNTs samples, the quantum yield can reach up to $1 \%$ [96. Generally, the PL in organic solvents is higher and can rise up to a several percents, e.g. for poly[(9,9-dioctylfluorenyl-2,7-diyl)-alt-co-(6,6'-2,2'-bipyridine)] (PFO-BPy) and toluene mixture the observed QY was up to $2.4 \%$ [97]). Unfortunately, for most biological application SWCNTs must be both bright and soluble in an aqueous environment. From the time-resolved emission spectra the radiative lifetime of CNTs was determined to $\tau_{\text {rad }}$ $=110 \mathrm{~ns}$ 98. This indicates that the low QY values observed experimentally are caused by the strength and the variety of non-radiative decay pathways rather than short lifetimes of radiative decay channel. Consistent with this finding, spectroscopy on single molecules 
showed a significantly brighter CNT emission in the air [99]. So far, the highest reported QY was measured in a SWCNTs dispersion in an aliphatic analog of flavin mononucleotide (FC12) in toluene (QY up to 20\%) 100. The lab of Papadimitrakopoulos proposed that the significant increase in high QY is caused by the extremely tight organization of FC12 around the nanotube. Such tight surface formation excludes oxygen from the surface of SWCNT and increases the QY. In other recent projects, it was reported that adding reducing agents (such as dithiothreitol (DTT) or trolox) to a DNA/SWCNT suspension can increase PL [101], 102]. I will further investigate the interaction between reducing molecules and SWCNTs in 4.1.1. Other possibilities to brighten nanotube PL include removal of solution from the inner volume of SWCNTs [103], or a promising approach of brightening through incorporation of very specific defects in $\mathrm{sp}^{2}$ network of CNT surface. The defects can either trap an exciton between two defects sides [104 or create an new optically allowed defect state (dark excitons) [105]. The latter method can allow to increase the PL up to 28 -fold and red-shift the new emission peak $\left(S_{11}^{*}\right)$ by about $100 \mathrm{~nm}$ from the original excitonic transition $S_{11}$ [106. The experimental condition for those reactions are hard to define and the process is reported to take several days to react, but further investigation in this field might produce SWCNTs with significantly larger QYs than previously believed.

\section{Absorption and emission spectra of SWCNTs}

Absorption spectroscopy is an easy and fast technique to gain information about the state of aggregation and concentration of a CNT sample [107. Since a bare CNT is highly hydrophobic and non water-soluble, a high absorption peak also provides evidence to a successful functionalization. A typical absorption spectrum of SWCNTs shows two intrinsically sharp, structure-dependent transition peaks. First optical transition peak occurs at around $560 \mathrm{~nm}$ (visual range) and second at around $990 \mathrm{~nm}$ (near Infrared, nIR). The exact wavelengths depend on several factors, such as functionalization or aggregation of the sample. Each $(\mathrm{n}, \mathrm{m})$ nanotube has a distinct set of $\mathrm{S}_{i i}$ (respectively, $\mathrm{S}_{11}$ and $\mathrm{S}_{22}$ for first two transition peaks) that can identify nanotube types as a unique molecular fingerprint. The difference between $\mathrm{S}_{11}$ and $\mathrm{S}_{22}$ provide a wide Stokes shift, which is a useful property for spectroscopic application. Bachilo et al. propose following simple linear correlation between a diameter $d$ of a semiconducting nanotube and its optical transition wavelengths [108]:

$$
\lambda_{11}=\frac{h c d}{2 a_{C C} \gamma_{0}}
$$

and

$$
\lambda_{22}=\frac{h c d}{4 a_{C C} \gamma_{0}}
$$


with $d$ as the CNT diameter, $a_{C-C}$ as the distance between C-atoms in a SWCNT network, and $\gamma_{0}$ as the interaction energy between neighboring C-atoms [108]. Following this calculation, CNTs with wider diameters are more red-shifted than CNTs with smaller diameters. This is a good estimation for optical transitions in CNTs, although, in reality the strictly linear correlation is slightly shifted by trigonal warping effects in carbon nanotubes to correct for $(\mathrm{n}, \mathrm{m})$ curvature effects 109 .

There are several extrinsic and intrinsic factors that affect experimental absorption spectra.

\section{Ultrasonication: Surfactant Effects:}

A standard procedure to render highly hydrophobic SWCNTs water soluble is the non-covalent functionalization of a surfactant onto the CNT. For our goal of creating SWCNT-based sensors this step is crucial and will be discussed in detail in 2.2 . Usual proceedings to non-covalently functionalize a CNT with an organic polymer (a so-called 'wrapping' process) require ultrasonication with either an ultrasonic immersion tip or an ultrasonic bath. However, the sonication process causes systematic background elevation that correlates with the duration of sonication. The increased background elevation comes from the increased light scattering of the solution 107. The absorption spectrum will recover the initial background level after a mild centrifugation, which will also separate the pellet (often containing amorphous carbon) from the polymer/SWCNT dispersion. Longer centrifugation times also render more narrow optical transition peaks of polymer/SWCNTs samples.

\section{Impurities:}

Most impurities affect the wavelength range below $600 \mathrm{~nm}$. One of the common contamination of SWCNTs samples is amorphous carbon. It gives absorption background in the near-ultraviolet range due to the strong plasmonic absorption [110], 111]. Metallic nanotubes also have a distinct signature of a strong absorption below $700 \mathrm{~nm}$ and a considerable tail of background extending beyond $900 \mathrm{~nm}$ [107]. This absorption signature results from the $\pi$-plasmon resonance [112]. Complete exclusion of metallic CNTs can be achieved in some cases of polyfluorene (PFO) assisted functionalization [113.

\section{Damage of delocalized $\pi$-system.}

Several covalent functionalizations of SWCNTs damage the $\mathrm{sp}^{2}$ hybridization and introduce $\mathrm{sp}^{3}$ defects into the lattice structure. Localized electronic perturbations introduced into the delocalized $\pi$-system can increase the non-radiative recombination of mobile excitons [114. Intense high power sonication can have a similar effect, damage the $\mathrm{sp}^{2}$ hybridization, introduce unwanted defects and broaden the absorption spectrum. 


\section{Aggregation}

A common problem in both absorption and emission spectroscopy of SWCNTs is the aggregation of single nanotubes into bundles. In bundles CNTs quench their mutual emission, as well as broaden and red-shift the absorption peaks [115]. Aggregation can completely quench intrinsically bright samples. The effect on absorption spectra and on Raman scattering is moderate. To avoid aggregation, it is advisable to use prolonged centrifugation times (e.g. 3x30 min) and low CNTs concentrations (nM-range). The formation of bundles is caused by van der Waals interactions and is especially strong in pristine nanotubes [116]. Functionalization with charged polymers can further assist separation of single CNTs.

a

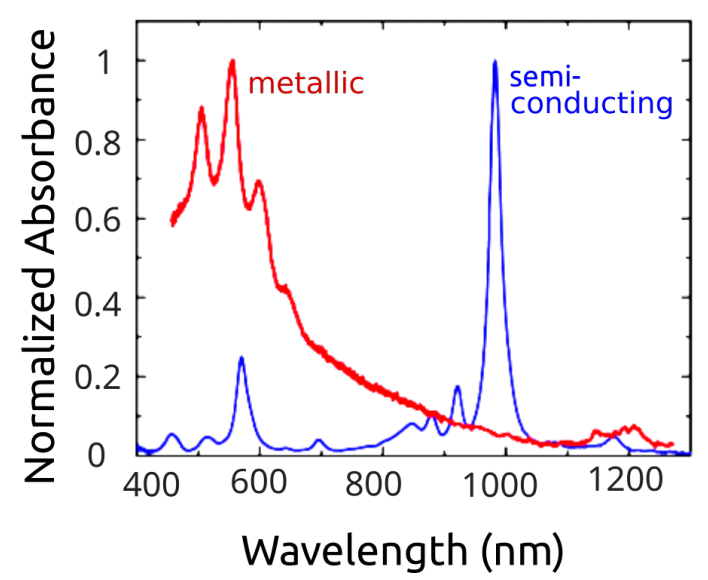

b

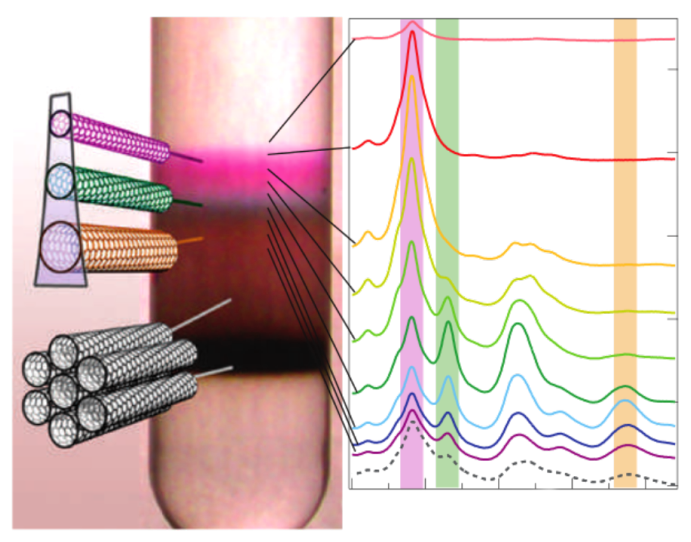

Figure 9: Absorption spectra of SWCNTs. (a) Impacts from metallic SWCNTs (red) on an absorption spectrum of semiconducting SWCNTs (blue). Adapted from [107, (b) A SWCNT dispersion with mixed chiralities exhibit grey color and a broad absorption spectra. The schematic shows how sorting SWCNTs by diameter or electronic properties using density gradient ultracentrifugation (DGU) reveals different colors originated from single chiralities. Adapted with permission from [117.

There are several techniques to not only separate nanotubes and achieve a well-dispersed solution, but also enrich or sort SWCNTs by a required parameter. By far the most wellused technique is the density gradient centrifugation (DGC) or a version of it with higher centrifugation velocity - density gradient ultracentrifugation (DGU) [118]. In general, a layered gradient with increasing iodixanol concentration is prepared and loaded with a sodium cholate/SWCNTs (SC/SWCNTs). Centrifugation (e.g. with 300,000g for 1h) separate different fractions of nanotubes. DGU allows to separate and sort CNTs by their geometry: diameter [119] and length [119] or chirality [120]. Isolation based on bandgap and electronic 
type will separate semiconducting SWCNTs from the metallic ones 117. An ensemble of mixed SWCNTs will appear as a light grey solution. Successful isolation of specific SWCNTs results in a colored solution, with the specific color that depends on the band gap of the extracted SWCNTs [117]. Other techniques include: agarose gel [121 or gel chromatography [122]. There are also purification and separation methods based on special polymers. Special DNA sequences are reported to selectively enrich only one chirality [123]. Polymers with $\pi$-systems are especially efficient in rendering SWCNTs soluble via $\pi$ - $\pi$ stacking. Some of them, such as the aforementioned PFO-polymer, are also known to sort SWCNTs after electric properties and chiralities [113]. Besides PFO, other fluorene-based polymers could achieve similar results [124. The problem with PFO is that it shows the most effective selectivity in toluene or xylene which can not be used for biological application 117.

\subsubsection{Raman spectroscopy of SWCNTs}

Raman spectroscopy is an analytical technique based on Raman scattering. It is commonly used for a characterization of $s p^{2}$ carbon based materials, from 3D graphite to 1D SWCNTs or 0D fullerenes [125]. Raman spectra of carbon materials are well-established and several properties can be observed through all carbon phases. With smaller diameters of SWCNTs, the deviations from the graphene model become larger.

Raman scattering measures the intensity and frequency of photons after they are inelastically scattered from a sample. The incident photons are scattered due to the vibrational or rotational transitions between adjacent atoms. $\Delta E$, the difference between incident and scattered light, provides information about the vibrational or phonon modes, symmetries, chemical composition, phase transition and molecular structures of the material under investigation. In SWCNTs, optical transition energies of the excitation lie in the visible spectral range $(500-600 \mathrm{~nm})$. This coincides with the excitation energies provided by the most lasers and enhances normal Raman scattering to the Resonant Raman Scattering (RRS). The Raman scattering probability in common materials is quite low, only about $1: 10^{7}$. In case of nanotubes the probability of RRS rises up to $1: 10^{3}$ [82]. The enhancement effect allows for unconventionally manifold uses of RRS, such as providing vibrational and crystallographic information, information on electronic states, phonon energy dispersion, or diameters/chirality of SWCNTs in solutions. One especially useful advantage of RRS over other optical characterization techniques (such as UV-vis or emission spectroscopy) is its ability to investigate both semiconducting and metallic SWCNTs, and the fact that it can provide information about a sample regardless of its aggregation state (bundles or single nanotubes) or number of defects [86], 126]. 


\section{Low energy regime of Raman scattering}

In the low-energy range of $100-400 \mathrm{~cm}^{-1}$ one can observe several peaks that result from the radial-breathing modes (RBM) of SWCNTs. This modes are important for identification of nanotubes with specific chirality and diameters. The mode arises from a symmetric $\left(A_{1 g}\right)$ contraction of the cylindrical tube. It also experiences van-der-Waals contributions from neighboring SWCNTs. It is a unique mode for SWCNTs and fullerenes, and does not exist in other carbon materials, such as graphite or diamonds. The RBM peak frequency is inversely proportional to the diameter/chirality of SWCNTs. In armchair SWCNTs, this mode is purely radial. In other SWCNTs the axial eigenvector of vibration has a small contribution, three orders of magnitude smaller than the radial component 126. RBM peaks provide a unique SWCNT fingerprint because each peak can be assigned to the corresponding SWCNT indices. The RBM frequency $\left(\omega_{R B M}\right)$ can be described as:

$$
\omega_{R B M}=\frac{\nu_{B}}{2 \pi c d}
$$

with the velocity $\nu_{B}$ :

$$
\nu_{B}=4 c_{t} \sqrt{1-\frac{c_{t}}{c_{l}}} \approx 42.8 \mathrm{~km} / \mathrm{s}
$$

The constants $c_{t}$ and $c_{l}$ are derived from the transverse and longitudinal sound velocities $\left(C_{66}\right.$ and $\left.C_{11}\right)$ divided by the density of graphite $\left(\rho=2.66 \mathrm{~g} / \mathrm{cm}^{3}\right)$ :

$$
\begin{aligned}
& c_{t}=\sqrt{\frac{C_{66}}{\rho}}=14.0 \mathrm{~km} / \mathrm{s}, \\
& c_{l}=\sqrt{\frac{C_{11}}{\rho}}=21.7 \mathrm{~km} / \mathrm{s},
\end{aligned}
$$

Inserting both transverse and longitudinal sound velocities and the density of graphite yields:

$$
\omega_{R B M}=\frac{227 \mathrm{~cm}^{-1} \cdot \mathrm{nm}}{d},
$$

with $d$ as the SWCNT diameter. For a single chirality, there would be only one peak. For different chiralities, there would be several peaks and their frequency will correlate to a specific SWCNT diameter/chirality, as shown in Figure 10. 
a

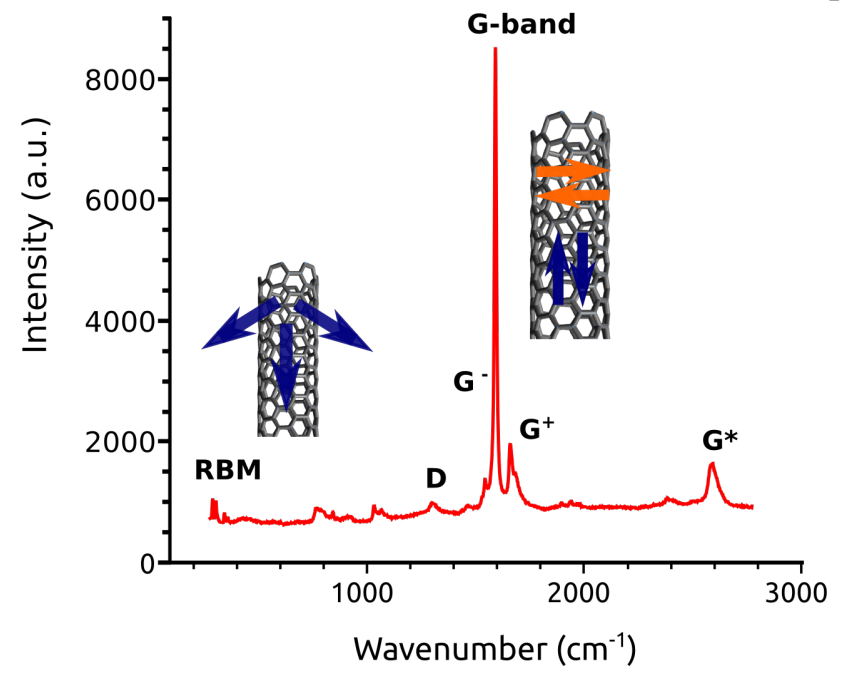

b

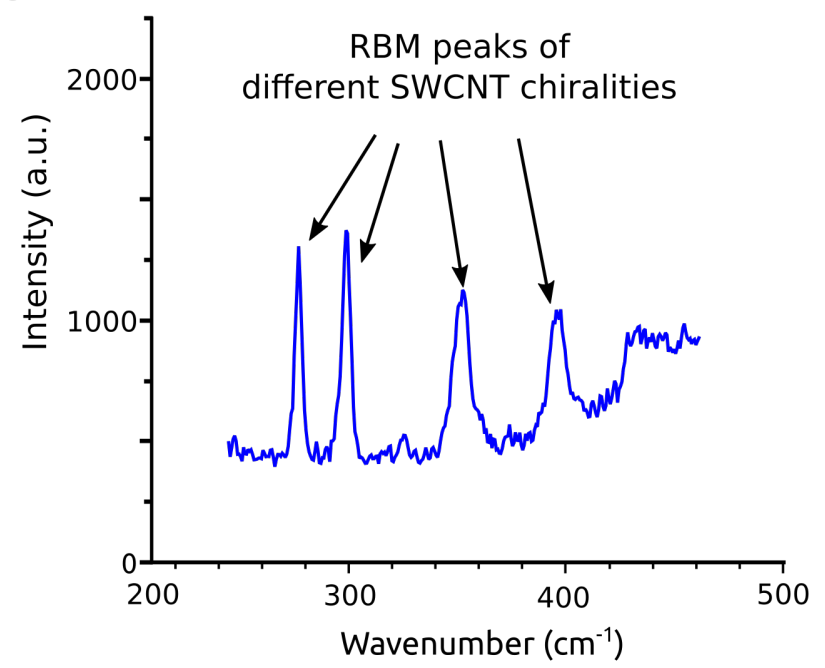

Figure 10: A typical SWCNT Raman spectrum. (a) Raman spectrum with the primary vibrational modes: the RBM, D-, G- and G*-band, (b) An example of radial-breathing modes $(\mathrm{RBM})$ of a SWCNT sample, $\left(\lambda_{e x}=785 \mathrm{~nm}\right)$.

\section{High energy regime of Raman scattering}

In contrast to RBMs, other features of a resonant Raman spectrum of SWCNTs arise from a certain structural similarity to graphite 126 .

- The D-band is an out-of-plane bending mode and occurs at around $1300 \mathrm{~cm}^{-1}$. In a perfect graphene lattice, this mode is normally Raman-inactive. However, when some kind of disorder is introduced into the electric structure, the D-band becomes active. The peak intensity increases with the degree of defect. The defect/disorder can be generated by chemical functionalization, oxidation of SWCNT's sidewalls, physisorbed molecules or any other occurrence that leads to a localization of electron density in the normally delocalized $\pi$-surface. The ratio of $\mathrm{G} / \mathrm{D}$ peak intensities can be used to quantify the number of defects on the SWCNT surface and the percentage of covalent functionalization 127.

- An intense G-band vibration, which occurs at around $1600 \mathrm{~cm}^{-1}$, is present in all $\mathrm{sp}^{2}$ hybridized carbon forms. This mode corresponds to planar, C-C stretching vibrations. In graphene this mode is isotropic, in SWCNTs anisotropic. The inequality of the two different stretching directions arises from the SWCNT curvature and results in splitting of the graphene G-band into two peaks $\left(\mathrm{G}^{+}\right.$and $\left.\mathrm{G}^{-}\right)$. Splitting pattern and the signal intensity can provide information about electric and geometric properties of 
SWCNTs. While the $\mathrm{G}^{+}$peak (high-energy branch) remains mostly invariant, $\mathrm{G}^{-}$can shift to lower frequencies for smaller diameter or metallic SWCNTs, with shifts up to $100 \mathrm{~cm}^{-1}$ 125., 81.

- The mode with the highest energy is the second-order $\mathbf{G}^{*}$-band (also known as G'band). It occurs at around $2600 \mathrm{~cm}^{-1}$ and despite its misleading nomenclature has nothing to do with the G-band. Instead, it is the second-order scattering process of the D-band. It is present even in defect-free SWCNTs, where the D band is completely absent. It is sensitive to small changes in the electronic band structure, such as electron doping, and SWCNT diameter [82]. 


\subsection{Functionalization strategies}

In the previous section, we paid more attention to the pristine SWCNTs and their properties. However, the most interesting qualities of SWCNTs require a functionalization with an organic molecule or a polymer. Not only the water solubility, which is an essential step for biological application, but also the sensitivity to an analyte can only be achieved after a functionalization. It was shown that addition of dopamine to pristine SWCNTs does not affect their fluorescence in any manner [54]. However, after functionalization of ssDNA onto the surface of SWCNTs (a process also called 'wrapping'), the fluorescence will response to dopamine adding, thus creating a sensor. There is a variety of excellent reviews on the topic of chemical functionalization of SWCNTs [128], [129], [130], 131], [132, [133]. One can distinguish between two major types of functionalization: (1) covalent (e.g. oxidation, nucleophilic or electrophilic addition, acid cutting, halogenation, reductive coupling, etc.) and (2) non-covalent (encapsulation, polymer-wrapping, etc.), as shown in Fig. 11 .

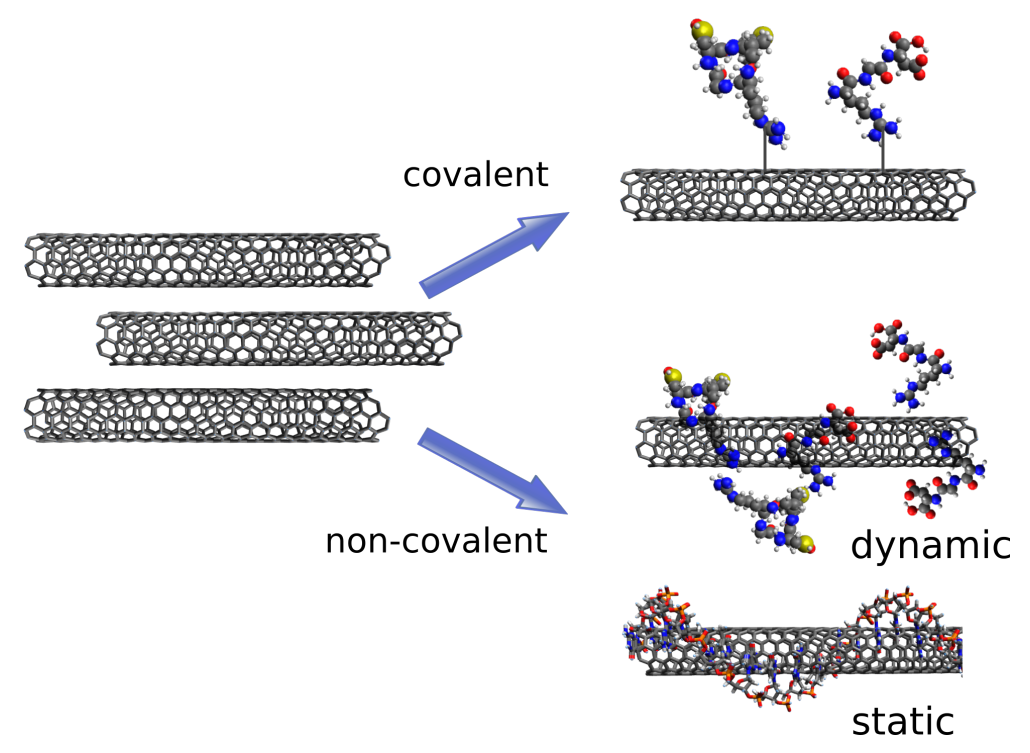

Figure 11: Functionalization strategies. Schematics of covalent and non-covalent functionalization strategies for polymer/SWCNTs.

The unique photoluminescence of SWCNTs requires an intact $\mathrm{sp}^{2}$ hybridization network. Non-covalent functionalization often destroys the delocalized $\pi$-system and introduces defects. If these defects are too frequent on the surface of the nanotube, they can quench the PL. In that case one can observe a broad absorption spectrum instead of distinct peaks 93. Though lately, the defects and the non-covalent functionalization have experienced a renewed attention. Specific defects can even increase the fluorescence of SWCNTs [104], 105], [106] (this was also discussed in 2.1.3). Nevertheless, in this section we will focus on non-covalent functionalizations for sensor applications. 
Non-covalent functionalizations are achieved via (1) enthalpy-driven interactions (mostly $\pi-\pi$, but also $\mathrm{CH}-\pi$ or $\mathrm{NH}-\pi$ ) between the SWCNT surface and the wrapping agent and/or (2) entropy-driven effects, such as hydrophobic interaction in case of amphiphilic surfactants [134]. Tensides and ssDNA are both typical SWCNT surfactants. To achieve a stable ssDNA/SWCNT suspension $10 \mathrm{~min}$ sonication time is enough; for most tensides the same result requires several hours of sonication. The prolonged sonication time itself is not a major problem (apart from the fact that it can alter or damaged the surface of SWCNTs if conducted too excessive). But a general drawback of most tensides as wrapping agents lies in two facts: First, the surfactant molecules are in a dynamic equilibrium with the SWCNT surface. That means they are easily removed from the SWCNT surface by filtration or dialysis which would cause the recurred aggregation and precipitation of SWCNTs [135]. Second, such surfactants need a critical micelle concentration to ensure SWCNT solubility. That implies that residues of surfactants have to be present in solution to guarantee a stable polymer/SWCNT suspension. This could hinder some in vitro experiments, might affect or interact with the analyte, or lead to the denaturation of proteins [136. It is possible to exchange the surfactants if the new polymer has a more stable polymer-SWCNT interaction. That can be achieved e.g. via dialyse. Exchange from SC- to ssDNA-dispersed SWCNT solution can be observed via shifts in the absorption spectra [137]. Various ssDNA sequences also exhibit different affinities to the SWCNTs surface. Iliafar et al. measured this affinity by pulling single-stranded DNA sequences from the SWCNTs surface and determining the applied force [138]. Based on their calculation they established following ranking for binding strength of different homopolymeres: $\operatorname{poly}(\mathrm{T})>\operatorname{poly}(\mathrm{A})>\operatorname{poly}(\mathrm{G}) \geq \operatorname{poly}(\mathrm{C})$, with values ranging from 11.3 to $7.58 k_{B} T$ per nucleotide.

\subsubsection{SWCNT-based sensor applications}

High fluorescence sensitivity makes polymer/SWCNTs complexes perfect basic units for sensor application. Indeed, a lot of polymer/SWCNTs biosensors are known to detect reactive oxygen species (ROS), neurotransmitters (e.g. dopamine, epinephrine, etc.), small molecules (NO, ATP, $\mathrm{H}_{2} \mathrm{O}_{2}, \mathrm{NH}_{3}, \mathrm{H}_{2}$, etc.), and small peptides/proteins [58], [62], [139], [140], [141]. At this point it is necessary to add that the labeling of a ssDNA/SWCNTs unit as a 'sensor' might be not entirely correct. Chemical sensors consist of both chemical recognition and signal transduction parts. It provides information about changes in the environment in a measured parameter (output). A probe is only a molecular probe, an indicator without an analytical signal, and is in most cases not ready to use. Although, the contemporary scientific community uses these two terms interchangeably [142]. Therefore, in this thesis there is also no differentiation between a sensor and a probe, and the ssDNA/SWCNTs or other polymer/SWCNTs are labeled as sensors. 
Regardless of the exact biological purpose (nIR staining, delivery of drugs into a cellular environment, etc.) one crucial characteristic of a new bio-material is its toxicity. At first, SWCNTs were believed to be as harmful after their inhalation as asbestos [143], [144]. It was soon discovered that the toxicity of CNTs depends strongly on contributing factors, such as contamination, length of CNTs, functionalization, dose and exposure, and presence of metallic catalyst residues [145] [146], [147], [148]. Long nanotubes (mostly MWCNTs with length $>20 \mu \mathrm{m}$ ) can indeed cause pulmonary damage, while shorter nanotubes do not exhibit the same harmful effects [149]. It should also be considered that nanotubes for most sensor application are either tightly bound in a hydrogel or suspended in an aqueous solution so that no airborne risks are present. So far, conjugated protein- or peptide/SWCNT complexes showed higher biocompatibility and less immune or acute inflammatory responses than pristine SWCNTs [150]. The challenges of peptide/SWCNT functionalization make such dispersions rare but highly promising [151. The non-covalent functionalization with polyethylene glycol (PEG) also lowers the immunogenicity of the SWCNTs [152]. At the same time, it prevents the non-specific phagocytosis by the reticuloendothelial system (RES), thus, prolonging the duration of PEG/SWCNT circulation in the blood system [153]. In addition to enhancing their biocompatibility and decreasing the cytotoxicity, functionalization of SWCNTs provides analyte-specific interactions.

\subsubsection{Functionalization and selectivity}

Single stranded DNA sequences are often used as an organic phase for SWCNT-based sensors [58. The specificity of ssDNA/SWCNT sensors depends on the ssDNA sequence. For example, a high-throughput screening approach showed that $(\mathrm{AT})_{15} / \mathrm{SWCNTs}$ are useful for NO detection [154, while $(\mathrm{GC})_{15} /$ SWCNTs provide an excellent sensor for norepinephrine in nanomolar range [57]. Other analytes of various ssDNA-sequences include NADH, L-ascorbic acid, dopamine, and riboflavin [155]. It is also possible to use an aptamere (DNA or peptide sequences that binds to a specific target molecule), 'wrap' it around a SWCNT and detect the aptamere target molecule [156]. Nitric oxide (NO) (which i. a. plays an important role in signaling in the CNS 157]) can be detected by 3,4-diaminophenyl dextran (DAP-dex)-functionalized SWCNTs [155. The detection limit of these sensors goes down to $70 \mathrm{nM}$. The exact location of NO production in cells can provide insight into its biological function [158]. DAP-dex/SWCNTs sensors were able not only to detect endogenously produced NO inside the macrophage cells but also provided spatial information about their release sides.

Most of these sensors are based on the intensity change upon binding of an analyte. 
Even more reliable are sensors which report a shift of the emission wavelength. Such a shift (hypsochromic) of $2 \mathrm{meV}$ was observed after adding of the oligonucleotide sequence complementary to the one wrapped around SWCNTs [159]. A year later it was shown that the wavelength shift was indeed by the DNA hybridization [160. Here, the DNA around the SWCNT and the complementary DNA strand in the solution were both conjugated to a donor and an acceptor fluorophore, respectively. As soon as the complementary DNA strand was introduced into the system, a decrease in the donor emission and a subsequent increase in the acceptor emission were observed simultaneously. Interestingly, the kinetics of DNA hybridization around SWCNTs were several orders of magnitude lower than the kinetics of DNA hybridization in solution (10 minutes vs 13 hours, respectively). The detection limit of the complementary DNA strand goes down to $6 \mathrm{nM}$.

\subsubsection{Sensitivity and detection limits}

\section{Detection limits of ssDNA/SWCNT}

ssDNA functionalized SWCNTs have proved to be excellent sensors to detect neurotransmitter via emission intensity change [64]. Specifically, the nIR fluorescence of these sensors would increase in the presence of catecholamines. Normalized intensity increase can go up to $200 \%$ upon addition of $100 \mu \mathrm{M}$ dopamine to ssDNA/SWCNTs [55]. The strength of the increase depends on the SWCNT chirality $(n, m)$, but usually all chiralities react in the same manner to the same analyte, e.g. an increase in case of dopamine, a decrease in case of serotonin [54]. Similar findings were reported for a single molecule ssDNA/SWCNTs immobilized on a surface 57] (Fig. 12a). In a screening approach it was possible to identify (GA) 15 as the most promising ssDNA sequence for dopamine detection [56]. Analyte sensitivity for this sequence does down to the single-molecule level (100 pM) for individual nanosensors and lies in the single-digit nanomolar range for nanosensors arrays or experiments in solutions. A dopamine calibration curve showed a linear regime for intensity change between $10 \mathrm{nM}$ and $10 \mu \mathrm{M}$. This concentration range almost covers the biological neurotransmitter concentration ranges. It can detect both concentrations of $\mathrm{mM}$ dopamine (1 ms after the release) and $\mu \mathrm{M}$ (after $100 \mathrm{~ms}$ ). On the other hand, it also shows a nM-sensitivity essential to track the concentration gradient of the neurotransmitter diffusing away from the release point. At concentrations of dopamine over $10 \mu \mathrm{M}$ the sensor is saturated and the response does not increase any further. It is important to keep in mind that dopamine can be easily oxidized to dopamine quinone or gradually polymerized to polydopamine [161, 162. Higher concentrations are more prone to polymerization. For example, an initially colorless and clear millimolar dopamine solution shows evidence of polymerization in form of yellowing and darkening within the first hour. Smaller and faster changes might still be undetectable 
with bare eye. This might interfere with the observed intensity change at higher concentrations or time-dependent variations in the absolute intensity, as the polydopamine can quench SCWNT fluorescence.

\section{ssDNA/SWCNT sensor kinetics}

Another useful characteristic of ssDNA/SWCNTs sensors is their reversibility. This was shown by immobilizing ssDNA/SWCNT sensors on a glass surface of a flow chamber 54. Before that, the glass was treated with (3-Aminopropyl)triethoxysilane (APTES) to functionalize it with positively charged amino groups and ensure stable immobilization of negatively charged ssDNA/SWCNTs. The sensors were then periodically exposed to dopamine concentration of $100 \mu \mathrm{M}$ and washed with PBS. The observed PL intensity increased up to 5-times after the analyte addition and returned approximately to the starting level after washing (Fig. 12b). A slight increase in the base intensity could be avoided by extending the washing procedure over $30 \mathrm{~min}$. The PL increase was instantaneous and the recovery of the half-maximum intensity after washing was in the seconds-range $(49 \pm 34 \mathrm{~s}$, for $\left.(\mathrm{GT})_{15} / \mathrm{SWCNTs}\right)$. The $k_{\text {off }}$ values could be convoluted by a slow process of dopamine washing out of the system and might be shorter for real events.
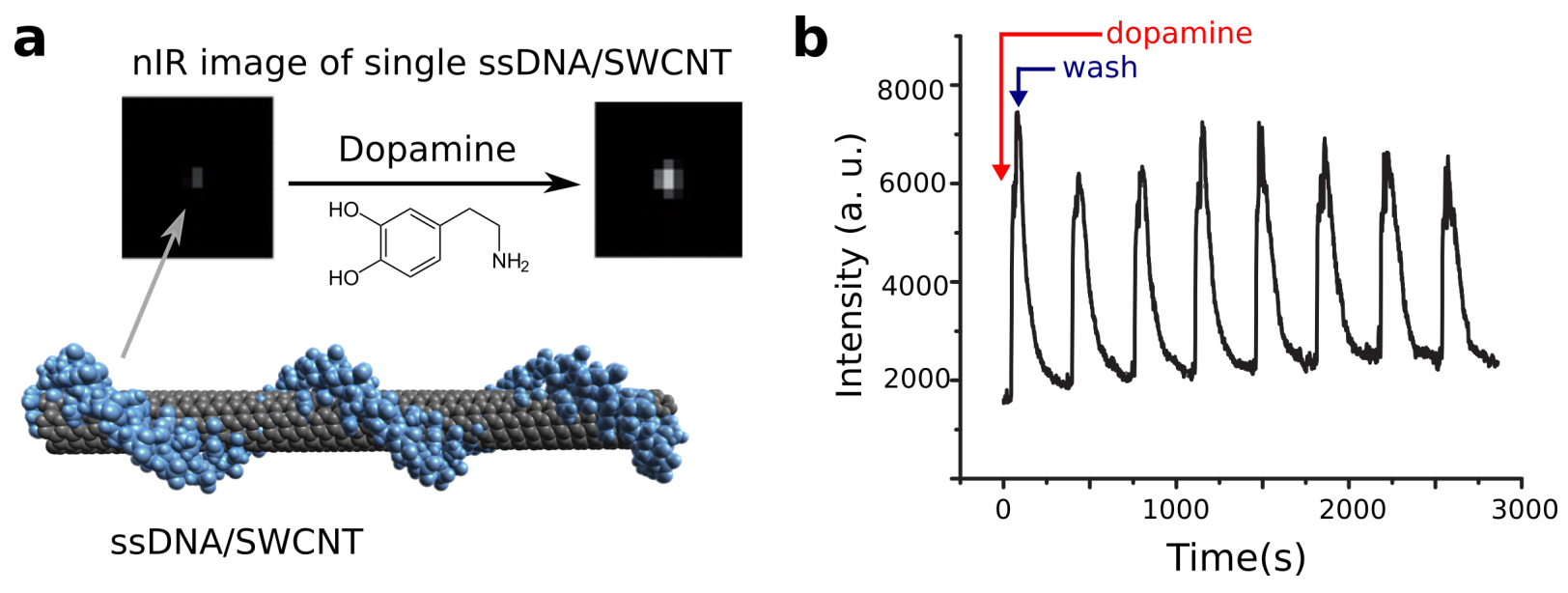

Figure 12: ssDNA/SWCNT complex as a dopamine sensor. (a) Schematic of a ssDNA/SWCNT sensor for dopamine detection, with nIR image of a single molecule of $(\mathrm{GT})_{15} / \mathrm{SWCNT}$. After addition of dopamine $(100 \mu \mathrm{M})$ an increase in fluorescence could be observed, (b) Reversible emission increase of (GT) ${ }_{15} /$ SWCNT sensors immobilized on the surface. Modified and adapted from [54].

Later, the dissociation constants $k_{d}$ were evaluated in solution for a library of ten ssDNA 
sequences [57]. $k_{d}$ values span a range between $\mathrm{nM}$ and $\mu \mathrm{M}$, depending on the ssDNA sequence and analyte molecule: $2.3 \mathrm{nM}$ for $(\mathrm{GC})_{15} / \mathrm{SWCNTs}$ for norepinephrine and $9.4 \mu \mathrm{M}$ for $(\mathrm{AT})_{15} / \mathrm{SWCNTs}$ for dopamine. The span of several orders of magnitude emphasizes the importance of the organic phase, in this case the oligonucleotide sequence. $k_{d}$ values determine the dynamic range of the sensor and in consequence its biological relevance. Theoretical MD simulations of binding and unbinding events onto the SWCNT revealed that fast binding (in the magnitude of $k_{o n}=10^{7} \mathrm{M}^{-1} \mathrm{~s}^{-1}$ ) and slow unbinding constants $\left(\approx k_{o f f}\right.$ $=1 \mathrm{~s}^{-1}$ ) would result in a saturated response image and make any diffusion tracking impossible [22]. On the other hand, if the sensor is too slow $\left(k_{o n}=10^{3} \mathrm{M}^{-1} \mathrm{~s}^{-1}\right)$ it will miss the fast analytes and observe only stochastic events. For tracking of diffusing neurotransmitters after their release, a sensible sensor should have fast binding kinetics and rather slower unbinding. $k_{o n}=10^{6} \mathrm{M}^{-1} \mathrm{~s}^{-1}$ and $k_{o f f}=100 \mathrm{~s}^{-1}$ are suggested by the MD simulations. So far, ssDNA/SWCNTs had shown both fast binding kinetics (instantaneous in the experimental setup) and the slow recovery of intensity in the double-digit seconds range.

\section{Peptides as recognition units for SWCNT-based sensors}

Although ssDNA/SWCNTs sensors are highly sensitive, their selectivity still remains a challenge. Proteins and peptides are well-known for their specific molecular interactions in nature. Or, as aptly put by Andrade et al., every protein has its own 'unique molecular personality' 163. So far, peptides were sparsely used to functionalize SWCNTs compared to ssDNA 151. On the one hand, a stable peptide/SWCNTs dispersion is hard to achieve. Not every peptide functionalization guarantees colloidal stability due to a higher tendency of aggregation. On the other hand, an organic phase based on peptide opens new possibilities for incorporating highly specific receptors for sensing. Since I will employ a short peptide sequence in the second part of my thesis, I would like to give a short overview of peptide/SWCNTs complexes in sensor applications.

The simplest approach is again a non-specific adsorption of peptides onto the SWCNT surface 164. Although, it is one of the most commonly used strategies for developing new polymer/SWCNT systems, this approach is not useful for peptide wrappings. To form stable dispersions it is necessary that the $\pi$-stacking, electrostatic, van der Waals, hydrophobic, or steric interactions of wrapping polymers at the SWCNT interface outweigh the attractive forces between single SWCNTs 165. In case of peptides, these interactions are generally weaker than attractive electronic SWCNT interactions. Therefore, peptides often require a specific sequence with a significantly high percentage of polar or aromatic amino acids (such as Phe, Tyr, and Trp) that would adsorb onto the SWCNT surface and stabilize them in an aqueous environment 166. This limits the choice of possible peptide/SWCNTs sensors 
immensely. Another aspect to consider during peptide adsorption is not only the individual amino acid sequence but also the secondary and tertiary structure. 'Wrapping' of peptides around a SWCNT can destroy this structure and consequently the functionality of the peptide. Such was the case for $\alpha$-chymotrypsin (CT) and soybean peroxidase (SBP) after their assembling onto the SWCNT surface [167]. The SBP retained around 30\% activity, while CT exhibited only $1 \%$ of its functionality after adsorption onto the SWCNT surface. The decreased activity for enzymes was attributed to high perturbation in the secondary peptide structure. SBP contains 19 hydrophobic residues that could lead to favorable interactions with SWCNT. All of those are located on the surface of the protein and an attachment to SWCNT would disturb them only marginally. On the contrary, in CT the hydrophobic parts are located inside the protein. To interact with the hydrophobic SWCNT surface, it needs to partially unfold, thus destroying the protein functionality. Peptides have a slightly higher tendency to adsorb onto chiral or zigzag rather than armchair SWCNTs [168, [169]. That might help in case of an unfavorable native distribution. A different $\mathrm{pH}$ can also change the net charges of the amino acid residues, thereby changing the interactions between the peptide and the SWCNT [170]. Nevertheless, the adsorption of peptides onto the SWCNTs is highly limited.

\section{Specific hybrid polymer conjugation}

For sensor applications it is most crucial to preserve the intrinsic selectivity of the peptide after assembling to the SWCNT. However, as established, the non-specific physical adsorption onto the hydrophobic SWCNT surface often disrupts the functionality of the protein by disturbing the secondary structure [167]. An alternative and more elaborate hybrid strategy offers more control over protein conformation and preserves the peptide functionality. Here, a biological polymer is first non-covalently adsorbed onto the SWCNT surface and then covalently conjugated to the peptide. A successful example of this strategy is presented in the rational design of the glucose sensor by Yoon et al. [171]. A stable, optical glucose sensor for continuous blood sugar monitoring would improve the life standards of diabetes patients 172 . It is important that the sensors work for long time periods and are highly specific. In their glucose sensors design, Yoon et al. first functionalized SWCNTS with carboxylated polyvinyl chloride (cPVA) and then conjugated it to the glucose binding protein (GBP). GBP is a a part of a family of binding proteins (BPs). They are present in the periplasmic space of gram-negative bacteria (such as Escherichia coli) and transport specific small molecules from the extracellular space to the membrane receptors [173]. Known BPs include proteins for glutamine, arabinose, histidine, and glucose (with binding constants in $\mu \mathrm{M}$ range) [174]. Those BPs have an advantage over enzymes since they are not limited by enzyme degradation process and could function long periods of time. In contrast, for glucose 
oxidase based SWCNT sensors the presence of sufficient amounts of oxygen is a limiting factor 175]. Moreover, glucose oxidase sensors produce hydrogen peroxide that accumulates and degrades the enzyme over time. On the contrary, GBP does not process glucose. The binding is a dynamic association between the substrate (glucose) and the protein (GBP). Upon binding to the analyte, the GBP undergoes a conformational change. Two domains of GBP close around the glucose molecule. The change affects the chemical surrounding of the SWCNT surface and results in a highly selective and reversible fluorescent quenching. The PL quenching is proportional to the glucose concentration. This strategy allows to monitor glucose concentrations in real-time and for up to 60 hours. A further development of this strategy might allow to implement the sensors subcutaneously or in a point-of-care device.

\subsubsection{The integrin binding motif RGD}

To enhance the selectivity of polymer/SWCNT sensors, we want to expand the organic phase beyond simple polymer wrappings. Therefore, we will introduce a hybrid strategy with several individually adjustable units. Proteins and peptides are well-known for their specific molecular interactions in nature, therefore, it would be advisable to employ them as recognition units. As a proof of principle for future application, we will focus on a peptide sequence well established as a recognition unit - the integrin binding motif. There are different types of integrin, but most of them bind to the common short peptide sequence Arg-Gly-Asp (RGD). The conformational geometry of the RGD motif determines how strong and to which integrin it binds.

Integrins are transmembrane receptors that mediate cell-adhesion, modulate signaling cascades in control of cell motility and the intercellular communication [176]. As extracellular matrix (ECM) adhesion receptors they are responsible for both outside-in and inside-out signaling cascades. In the first case, they respond to the environmental cues (e.g. adjust their cytoskeletal structure, shape and motility to the environment) and in their second capacity, they forward cell-internal signals to the ECM [177]. In addition to its role in cell adhesion, integrins also participate in signaling events that govern epithelial differentiating and play a pivotal role in the platelet-mediated blood clotting [178], [179]. Currently known integrin family is formed through the non-covalent association of $\alpha$ and $\beta$ subunits [180]. Their cytoplasmic domains are generally short (about 40 to 70 amino acids). The class of $\beta_{4}$ subunits is an exception with its cytoplasmic domain of over one thousand amino acids. The exoplasmic domains are usually longer (about 20-25 nm) and the last quarter of it forms the ligand-binding region that reaches out in the ECM [181]. Usually, the binding occurs in the binding pocket between the two subunits. The schematics of an integrin complexes is shown in Fig. 13. Both subunits are also known to bind various divalent 
cations, which may be directly involved in the binding events or stabilize the 3D structure of the protein [182]. Currently, there are 24 known variations of the two integrin subunits, each of them are specified for different tasks. For example, the class of integrins involving $\beta_{b 3}$ subunit is prevalent on platelets and implicated in hemostasis and thrombosis 183. Lately, this integrin class has received renewed attention in drug discovery efforts 184. Selective integrin ligands target integrin-overexpressing cancer tumors or help in preventing excessive blood coagulation or thrombotic complications after coronary interventions 185].

a

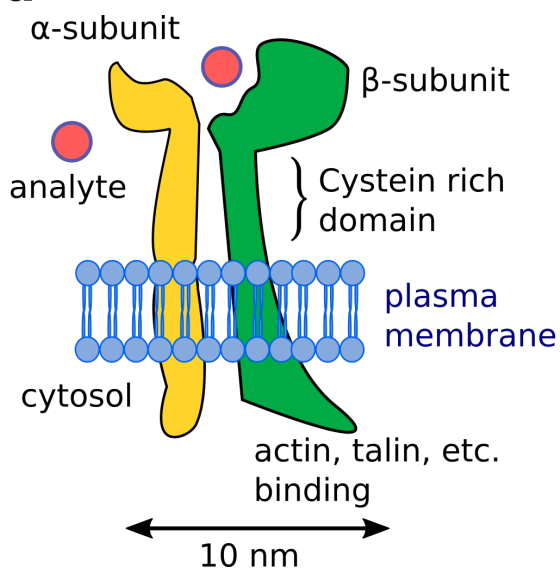

b

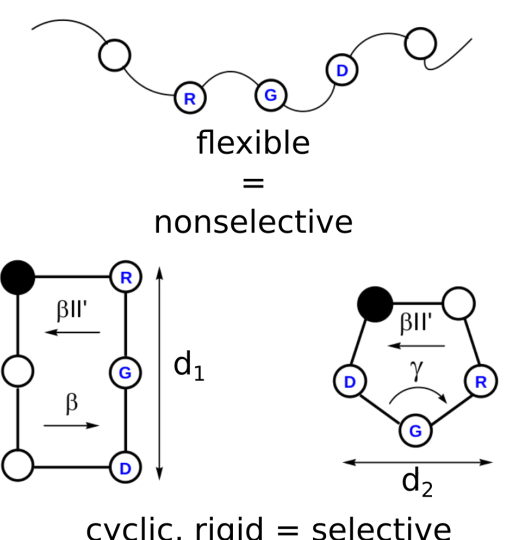

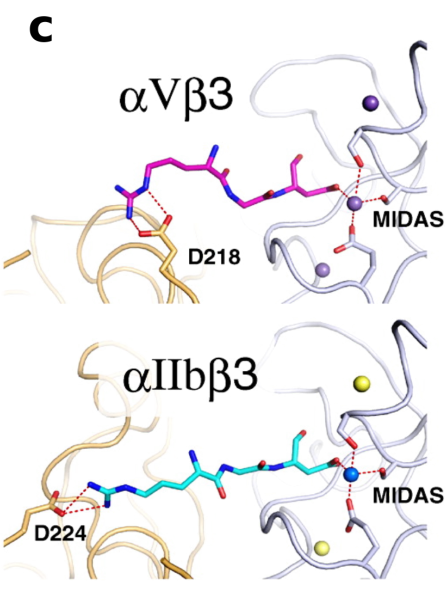

Figure 13: Integrin complex and possible RGD binding motif geometries.(a) Schematics of an integrin complex, with both subunits and the various domains, (b) Illustration of a flexible linear RGD peptide vs. conformational restricted geometries. The distance between Arg and Asp amino acid side chains is denoted as $d$. The smaller $d$, the more kinked is the RGD motif. Adapted from [186], (c) Crystal structures of different integrin types $\left(\alpha_{v} \beta_{3}\right.$ (top) and $\alpha_{I I b} \beta_{3}$ (bottom)) in complex with RGD ligands. Adapted from 187 .

In 1984, Pierschbacher and Ruoslahti discovered that the shot peptide sequence of ArgGly-Asp amino acids (RGD) acts as the minimal integrin binding motif in fibronectin [188]. Later, 8 out of 24 integrin types were shown to recognize the RGD-motif that can be found in their target proteins (fibrinogen, vitronectin, von Willebrand factor, collagen, etc.) [189]. Yet despite their apparent similarity, integrins can distinguish between those different proteins. What governs their selectivity is the geometrical conformation of the RGD motif inside different proteins. The presence and chemical nature of flanking residues of the RGD-core sequence affect the conformation and therefore the integrin selectivity and binding strength. The group of Kessler was able to identify structural and geometrical factors of various RGDmotifs responsible for selective binding to different integrin types [190.

The spatial conformation of the RGD motif determines how strong and to which integrins it 
binds. For example, fibronectin recognizes both $\alpha_{5} \beta_{1}$ and $\alpha_{I I b} \beta_{3}$, but has a stronger affinity to the former type. On the other hand, the $\alpha_{I I b} \beta_{3}$ integrin is primarily expressed on blood platelets and preferentially binds to fibrinogen, prothrombin and plasminogen [179]. At the same time, short linear peptides outside of the scaffolding protein structure exhibit a significantly lower binding to the $\alpha_{I I b} \beta_{3}$ protein. Thus, a prearranged conformation outweighs the advantages of a conformational freedom, such as e.g. easier access into the integrin's binding pocket (as shown in Fig. 13b). A stretched peptide geometry with the highest distance ( $1 \mathrm{~nm}$ ) between the positively-charged arginine residue and the carboxyl group of the aspartate residue exhibits high affinity to the $\alpha_{I I b} \beta_{3}$ integrin [191. In contrast, an RGD-motif with bent or kinked geometry prefers the $\alpha_{5} \beta_{1}$ integrin type (Fig. 13k). One way to ensure preferred affinities is to synthesize a cyclic RGD peptide with rigid spacial conformation. Via spatial screening Kessler et al. found a peptide sequence with higher selectivity for the $\alpha_{v} \beta_{1}$ than for the $\alpha_{I I b} \beta_{3}$ integrin type. They achieved selectivity by incorporating either a proline or a D-amino acid into the RGD peptide-ring [192]. It shows that small changes in the peptides flanking the RGD sequence have a drastic impact on the selectivity and affinity pattern of RGD recognition units 193. 


\subsection{Near-infrared fluorophores for biomedical imaging}

One important goal of bionanosensor development is to implement these sensors in clinical applications. Biomedical imaging can help to guide surgical treatment, monitor inflammatory sides and treatment efficiency, diagnose diseases and their distribution, and much more [194]. Such techniques rely on deep tissue penetration to investigate structural characteristics and possible 'malfunctioning'. There are two possible pathways to enhance bioimaging development: (1) design new measuring equipment (e.g. confocal microscopy, fluorescence-mediated tomography, etc.) [195], or (2) develop new imaging agents, with improved biocompatibility, photostability and spectral properties [196. While the discovery of new techniques, such as fluorescence lifetime correlation spectroscopy or computer tomography, allowed for a huge leap forward, there is still a lack of similarly revolutionary fluorophores [197], [198]. So far, the progress has been hampered by the fact that most commercially available fluorophores emit in the visible spectral range [199. Unfortunately, it is exactly the same spectral range where most mammalian tissues are opaque [200]. In this section, we will discuss fluorophores with more favorable spectral properties, such as emission in the near Infrared region (nIR). The focus of this chapter will be on the nIR fluorophore Egyptian Blue.

\subsubsection{Scattering in biological tissues}

Imaging techniques are based on the interaction between photons and tissue. When a beam of light interacts with a material under investigation, part of the incoming light is reflected, part of it is transmitted, and part of it scattered [201]. Over 99\% of the scattered light $\left(\nu_{\text {out }}\right)$ has the same frequency as the incident ray $\left(\nu_{i n}\right)$ : those are Mie and Rayleigh scattering. A small percentage of the scattered photons has a different wavelength than that of the incoming beam: these are Raman and Brillouin scattering. Both types are forms of inelastic scattering [202]. Brillouin scattering results from the interaction of light with acoustic phonons and provides information about the elastic properties of the material [202]. Rayleigh scattering results from the interaction with vibration or rotation of atom bonds and provides information about symmetries, chemical composition, phase transition, the vibrational or phonon modes, and molecular structures of the investigated sample 201.

- Mie and Rayleigh Scattering $\nu_{\text {in }}=\nu_{\text {out }}$

- Brillouin and Raman Scattering $\nu_{\text {in }} \neq \nu_{\text {out }}$ 
Whether the light beam will induce Mie or Rayleigh scattering, depends on the particle size:

$$
x=\frac{2 \pi r}{\lambda}
$$

where $r$ is the radius of a spherical particle, and $\lambda$ is the incident wavelength. Scattering regimes are: Rayleigh for smaller particles ( $x \ll 1$, collagen fibrils, membranes, etc.) and Mie for objects within similar dimensions as the light wavelength $(x \sim 1$, vesicles, cells, etc.). For particles with $x \gg 1$, the light is scattered according to their projected area (geometrical scattering, e.g. raindrops) 201. Rayleigh scattering has a strong wavelength dependence $\left(\approx \lambda^{-4}\right)$ and is more effective at shorter wavelengths (visualized in Fig 14 , black line). The intensity $I$ of Rayleigh scattering for an object of diameter $d$ and with refractive index $n$ is given by:

$$
I=I_{0} \frac{1+\cos ^{2} \theta}{2 R^{2}}\left(\frac{2 \pi}{\lambda}\right)^{4}\left(\frac{n^{2}-1}{n^{2}+2}\right)^{2}\left(\frac{d}{2}\right)^{6}
$$

with $I_{0}$ as the starting intensity, $R$ as the distance to the object, $\lambda$ as the beam wavelength, and $\theta$ as the scattering angle. After accounting for all possible angles, one can define the scattering cross-section $\sigma_{\mathrm{s}}$ :

$$
\sigma_{\mathrm{s}}=\frac{2 \pi^{5}}{3} \frac{d^{6}}{\lambda^{4}}\left(\frac{n^{2}-1}{n^{2}+2}\right)^{2}
$$

Following these equations, one can establish that scattering is reduced at longer wavelengths, regardless of the exact tissue. Empirical measurements of scattering in different tissues confirm the inverse proportionality of scattering to the excitation wavelength 203. However, the exponent for the wavelength dependence $\mu_{s}^{\prime}$ varies due to the tissue inhomogeneity and different structures (e.g. subcutaneous vs. bone tissue). The largest exponent value is that for the brain tissue $\mu_{s}^{\prime} / \mathrm{mm}=4.72(\lambda / \mu \mathrm{m})^{-2}$ 204. This indicates the steepest decay of scattering with increasing wavelength and makes nIR emission especially suitable for bioimaging of the brain tissue.

\subsubsection{Advantages of the near Infrared region}

Aside from the unintended scattering processes, there are several other inferences, such as an auto-fluorescent background. Biological samples are rich on endogenous molecules. Often these molecules are also chromophores which can turn excitation light into emission background and heat dissipation [205]. Hemoglobin, which is present in the most biolog- 
ical tissues, is responsible for oxygen transport and absorption of blood in the $<600 \mathrm{~nm}$ range [206]. Besides hemoglobin there are such molecules as reduced nicotinamide adenine dinucleotide (NADH), pigmented cellular structures (such as e.g. reticular fibers, connective collagen tissue, etc.), melanin (natural pigment in human skin), or aromatic amino acids in proteins (such as Try or Phe) 207], 208]. All of them absorb in the ultraviolet and visible ranges. Furthermore, at the wavelengths above $1300 \mathrm{~nm}$ the absorption of water exhibits a number of vibrational overtone bands [209]. That leads to a renewed increase in the absorption background. Hence, the region above the wavelength of high absorption and scattering from biological chromophores, and below the wavelength of absorption of water, is often referred to as the 'biological tissue transparency window' (900-1300 nm) 60]. The different effects are summarized in Figure 14, with blood absorption in red and water absorption in blue.

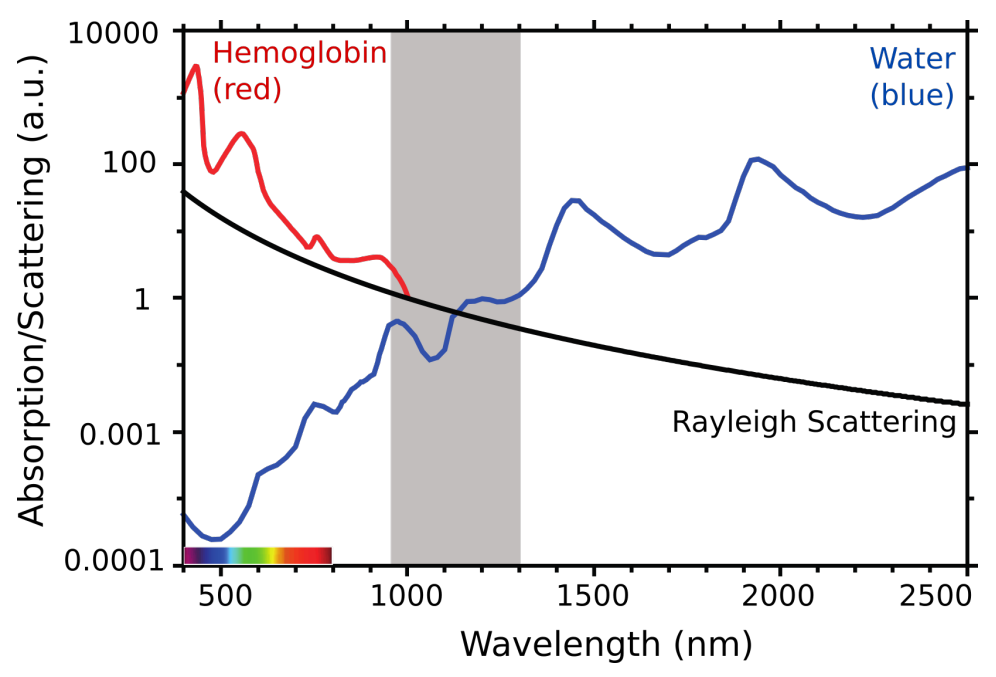

Figure 14: NIR optical window in biological tissues. Absorption and scattering from oxygenated blood (red), water (blue) and scattering (black, $\approx \lambda^{-4}$ ) are plotted against excitation wavelength. The grey area shows the near-infrared tissue transparency window.

To enable deeper tissue penetration without damaging it, an ideal spectral window for biomedical applications should have: (1) no autofluorescence, and (2) low absorption for surrounding structures. The nIR region $(700-1700 \mathrm{~nm})$ seems suitable to this purpose [196]. The nIR range can be further divided into 2 regions: $700-900 \mathrm{~nm}$ range (nIR-I) and $1000-1700 \mathrm{~nm}$ range (NIR-II). The $\mathrm{S}_{11}$ emission peak of CNTs, although dependent on the chirality, lies in the range of $900-1600 \mathrm{~nm}$ [115]. In this region, the absent autofluorescence outweighs the effects from low quantum yields of SWCNTs. One of the technical drawbacks is the fact that not all facilities have detectors that are sensitive in the 
nIR range. Si-based cameras (which is the most common type for a charge-coupled device $(C C D))$ are not sensitive at wavelengths above $1000 \mathrm{~nm}$. Instead, near-infrared cameras employ such materials as indium-gallium-arsenide (InGaAs) for the range of $900-1800 \mathrm{~nm}$ or mercury-cadmium-telluride ( $\mathrm{HgCdTe})$ sensors for the wavelengths above $1400 \mathrm{~nm}$ [60]. But switching to another detector material is a small price to pay for bioimaging with almost no background. For example, by using this emission regime it was possible to visualize blood vessels in small animals without surgical invasion [210]. After a functionalization of SWCNTs with biocompatible surface coating and injecting it into the bloodstream of tumor-bearing mice, a detailed map of blood vessels could be clearly observed through the skin.

From 1990 to 2013, the yearly number of publications describing nIR fluorophores has increased from almost zero to 400 in case of nIR fluorphores and to 200 in case of fluorescent probes [211]. At the same time, there is only one nIR fluorescent molecule, a fluorophore based on indocyanine green, that is approved by the US Food and Drug Administration (FDA) for clinical applications [212]. Another example is an advancing class of cyanine fluorophores with emission in the nIR-I range $\left(\lambda_{e x} \sim 820 \mathrm{~nm}, \lambda_{e m} \sim 850 \mathrm{~nm}\right)$, high quantum yields $(>0.50)$ and fluorescence lifetimes in nanoseconds range [213]. Some of these cyanine-based nIR fluorophores have been conjugated to RGD-peptides to monitor their uptake in $\alpha_{5} \beta_{1}$ integrin positive tumors [214]. Other promising nIR fluorophores in the nIR-II include quantum dots (QD) and plasmonic nanoparticles, e.g. silver or gold nanoparticles [60]. In contrast to SWCNTs, their optical properties can be tuned by their size and shape. However, QDs often contain toxic metal particles, such as $\mathrm{PbS}$ or $\mathrm{HgTe}$, which hinders their biomedical application. Therefore, the library of nIR fluorophores is very limited. 


\subsubsection{Egyptian Blue as nIR emitter}

So far, there was one novel nIR fluorophore overseen in most publications: Egyptian Blue $\left(\lambda_{e x}\right.$ $=625 \mathrm{~nm}, \lambda_{e m}=930 \mathrm{~nm}$, Fig. 15a). The absorption and the color properties of this pigment are well-known, but the emission in the near-infrared was only recently described by Accorsi et al. 215. The nIR emission first became useful when one had to investigate artifacts where the handling should be kept to a minimum. With the conversion of visible light into the nIR emission, Egyptian Blue is ideal for non-invasive investigations. It was used to detect traces of Egyptian Blue in old monuments, such as the Greek Parthenon [216]. The longevity of the pigment itself and its PL is evident. Ancient decorations have survived for several thousand years in both dry and damp surroundings, as well as in various museums, and still exhibit bright luminescence without photobleaching. An example of the nIR photoluminescence collected from an antique sample can be seen in Figure 15b. Aside from the nIR emission, the Stock-shift of $\approx 300 \mathrm{~nm}$, PL lifetimes in $\mu$ s-range, its quantum yield is the highest for all known nIR fluorophores: QY (Egyptian Blue $)=10.5 \%, \mathrm{QY}(\operatorname{ssDNA} / \mathrm{SWCNTs}) \sim 0.1$ $1 \%$ 215], 96 .

\section{Geometric structure of Egyptian Blue}

Egyptian Blue $\left(\mathrm{CaCuSi}_{4} \mathrm{O}_{10}\right)$ is a blue pigment known since $3600 \mathrm{BC}$ [217]. It was the earliest artificial pigment that was synthesized by mankind. It was widely used in murals and decoration, and its application spread out to the Mediterranean areas, Mesopotamia and the ancient Roman Empire. With the fall of the Roman Empire, the knowledge of its synthesis was lost. Despite its rich history, Egyptian Blue is also a defined compound with the chemical formula: $\mathrm{CaCuSi}_{4} \mathrm{O}_{10}$ (Fig. 15a) 218. It can be synthesized by mixing the following minerals together: lime $\left(\mathrm{CaCO}_{3}\right)$, sand $\left(\mathrm{SiO}_{2}\right)$, a mineral containing copper (e.g. malachite $\left(\mathrm{CuO}_{2}\left(\mathrm{COO}_{3}\right)(\mathrm{OH}) \mathrm{O}_{2}\right.$ or metallic copper), as well as a small amount of alkaline base, such as potassium carbonate $\left(\mathrm{K}_{2} \mathrm{CO}_{3}\right)$ or soda $\left(\mathrm{Na}_{2} \mathrm{CO}_{3}\right)$. The mixture is then heated up to temperatures around $800-900^{\circ} \mathrm{C}$ [219]. It is important that the mixture is exposed to oxygen to prevent the formation of red cuprite $\left(\mathrm{Cu}_{2} \mathrm{O}\right)$.

$$
\mathrm{CuO}_{2}\left(\mathrm{COO}_{3}\right)(\mathrm{OH}) \mathrm{O}_{2}+8 \mathrm{SiO}_{2}+\mathrm{CaCO}_{3} \underset{800-900^{\circ} \mathrm{C}}{\stackrel{\text { alkaline base }}{\longrightarrow}} 2 \mathrm{CaCuSi}_{4} \mathrm{O}_{10}+3 \mathrm{CO}_{2}+\mathrm{H}_{2} \mathrm{O}
$$

Egyptian Blue is a copper silicate of the cuprorivaite class $\mathrm{MCuSi}_{4} \mathrm{O}_{10}$, where $\mathrm{M}$ is an alkaline earth metal $(\mathrm{M}=\mathrm{Ca}, \mathrm{Sr}, \mathrm{Ba})$. Two alkaline earth metals Calcium and Barium result in two blue pigments: the aforementioned Egyptian Blue $\left(\mathrm{CaCuSi}_{4} \mathrm{O}_{10}\right)$ and the Han Blue $\left(\mathrm{BaCuSi}_{4} \mathrm{O}_{10}\right)$. Latter was synthesized during the Han dynasty (208 B.C.) in China 220]. Both compounds are isostructural, have the same $\mathrm{Cu}-\mathrm{O}$ distances and space group (P4/ncc) 
221]. A similar compound with two less equivalents of quartz than Han Blue is Han Purple $\left(\mathrm{BaCuSi}_{4} \mathrm{O}_{6}\right)$. The crystal structure of $\mathrm{MCuSi}_{4} \mathrm{O}_{10}$ is tetragonal with $\mathrm{SiO}_{4}$ silicate pyramids forming the structural framework. Four of those silicate pyramids form a $\mathrm{Si}_{8} \mathrm{O}_{20}$ sheet, which is responsible for the major percentage of the height of one unit cell [222]. The heart of the complex is the square-planar $\mathrm{CuO}_{4}^{6-}$ chromophore. In the complex the copper ion has a $D_{4 h}$ symmetry and is centered between four oxygen atoms in a very stable coordination. The fluorescence originates from $\mathrm{Cu}^{2+}$ ions linked by the corners of 4 tetrahedral silicate moieties $\left(\mathrm{SiO}_{4}\right)$ into a three-dimensional crystal structure. The $\mathrm{CuSi}_{4} \mathrm{O}_{10}$ layers alternate with $\mathrm{Ca}^{2+}$ or other alkaline earth metals. The stability of the structure does not allow to remove the copper ion by light exposure, high temperature, acids, etc. 215.
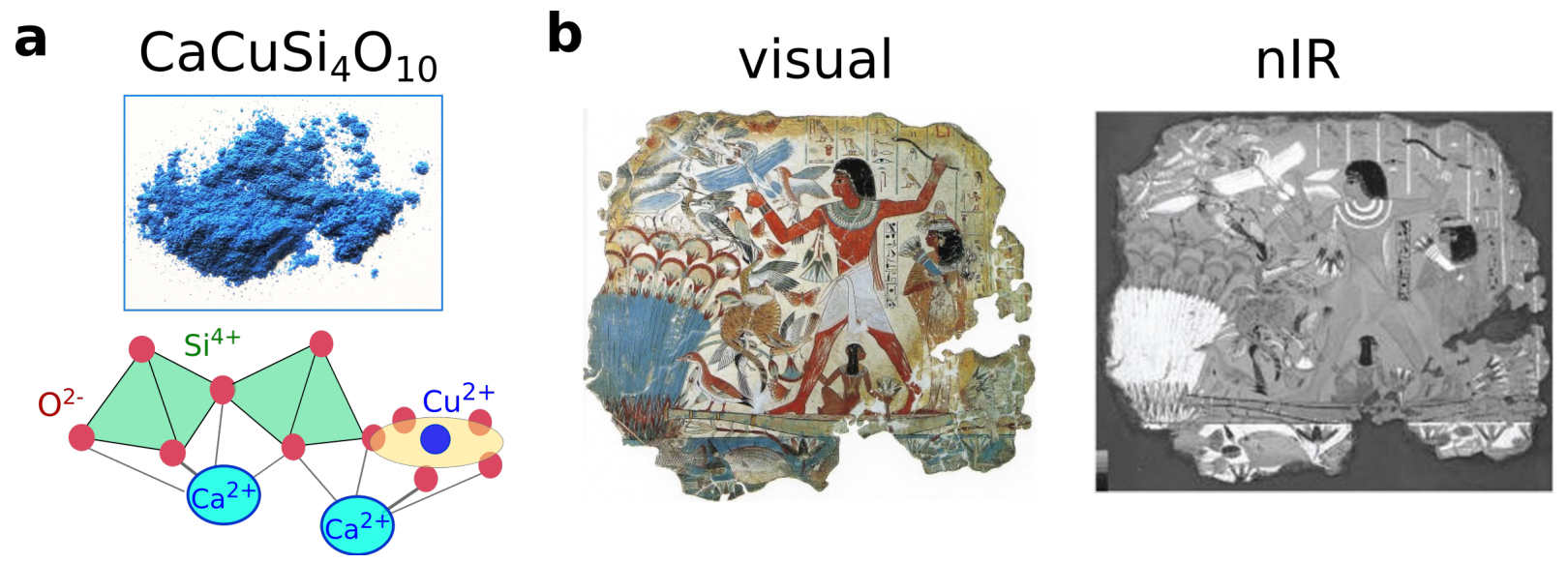

Figure 15: Optical and geometric properties of Egyptian Blue. (a) Photography image of the Egyptian Blue powder and the schematics of its unit cell with $\mathrm{Cu}^{2+}$ ions in blue, $\mathrm{Ca}^{2+}$ in turquoise, $\mathrm{O}^{2-}$ in red, and $\mathrm{SiO}_{4}^{4-}$ tetrahedra in green, (b) Hunting in the marshes, mural fragment from the tomb chapel of Nebamun (1400-1300 BC). Visible (top) vs. nIR photoluminescence (bottom) photography. White areas in the second image correspond to Egyptian Blue pigment. Adapted with permission from [215], all rights lie by The Trustees of the British Museum, British Museum, London, UK.

On the contrary, the alkaline earth metals act as independent counterions and are positioned between the layers. Due to its layered structure, Egyptian Blue easily forms multilayer nanosheets [223]. The structure of a unit cell is shown in Figure 15b. The nature of alkaline earth metals has a direct influence on the luminescence of $\mathrm{Cu}^{2+}$. The ion radius of alkaline earth metals are decreasing as following: $\mathrm{Ba}^{2+}>\mathrm{Sr}^{2+}>\mathrm{Ca}^{2+}$. The same gradual trend can be observed in the red-shift of the emission maxima (930, 936, and $964 \mathrm{~nm}$, respectively) [224]. As the ion radius gets larger, the lattice expanses and the crystal field experienced by $\mathrm{Cu}^{2+}$ gets weaker. Thus, the lattice expansion of the unit cell results in a red-shift both in absorption and in emission spectra 221]. 


\section{Photophysics of Egyptian Blue}

The optical properties of transition-metals are generally determined by (1) the nature of the ligands, the metal cation and its coordination number, (2) the geometrical arrangement of these ligands around the metal cation, and (3) the distances between the metal cation and the ligands [216]. To understand the photophysical properties of Egyptian Blue it is helpful to reconstruct the energy diagram of $\mathrm{Cu}^{2+}$ under consideration of these influences (Fig. 16). We start with a free $\mathrm{Cu}^{2+}$ ion with the outer electron configuration of $3 \mathrm{~d}^{9}$. First, it translates into the octahedral ligand field of a $\mathrm{CuO}_{4}^{6-}$ complex, accompanied by splitting of $d^{9}$ into 5 energy levels $\left(e_{g}\right.$ and $t_{2 g}$ ). The higher levels consists of two orbitals: $x^{2}-y^{2}$ and $z^{2}$. The three orbitals with lower energies are $x y, x z$ and $y z$. The next transition is into a square-planar $\mathrm{CuO}_{4}^{6-}$ complex with lower symmetry $O_{h} \longrightarrow D_{4 h}$. Here, the $x^{2}-y^{2}$ and the $z^{2}$ orbitals experience two different influences due to the Jahn-Teller effect and the lattice constraint. The ligands are positioned in the plane around copper, thus making the $x^{2}-y^{2}$ orbital the most unfavorable. Its energy is now the highest. At the same time, the $z$-direction is no longer energetically unfavorable, and the energy of the $z^{2}$ orbital is now the lowest. From the 3 degenerated $t_{2 g}$ orbitals, the $x y$-orbital has a slightly higher energy than $x z$ and $y z$. In the final step, the $\mathrm{CuO}_{4}^{6-}$ unit is embedded into the $\mathrm{CaCuSi}_{4} \mathrm{O}_{10}$ structure. Here, the z-direction gets slightly less favorable, as the $\mathrm{Cu}^{2+}$ ion experiences ligands and counterions from the the various layers of the whole complex.

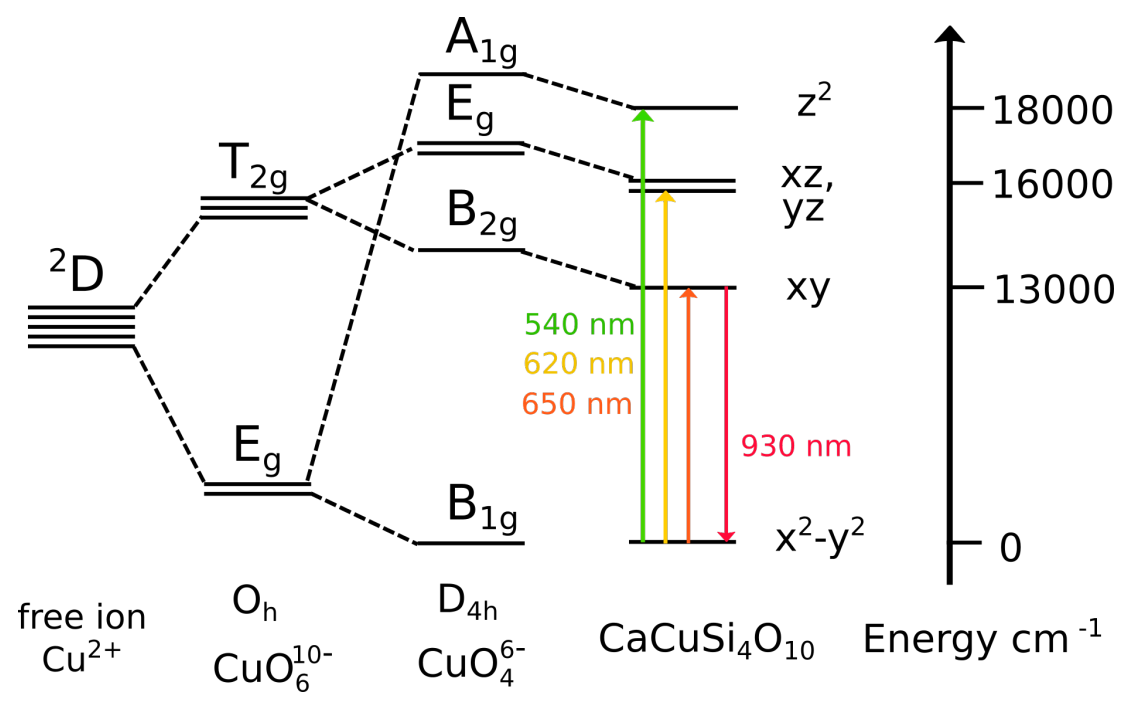

Figure 16: Optical properties of $\mathbf{C u}^{2+}$ in Egyptian Blue. The energy diagram for the conversion from the free $\mathrm{Cu}^{2+}$ ions into the $\mathrm{CaCuSi}_{4} \mathrm{O}_{10}$. 
The excitation spectrum of Egyptian Blue covers a wide range of $500-700 \mathrm{~nm}$, which matches the three optical transitions from the ground state ${ }^{2} B_{1 g}$ into ${ }^{2} B_{2 g},{ }^{2} E_{g}$ and ${ }^{2} A_{1 g}$ (650, 620, and $540 \mathrm{~nm}$, respectively) [221]. There is a difference of $-0.4 \mathrm{eV}$ between the highest absorption energy of Egyptian Blue $(540 \mathrm{~nm}=2.3 \mathrm{eV})$ and the highest absorption energy of the $\mathrm{CuO}_{4}^{6-}$ complex $(460 \mathrm{~nm}=2.7 \mathrm{eV})$. This difference is attributed to the anisotropic $E_{R}(r)$ field which is created by the whole crystal and affects all electrons in the complex. The anisotropic $E_{R}(r)$ field of the Egyptian Blue does not occur in the more symmetrical $\mathrm{CuO}_{4}^{6-}$ complexes [216]. Thus, not all $\mathrm{CuO}_{4}^{6-}$ exhibit bright blue color, which results from the lack of absorption in the blue region in case of $\mathrm{CaCuSi}_{4} \mathrm{O}_{10}$.

The emission of $\mathrm{CaCuSi}_{4} \mathrm{O}_{10}$ is centered around $930 \mathrm{~nm}$ and results from the energy transition from the lowest excited state to the ground state ${ }^{2} B_{2 g}(x y) \longrightarrow{ }^{2} B_{1 g}\left(x^{2}-y^{2}\right)[216]$. The emission should be formally the reverse of the corresponding absorption transition. Yet the energies differ:

$$
\begin{gathered}
\text { Emission: }{ }^{2} B_{2 g} \longrightarrow{ }^{2} B_{1 g}=10500 \mathrm{~cm}^{-1} \\
\text { Absorption: }{ }^{2} B_{1 g} \longrightarrow{ }^{2} B_{2 g}=12500 \mathrm{~cm}^{-1}
\end{gathered}
$$

When one considers only the unit cell and the local environment of $\mathrm{Cu}^{2+}$ ions, Egyptian Blue has an inversion center. Thus, the intraconfigurational electronic (d-d) transitions are parity-forbidden. The observed emission intensity can be measured due to the unsymmetrical vibrational modes coupled with the electronic transitions, which disrupts the symmetry [225]. Non-radiative relaxation from the highest energy level ${ }^{2} A_{1 g}$ to ${ }^{2} B_{2 g}$ is efficient. The relaxation from ${ }^{2} B_{2 g}$ to the ground level ${ }^{2} B_{1 g}$ is less populated and thus less frequent 225]. Therefore, the emission exhibits very long luminescence decay times. The reported lifetimes range from 107 to $130 \mu \mathrm{s}$ 215, 226 and was calculated to $\tau=124 \mu \mathrm{s}$ in our measurements (see Sec. 4.3 .2 for details). 


\section{Experimental Procedures}

\subsection{Materials}

\subsubsection{Chemicals}

Unless otherwise noted, all chemicals were purchased from Sigma Aldrich (Taufkirchen, Germany). 6,5-chirality enriched SWCNTs (Product No.: 773735), phosphate buffered saline buffer (0.01mM, pH 7.4), fibrinogen from human plasma, anti-Integrin $\alpha \mathrm{II} b \beta 3$ antibody (CD41 Antigen, anti-human, produced in rabbit), polyethylene glycol sorbitan monolaurate (Tween 20), bovine serum albumin, O-phenylenediamine dihydrochloride, polyacrylic acid (average Mv 450,000), polyallylamine hydrochloride (average Mw 58,000). 1,2dimyristoyl-sn-glycero-3-phosphoethanolamine-N [methoxy (polyethylene glycol)-1500] and 1,2-dimyristoyl-sn-glycero-3-phosphoethanolamine-N-[methoxy (polyethylene glycol)-5000] (PL-PEG1.5 and PL-PEG5k) were purchased from Avanti Polar Lipids (Germany). Succinimidyl 4-(N-maleimidomethyl)cyclohexane-1-carboxylate (SMCC) was purchased from ThermoFisher scientific (Germany). $\alpha \mathrm{II} b \beta 3$ integrin was provided by Joachim Spatz (MPI, Medical Research). Goat Anti-Rabbit IgG with horseradish peroxidase was purchased from Pharmingen, BD Bioscience Europe. Egyptian Blue was purchased from the Kremer Pigment (Germany).

\subsubsection{Oligonucleotides}

Unmodified sequences included $(\mathrm{T})_{30},(\mathrm{GT})_{15},(\mathrm{AT})_{15}$ and $(\mathrm{G} 3 \mathrm{~T})_{7}$. Amino-modified ssDNA oligonucleotides $(\mathrm{GT})_{n}$ and $(\mathrm{C})_{n}$ sequences included $\left((\mathrm{GT})_{15^{-}} \mathrm{C} 3\right.$ linker- $\mathrm{NH}_{2},(\mathrm{GT})_{10^{-}}$ C3linker- $\mathrm{NH}_{2},(\mathrm{C})_{30}-\mathrm{C} 3$ linker- $\mathrm{NH}_{2}$ and $(\mathrm{C})_{20}-\mathrm{C} 3$ linker- $\mathrm{NH}_{2}$. All oligonucleotides was provided with standard desalting and stored at $-20^{\circ} \mathrm{C}$. Before preparation, each batch was thawed and hydrated with MilliQ deionized water.

\subsubsection{Cell culture}

Madin-Darby canine kidney cells, strain II (MDCK II; Health Protection Agency, Salisbury, UK) were cultured in minimum essential medium (MEM) with Earle's salts, $2.2 \mathrm{~g} / \mathrm{L}$ NaHCO3, 10\% fetal bovine serum, and $4 \mathrm{mM}$ L-glutamine in a humidified incubator set to $37^{\circ} \mathrm{C}$ and $5 \% \mathrm{CO}$. Cells were released using trypsin/EDTA (0.05:0.02\%) and subcultured three times per week. Prior to experiments, MaTek glass bottom Microwell dishes (35 mm petri dish, 14 microwell, 1.5 coverglass, Nr.: P35G-1.514-C) were coated overnight with various DNA-peptide/SWCNTs and washed before the experiments. 125,000 cells were 
seeded on freshly prepared MaTek dishes and incubated at $37^{\circ} \mathrm{C}$ and $7.5 \% \mathrm{CO}_{2}$ in a humidity chamber (Thermo Fisher Scientific, Waltham, MA). MDCKII cells were kindly provided by Angela Rübeling (WG Prof. Janshoff, University Göttingen). Platelets rich plasma was provided by the group of Luise Erpenbeck/Prof. Schön (University Medical Center, Göttingen). Human blood was layered 1:1 on top of a histopaque 1119 (sigma) gradient and centrifuged for $21 \mathrm{~min}$ at $2100 \mathrm{~g}$. Platelets rich plasma was collected from the opaque layer on top of the histopaque 1119 gradient.

\subsection{Procedures and synthesis}

\subsubsection{Polymer/SWCNT dispersion}

Depending on the experiment, various ssDNA-solutions suspended in $0.1 \mathrm{M} \mathrm{NaCl}$ were added to SWCNT powder in phosphate-buffered saline (PBS). Samples were sonicated with a 3 mm probe tip (Fisher Scientific, Model 120 Sonic Dismembrator, 20\% amplitude) for 10 min, followed by centrifugation for 120 min (Eppendorf Centrifuge 5415 D) at $13.200 \mathrm{rpm}$ to remove large bundles or aggregates. The supernatant was collected and the pellet discarded. For suspensions with polymer materials other than ssDNA (polyacrylic acid, lipids, ect.), samples were sonicated for two hours.

\subsubsection{Peptide synthesis}

Linear (CVRGDF, CVRGEF) and bridging (CVRGDFC) peptides were synthesized via solid phase peptide synthesis with Fmoc-protected amino acids. Before the synthesis, rink amide 4-(2',4'-Dimethoxyphenyl-Fmoc-aminomethyl)-phenoxyacetamido-norleucylMBHA resin (100-200 mesh, Novabiochem) was dissolved in dimethylformamide (DMF), transferred into the reaction vessel and shaken for 1 hour. In the first synthesis step, resin was shaken with piperidine / DMF (3:7) 3x for 10 min to achieve cleavage of the fluorenylmethyloxycarbonyl (Fmoc) protecting group and rinsed with DMF after each deprotection step. To remove residual piperidine from the resin and the reaction vessel the resin was washed with DMF (2x), shaken in DMF for 3 min and rinsed again in DMF (3x). For coupling 4 eq of the N-terminal protected amino acid, 3.6 eq of the coupling reagent 2-(1H-Benzotriazole1-yl)-1,1,3,3-tetramethyluronium (HBTU) and 6 eq of N,N-Diisopropylethylamine (DIEA) were dissolved in DMF and added into the reaction vessel. The reaction was allowed to take place for $30 \mathrm{~min}$ for the first 5 amino acids and 1 hour for longer peptides to atone for steric hindrance. The coupling of the first amino acid as well as the coupling of Arg was done twice, in the first case because of the close distance to the resin, and in the latter because of steric hindrance. After coupling, the resin was washed with DMF (3x) and checked with Kaiser 
test for free primary amine groups. From each of the following solutions, one drop was added to a few resin beads: $5 \mathrm{~g}$ ninhydrin in $100 \mathrm{ml}$ ethanol, $80 \mathrm{~g}$ phenol in $20 \mathrm{ml}$ ethanol, $2 \mathrm{ml}$ of an aqueous $0,001 \mathrm{M}$ potassium cyanide solution in $98 \mathrm{ml}$ pyridine. The mixture was heated up to $110^{\circ} \mathrm{C}$ for one minute. In case of blue coloration the coupling reaction was repeated to achieve better yields. If the Kaiser test was negative (yellow solution), the amino acid on the resin was deprotected (piperidine/DMF $3: 7,3 \mathrm{x}$ for $10 \mathrm{~min}$ ), rinsed with DMF (3x for $10 \mathrm{~min}$ ) and the next amino acid was added. To cleave the amino-acid from the resin, the resin was dried with dichloromethane (DCM) and ether under air, and then kept overnight under vacuum in a drying chamber. One third of the following solution was then added to the dry resin at a time: $47.25 \mathrm{~mL}$ trifluoro acetic acid (TFA), $1.25 \mathrm{~mL}$ water, $1.25 \mathrm{~mL} \mathrm{1,2-}$ ethandithiol (EDT) and $0.5 \mathrm{~mL}$ triisopropylsilane (TIS). The solution was shaken with resin $2 \mathrm{x}$ for $30 \mathrm{~min}$ and $1 \mathrm{x}$ for $60 \mathrm{~min}$. After shaking and rinsing all three parts were collected in a round bottom flask. Afterwards, the cleavage solution was removed by a rotary evaporator at 120 mbar and $50^{\circ} \mathrm{C}$. Ice-cold diethylether was then added drop wise to the concentrated solution until a stable precipitate had formed. Then, another 30-40 mL ice-cold diethylether was added. The precipitate was collected by suction and filtration. After dissolving it in RP-A (99\% water, 1\% Acetonitrile, 0.1\% TFA) it was isolated by freeze drying overnight. All of the synthesized peptides were characterized by ESI-MS analysis (Apex IV, Bruker Daltonics, Billerica, MA, USA).

\subsubsection{Conjugation of SMCC to DNA oligonucleotides}

Following the protocol by Williams and Chaput [227], oligonucleotides were diluted in PBS buffer. In a separate tube succinimidyl 4-(N-maleimidomethyl)cyclohexane-1-carboxylate (SMCC) was diluted in acetonitril to the final concentration of 1:10 with respect to DNA. After mixing together, the resulting solution was vortexed vigorously for 30 seconds and left at RT for 1h. Afterwards, the excess of SMCC was separated from the SMCC-oligonucleotides via ethanol extraction. The SMCC-oligonucleotides were then functionalized to nanotubes. For this, SMCC-oligonucleotides were solubilized and added to $0.5 \mathrm{mg}$ of 6,5-chirality enriched SWCNT in $1.5 \mathrm{~mL}$ phosphate-buffered saline (PBS). The resulting suspension was first tip sonicated for $10 \mathrm{~min}$ (Fisher Scientific Model 120 Sonic Dismembrator, 20\% amplitude) and subsequently centrifuged at 16,100 g (2×30 min). Any excess of SMCC-oligonucleotides was removed during spin filtration (Vivaspin 500, 100,000 MWCO, Satorius). Afterwards, the peptide solution (1:1 with DNA) was added, vortexed for 30 seconds and left to react at RT overnight. Again, the excess of peptide was removed via spin filtration. In case of bridged DNA-linker-peptide-linker-DNA constructs, the conjugation of SMCC-oligonucleotides to peptide containing cysteines on both ends was achieved by adding 2 equivalents of SMCColigonucleotides to the peptide solution, vortexing vigorously for 30 seconds and letting the 
solution to react at RT overnight. Afterwards, the excess of peptide was removed via spin filtration.

\subsubsection{ELISA Essay}

Binding of integrin was detected by specific antibodies in an enzyme-linked immunosorbent assay (ELISA). Every experiment was performed in duplicates and the resulting inhibition curves were analyzed using OriginPro 8 software to determine IC50 values. 96-well ELISA plates (Nunc-Immuno MicroWell 96-Well Plates, Thermo Scientific, VWR) were coated over night at $4^{\circ} \mathrm{C}$ with fibrinogen $(20 \mathrm{mg} / \mathrm{mL})$ in PBS Buffer $(0.01 \mathrm{mM}, \mathrm{pH} 7.4)$. Plates were subsequently washed three times with PBST buffer (PBS buffer $+0.01 \%$ Tween 20, pH 7.4) and blocked for one hour at room temperature 1\% BSA buffer (1\% BSA in PBS buffer). Solubilized integrin $\alpha \operatorname{Ib} \beta 3(200 \mathrm{nM})$ and a serial dilution of integrin inhibitor (RGD containing DNA-peptide/SWCNTs) were incubated in coated wells for $1 \mathrm{~h}$ at room temperature. The plate was then washed three times with PBST buffer and $100 \mu \mathrm{L} /$ well of primary antibody (CD41, 1:500 dilution in PBS) were incubated for $1 \mathrm{~h}$ at room temperature. After washing three times with PBST, $100 \mu \mathrm{L} /$ well of the secondary anti-body (anti-rabbit HRP-conjugate, 1:1000 dilution in PBS) were applied for $1 \mathrm{~h}$ at room temperature. After this treatment the plate was washed three times and the binding visualized using O-phenylenediamine dihydrochloride (OPD, $0.4 \mathrm{mg} / \mathrm{ml}, 200 \mu \mathrm{L}$, in $0.05 \mathrm{M}$ citrus acid buffer $(\mathrm{pH}=5.0)$ ) as substrate for horseradish peroxidase and incubated for $30 \mathrm{~min}$ in the dark. Afterwards, $1 \mathrm{M}$ HCL (50 $\mu \mathrm{L})$ was added to stop the reaction. The color reaction was measured at $492 \mathrm{~nm}$ with a plate reader Anthos 2010 Photometer (Microsysteme GmbH, Germany).

\subsection{Analytical techniques}

\subsubsection{UV-Vis-nIR spectroscopy}

Absorption values for DNA concentrations were measured via Nanodrop peqlab 2000c. Absorption spectra for various polymer/SWCNTs constructs were collected with JASCO V-670 spectrophotometer (Spectra Manager Software) using a $10 \mathrm{~mm}$-path cuvette. ELISA data were recorded using Anthos 2010 Photometer (Microsysteme GmbH, Germany).

\subsection{2 nIR spectroscopy and imaging}

Near-infrared emission spectra were collected on a Shamrock 193i spectrograph (Andor Technology Ltd., Belfast, Northern Ireland) coupled to an Olympus BX53 microscope using an 
exposure time of $5 \mathrm{~s}$, slit width of $500 \mu \mathrm{m}$ and an Andor iDus InGaAs 491 array NIR detector. SWCNTs were excited at $560 \mathrm{~nm}$ with power up to $500 \mathrm{~mW}$. All fluorescence signals were analyzed with OriginPro 8 and MATLAB R2008a. NIR images were collected with a Xenics nIR camera (Xeva-2.5-320).

\subsubsection{Raman spectroscopy}

The experiments were carried out using a Horiba Jobin Yvon HR800 UV Raman spectrometer $(\lambda=785 \mathrm{~nm}, 500 \mathrm{~mW}$, Xtra High-Power Single-Mode Laser) equipped with a 10x wide-field objective, a $500 \mathrm{~nm}$ confocal hole and a CCD detector. Each spectrum was collected with 3 iterations and 10 seconds measurement time per segment (approx. $200 \mathrm{~nm}$ ). All Raman spectra were recorded using LabSpec and corrected using an internal correction (ICS, Horiba).

\subsubsection{AFM spectroscopy}

AFM samples were prepared by dropping Egyptian Blue samples onto a silica wafer and vaporizing the aqueous phase. Afterwards, the topological data was collected with MFP3DTM (Asylum Research, Santa Barbara, CA, USA) using air tapping mode (dynamic) with MLCT cantilevers (Bruker, Camarillo, CA, USA). Prior to each experiment, the spring constant of the cantilever was determined on a flat silica wafer. Analysis of the acquired images was performed via the open-source software Gwyddion. 


\section{Results and Discussions}

\subsection{Investigation of the interaction between redox active analytes and polmyer/SWCNTs}

Results presented in this chapter have been published in:

Elena Polo and Sebastian Kruss,

Impact of Redox-Active Molecules on the Fluorescence of Polymer-Wrapped Carbon

Nanotubes,

J. Phys. Chem. C, 2016, 120(5), pp 3061-3070

DOI: $10.1021 /$ acs.jpcc.5b12183

Data and figures are reproduced or adapted from [55] with permission from American Chemical Society Publication.

The fluorescence of SWCNTs is excitonic rather than electronic in nature. After the excitation, excitons move along the surface of SWCNTs and can be affected by smallest changes in their local environment [114]. Therefore, bionanosensors based on SWCNTs are sensitive down to the detection of single molecules [64], [57]. However, the exact sensing mechanism behind the fluorescence change is not fully understood.

This chapter will start with a short overview over possible sensing mechanisms. We designed our experiments to either support or dismiss each possibility. Then we will discuss interfering molecules and which of their chemical residues or properties (e.g. redox potential) are relevant to our investigation. Based on this, we will create a library of promising analytes to investigate possible trends or special molecule features. Then we will investigate how small redox-active molecules affect the fluorescence of polymer/SWCNTs, while systematically changing both the analytes and the polymer phase around SWCNTs. In the final section, we will review the results of our experiments and its implication on the potential sensing mechanism.

\subsubsection{Mechanisms for sensing in SWCNT-based sensors}

So far, a variety of causes was considered to influence SWCNT fluorescence: redox reactions, availability of free surface area around the SWCNT, conformational changes of the organic phase or other changes of exciton diffusion pathways induced upon binding of another molecule [56], [228], [229]. It is also possible that the sensing mechanism is contributed by several of these pathways, and the priority of each pathways vary from analyte to analyte. 
Another interesting finding showed that while all ssDNA sequences react to catecholamines in a similar way, cytosine-containing sequences exhibit both the highest starting PL intensity and the lowest responses [56]. This slight change in the organic phase suggests that not only the analyte but also the exact nature of the organic phase contributes to the PL response. Using only one polymer wrapping is a common problem in the most polymer/SWCNT studies. The underlying assumption that small changes of the polymer (e.g. different ssDNA sequences or various lengths) are negligible underestimates the influence of organic phases on the sensing mechanism. Therefore, we will use a wider polymer library to better characterize the sensing mechanism of polymer/SWCNTs.

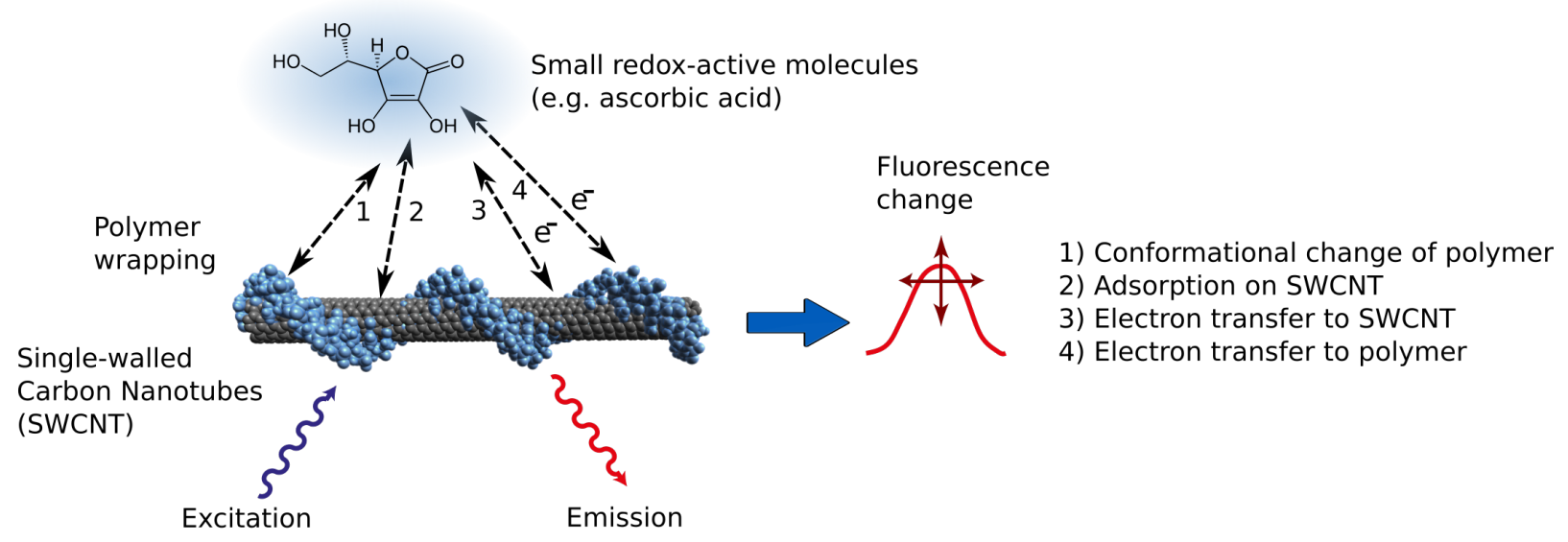

Figure 17: Schematic of a polymer/SWCNT sensor. Illustration of possible interaction routes between redox-active molecules (e.g. ascorbic acid) and polymer wrapped SWCNTs (polymer/SWCNTs). Adapted with permission from own work [55].

There are four possible pathways how an analyte could affect the initial polymer/SWCNTs fluorescence (Fig. 17):

- The analyte directly adsorbs onto the free SWCNT surface. Fluorescence change is based on the modified organic phase of the SWCNT or possible adsorption/interactions between the analyte and the naked SWCNT.

- The analyte interacts with the polymer phase. This scenario leads to conformational changes of polymers around SWCNT that might affect exciton decay routes.

- A redox-active analyte could reduce or oxidize the SWCNT.

- Alternatively, a redox-active analyte could reduce or oxidize not the SWCNT itself but the surrounding organic polymer phase. 


\section{Library of analytes to test}

In a biological environment, interference with other compounds plays a crucial role in sensor development. For dopamine sensing one of such molecules is ascorbic acid. It is an important antioxidant that protects our cells against oxidative stress damage and participates in the neuronal metabolism interface [230]. Its concentration in the brain exceeds that of almost all other organs (micromolar range) and is known to interfere with SWCNT-based or electrochemical dopamine detection [231. The results of screening experiments showed that the sensor response to a chosen analyte is additive regarding interfering molecules [54]. This means that introducing ascorbic acid into the system would already increase the overall PL intensity of SWCNTs, and therefore, the sensor response would be a lot smaller. This result supports the idea that either an interfering substance or an analyte can both occupy a binding site on the ssDNA/SWCNT surface and cause a PL change. Other possible interfering molecules might have similar chemical structure to dopamine. Dopamine contains three important features that can be relevant for molecular recognition of ssDNA/SWCNT sensors: (1) two hydroxy groups (catechol-moiety), (2) an aromatic ring, and (3) a primary amine group. Indeed, most ssDNA/SWCNT sensors have difficulties to distinguish between different catecholamines. So far, $(\mathrm{GT})_{10} / \mathrm{SWCNT}$ sensors were able to discriminate between dopamine and norepinephrine at the background of norepinephrine (50 nM). It was possible to detect dopamine in the presence of equimolar amounts of norepinephrine on a single SWCNT level [57]. However, at higher initial concentrations of interfering molecules ssDNA/SWCNTs can not discriminate between similar analytes.

Interestingly, it was also shown that the analyte molecules with one free hydroxy group induced smaller fluorescence changes than molecules with two free hydroxy groups, such as catecholamines or the ascorbic acid [56]. Instead, an amine group does not seem to be important for the sensor response. For example, serotonin, a neurotransmitter with a primary amine group, is known to decrease the PL intensity of SWCNT-based sensors [54]. As for the third criteria, aromatic and redox-active neurotransmitters generally show higher responses than non-redox-active analytes, such as glycine. Similar redox-potential can play a crucial role if the recognition mechanism is based on a redox-reaction. Some findings support this hypotheses: e.g. both L-Dopa and DOPAC produce similar sensor responses as dopamine. But all three neurotransmitters have not only comparable redox potentials, but are also very similar in their chemical structures. In contrast, uric acid has a redox potential comparable to dopamine, but a very different structure. It does not produce any intensity change when added to ssDNA/sensors [54]. At the same time, it was reported that reducing agents, such as dithiothreitol or trolox, can brighten the ssDNA/SWCNTs fluorescence up 
to 10 times 101. The intensity change was reversible upon removal of the reducing agent, the same effect as for dopamine and ssDNA/SWCNT sensors. Lee et al. suggested that the interaction between the reducing molecules and the ssDNA/SWCNT sensors must be non-covalent to be reversible. They proposed that reducing molecules would reduce the defect sites on the SWCNT sidewalls and therefore cause a brightening.

Under these considerations we selected several dopamine-related molecules, reducing compounds, and in most cases also their oxidized equivalents (Fig. 18). The library of analytes includes: ascorbic acid/oxidized ascorbic acid, nicotinamide adenine dinucleotide $(\mathrm{NAD}) /$ reduced nicotinamide adenine dinucleotide (NADH), dopamine, (-)-epinephrine, 6-hydroxy-2,5,7,8-tetramethylchroman-2-carboxylic acid (trolox), cysteine/cystin, glutathione/oxidized glutathione, and riboflavin.<smiles>NCCc1ccc(O)c(O)c1</smiles>

Dopamine<smiles>CNCC(O)c1ccc(O)c(O)c1</smiles>

(-)-Epinephrine<smiles>O=C1OC(CO)C(O)C1O</smiles>

L-Ascorbic acid<smiles>O=C1OC(C(O)CO)C(=O)C1=O</smiles>

L-Dehydroascorbic acid<smiles>CC(C)OC(=O)OCCC(NC(=O)CCC(N)O)C(=O)NCO</smiles>

Glutathione (oxidized)

$$
\text { L-Cystein }
$$<smiles>Cc1cc2nc(=O)[nH]c(=O)nc2n(C[C@H](O)[C@H](O)CO)c1=O</smiles>

Riboflavin<smiles>NC(CSSCC(N)C(=O)O)C(=O)O</smiles>

L-(-)-Cystin<smiles>NC(O)CCC(=O)NC(CS)C(=O)NCO</smiles>

Glutathione (reduced)

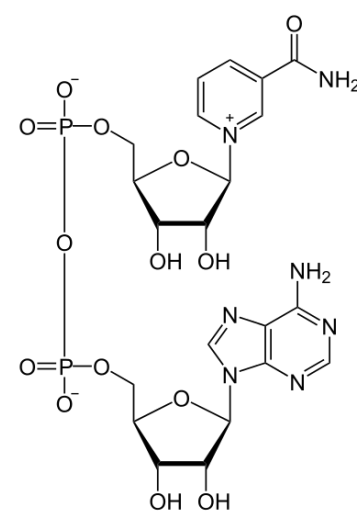

NAD

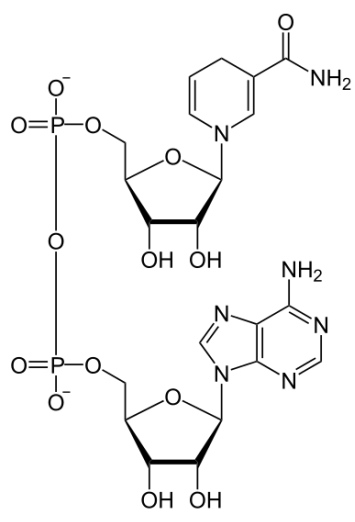

$\mathrm{NADH}$

Figure 18: Library of analytes. Figure presents a selection of small redox-active molecules. The choice of possible molecules was based on chemical similarity to dopamine and the redox potential. If possible, alongside with each reducing molecule we also used their oxidized equivalent (e.g. ascorbic acid and dehydroascorbic acid). 


\section{Various organic phases for polymer/SWCNT combinations}

Apart from testing various analytes we also want to understand how the organic phase around the SWCNTs modulates the nIR fluorescence. Typical surfactants for SWCNT functionalization are sodium dodecyl sulfate (SDS), sodium dodecylbenzene sulfonate (SDBS), and sodium cholate (SC) 232], 233]. Another very common surfactant class is ssDNA or aromatic molecules that adsorb onto the SWCNT surface via $\pi-\pi$ stacking. To achieve a stable ssDNA/SWCNT suspension sonication time of several minutes is enough. For most tensides the same result requires several hours of sonication. The prolonged sonication time itself is not a major problem, apart from the fact that it can alter or damage the surface of SWCNTs if conducted too excessive. But a general drawback of most tensides as wrapping agents lies in two facts: First, the surfactant molecules are in a dynamic equilibrium with the SWCNTs. This means they are easily removed from the SWCNT surface by filtration or dialysis which would cause the recurred aggregation and precipitation of SWCNTs [135]. Second, such surfactants need a critical micelle concentration to ensure SWCNT solubility. That implies that residues of surfactant have to be present in the solution to guarantee a stable polymer/SWCNT suspension. This could hinder some in vitro experiments, might affect or interact with the analyte, or lead to the denaturation of proteins [136]. Exchange from sodium cholate (SC) to ssDNA-dispersed SWCNT solution can be observed via shifts in the absorption spectra [137. Figure 19 shows a comparison between ssDNA/SWCNT vs. SDS- and SC/SWCNT spectra.
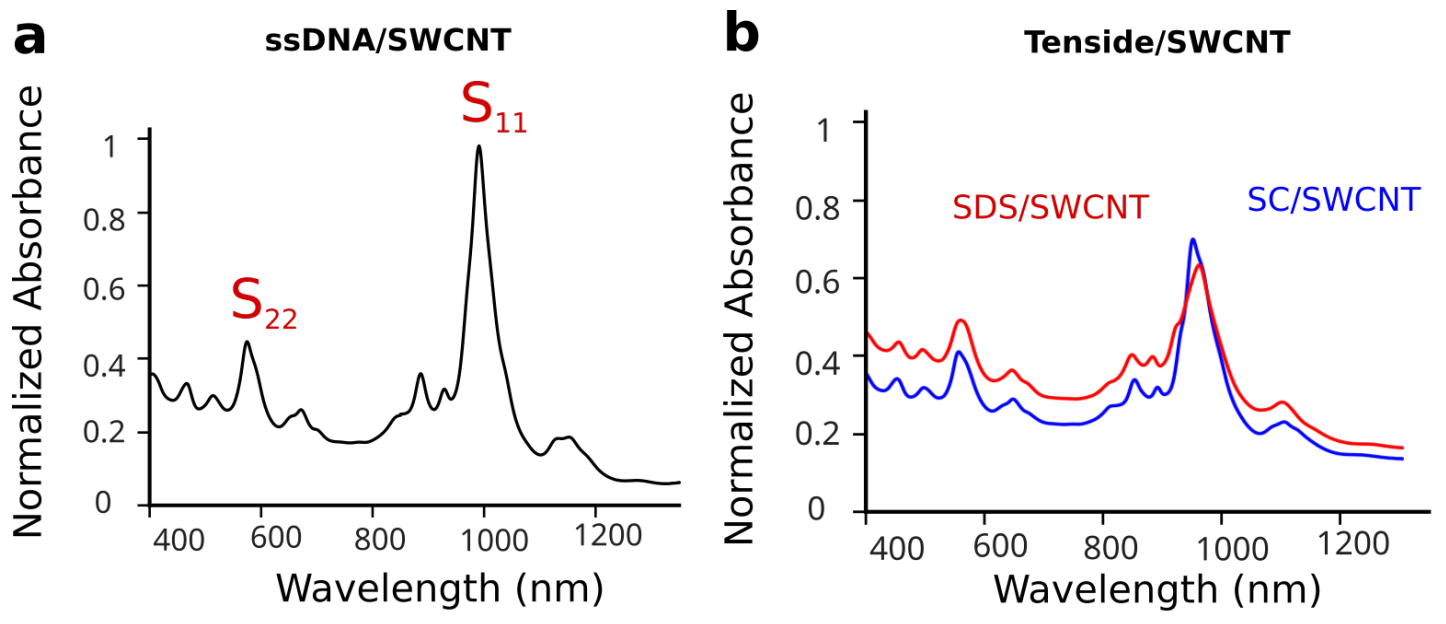

Figure 19: Absorption spectra for ssDNA- and tenside/SWCNT suspensions. (a) A typical absorption spectrum of a $(\mathrm{GT})_{15} / \mathrm{SWCNT}$ dispersion enrichted in $(6,5)$ chirality, with narrow peaks for optical transition, $\mathrm{S}_{11}$ and $\mathrm{S}_{22}$, (b) A typical absorption spectrum of SWCNTs dispersed in SDS (red) or SC (blue) polymer. 
Therefore, we used only polymers that enable stable dispersions. Figure 20 shows the selection of polymers. To represent the ssDNA class we suspended SWCNTs in four different DNA sequences: $(\mathrm{GT})_{15},(\mathrm{AT})_{15},\left(\mathrm{G}_{3} \mathrm{~T}\right)_{7}$, and $(\mathrm{T})_{30}$. DNA features phosphate groups and is negatively charged in physiological buffer. Phosphate groups might also be interesting for possible interaction with SWCNTs. On the other hand, poly(acrylic acid) (PAA) is also negatively charged in physiological buffer, but has carboxylic acid groups instead of phosphates. Polyallylamine (PAH) is positively charged in physiological buffer and should be a contrast to ssDNA and PAA. We also suspended SWCNTs in phospholipid-poly(ethylene glycol) (PL-PEG) of two different lengths (1.5k and 5k). PL-PEG has no charge and should be inert due to the long poly(ethylene glycol) chain. SWCNTs coated with PL-PEG are known as inert imaging agents because of their low immunogenicity and the ability to stay in the biological organism for prolonged periods of time [152].

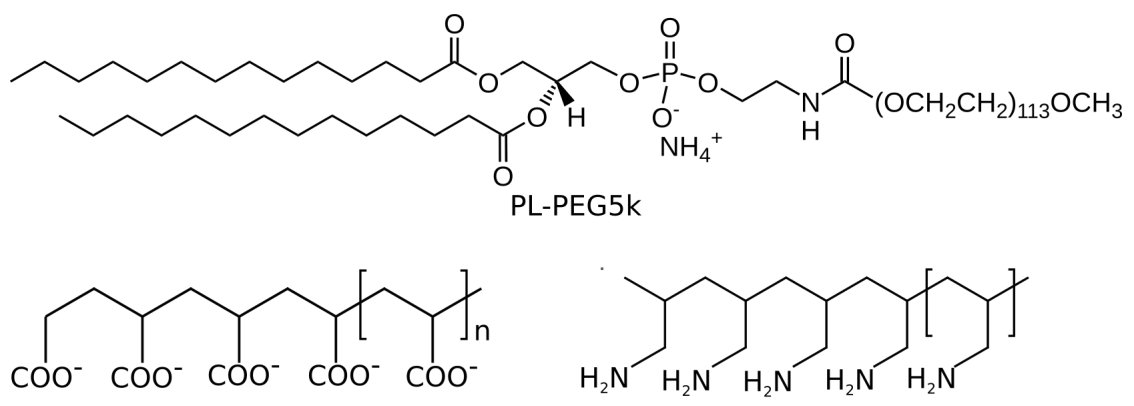

PAA (Poly(acrylic acid))

PAH (Poly(allylamine) hydrochlorid)

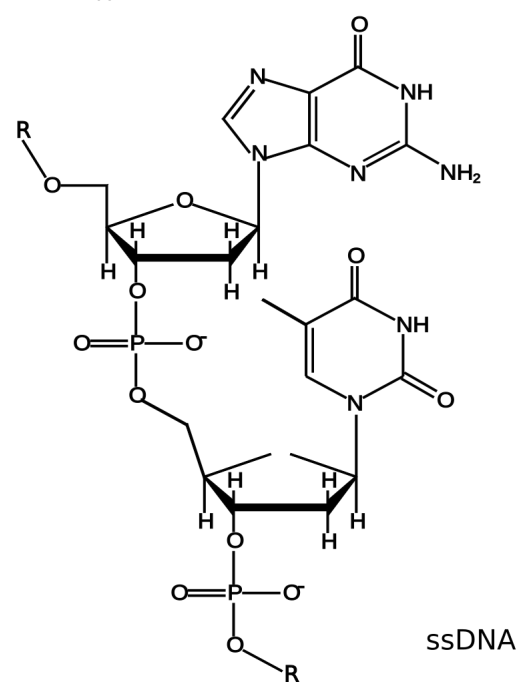

Figure 20: Library of polymers chosen as wrapping agents for polymer/SWCNT functionalization. The selection represents the neutral phospholipid-poly(ethylene glycol) (PEG), charged poly(acrylic acid) (PAA) and polyallylamine (PAH), as well as several ssDNA sequences (in experiments: $(\mathrm{GT})_{15},(\mathrm{AT})_{15},\left(\mathrm{G}_{3} \mathrm{~T}\right)_{7}$, and $\left.(\mathrm{T})_{30}\right)$. 
Typical protocols for achieving polymer/SWCNT dispersions consist of an ultrasonication step or a similar shear force treatment [234]. During this procedure, SWCNTs are individually debundled and functionalized ('wrapped') with the desired compound, followed by the centrifugation (or ultracentrifugation) and filtration steps to remove remaining aggregates and the unbound surfactant molecules. All polymer/SWCNT combinations were prepared following this protocol and characterized by UV-vis absorption spectroscopy. Absorption spectra show typical $(6,5)-\mathrm{SWCNTs}$ peaks in the nIR $\left(\mathrm{S}_{11}\right)$ and in the visible range $\left(\mathrm{S}_{22}\right)$, as shown in Figure 21). The intensity of $\mathrm{S}_{11}$ peak ensures identical SWCNT concentrations for all samples and guarantee absence of aggregation. Highly aggregated samples with bundled SWCNTs would quench the intrinsic fluorescence of a single SWCNT (s. section 2.1.3). All samples were diluted in phosphate buffered saline (PBS) at $\mathrm{pH} 7.4$ to ensure stable $\mathrm{pH}$ throughout experiments.
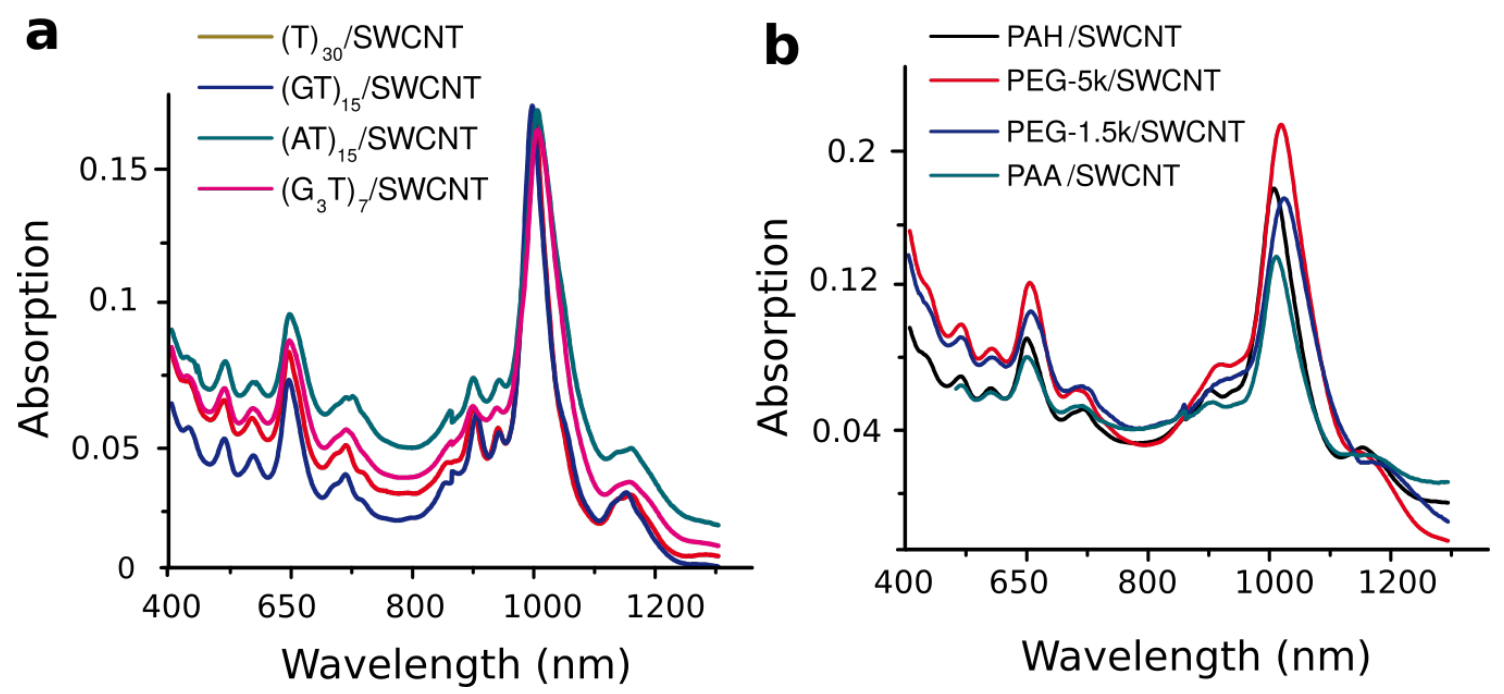

Figure 21: Absorption spectra of all polymer/SWCNTs used in the experiments. Absorption spectrum of (a) four ssDNA sequences $(\mathrm{GT})_{15},(\mathrm{AT})_{15},\left(\mathrm{G}_{3} \mathrm{~T}\right)_{7}$, and $(\mathrm{T})_{30}$, and (b) four other polymers (PL-PEG-5k, PL-PEG-1.5k, PAA, and PAH). Adapted with permission from own work 55 . 


\subsubsection{Impact of redox-active molecules on the fluorescence changes}

After all samples were prepared, the emission spectra were collected before (black) and after (red) addition of the analytes of interest, as shown in Fig. 22. The time intervals between both spectra were in the range of 1-2 min to exclude aggregation or polymerization effects. All emission spectra were recorded in a Raman setup $\left(\lambda_{e x}=785 \mathrm{~nm}\right)$, with the detection range of 800-1050 nm. Therefore, the spectra exhibit both fluorescence and Raman features. The two Raman peaks correspond to: (1) the G-band $\left(1600 \mathrm{~cm}^{-1}, 890 \mathrm{~nm}\right)$ that is present in all $\mathrm{sp}^{2}$ hybridized carbon forms and arises from the planar, C-C stretching vibration, and (2) the G'-band $\left(2600 \mathrm{~cm}^{-1}, 960 \mathrm{~nm}\right)$ that is the second-order scattering process of the Dband and is present even in defect-free SWCNTs. A detailed discussion of typical SWCNTs Raman bands can be found in 2.1.4. The emission spectra also feature different SWCNTs chiralities, labeled with (n, m) indices (Fig. 22 a.)
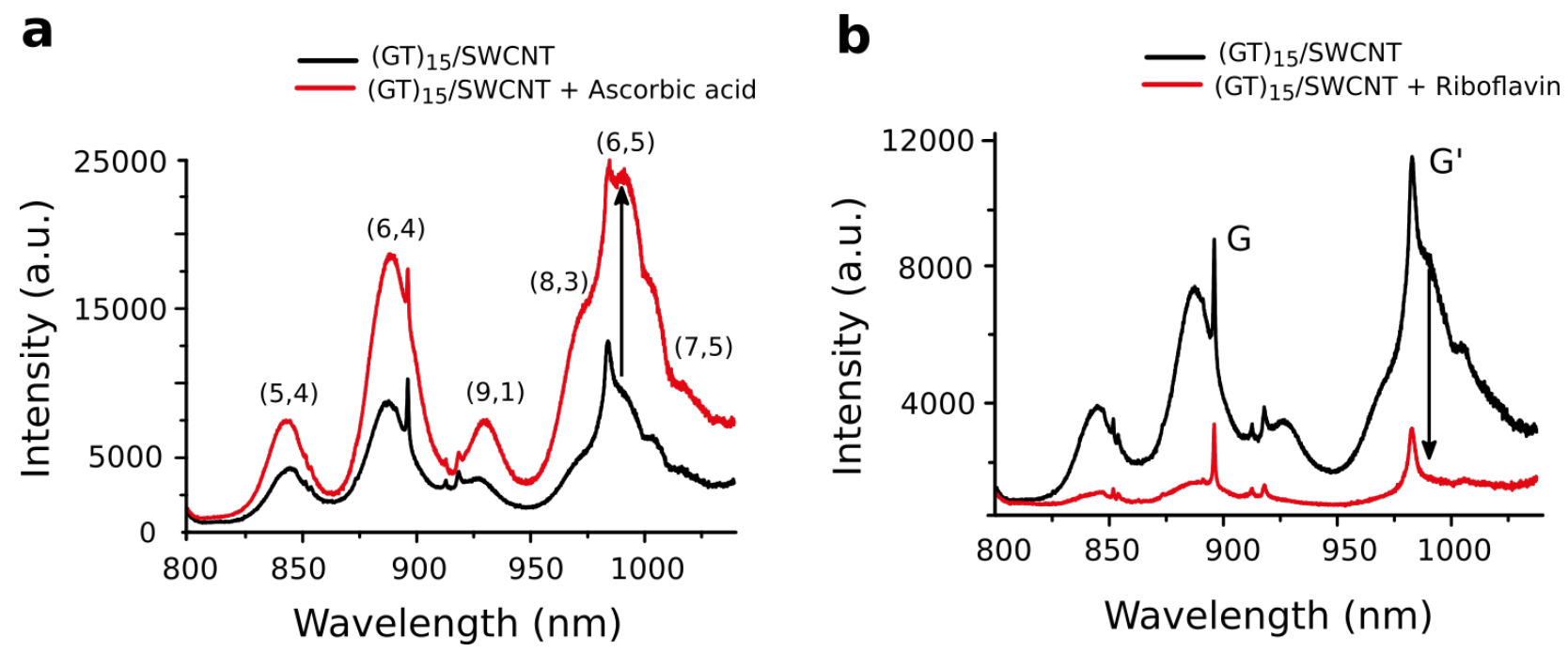

Figure 22: Fluorescence spectra of SWCNT/polymer complexes. (a) Fluorescence/Raman spectrum of $(\mathrm{GT})_{15} / \mathrm{SWCNT}$ before (black) and after (red) addition of ascorbic acid $(100 \mu \mathrm{M})$, different (n, m) indices label SWCNT species of different chirality, (b) The same $(\mathrm{GT})_{15} / \mathrm{SWCNT}$ complex but after addition of riboflavin $(100 \mu \mathrm{M})$. One can clearly see Raman features such as the G and G' peaks. Adapted with permission from own work [55].

As clearly demonstrated in the absorption spectra with the prominent peak around $990 \mathrm{~nm}$, the SWCNT sample was enriched in $(6,5)$ chirality (Fig. 21). Nevertheless, the fluorescence spectrum exhibits an unexpected increase in the intensity of smaller diameter SWCNTs, such as $(5,4),(6,4)$, and $(9,1)$. We attribute this intensity overestimation to two reasons. First, 
the excitation wavelength of the Raman setup $\left(\lambda_{e x}=785 \mathrm{~nm}\right)$ was off-resonance for the $(6,5)$ species. Second, instead of a more suitable nIR-sensitive detector, the setup was equipped with a Si-based detector. Si-based cameras slowly lose their sensitivity above 1000-1200 nm. Instead, permanent nIR setups usually employ indium-gallium-arsenide (InGaAs) detectors for the range of $900-1800 \mathrm{~nm}$ or mercury-cadmium-telluride ( $\mathrm{HgCdTe})$ for the wavelengths above $1400 \mathrm{~nm}$ [60]. The Si-based detector of the Raman setup had low quantum efficiency for the longer wavelengths and was more sensitive to signals below $1000 \mathrm{~nm}$. Therefore, our fluorescence spectra exhibit high intensity for such chiralities as $(5,4),(6,4),(9,1)$, and $(8,3)$. Due to the absorption spectrum we still know that our samples predominately contain $(6,5)$-SWCNTs. Hence, we will first focus on the analysis of this most abundant species.

Figure 21a demonstrates how the addition of the (reducing) ascorbic acid (with the end concentration of $100 \mu \mathrm{M})$ increases the fluorescence of $(6,5)$-SWCNTs up to $141 \%$. In comparison, in Figure 21b the fluorescence decreases down to $-81 \%$ after addition of (oxidizing) riboflavin. For the analysis, the fluorescence change was defined as the normalized intensity change at the (6,5)-SWCNT fluorescence maximum of $\lambda=990 \mathrm{~nm}$ :

$$
\frac{I-I_{0}}{I_{0}}=\frac{\Delta I}{I_{0}}
$$

with $I_{0}$ as the initial intensity of $(6,5)$-SWCNTs and $I$ as the intensity after addition of analytes. Normalized fluorescence changes are shown in the heat map in Figure 23. 


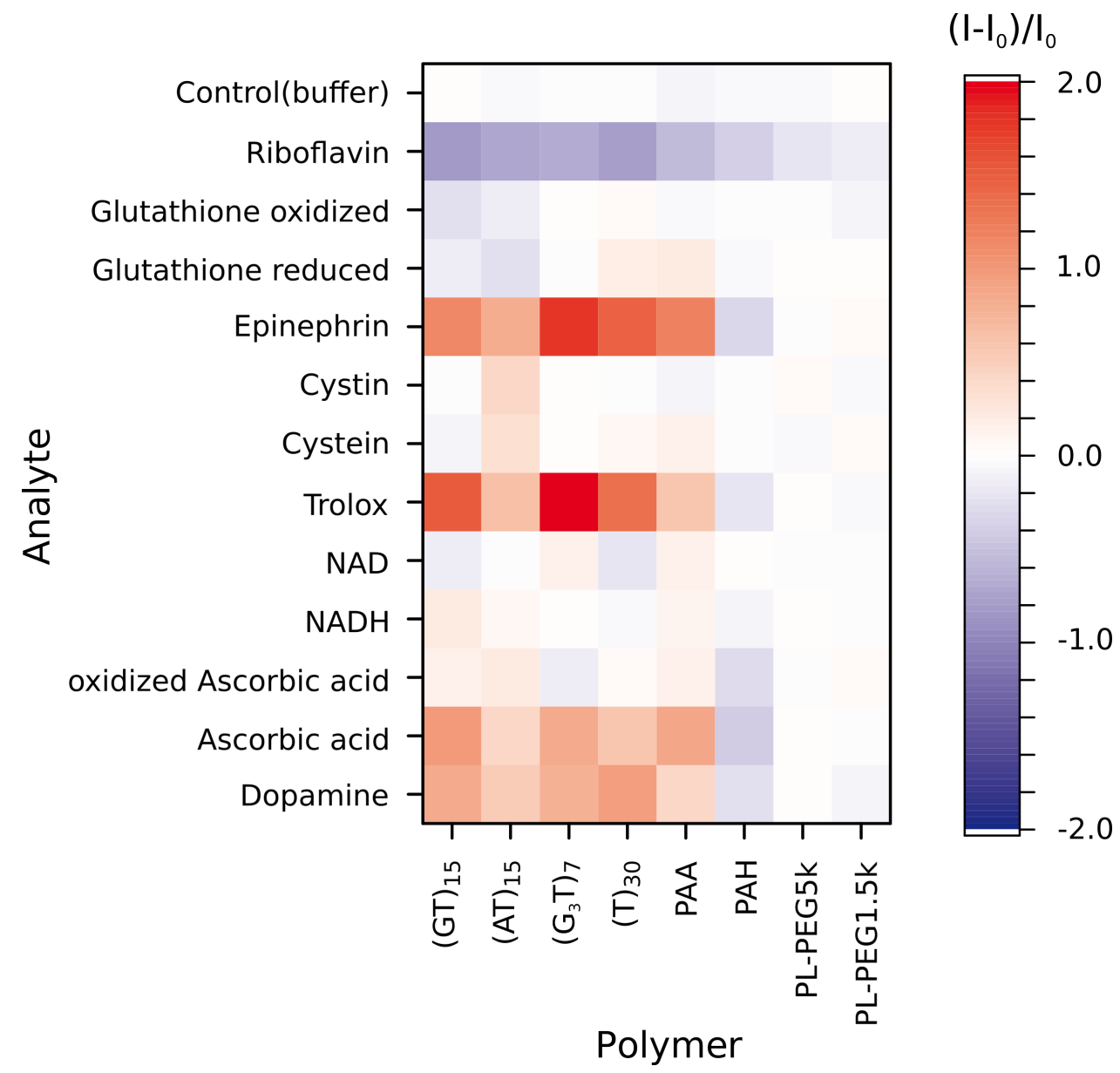

Figure 23: Heat map of fluorescence changes after addition of analyte to polymer/SWCNTs. Color-coded heat map of normalized fluorescence changes $\left(I-I_{0}\right) / I_{0}$ of polymer/SWCNTs upon addition of different analytes $(100 \mu \mathrm{M})$. Intensity change is color-coded $\left(\frac{\Delta I}{I_{0}}>0\right.$, red; $\frac{\Delta I}{I_{0}} \approx 0$, white; $\frac{\Delta I}{I_{0}}<0$, blue). Polymer/SWCNTs complexes are shown along the $\mathrm{x}$-axis and analyte molecules along the y-axis. Adapted with permission from own work [55].

If the results are analyzed from the polymer perspective, we can highlight several findings:

- All DNA-wrapped SWCNTs reacted in a similar manner regardless of their sequence. While their response pattern was alike, their absolute intensity varied. $\left(\mathrm{G}_{3} \mathrm{~T}\right)_{7}$ showed the highest fluorescence change, followed by $(\mathrm{GT})_{15}$ and $(\mathrm{T})_{30}$. $(\mathrm{AT})_{15}$ was the least responsive of all DNA sequences. On the one hand, guanine is known to act as an electron sink 235], which might explain the high responses. This explanation would support a sensing mechanism based on a redox reaction. On the other hand, the affinity of different DNA strands to SWCNT depends on the sequence. By pulling various DNA sequences from the SWCNTs surface, Iliafar et al. established following 
ranking for the binding strength of the four homopolymeres: $\operatorname{poly}(\mathrm{T})>\operatorname{poly}(\mathrm{A})>$ $\operatorname{poly}(\mathrm{G}) \geq \operatorname{poly}(\mathrm{C})$, with values ranging from 11.3 to $7.58 k_{B} T$ per nucleotide [138. Based on this information one might suggest that high fluorescence change is enabled by a medium binding strength between ssDNA and SWCNT surface. If the binding is too weak, SWCNT might not 'see' conformational changes of DNA. If it is too tight, DNA might have difficulties to change its conformation after the analyte binding. Another interesting finding is that all DNA/SWCNTs increased their fluorescence in the presence of most reducing molecules, but not all of them.

- Negatively charged polymer PAA showed a comparable response pattern as DNA. Especially the fluorescence increase by reducing molecules follows the same profile. But contrary to DNA it is difficult to imagine which functional groups in PAA could be reduced by these molecules.

- Interestingly, positively charged polymer PAH showed an inverted response pattern. That is especially interesting in the case of reducing molecules which caused a measurable fluorescence decrease.

- As expected, the inert PL-PEG/SWCNTs did not respond to any analyte. The only small decreases were observed in the case of riboflavin.

These results indicate that polymer chemistry is very important for the fluorescence change of SWCNT-based sensors. The strongest fluorescence changes were recorded for ascorbic acid, epinephrine, dopamine, and trolox $(141 \pm 39 \%, 114 \pm 16 \%, 84 \pm 8 \%$, and $151 \pm$ $22 \%$ for $(\mathrm{GT})_{15} / \mathrm{SWCNT}$ complexes). Other reducing agents, such as NADH or glutathione, showed only small changes $(21 \pm 5 \%, 14 \pm 3 \%)$. Compared to their oxidized equivalents, only ascorbic acid showed a consistent fluorescent increase that was stronger than that for oxidized ascorbic acid (141 $\pm 39 \%$ for ascorbic acid and $15 \pm 12 \%$ for the oxidized equivalent). For the other pairs, such as NAD/NADH, cysteine/cysteine, and glutathione/oxidized glutathione, we did not find a clear trend ( $14 \pm 2 \%$ vs $21 \pm 5 \%$ for NAD/NADH, $8 \pm 7 \%$ vs $2 \pm 6 \%$ for cysteine/cystin, and $14 \pm 3 \%$ vs $26 \pm 3 \%$ for glutathione/oxidized glutathione). Surprisingly, even oxidized ascorbic acid increased the fluorescence in certain cases. In contrast, riboflavin decreased the fluorescence of all tested polymer/SWCNT complexes (-16\% to -81\%). Even phospholipid PEG/SWCNT complexes which did not respond to any other reducing or oxidizing molecules were slightly quenched $(-16 \pm 2 \%)$. 

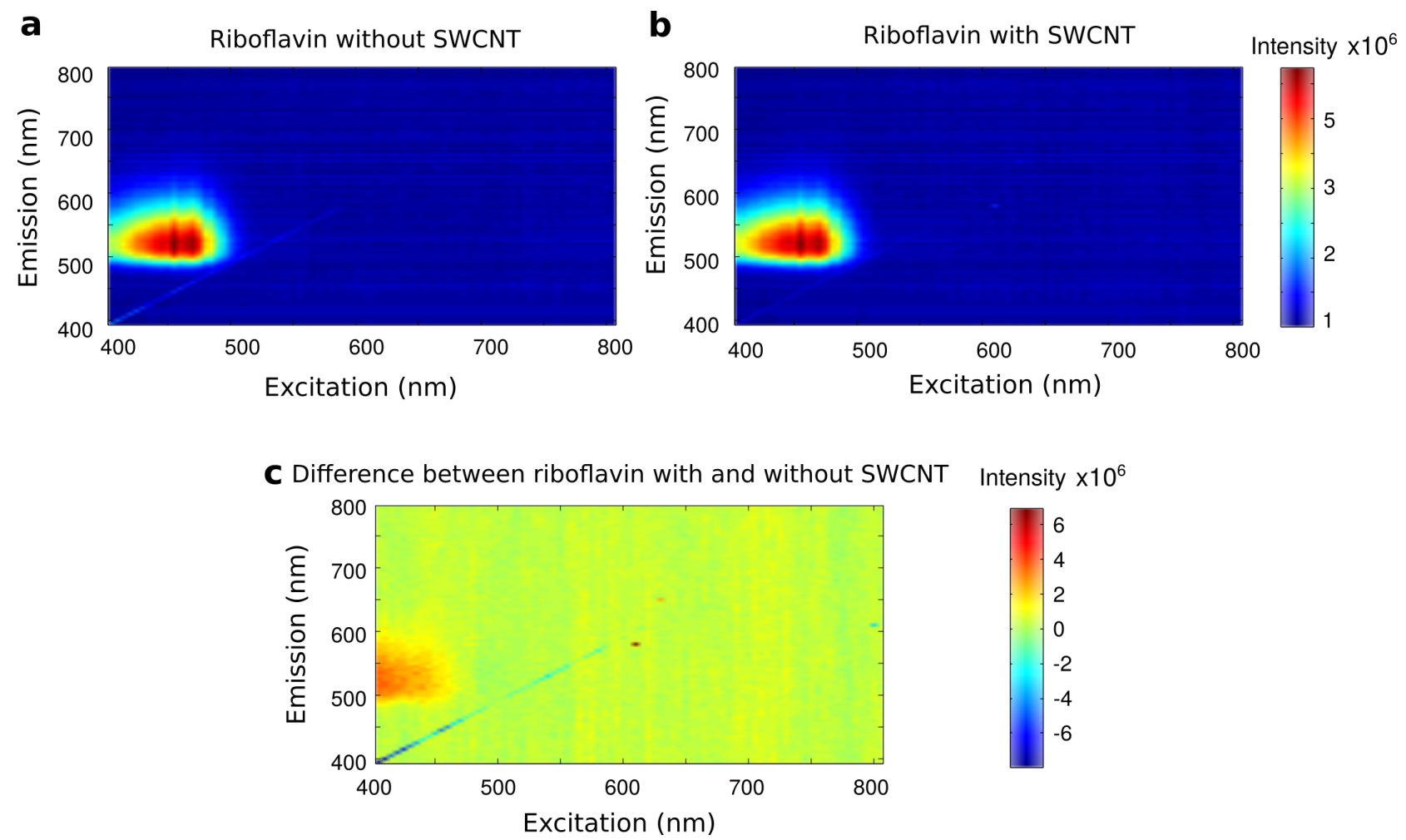

Figure 24: 2D excitation-emission spectrum of riboflavin (a) without and (b) with $(\mathrm{GT})_{15} / \mathrm{SWCNT}$, (c) The difference between spectra (a) and (b).

Fluorescence decrease for riboflavin is unique in comparison to other tested analytes. The molecule riboflavin is also a special case because it is the only molecule of our selection of analytes that displays fluorescence and absorbs light in the visible range (with $\lambda_{\text {ex,ribo }}$ $=520 \mathrm{~nm})$, almost at the same wavelength as $(6,5)$-SWCNTs $\left(\lambda_{e x, C N T}=560 \mathrm{~nm}\right)$. To further investigate the decrease in sensor fluorescence, we recorded a $2 \mathrm{D}$ excitation-emission spectrum of riboflavin with and without $(\mathrm{GT})_{15} / \mathrm{SWCNTs}$ (Figure 24a,b). The intrinsic fluorescence of riboflavin lies in the visible range between 500 and $600 \mathrm{~nm}$. This fluorescence is enhanced by the presence of SWCNTs (Figure 24c). Therefore, it is likely that riboflavin gets brighter due to the energy transfer from SWCNTs, while these simultaneously become dimmer (= decreasing fluorescence response). However, the absorption spectrum of riboflavin and the emission spectrum of SWCNTs do not overlap, which rules out FRET. 

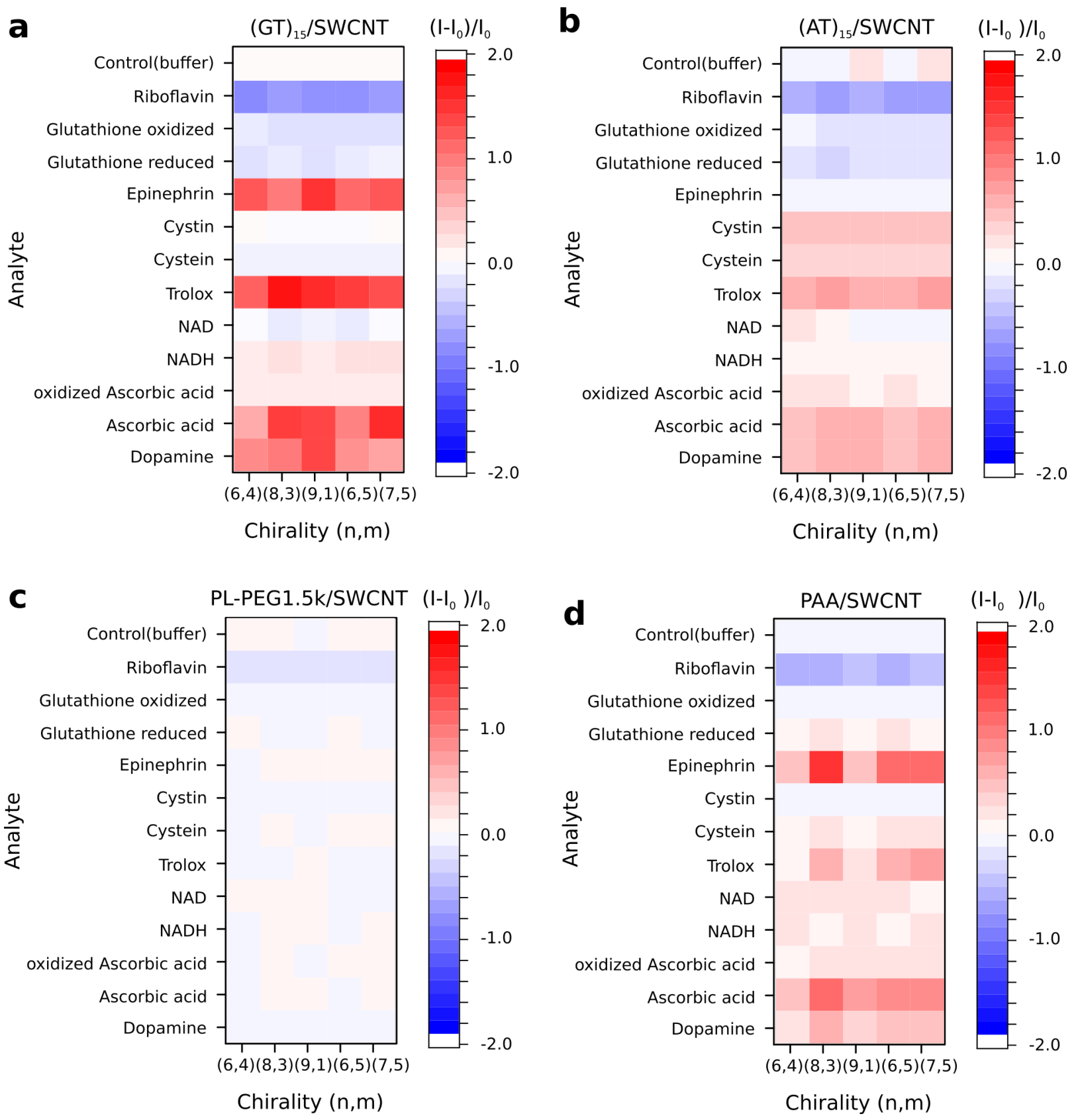

Figure 25: Fluorescence responses of different SWCNT chiralities. Heat maps with responses of different polymer/SWCNT complexes to redox-active molecules (red $=$ increase, blue $=$ decrease). The $\mathrm{x}$-axis displays different SWCNT chiralities, denoted with chiral index (n, m). The panels (a-d) correspond to different polymer wrappings: (a) $(\mathrm{GT})_{15}$-ssDNA, (b) (AT) ${ }_{15}$-ssDNA, (c) PL-PEG1.5k, and (d) PAA.

Redox potential of a SWCNT depends on its chirality [236]. With an increasing diameter of a SWCNT its oxidation potential decreases [237]. Therefore, we would expect a correlation between the fluorescence modulation and SWCNT chirality. SWCNT chiralities that are 
present in our sample are: $(6,4),(9,1),(8,3),(6,5)$, and $(7,5)$. Their diameters and the respective fluorescence responses after addition of dopamine $(100 \mu \mathrm{M})$ are shown in Table 3 .

Table 2: Calculated diameters of SWCNT chiralities and their respective normalized fluorescence responses $\Delta I / I_{0}$ after dopamine addition $(100 \mu \mathrm{M})$, analyzed for $(\mathrm{GT})_{15} / \mathrm{SWCNT}$.

\begin{tabular}{lll} 
Chirality & Diameter $(\mathbf{n m})$ & $\Delta I / I_{0}$ \\
\hline$(6,4)$ & 0.68 & 0.9 \\
$(9,1)$ & 0.74 & 1.4 \\
$(6,5)$ & 0.74 & 0.8 \\
$(8,3)$ & 0.77 & 1.1 \\
$(7,5)$ & 0.81 & 0.7
\end{tabular}

Normalized fluorescence changes for different SWCNT chiralities vary slightly around a similar value without a clear trend. There is also no impact of chirality on florescence response in other SWCNT/polymer complexes, as shown in Figure 25c-d. Changes of the free carrier concentration would have an influence on both SWCNT absorption and fluorescence spectra. We ruled out this possibility by collecting absorption spectra before and after the addition of an analyte. Typical absorption spectra after the addition of ascorbic acid or riboflavin are demonstrated in Figure 26 and show no change after the addition of these analytes. The unchanged absorption spectra also rule out that a change in SWCNT aggregation state could be responsible for the fluorescence response. In combination, both data sets indicate that the sensing mechanism is not based on a direct stoichiometric electron transfer between the analyte and the SWCNT.
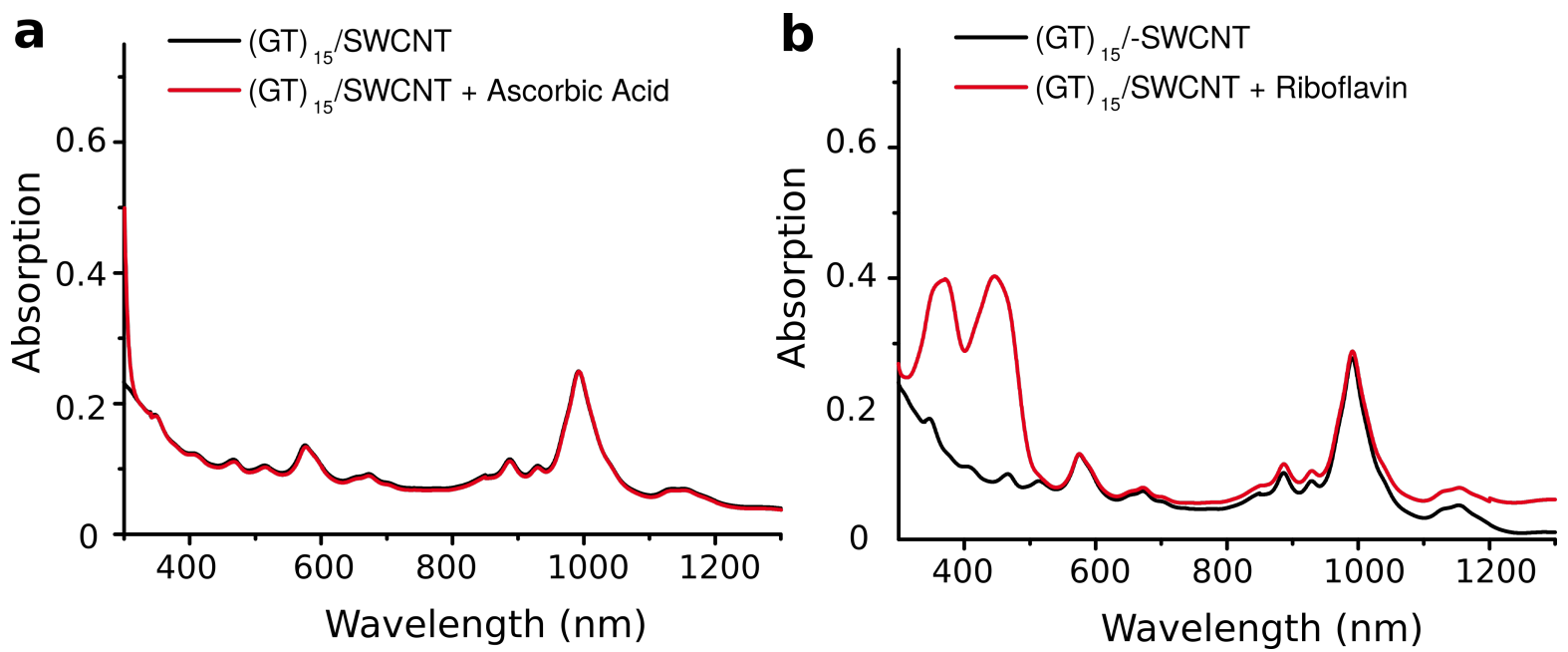

Figure 26: Typical absorption spectra after addition of an analyte to $(\text { GT })_{15} /$ SWCNT for (a) ascorbic acid, and (b) riboflavin. 


\subsubsection{Influence of reactive oxygen scavengers on fluorescence modulation}

At this point it is relevant to consider an important side effect of introducing reduction agents into the solution. Reactive oxygen species (ROS) are also known to quench ssDNA/SWCNT fluorescence 238]. Reducing agents can decrease ROS concentration in a solution. It is crucial to verify that the PL increase does not come from a dopamine-induced decrease of ROS concentration. One way to eliminate this possibility is to measure a Raman spectrum before and after the addition of an analyte. Any changes in D/G Raman peak ratio would indicate an oxidation of SWCNT or any change or defect in the electric structure of the sidewalls. Another possibility would be to perform experiments in the presence of ROS scavengers.

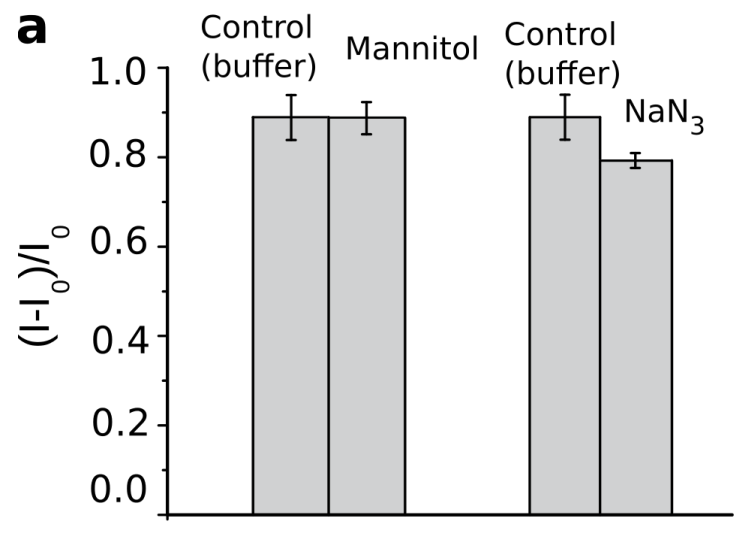

+ Ascorbic acid

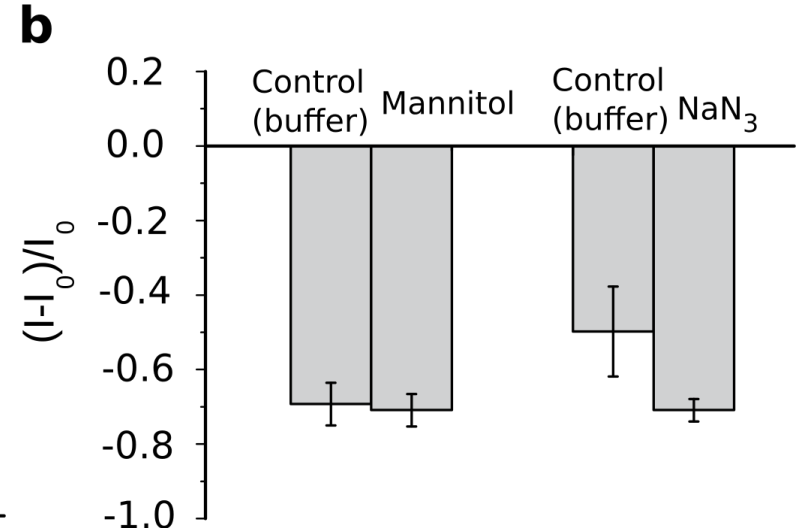

+ Riboflavin

Figure 27: Influence of reactive oxygen species. Addition of the singlet oxygen scavenger sodium azide $\left(\mathrm{NaN}_{3}\right)$ and the hydroxyl $(\mathrm{OH})$ radical scavenger mannitol to a $(\mathrm{GT})_{15} / \mathrm{SWCNT}$ solution and the subsequent addition of (a) ascorbic acid or (b) riboflavin $(100 \mu \mathrm{M})$ to the sensors. Normalized fluorescence change of $(6,5)$ peaks was analyzed. Error bars are standard deviations $(\mathrm{n}=3)$.

There are conflicting reports of both cases where SWCNTs produce or quench (consume) ROS [239], 240]. However, there is consensus about the fact that ROS (such as hydroxyl radicals, hydrogen peroxide or singlet oxygen) reduce fluorescence of SWCNTs [241], [242]. Therefore, it is possible that a reducing molecule, such as ascorbic acid, could decrease local ROS concentration. A lesser concentration of a quenching molecule would consequently increase SWCNT fluorescence. To test this hypothesis, we conducted experiments both in the presence of known ROS scavengers and without them. We used hydroxyl radical scavenger mannitol $(100 \mu \mathrm{M})$ and singlet oxygen scavenger sodium azide $\left(\mathrm{NaN}_{3}, 100 \mu \mathrm{M}\right)$. First, we monitored possible changes after the addition of ROS to a $(\mathrm{GT})_{15} / \mathrm{SWCNT}$ solution. Then we recorded if there is any difference between the response of standard $(\mathrm{GT})_{15} / \mathrm{SWCNT}$ 
sensors to analyte vs the response of ROS $+(\mathrm{GT})_{15} / \mathrm{SWCNT}$ to the same analyte. As shown in Figure 27, the presence of scavengers had no $(<2 \%$ for ascorbic acid and mannitol $)$ or only a small impact ( $10 \%$ for ascorbic acid and $\mathrm{NaN}_{3}$ ) on fluorescence changes. If ROSscavenging were involved in the sensing mechanism, one would expect either a bigger change or that the ROS $+(\mathrm{GT})_{15} / \mathrm{SWCNT}$ sensors would become insensitive to analytes. Since there was no or very little variation between samples with oxygen scavengers and without (control), we can exclude that fluorescence response after addition of redox-active molecules is caused by scavenging of ROS.

\subsubsection{Impact of polymer surface coverage on polymer/SWCNT response}

Aside from the mechanism of the direct electron transfer between analyte and SWCNT or ROS scavenging, another proposed mechanism is based on a direct adsorption of an analyte onto the surface of SWCNTs. To verify this hypothesis, we tested if there is a correlation between the free surface area of a SWCNT and fluorescence changes. To estimate the surface coverage of polymer/SWCNTs we used the solvatochromic shift method. This theory is based on the finding that energy separation (band gap) is a function of SWCNT's diameter (chirality). Following this consideration, the positions of local energy maxima ('local' values of $\mathrm{S}_{i i}$ within the same SWCNT species) depend on the surrounding environment. Therefore, the relationship between the band gap and the diameter (chirality) of SWCNTs is mediumdependent. The general idea is that the solvatochromic shift $\Delta E_{i i}$ reflects how much of SWCNT surface is covered by polymer and how much by solvent 243. In the following, we will reconstruct the pathway of the surface coverage calculation step by step.

Fluorescent emission peaks for each $(n, m)$ chirality reflect the radiative bandgap of semiconducting SWCNTs. Under influence of the local dielectric environment their transition energy changes, thus producing a measurable change in optical transitions. To determine the exact change in the energies, we first have to define the reference. The reference $E^{a i r}$ can be observed for SWCNTs in the air or in any other medium with the dielectricity constant $\varepsilon \approx 1$. The solvatochromic shift $\Delta E_{i i}$ reflects the difference between the optical transition energy in the polymer environment and in the air:

$$
\Delta E_{i i}=\left(E_{i i}^{a i r}-E_{i i}^{\text {polymer }}\right)
$$

$E_{i i}^{\text {polymer }}$ was determined from the absorption spectra of polymer/SWCNTs (Fig. 21). $E_{i i}^{\text {air }}$ was calculated using formula from [243]:

$$
E_{i i}^{a i r}=\frac{h c}{A_{1}+A_{2} d}+A_{3} \frac{\cos 3 \theta}{d^{2}}
$$


where $h$ is Plank's constant, $c$ is the speed of light, $d$ is the SWCNT diameter and $\theta$ is the chiral angle of the tube. $A_{1}, A_{2}$ and $A_{3}$ are parameters scaled to fit the data for $\varepsilon \approx 1$. Here, we only consider $\mathrm{S}_{11}$, for other optical transitions there are different parameters. For $\mathrm{S}_{11}$ the parameters are: $A_{1}=61.1 \mathrm{~nm}, A_{2}=1113.6 \mathrm{~nm}$, and the value for $A_{3}$ depends on $\bmod ((n-m), 3)=\mathrm{j}$.

$$
\mathrm{A}_{3}= \begin{cases}-0.077 \mathrm{eVnm}^{3}, & \text { for } j=1 \\ -0.032 \mathrm{eVnm}^{3}, & \text { for } j=2\end{cases}
$$

Both $d$ and $\theta$ can be calculated for each chirality species with formula from Table 1:

$$
\begin{aligned}
& d=\frac{a}{\pi} \sqrt{n^{2}+n \cdot m+m^{2}} \\
& \theta=\arctan \frac{\sqrt{3} m}{\sqrt{(2 n+m)}}
\end{aligned}
$$

Based on these calculations, we can determine optical transitions $\mathrm{S}_{11}$ of SWCNTs in the air:

Table 3: Optical transitions $\mathrm{S}_{11}$ of SWCNTs in the air.

\begin{tabular}{ll} 
Chirality & $E_{i i}^{a i r} \mathbf{e V}$ \\
\hline$(6,4)$ & 1.48 \\
$(9,1)$ & 1.43 \\
$(6,5)$ & 1.48 \\
$(8,3)$ & 1.37 \\
$(7,5)$ & 1.25
\end{tabular}

For further calculation we need to discuss exciton polarizability of SCWNTs in various media. SWCNTs have no intrinsic dipole moment, but a dipolar solvent around a nanotube can induce one. The wavelength shift observed in fluorescence peaks is proportional to the difference in polarizability between pristine SWCNTs in the air and SWCNTs in solution. The change in polarizability $\Delta \alpha_{i i}$ correlates with the difference between the ground state and the excited state for $E_{i i}$ transitions. Assuming that the solvatrochromic shift is proportional to Onsager polarity function $f(x)=2(x-1) /(2 x+1)$, the solvatrochromic shift $\Delta E_{i i}$ can be described as [244]:

$$
\Delta E_{i i}=-L \frac{\Delta \alpha_{i i}}{r^{3}}\left[\frac{2(\varepsilon-1)}{2 \varepsilon+1}-\frac{2\left(\eta^{2}-1\right)}{2 \eta^{2}+1}\right]
$$


With $L$ as the fluctuation factor, $r$ as the SWCNT radius, $\varepsilon$ as the solvent dielectric constant and $\eta$ as the solvent refractory index. This semi-empirical model links the optical transition energies $\Delta E_{i i}$ with the exciton polarizability of SWCNTs in various media and the chirality of SWCNTs.

There are several experimental and theoretical works concerning the exact expression for polarizability of excitons on nanotubes. The reaction field of the solvent depends on the size of the nanotube. Therefore, the general functional form for polarization of nanomaterials (such as nanotubes and quantum dots) is presented as $\Delta \alpha_{i i}=k r^{a}\left(\Delta E_{i i}\right)^{b}$, with $a$ and $b$ depending on the exact geometry of the nanomaterial in question. For SWCNTs those parameters are assumed to be $\mathrm{a}=-1$ and $\mathrm{b}=-2$ [245]. This leads to the final expression of $\Delta \alpha_{i i}=k r^{-1}\left(\Delta E_{i i}\right)^{-2}$. By inserting this in equation (22) we get the final formula:

$$
\left(E_{11}\right)^{2} \Delta E_{11}=-L k\left[\frac{2(\varepsilon-1)}{2 \varepsilon+1}-\frac{2\left(\eta^{2}-1\right)}{2 \eta^{2}+1}\right]\left(\frac{1}{r^{4}}\right)
$$

which can be simplified to:

$$
\left(E_{11}\right)^{2} \Delta E_{11}=\frac{c}{r^{4}}
$$

with $c$ as the parameter which defines the solvent's influence. This characteristic constant can be obtained by plotting $\left(E_{11}\right)^{2} \Delta E_{11}$ against $1 / r^{4}$. This results is a linear graph with positive slope $c$. Energy shift shows monotonic increasing trend with decreasing diameter. After determining $c$ there are still two unknown factors: $L$ and $k$. One can calculate these by comparing the slope $c$ of the new polymer to that of a reference substance. For historical reasons, the reference substance is n-methyl-2-pyrrolydone (NMP). Parameters for that system are: $\mathrm{c}_{N M P}=0.060 \mathrm{eV}^{3} \mathrm{~nm}^{4}, \varepsilon_{N M P}=32.2$ and $\eta_{N M P}=1.47$.

$$
\frac{c}{c_{N M P}}=\frac{\frac{2\left(\varepsilon_{e f f}-1\right)}{2 \varepsilon_{e f f}+1}-\frac{2\left(\eta_{e f f}^{2}-1\right)}{2 \eta_{e f f}^{2}+1}}{\frac{2\left(\varepsilon_{N M P}-1\right)}{2 \varepsilon_{N M P}+1}-\frac{2\left(\eta_{N M P}^{2}-1\right)}{2 \eta_{N M P}^{2}+1}}
$$

Using parameters for a NMP-SWCNT system, the divisor was calculated to be 0.2589. After transposing, formula 25 is simplified to:

$$
\frac{0.2589 c}{0.06 \mathrm{eV}^{3} \mathrm{~nm}^{4}}=\frac{2\left(\varepsilon_{e f f}-1\right)}{2 \varepsilon_{e f f}+1}-\frac{2\left(\eta_{e f f}^{2}-1\right)}{2 \eta_{e f f}^{2}+1}
$$

Generally, the refractory index of the wrapping polymer $\eta_{\text {eff }}$ is assumed to be the same as 
the refractory index of water $\left(\eta_{\text {water }} \approx 1.33\right)$. After inserting this in 26 we get:

$$
\frac{0.2589 c}{0.06 \mathrm{eV}^{3} \mathrm{~nm}^{4}}=\frac{2\left(\varepsilon_{e f f}-1\right)}{2 \varepsilon_{e f f}+1}-0.3412
$$

$c$ was obtained from plotting $\left(E_{11}\right)^{2} \Delta E_{11}$ against $1 / r^{4}$. By inserting $c$ into the equation (27), we can calculate the effective dielectric constant $\varepsilon_{\text {eff }}$ experienced by the SWCNT. $\varepsilon_{\text {eff }}$ can also be described by an interpolation of the dielectric constants of water $\left(\varepsilon_{\text {water }}\right)$ and of the wrapping polymer $\left(\varepsilon_{p}\right)$ :

$$
\varepsilon_{e f f}=\alpha \varepsilon_{p}+(1-\alpha) \varepsilon_{\text {water }}
$$

Hence, we can calculate the relative surface coverage $\alpha$. Known factors are: $\varepsilon_{\text {eff }}$ from plotting of $\left(E_{11}\right)^{2} \Delta E_{11}$ against $1 / r^{4}$ and calculating $c$; $\varepsilon_{p}$ as the dielectric constant of the wrapping polymer ( $\varepsilon_{p}=4$ for DNA, $\varepsilon_{p}=3$ for PAH, $\varepsilon_{p}=6 \mathrm{PAA}, \varepsilon_{p}=2.1$ for phospholipid); and $\varepsilon_{\text {water }}$ as the dielectric constant of water $\left(\varepsilon_{\text {water }}=88\right)$ [246], 247], 248], 249]. Simple rearrangement leads to:

$$
\alpha=\frac{\varepsilon_{\text {eff }}-\varepsilon_{\text {water }}}{\varepsilon_{p}-\varepsilon_{\text {water }}}
$$

The results are presented in Figure 28 and in more detail in Table 4.

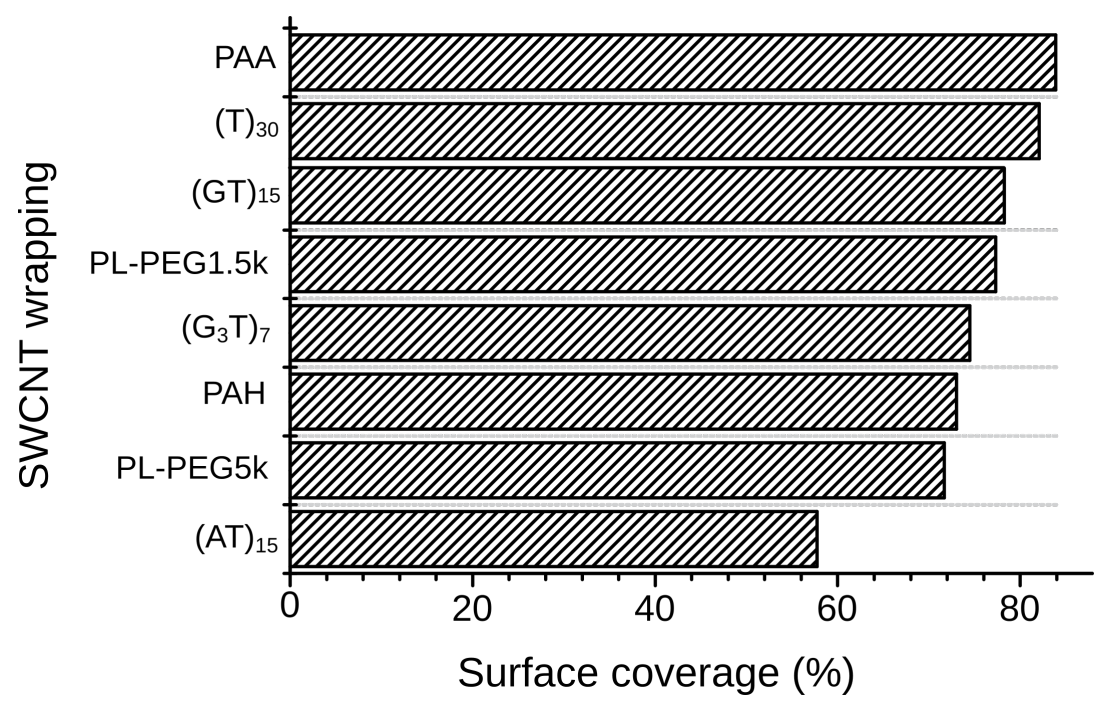

Figure 28: Surface coverage of different polymer/SWCNT complexes calculated by analyzing solvatochromic shifts.

When we focus on DNA with different sequences, the surface coverage $\alpha$ spans from $58 \%$ for $(\mathrm{AT})_{15}$ to $82 \%$ for $(\mathrm{T})_{30}$. Yet the corresponding fluorescence changes are very similar: e.g. $53 \pm 9 \%$ for addition of ascorbic acid to (AT) ${ }_{15} /$ SWCNT and $58 \pm 15 \%$ for addition of ascorbic acid to $(\mathrm{T})_{30} / \mathrm{SWCNT}$. The PL-PEG/SWCNT complexes exhibit comparable 
surface coverages (77\% for PL-PEG-1.5k and $72 \%$ for PL-PEG-5k) but showed nearly no response to ascorbic acid ( $2 \pm 0.4 \%$ for PL-PEG-1.5k and $1 \pm 0.1 \%$ for PL-PEG-5k).

Table 4: Solvatochromic shifts for all polymer/SWCNT combinations, with the dielectric constant $\varepsilon_{e f f}$ and the relative surface coverage $\alpha$ for each polymer wrapping.

\begin{tabular}{cccc} 
Polymer & Slope $c\left[\mathrm{eV}^{3} \mathrm{~nm}^{4}\right]$ & $\varepsilon_{\text {eff }}$ & $\alpha[\%]$ \\
\hline PAA & 0.067 & 18.39 & 84 \\
$\mathrm{~T}_{30}$ & 0.067 & 19.07 & 82 \\
$(\mathrm{GT})_{15}$ & 0.069 & 22.28 & 78 \\
$\mathrm{PEG}-1.5 \mathrm{k}$ & 0.068 & 21.61 & 77 \\
$\left(\mathrm{G}_{3} \mathrm{~T}\right)_{7}$ & 0.070 & 25.48 & 74 \\
$\mathrm{PAH}$ & 0.070 & 27.80 & 73 \\
$\mathrm{PEG}-5 \mathrm{k}$ & 0.070 & 26.44 & 72 \\
$(\mathrm{AT})_{15}$ & 0.072 & 39.54 & 58
\end{tabular}

Plotting normalized fluorescence change $\Delta I / I_{0}$ versus surface coverage shows no apparent correlation. Figure 29 demonstrates the data for ascorbic acid (a) and riboflavin (b). For example, in $\mathrm{PAH} / \mathrm{SWCNT}$ the surface coverage of $\mathrm{PAH}$ amounts to $73 \%$ and the complex shows a negative response of $-43 \% \pm 8 \%$ to ascorbic acid. At the same time, $\left(\mathrm{G}_{3} \mathrm{~T}\right)_{7} / \mathrm{SWCNT}$ complex has the same surface coverage of $74 \%$, but shows a strong positive response of 84 $\pm 6.8 \%$ to the same analyte. Instead of a correlation, the data points demonstrate random distribution in regard to surface coverage. These results suggest that the free surface available on the SWCNT surface is not a determining parameter for the sensing mechanism.
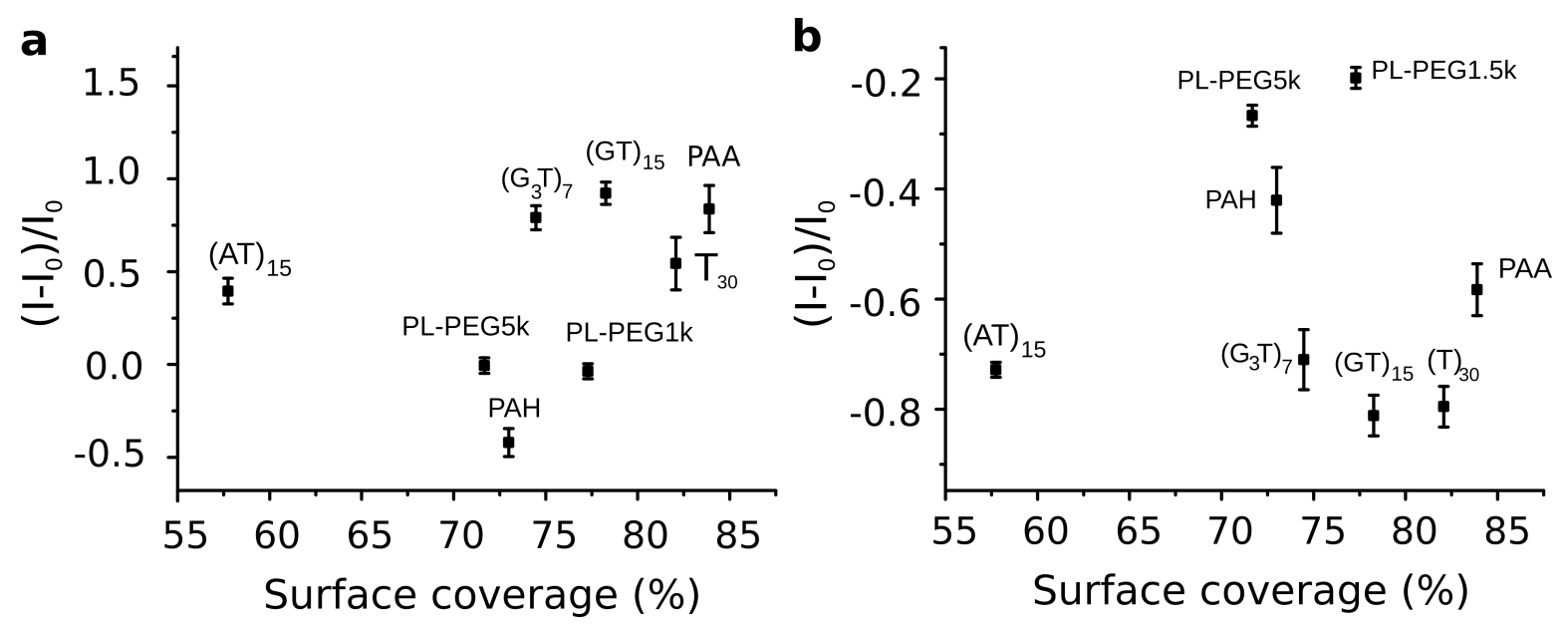

Figure 29: Fluorescence changes versus surface coverage. For (a) ascorbic acid (100 $\mu \mathrm{M})$ and (b) riboflavin $(100 \mu \mathrm{M})$. Error bars are standard deviations $(\mathrm{n}=3)$. 


\subsubsection{Influence of redox potential on SWCNT-sensor responses}

The chemical structures of three analytes which caused the biggest increases in SWCNT fluorescence are very different (ascorbic acid, trolox, and epinephrine). At the same time, their redox potentials are similar [250], 251], 252]. To investigate possible correlation between the responses of different polymer/SWCNTs and the redox potentials of analytes, we plotted both values as shown in Figure 30 a.
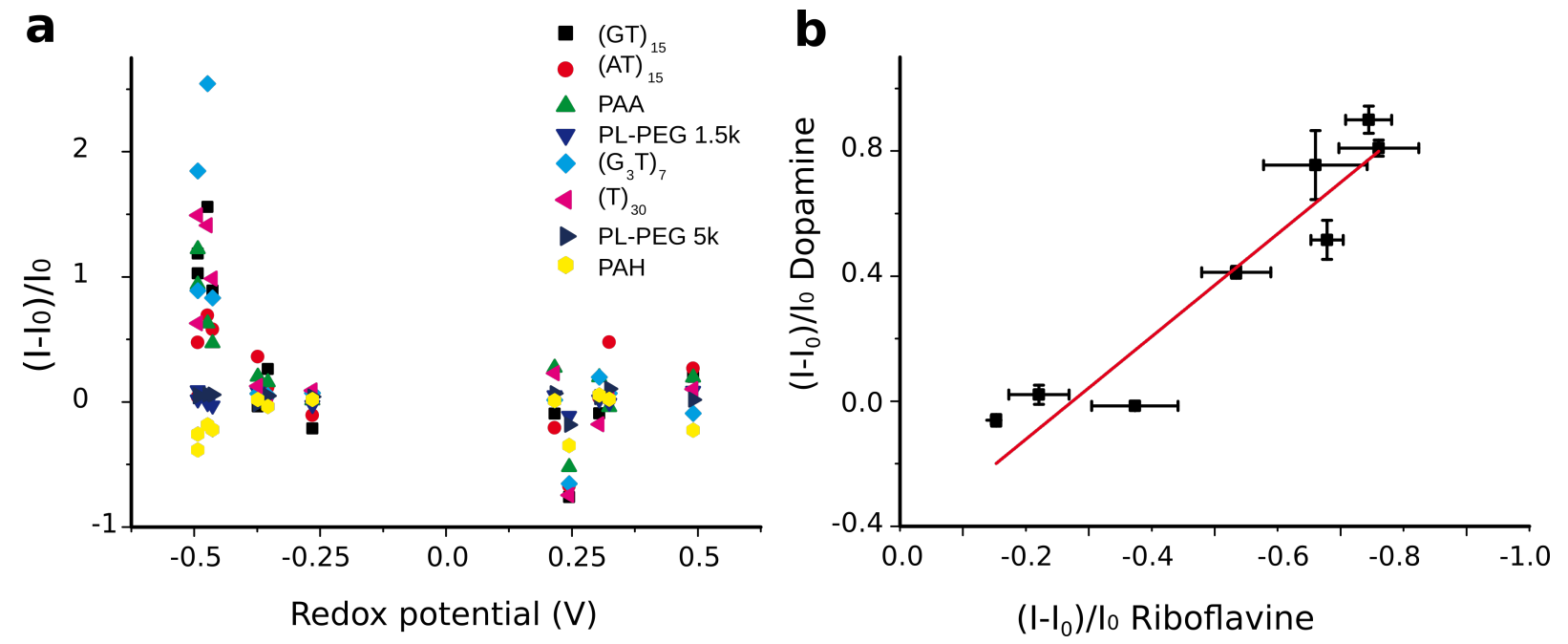

Figure 30: Correlation between redox potential of analytes and fluorescence response of polymer/SWCNTs. (a) Fluorescence changes of different polymer/SWCNT complexes as a function of redox potential. (b) Response to dopamine versus response to riboflavin for the eight different polymer/SWCNT complexes (red line is a linear fit, slope $\left.=1.6, R^{2}>0.8\right)$. Error bars are standard deviations $(\mathrm{n}=3)$.

The data show that molecules with a negative redox potential $<-0.4 \mathrm{~V}$ (e.g. dopamine, ascorbic acid, and trolox) increased the fluorescence of SWCNT-based sensors. Still, negative redox potential alone was not sufficient. There was a wide spread of fluorescence changes from $-50 \%$ to $250 \%$ depending on the polymer. Hence, we can conclude that reducing molecules are more likely to increase the fluorescence of SWCNTs than molecules with redox potential of $>-0.4 \mathrm{~V}$. Nevertheless, the exact magnitude of the sensor response depends on both the polymer and the structure of the molecule. For positive redox potentials, we did not find a clear tendency. Riboflavin decreased fluorescence of all samples, but as discussed before, this particular case was rather attributed to the light absorption of riboflavin in the same window as SWCNTs and an energy transfer. Interestingly, all polymer/SWCNTs that increased fluorescence in the presence of reducing molecules also decreased their fluorescence in the presence of oxidizing molecules. The linear relationship in Figure 30b suggests that 
both brightening and quenching effects might be related. Based on this, we introduced an empirical equation which predicts the fluorescence response of SWCNT-based sensors:

$$
\frac{\Delta I}{I_{0}}(c)=\delta(c) \beta
$$

The equation shows a dependence of normalized fluorescence change $\Delta I / I_{0}$ on the analyte concentration $c$. The dependence is described by a polymer and analyte dependent calibration curve $\delta(c)$ and an additional redox factor $\beta$. For negatively charged polymers, the redox factor $\beta$ can be either 1 (for reducing molecules) or -1 (for oxidizing molecules). For positively charged polymers $\beta$ could be adjusted accordingly. This empirical model includes aspects of a redox-based sensing mechanism and explains outliers (such as NADH, dopamine) that were not accounted for in a previously published 2D adsorption model of SWCNT-based sensors 253].

\subsubsection{Conclusion}

The underlying mechanism of the exact interaction between analyte and polymer/SWCNT is not fully understood [54, [101], 254]. In this chapter we performed complementary experiments to investigate four possible sensing mechanisms: (1) a pure adsorption-based mechanism, (2) a sensing mechanism with a major role of a redox-reaction oxidizing either the SWCNT (3) or the polymer, and (4) fluorescence change due to the conformational change of the polymer upon analyte binding.

Our data did not support a pure adsorption-based mechanism (1). We calculated the free area on the surface of SWCNTs for different polymer wrappings based on the solvatochromic shifts $\Delta E_{i i}$. This method is based on the idea that the optical properties of SWCNTs are medium depended. Our results demonstrated no correlation of polymer coverage and the observed fluorescence changes.

Second mechanism involves redox-reaction with the SWCNT (2) or the polymer (3). We were able to eliminate the involvement of reactive oxygen species (ROS) in the observed fluorescence change by adding them to our experiments. There was no significant change between 'normal' fluorescence responses and fluorescence responses for sensor + ROS mixtures. Electron transfer to and from the SWCNT itself was also excluded. Populating the conduction band or depopulating the valence band is known to quench both absorption and fluorescence emission 237], 255]. Our results showed no changes in the absorption spectra before and after the addition of an analyte. Furthermore, the response pattern was the same for all chiralities, which also indicates that the oxidation potential of SWCNT is not relevant for the sensing mechanism. Moreover, since the brightening of polymer/SWCNTs 
is reversible, it is not likely that these molecules introduce permanent covalent defects in the carbon lattice. Therefore, the fluorescence increase is not caused by the removal of dark excitons via covalent chemistry 104. Meanwhile, a mechanism that would non-covalently perturb the $\mathrm{sp}^{2}$ network is in agreement with the observed patterns. Hence, we can exclude a stoichiometric redox reaction, however a catalytic involvement of redox active species could still influence exciton dynamics.

Results for investigation of the direct electron transfer to or from the polymer (3) were mixed. For various DNA sequences this hypothesis would be sustainable. Guanine acts as an electron sink 235 and guanine-rich DNA wrapping showed the highest fluorescence change in our experiments. However, for this theory to be satisfactory demonstrated, it should explain other sensor responses by showing how the polymer is either accepting or donating the electrons. However, for PAA it is difficult to imagine which functional groups could be reduced. Moreover, since the fluorescence change is reversible, a possible backward reaction makes a purely redox mechanism improbable [54]. It is therefore possible that the redox mechanism is either not involved in the general sensing or only contributes to the overall florescence change in case of DNA-sequences and similar polymers.

The role of redox potential is ambiguous in our data. The highest responses were indeed observed for molecules with the lowest reduction potential $(<-0.4 \mathrm{~V})$. However, the inversion of the argument does not work, as not all molecules with that redox potential induce the same fluorescence response. Moreover, the specific fluorescence changes range from $+250 \%$ to $-50 \%$ depending on the exact wrapping polymer, which again emphasizes the import role of the organic phase. Therefore, our results support the idea that the redox potential is involved in the sensing mechanism, but the redox potential alone cannot explain the response patterns. Interestingly, the responses to reducing molecules also seem to correlate with the charge of the polymer. For negatively charged polymers we observed a fluorescence increase, while for positively charged polymer the same analytes caused a fluorescence decrease.

Finally, the last possible mechanism is a conformational change of the polymer that affects exciton decay routes. It was shown that small changes in molecule distribution around the SWCNT could change the exciton decay routes, and consequently the SWCNT fluorescence [256]. Experimentally, it is difficult to distinguish if a perturbation of the polymer around SWCNT is caused by a redox reaction or by any other interaction between the polymer and the analytes. The finding that PAA (with no apparent groups that could be reduced) is also sensitive to redox molecules supports this theory. Furthermore, the sensor response for PAA showed the same pattern as for DNA-wrappings, however with a lower magnitude. It is possible that in the case of PAA the interactions between functional groups and analyte are responsible for the conformational change of the polymer, while for DNA the conformational change is caused by both polymer-analyte interaction and a catalytic redox reaction. 


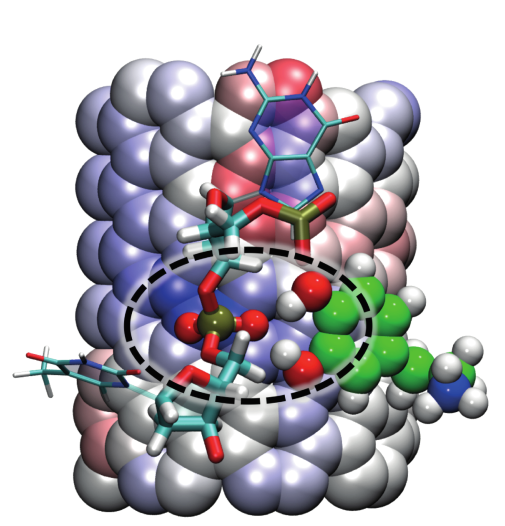

top

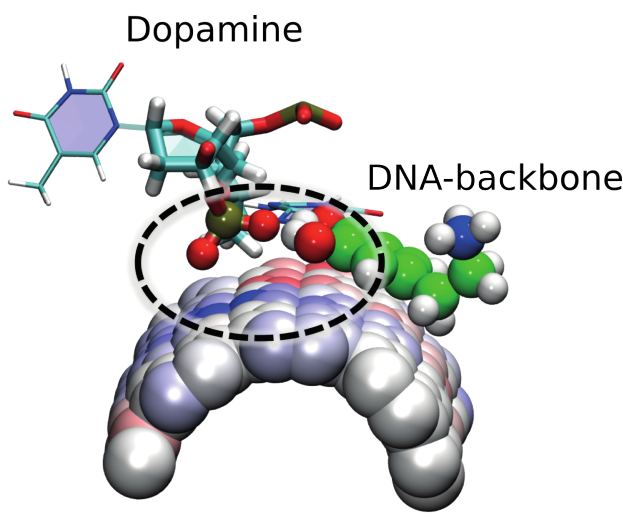

side<smiles>NCCc1ccc(O)c(O)c1</smiles>

Figure 31: Proposed sensing mechanism based on conformational change of the polymer around SWCNT. Dopamine pulls phosphate groups from the DNA-backbone towards the SWCNT surface. Phosphate groups then take the place of quenching sites which are thus removed. This shift increases SWCNT fluorescence (MD simulations). Adapted from [56].

Furthermore, MD simulations conducted by Lela Vukovic and Klaus Schulten support the hypothesis that interaction of the analyte with the wrapping polymer can change the local potential experienced by excitons and consequently change the fluorescence quantum yield of SWCNTs [56]. The proposed mechanism suggests that dopamine interacts via two hydroxy groups of the chatecholamine unit with the phosphate groups of the DNA backbone, as shown in Figure 31. The phosphate groups $\left(\mathrm{PO}_{4}^{-}\right)$are pulled closer to the surface of SWCNT and move the water molecules aside. The change in the local exciton potentials induced by dopamine binding lies in the range of several kcal $/ \mathrm{mol}$.

In summary, we demonstrated that the organic phase of SWCNT-based sensors plays a crucial role, especially in case of redox-active compounds. This work has both practical and theoretical implications. First, we created a library of several polymers and analytes to choose from for various applications. Second, we investigated several possible sensing mechanisms and our results support the explanation that observed response patterns are caused by conformational changes of the polymer around SWCNTs. 


\subsection{Development of sensors with tunable recognition units}

(Data presented in this chapter have been submitted to ACS Applied Materials $\mathcal{6}$

$$
\text { Interfaces.) }
$$

Following up on the previous section, we can summarize the advantages of SWCNT-based sensors [54], [56], [58], [59], [62]:

- high photostability (which is essential for experiments over extended periods of time and ensures reproducibility)

- tissue transparency for biological applications (deeper signal penetration and less tissue damage in comparison to fluorophores emitting in the visual range)

- large Stokes-shifts

- binding kinetics in the biologically relevant time regime (ms-range) to trail temporal concentration profiles of highly mobile neurotransmitter molecules after the release

- small dimensions, enough to be implemented into a sensor array and provide spatial information about the release sites $(<500 \mathrm{~nm})$

- reversibility of intensity change allows to trace diffusion profiles over time

- analyte sensitivity down to the single-molecule level (100 pM) for individual nanosensors and in $\mathrm{nM}$ range for sensor ensembles or in solutions

as well as their disadvantages [57], [60], [149]:

- low selectivity for chemically similar compounds (e.g. dopamine vs. epinephrine)

- low quantum yield and the need of nIR-sensitive equipment

- possible toxicity depending on the exact functionalization and SWCNT characteristics

As one can see, the advantages of SWCNT-based sensors are very suitable for sensor application and neurotransmitter detection (e.g. time scales and dimensions). Several of the disadvantages can be improved. Low quantum yield can be increased via defects or superior purification methods. Toxicity can be decreased by using shorter SWCNTs and a suitable functionalization. But the sensor selectivity still remains a hindrance on the way to biological applications. Some sensors, such as $(\mathrm{GT})_{10} / \mathrm{SWCNT}$, can distinguish between different catecholamines (such as dopamine and norepinephrine) at low concentrations of interfering molecules [57]. But this selectivity is not enough to detect dopamine at a high environmental concentration of ascorbic acid. Therefore, it is essential to develop sensors that combine the sensitivity of known ssDNA/SWCNTs sensors with sufficient selectivity. 
Proteins and peptides are well-known for their specific molecular interactions in nature, but have rarely been used for SWCNT functionalization due to colloidal stability issues [166]. One method to combine ssDNA and peptides in the organic phase of SWCNT-based sensors would be to conjugate ssDNA via linker to a peptide recognition unit and adsorb the whole macromolecule on a SWCNT, as shown in Fig. 32, This strategy not only allows to create highly selective and sensitive sensors but can also be tuned via small changes of one of its components: DNA (sequence, length, the strength of DNA binding to the SWCNT surface, etc.), linker (rigidity, length, curvature, etc.) or by varying the flanking peptides around the recognition unit.

\subsubsection{Modulation of the organic phase in SWCNT-based sensors}
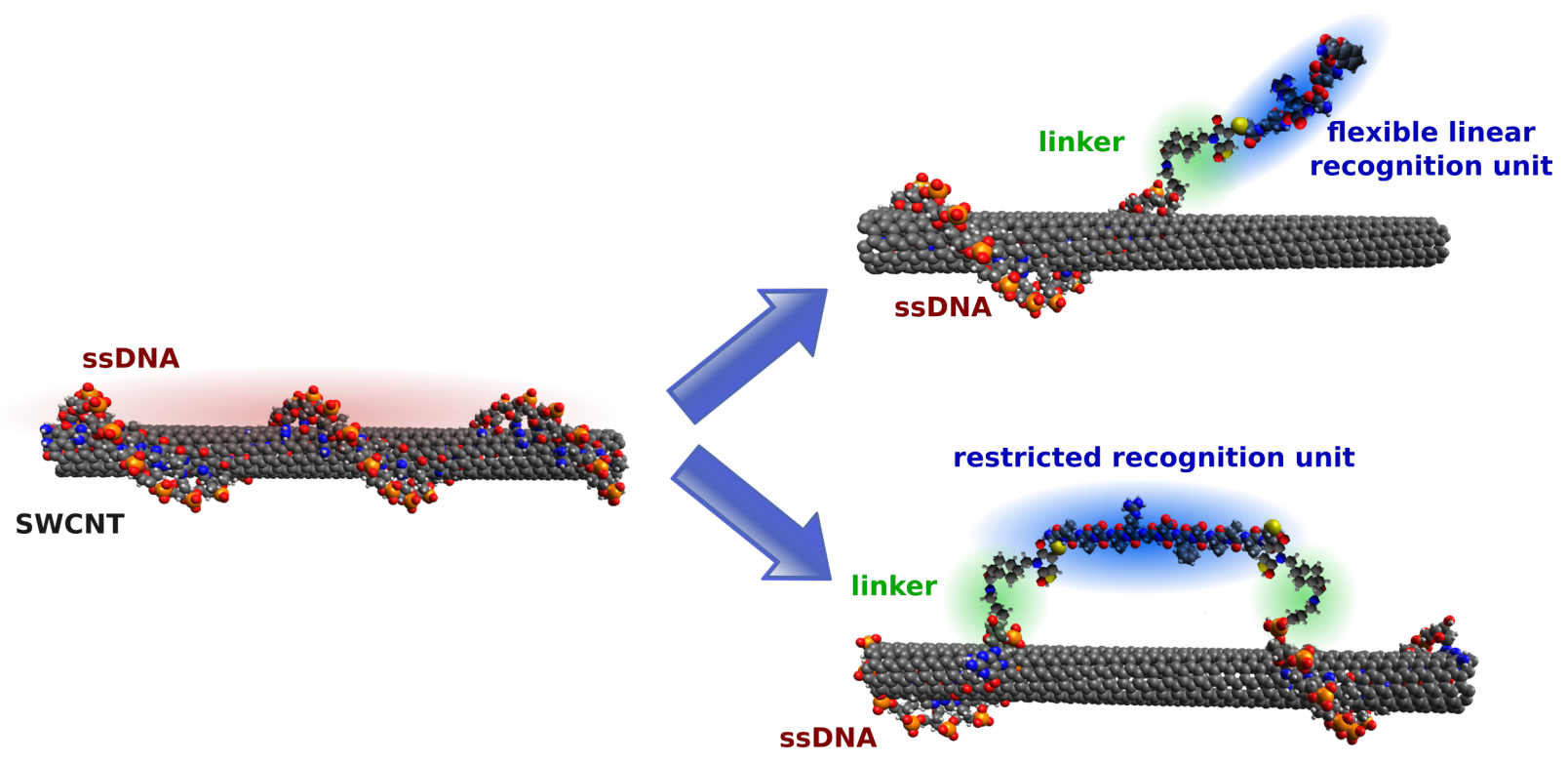

Figure 32: Process of modulation of the organic phase in SWCNT-based sensors, from a simple DNA to elaborated hybrid complexes. Schematics of a simple DNAbased wrapping and modular DNA-peptide motifs for specific targeting. Two possible geometries are shown: the recognition motif is anchored between two DNA sequences (bridge) or attached to the DNA sequence at one side (linear). The peptide serves as a recognition unit, while the DNA adsorbs onto the SWCNT surface and affects the exact peptide conformation.

As a proof of principle for future application, we focused on a peptide sequence wellestablished as a recognition unit: the integrin binding motif. There are different types of integrin, but most of them bind to the common short peptide sequence Arg-Gly-Asp (RGD). 
The conformational geometry of the RGD motif determines how strong and to which integrin type it binds.

Nanomaterials have been extensively modified with RGD motifs including gold and silver nanoparticles [257], 258], 259]. The first combination of RGD-chemistry and optical properties of SWCNTs was achieved by the group of Hongjie Dai [260], [261]. They investigated the biodistribution of integrin-positive tumors in mice by addressing the $\alpha_{v} \beta_{3}$ integrin via an RGD-unit attached to SWCNTs. For this purpose, SWCNTs were covalently functionalized with phospholipid-polyethyleneglycol (PL-PEG) of two different lengths: 100 and 300 nm. Afterwards, cyclic RGD-unit c(RGDyK) was conjugated to the PL-PEG/SWCNTs, thus providing a staining agent for $\alpha_{v} \beta_{3}$-positive tumors. In the next step, PL-PEG$\mathrm{c}(\mathrm{RGDyK}) / \mathrm{SWCNTs}$ were intravenously injected into the mice and followed by microPET scans over 24h. Both compounds showed SWCNTs enrichment in the liver, however the uptake of SWCNT conjugates with longer PEG-chains was lower. Different biodistribution of PL-PEG-c(RGDyK)/SWCNTs with various PEG lengths indicates a correlation between the chemical environment of the RGD-unit and the binding affinities of the RGD motif.

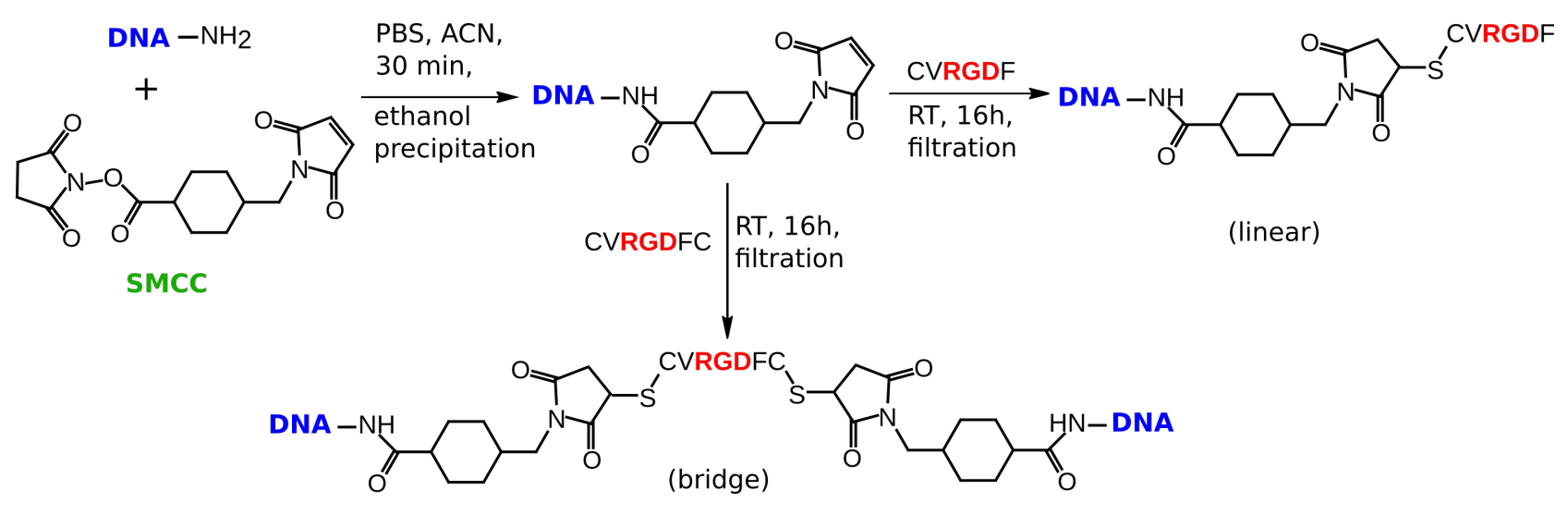

Figure 33: Conjugation strategies for ssDNA-peptide-ssDNA (bridge) and ssDNApeptide (linear) geometries.

So far, all these experiments used free conformation of the RGD motif. Instead, we present an approach that will confine the binding motif and thus control the binding affinities of RGD to the target (integrin). First, we conjugated amino-modified ssDNA to the heterofunctional linker (succinimidyl 4-(N-maleimidomethyl)cyclohexane-1-carboxylate, SMCC). In the second step, the peptide sequence including RGD-binding motif was conjugated to the linker-ssDNA. For a more detailed description of peptide synthesis and the conjugation strategy, we refer to Experimental Procedures 3.2.2.

Linear peptide hybrids with conformational freedom of recognition unit should have easier access into the integrin's binding pocket. At the same time, a linear conformation results 
in poor stability regarding enzymatic degradation and low selectivity for individual integrin types [191]. This drawback can be significantly improved by a prearranged conformation through cyclization or any other confinement of the binding motif. Our approach restricts the conformational freedom of the RGD motif. The basic idea of this approach is that the DNA determines the conformational freedom of the RGD motif and thus its binding affinity. To test this hypothesis we employ two strategies: (1) the RGD-sequence is conjugated to one end of a ssDNA strand (linear, ssDNA-RGD), or (2) the RGD-sequence is placed in between two flanking ssDNA strands (bridge, ssDNA-RGD-ssDNA), as shown in Figure 33.

\section{Characterisation of novel ssDNA-peptide/SWCNT hybrids}

As in the previous project, the samples were prepared by tip sonicating $(6,5)$ chirality enriched SWCNTs in a solution of the respective ssDNA-peptide macromolecules. Absorption spectra of these hybrids show typical $(6,5)$-SWCNTs $\mathrm{S}_{11}$ peaks in the nIR and $\mathrm{S}_{22}$ in the visible range. In comparison to $\mathrm{S}_{11}$ peaks of the ssDNA/SWCNTs from the first project (990 $\mathrm{nm})$, these peaks are red-shifted $(1050 \mathrm{~nm})$. The red-shift is often observed in other polymers in comparison to ssDNA [137]. Similarly, emission spectra reflect this shift in the nIR fluorescence. The observed emission intensity indicates that the synthesis approach leads to stable suspensions without aggregations and that the nIR fluorescence is preserved. We refer to Appendix 6 for absorption and emission spectra of all hybrids.

a

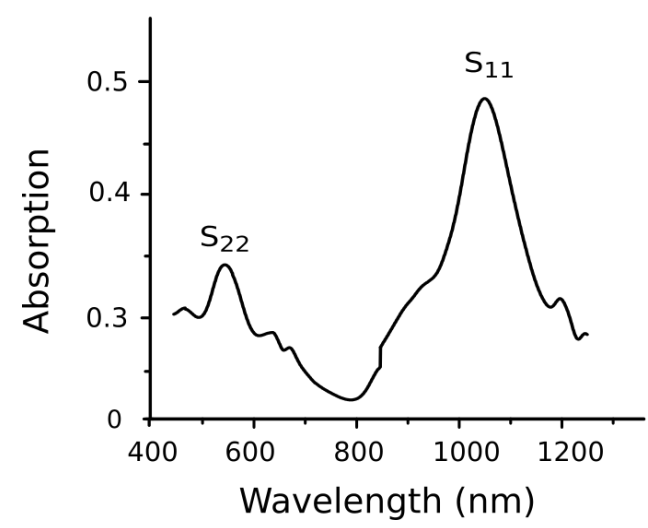

b

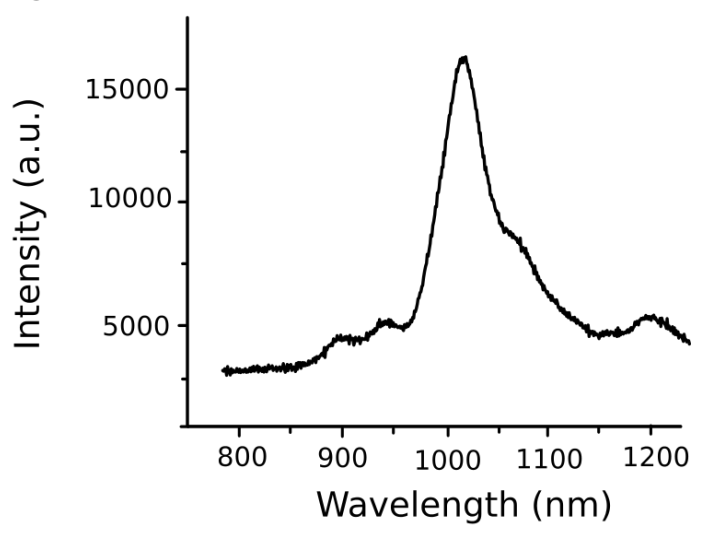

Figure 34: Absorption and emission spectra of ssDNA-peptide/SWCNT hybrids. (a) Typical absorption spectrum of ssDNA-peptide/SWCNT hybrids, here $(\mathrm{C})_{30^{-}}$ RGD/SWCNT. $\mathrm{S}_{11}$ and $\mathrm{S}_{22}$ denote optical transitions of SWCNTs. (b) Typical nIR fluorescence emission spectrum of ssDNA-peptide/SWCNT hybrids, here $(\mathrm{C})_{30^{-}}$ RGD/SWCNT. 


\subsubsection{Modulation of integrin affinity by changing the RGD-motif}

After the UV-Vis-nIR characterization we investigated how different ssDNApeptide/SWCNT hybrids affect RGD binding to integrins. For this purpose, we have developed an enzyme-linked immunosorbent binding assay (ELISA). This ELISA quantifies the binding between fibrinogen and soluble $\alpha_{I I b} \beta_{3}$ integrin. After adding RGD-containing polymer/SWCNTs into the system, they will compete with fibrinogen for the binding to $\alpha_{I I b} \beta_{3}$ integrin. Thus, we can quantify the binding affinity of various RGD-containing polymer/SWCNTs to the integrin. As mentioned in the theory section 2.2.4, $\alpha_{I I b} \beta_{3}$ integrin is prevalent on platelets and binds to the RGD-motif [179]. Figure 35 illustrates the schematics of the assay.

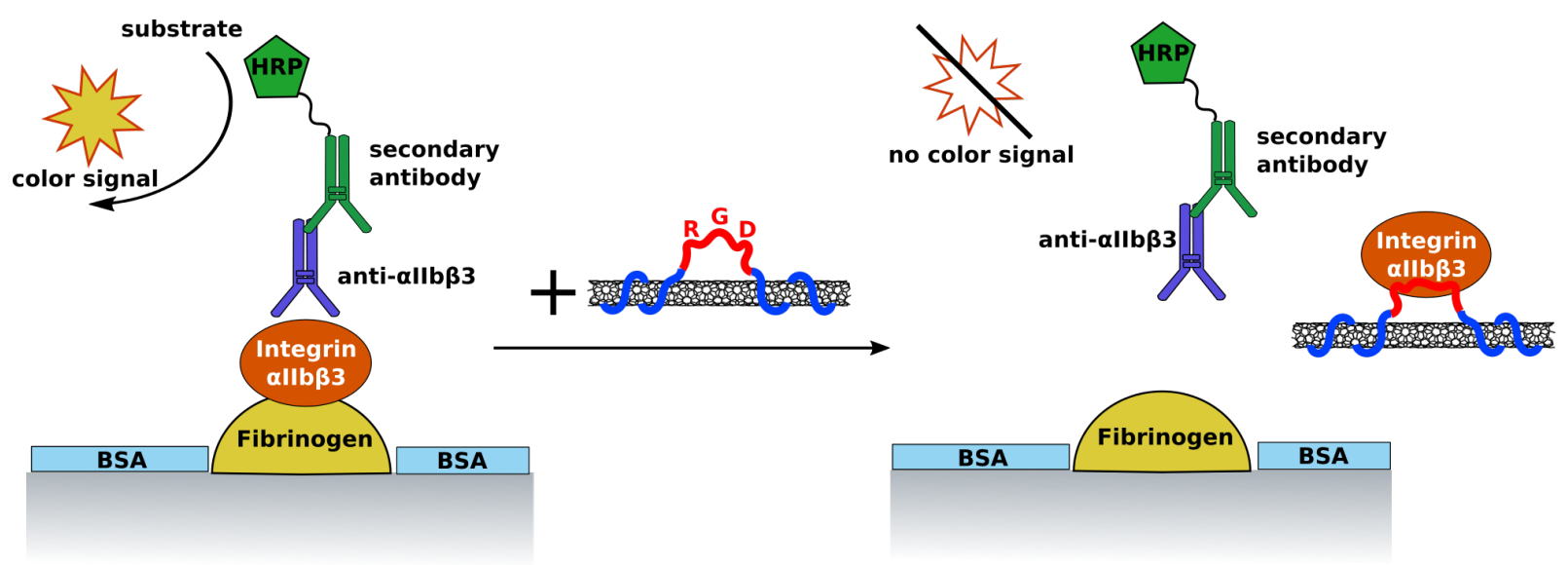

Figure 35: Inhibition of integrin by ssDNA-RGD/SWCNTs. Schematic of a binding assay developed to quantify the affinity between RGD-containing polymer/SWCNTs and soluble $\alpha_{I I b} \beta_{3}$ integrin. Without RGD-motifs, a bright color can be observed. The binding chain goes from fibrinogen to integrin, then to two antibodies, and ends with horseradish peroxidase (HRP) as the final block. After addition of a substrate, HRP forms yellow product that can be detected. Upon introduction of RGD-containing polymer/SWCNTs into the system, the binding chain is disrupted and the observed intensity decreases.

The sequence of this ELISA is as following: first, we coat the wells with fibrinogen that is known to bind integrin [177]. A sequence of integrin and two antibodies follows, with horseradish peroxidase (HRP) as the final block. After addition of a substrate, HRP forms yellow product that can be detected at $492 \mathrm{~nm}$. Upon introduction of RGD-containing polymer/SWCNTs into the system, it competes with fibrinogen for the free $\alpha_{I I b} \beta_{3}$ integrin. When ssDNA-RGD/SWCNT-integrin is washed out of the system, the binding chain is 
disrupted and the observed intensity decreases. For a detailed description of the ELISA procedure we refer to section 3.2 .4 .

To account for possible non-specific binding between ssDNA-peptide/SWCNTs and integrin, we used a second peptide sequence: Arg-Gly-Glu (RGE). The difference between RGD and RGE is just a slight change in the last amino acid: from aspartic acid (D) to glutamic acid (E). Glutamic acid has one carbon-carbon bond more and is therefore slightly longer than the aspartic acid. However, for its binding to integrin the change is essential. The RGE peptide sequence displays no binding affinity to the integrin binding pocket 188]. Therefore, ssDNA-RGE/SWCNTs hybrids as a control analyte help to exclude any unspecific bindings. Typical binding curves for ssDNA-RGD- and ssDNA-RGE/SWCNTs are shown in Fig. 36.
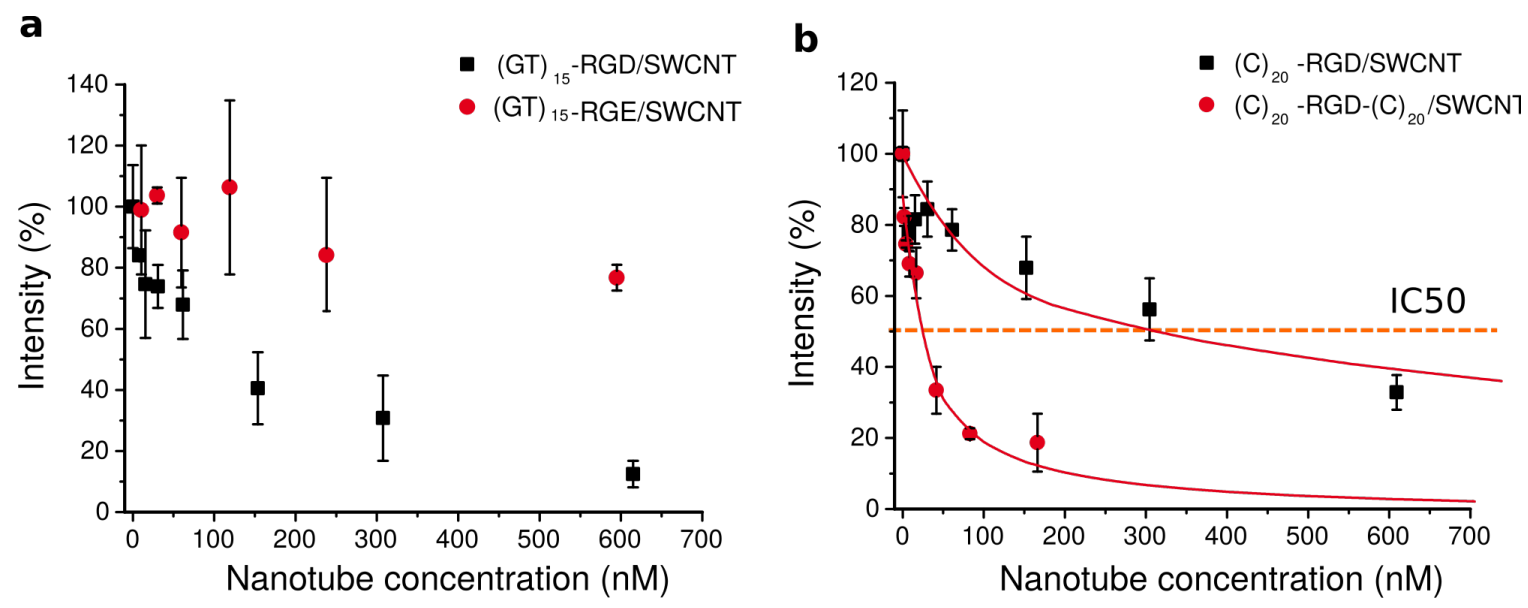

Figure 36: Typical inhibition curves for ssDNA-peptide/SWCNT hybrids. (a) Inhibition curves for $(\mathrm{GT})_{15}$-RGD/SWCNTs (black squares) and $(\mathrm{GT})_{15}$-RGE/SWCNTs (red circles). Intensities are normalized to the maximal value without ssDNApeptide/SWCNTs (100\% intensity) and the background signal of well-plates without integrin (0\% intensity). (b) A comparison of inhibition curves for linear $(\mathrm{C})_{20^{-}}$ RGD/SWCNTs (black squares) and bridged (C) 20 -RGD-(C) 20 /SWCNTs (red circles). Four parameter logistic function fits are shown as the red lines. Dashed orange line marks the $50 \%$ drop in the initial intensity (50\% inhibition, IC50). Error bars are standard errors $(n=2)$.

IC50 values were calculated using a 4-parameter logistic function to fit the experimental data:

$$
y=d+\frac{a-d}{1+\left(\frac{x}{c}\right)^{b}},
$$

with $a$ as the minimum value that can be obtained, $d$ as the maximum value, $c$ as the point 
of inflection and $b$ as Hill's slope of the curve (steepness). The rearranged equation allows to calculate the concentration of $x$ at the point of $y=50 \%$, thus determining IC50 values for ssDNA-peptide/SWCNTs hybrids. Figure 37 demonstrates color-coded IC50 values, with black as the lowest and white as the highest IC50 value.

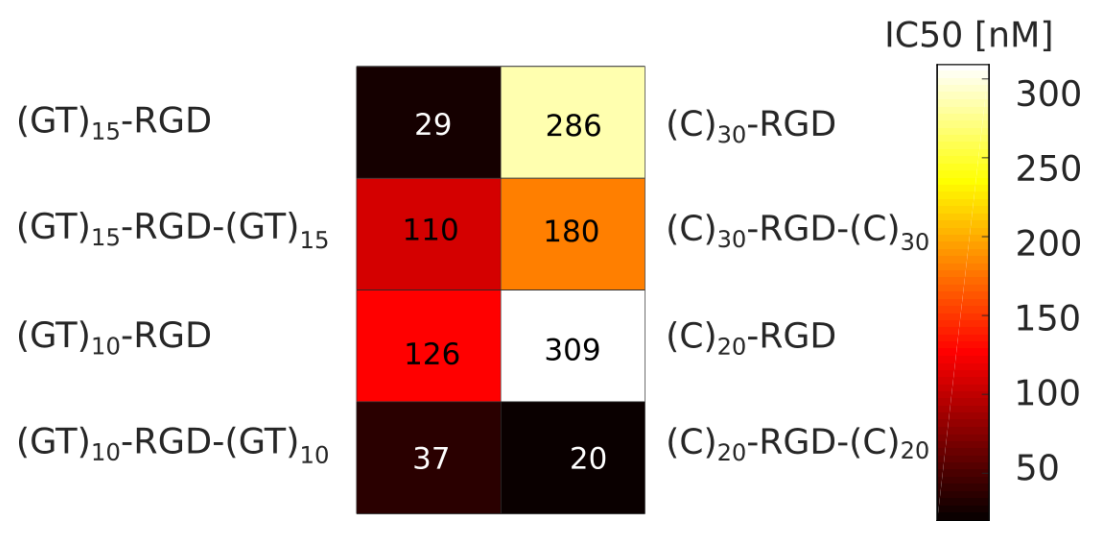

Figure 37: IC50 values of ssDNA-peptide/SWCNTs competitively binding to $\alpha_{I I b} \beta_{3}$ integrin. The values were calculated by logistic function fits from the ELISA assay, with black as the lowest and white as the highest IC50 value.

When comparing IC50 values for various sequences, there might be a tendency for hybrids containing poly-(GT) (sequences of repeating guanine and thymine nucleotides) to display higher affinity compared to poly(cytosine) containing hybrids. On the one hand, the IC50 values might be affected by varying affinity between different ssDNA sequences and SWCNTs. This binding affinity was measured in an AFM study by pulling various ssDNA sequences from the SWCNTs surface 138 . Based on the applied force, Iliafar et al. established following ranking for binding strength of different homopolymeres: $\operatorname{poly}(\mathrm{T})>\operatorname{poly}(\mathrm{A})>\operatorname{poly}(\mathrm{G})$ $\geq \operatorname{poly}(\mathrm{C})$, with values ranging from 11.3 to $7.58 k_{B} T$ per nucleotide. On the other hand, Jena et al. recorded fluorescence response of SWCNTs upon a oligonucleotide displacement and so investigated the affinity of poly (GT) to SWCNTs [262]. Their findings showed no correlation between the stability of a ssDNA/SWCNT complex and ssDNA length or SWCNT chirality. Instead they proposed that individual SWCNT chiralities have an enhanced affinity for a specific ssDNA sequence, e.g. $(\mathrm{GT})_{6}$ was identified as the recognition sequence with the highest affinity for $(8,6)$ SWCNTs.

In Fig. 37 no direct correlation of IC50 values with either ssDNA length or sequence is apparent. The lowest IC50 value was observed for bridged $(\mathrm{C})_{20}$-RGD- $(\mathrm{C})_{20} / \mathrm{SWCNT}(20$ $\mathrm{nM})$, while its linear equivalent showed the highest IC50 value (309 nM). In contrast, bridged $(\mathrm{GT})_{15}$-RGD-(GT) ${ }_{15} / \mathrm{SWCNT}$ (110 nM) had higher IC50 value than its linear counterpart $(29 \mathrm{nM})$. That suggests that all three parameters, ssDNA sequence, length and geometry (linear vs. bridged), modulate the affinity of ssDNA-peptide/SWCNTs to $\alpha_{I I b} \beta_{3}$ integrin. 


\subsubsection{Effective affinity of a single RGD motif}

To determine the origin of differences in IC50 values for various ssDNA-RGD/SWCNT hybrids, it is important to distinguish between affinity that results from a high number of weak binding RGD motifs or from a high binding affinity of a single favorable RGD-confinement. The number of adsorbed ssDNA-peptide macromolecules and thus the number of RGD motifs per SWCNT could vary for different sequences and ssDNA lengths. To determine the stoichiometry of RGD motifs per SWCNT, we will first calculate the SWCNT concentration, and then present a novel technique to determine ssDNA surface coverage around SWCNTs.

\section{SWCNT concentration}

Nanotube concentration for all ssDNA-peptide/SWCNT hybrids was calculated based on the $\mathrm{S}_{11}$ absorption peak of SWCNTs. As the molar extinction coefficient for SWCNTs we used $\varepsilon_{S W C N T}=67001 /(\mathrm{Mcm})$ calculated by Weismann et al. [263]. First, we have to determine the amount of C-atoms in a SWCNT. For geometrical considerations we used an estimation of a $350 \mathrm{~nm}$ long SWCNT (based on previous AFM measurements [57]).

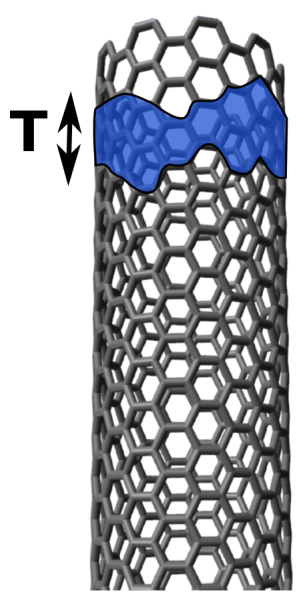

$(10,10)$

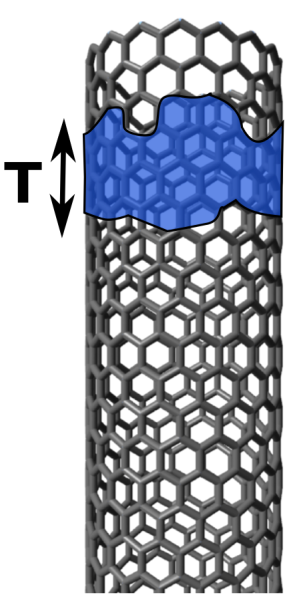

$(17,0)$

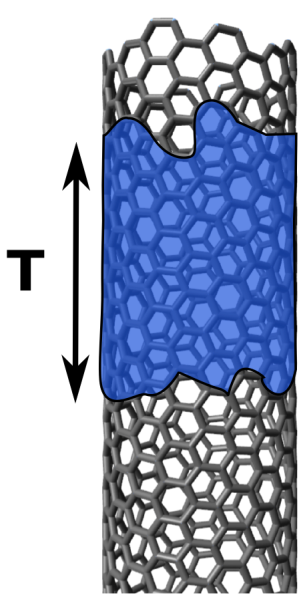

$(12,8)$

Figure 38: Unit cell length in SWCNTs. The unit cells of three different chiralities $(10,10)$, $(17,0)$ and $(12,8)$ are schematically demonstrated, with the translational length $\mathbf{T}$ of a unit cell highlighted in blue. From left to right $\mathbf{T}$ varies as following: For $(10,10)$ SWCNTs the length of $\mathbf{T}$ spans $2.46 \AA$ and contains 40 carbon atoms, for $(17,0)$ SWCNTs $4.26 \AA$ and 68 carbon atoms, and for $(12,8)$-SWCNTs $18.58 \AA$ with 304 carbon atoms. 
The unit cell of a SWCNT depends strongly on its chirality. The translational length $\mathbf{T}$ of a unit cell can very from 4 up to $85 \AA$. Figure 38 illustrates different translational lengths $\mathbf{T}$ for various chiralities. The number $q$ of graphene hexagons in a SWCNT unit cell $n_{c}$ is given by the ratio of cylinder surface area $S_{t}$ to the area $S_{g}$ of the hexagonal graphene unit cell [81]:

$$
q=\frac{S_{t}}{S_{g}}=\frac{2\left(n^{2}+n \cdot m+m^{2}\right)}{n_{l} R}
$$

A graphene unit cell contains 2 C-atoms, thus the number of carbon atoms in a unit cell is given by:

$$
n_{c}=2 q=\frac{4\left(n^{2}+n \cdot m+m^{2}\right)}{n_{l} R}
$$

with $n_{l}$ as the greatest common divisor of $(\mathrm{n}, \mathrm{m}) . R$ is 3 if $(n-m) / 3$ is an integer, otherwise $R=1$. For $(6,5)$-SWCNTs we get $n_{l}=1$ and $R=1$. Hence, the product of the divisor is 1 , so that we can simplify the equation to:

$$
n_{c}=2 q=4\left(6^{2}+6 \cdot 5+5^{2}\right)
$$

For $(6,5)-S W C N T s$, a unit cell has 364 carbon atoms. The translational length $\mathbf{T}$ of a unit cell is given by:

$$
T=\frac{\sqrt{3} L}{n_{l}}
$$

with $L$ being the circumference of a SWCNT:

$$
L=\left|C_{v}\right|=a \sqrt{n^{2}+n \cdot m+m^{2}}
$$

For $(6,5)-S W C N T$ s this results in the translational length $\mathbf{T}$ of $4.06 \mathrm{~nm}$. Thus, in a 350 $\mathrm{nm}$ long SWCNT, we have 86.5 times the length of the unit cell, and the number of carbon atoms is calculated to 31486 atoms. 


\section{DNA stoichiometry in ssDNA/SWCNT complexes}

There are no well-established methods to determine DNA stoichiometry in ssDNA/SWCNT complexes and thus very little experimental data. The technique of solvatochromic shifts employed in the previous section provides only a percentage of polymer coverage, while the exact amount of ssDNA is unknown 243. Schöppler et al. used labeled ssDNA (ssDNAFAM) to calculate the ssDNA concentration from a decrease in the fluorescence lifetimes of ssDNA-FAM/SWCNT complexes in comparison to free ssDNA-FAM conjugates [264]. The exact amount of quenching induced by ssDNA-FAM/SWCNT was assumed without experimental support. The DNA concentrations from the fluorescence lifetime calculations were then combined with solvatochromic frequency shifts of $\mathrm{S}_{11}$ for ssDNA/SWCNTs and resulted in a ssDNA surface coverage of $\approx 20 \%$. From their estimation the helical pitch of ssDNA around SWCNTs is $8-10 \mathrm{~nm}$ and is restricted by both steric and electrostatic interactions, and the flexibility of the DNA backbone. Based on this calculation, a $(\mathrm{GT})_{16}$ ssDNA would need $16-20 \mathrm{~nm}$ on a SWCNT surface.

A study by Campbell et al. observed a regular pattern of ssDNA on the surface of ssDNA/SWCNT [265]. With atomic force microscopy they showed the pattern to be independent of the length and sequence of the ssDNA and suggested that the pitch of the ssDNA wrapping around SWCNT is $14 \mathrm{~nm}$.
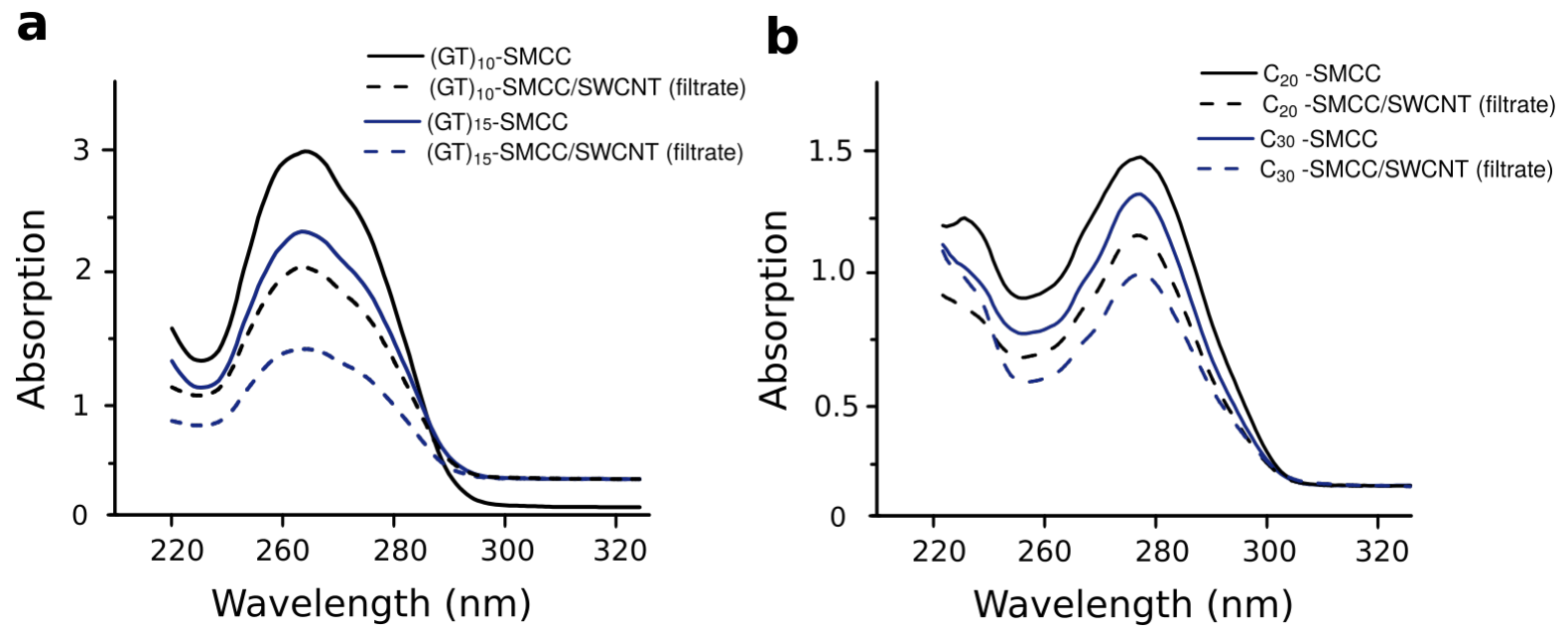

Figure 39: DNA concentration determined by UV-Vis spectroscopy. Spectra show absorption of the initial ssDNA solution (solid line) and absorption of the residual ssDNA in the filtrate of tip-sonicated and filtrated ssDNA/SWCNT samples (dashed line) for (a) poly(GT) and, (b) poly(cytosine) sequences. 
In our experiments, we determined the amount of ssDNA by collecting two UV-Vis spectra: (1) absorption of the initial ssDNA solution and (2) absorption of the residual ssDNA in the filtrate of tip-sonicated and filtrated ssDNA/SWCNT sample, as shown in Figure 39. The difference in these two ssDNA concentrations is assumed to be the ssDNA amount that adsorbed onto the SWCNT surface. A small ssDNA percentage might be detectable in the pellet after the centrifugation step. This would be a small systematic error that should not affect the trend of the data. Fig. 40 shows the number of different ssDNA-peptide units per single SWCNT.

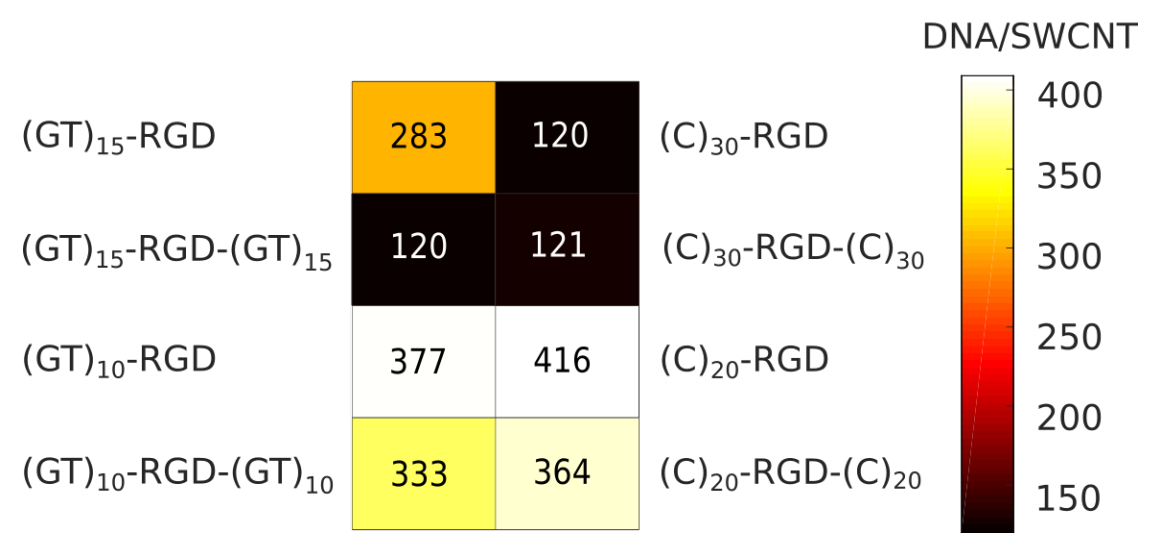

Figure 40: Stoichiometry of ssDNA in ssDNA/SWCNT complexes. Calculated by assuming a $350 \mathrm{~nm}$ long SWCNT and color-coded with black as the lowest and white as the highest value.

The stoichiometry of RGD-motifs show a 4-fold difference in their accommodation on SWCNTs. As expected, there is a tendency that longer ssDNA-peptide sequences occupy more space, so that fewer RGD motifs are present on these SWCNTs (e.g. 120 RGD-units on (C) 30 -RGD/SWCNT vs. 416 RGD-units on (C) 20 -RGD/SWCNT). It is also possible that shorter ssDNA sequences bind more weakly to SWCNTs than longer ssDNA sequences. Another parameter to consider is the geometry of ssDNA on the SWCNT. For longer ssDNA sequences a helix is the most probable conformation, while shorter ssDNA sequences are more likely to form different, more complex conformations [266]. These more complex geometries might render the RGD-motif less exposed to the solution and consequently less accessible to integrin binding. 


\section{Effective IC values}

Based on both ssDNA and SWCNT calculations, we can determine the effective IC50 values for each ssDNA-peptide hybrid by multiplying 'normal' IC50 values with the number of RGD-motifs per SWCNT (Fig. 37).

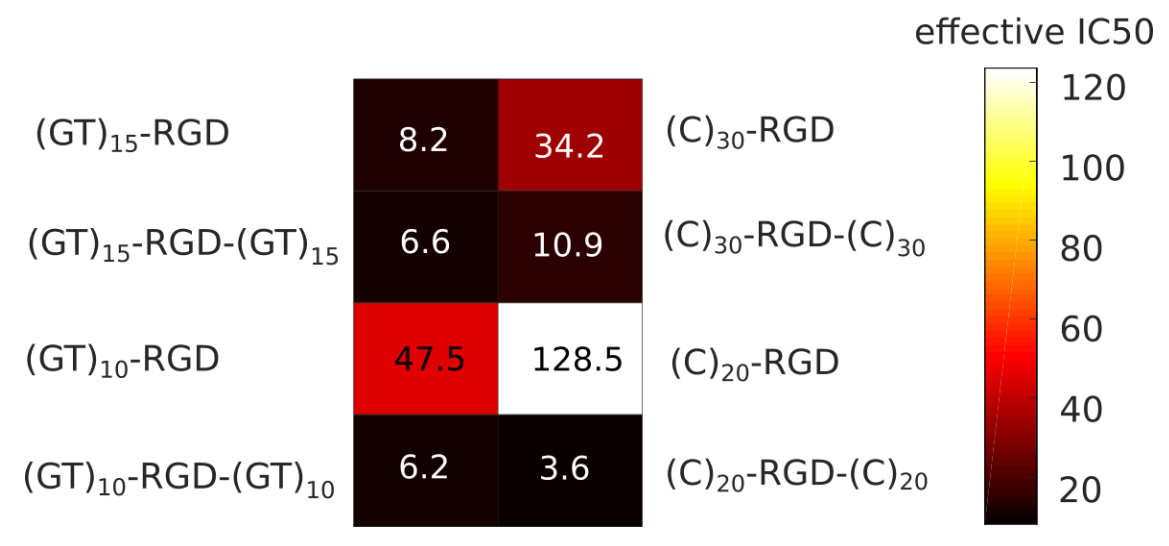

Figure 41: Effective IC50 values of competitive ssDNA-peptide/SWCNTs binding to $\alpha_{I I b} \beta_{3}$ integrin. Effective IC50 obtained by multiplying 'normal' IC50 values with the number of RGD-motifs per single nanotube, color-coded with black as lowest and white as highest values.

Interestingly, there are some variation between IC50 and normalized IC50 values. When one focuses on the affinity of the both $(\mathrm{C})_{30}-\mathrm{RGD} / \mathrm{SWCNT}$ and $(\mathrm{C})_{20}$-RGD/SWCNT complexes as a whole, the affinity is low in both cases (IC50 $=286 \mathrm{nM}$ vs $309 \mathrm{nM}$, respectively). However, at a single RGD-motif level, IC50 of $(\mathrm{C})_{30}$ hybrid is 4-time lower than that of $(\mathrm{C})_{20}$ (normalized IC50 $=34.2$ vs 128.7 ). Whereas in ssDNA sequences containing guaninethymine, the binding affinity seems unaffected by the length of ssDNA sequence or the number of single RGD units per nanotube. Previous IC50 values show a difference in factor 3 between $(\mathrm{GT})_{15}$-RGD- $(\mathrm{GT})_{15} / \mathrm{SWCNT}$ and its $(\mathrm{GT})_{10}$ equivalent, however on the single motif level their IC50 values are almost the same (6.6. and 6.2).

Interestingly, while the inhibition pattern changes, the SWCNT-hybrid with the lowest IC50 value stays the same. This result suggests that most of the affinity can be contributed to the conformational aspects and not the absolute numbers of RGD motifs. 


\subsubsection{Cell adhesion on ssDNA-RGD/SWCNT hybrids}
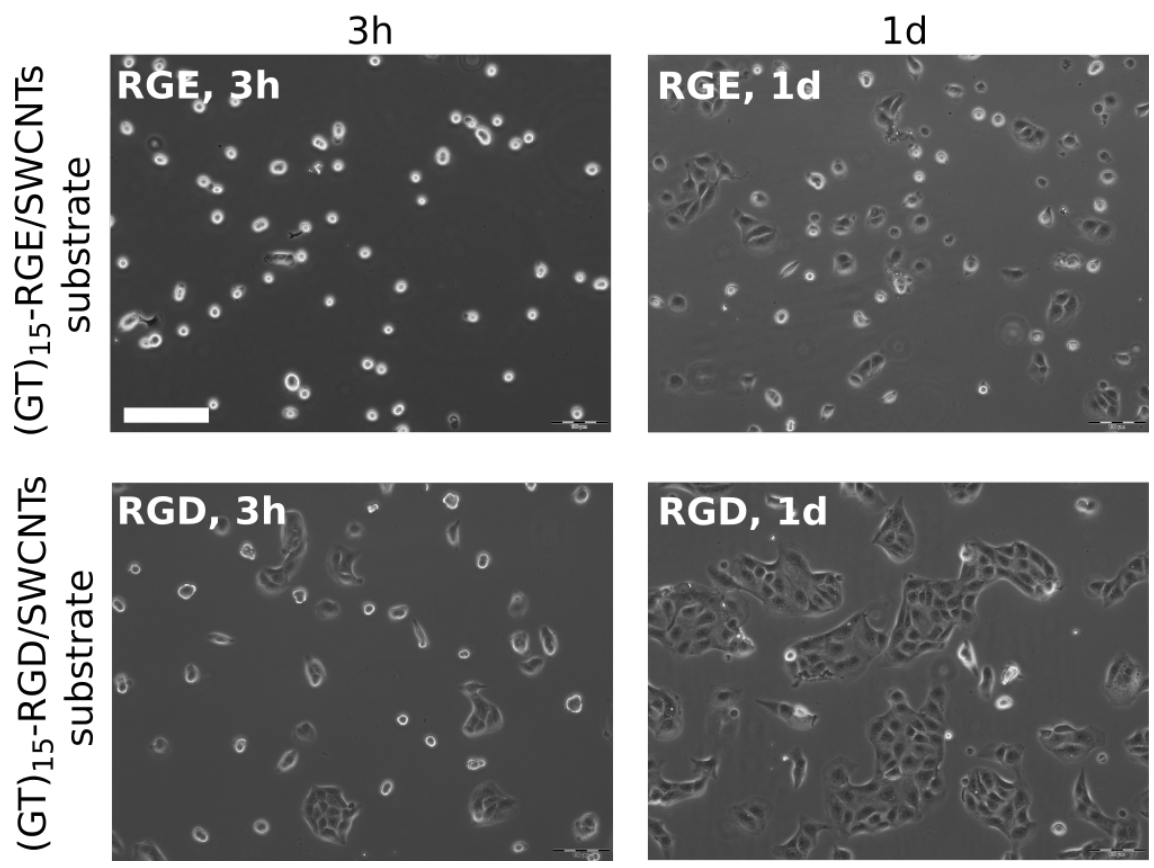

Figure 42: Adhesion of MDCKII cells on ssDNA-RGD/SWCNT hybrids. Phase contrast images of MDCKII cells spread on a glass surface coated with $(\mathrm{GT})_{15^{-}}$ RGD/SWCNTs and (GT) ${ }_{15}$-RGE/SWCNTs after 3 hours and 1 day (scale bar is $100 \mu \mathrm{m}$ ). Changes in their morphology (flat vs round) indicate the adhesion status of MDCKII cells.

In the next step, we investigated if ssDNA-RGD/SWCNT hybrids are functional after immobilization on the surface and how they affect integrin mediated adhesion. For that purpose we cultivated MDCKII cells, a well-established epithelial cell line that expresses integrins [267], 268]. Changes in their morphology indicate their adhesion status. Round morphology can be observed for not sufficiently adherent cells, while flattened morphology is representative of well spread cells. Phase contrast images of MDCKII cells cultivated for 3h showed higher total cell numbers and stronger adhesion for surfaces coated with $(\mathrm{GT})_{15^{-}}$ RGD/SWCNTs than with $(\mathrm{GT})_{15}$-RGE/SWCNTs, as can be seen in Figure 42 . Overall, we compared four surface types: glass, $(\mathrm{GT})_{15} / \mathrm{SWCNT}$ coated glass, $(\mathrm{GT})_{15}$-RGE/SWCNTs coated glass and $(\mathrm{GT})_{15}$-RGD/SWCNTs coated glass. Figure 43 shows the percentage of adherent cells and the total number of cells after 3 hours and 1 day (all values were normalized to the bare glass surfaces to account for unspecific adhesion of MDCKII to glass). 

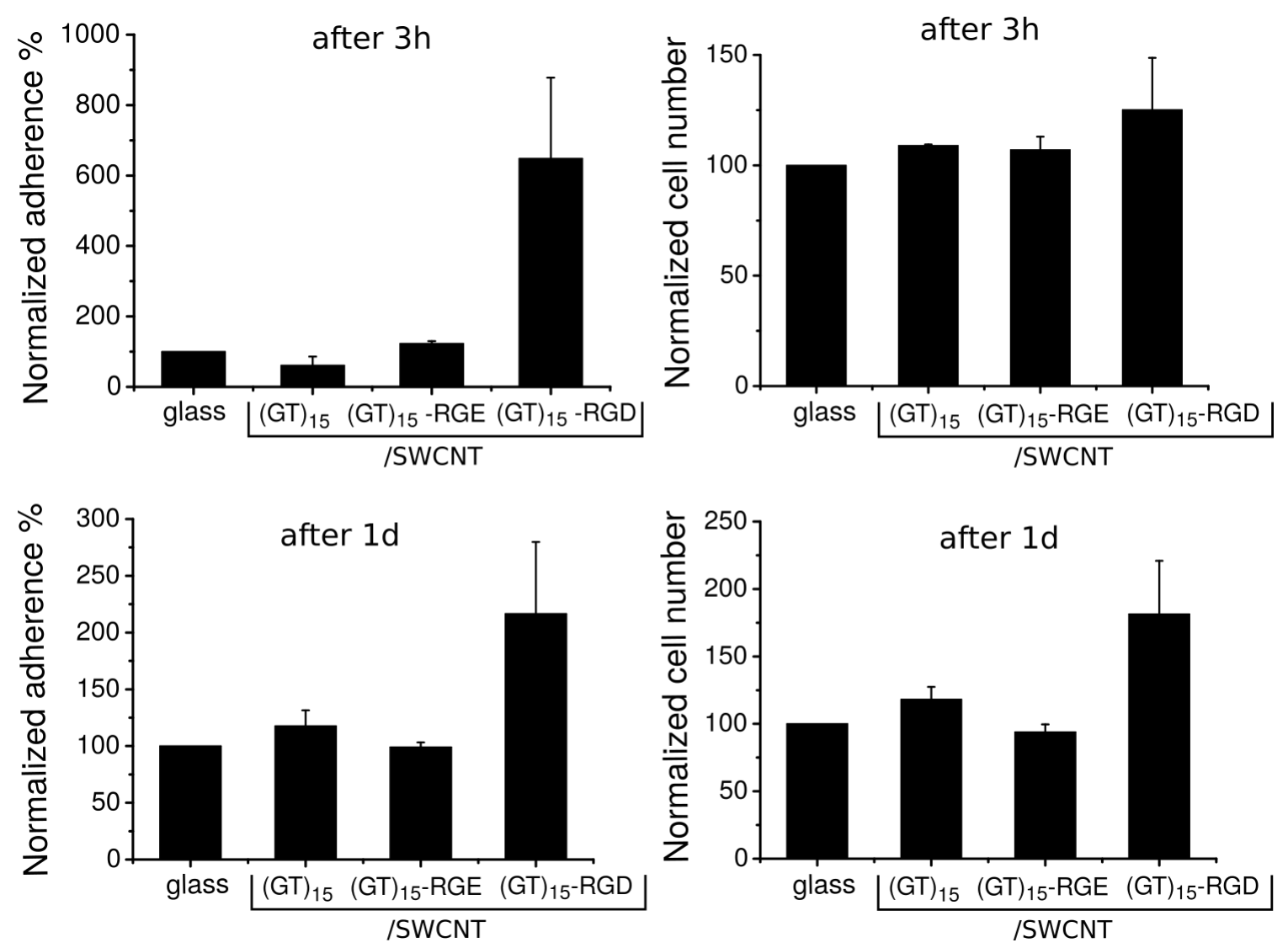

Figure 43: Adhesion of MDCKII cells seeded on ssDNA-RGD/SWCNT hybrids normalized to the values on the glass surface. Development of cells on four varying substrates: glass, $(\mathrm{GT})_{15} / \mathrm{SWCNTs}$ coated glass, $(\mathrm{GT})_{15}$-RGE/SWCNTs coated glass and $(\mathrm{GT})_{15}$-RGD/SWCNTs coated glass. Graphs show percentage of normalized adherent cells and normalized total number of cells 3 hours and 1 day after seeding on substrates (the values are normalized to the values on glass surface, error bars are standard errors, $\mathrm{n}=2$ ).

MDCKII cells clearly preferred the $(\mathrm{GT})_{15}$-RGD/SWCNTs coated glass over all other variations, as shown by the increased numbers of total cells, as well as the higher percentage of adherent cells. After 3 hours, 6-times more adherent cells were observed for $(\mathrm{GT})_{15^{-}}$ RGD/SWCNTs coating compared to $(\mathrm{GT})_{15}$-RGE/SWCNTs (1.2) or ssDNA/SWCNT (0.6) (with glass surface being 1.0). After 1 day the differences are starting to level out, but the total number of cells were still higher for $(\mathrm{GT})_{15}$-RGD/SWCNTs compared to the RGE equivalent. Detailed adhesion data for four different ssDNA-RGD/SWCNTs are presented in Appendix 6. Interestingly, the differences in adhesion of MDCKII cells on various ssDNARGD/SWCNTs with different ssDNA sequences were negligible. 


\subsubsection{Labeling of integrin on human blood platelets in the nIR}

a
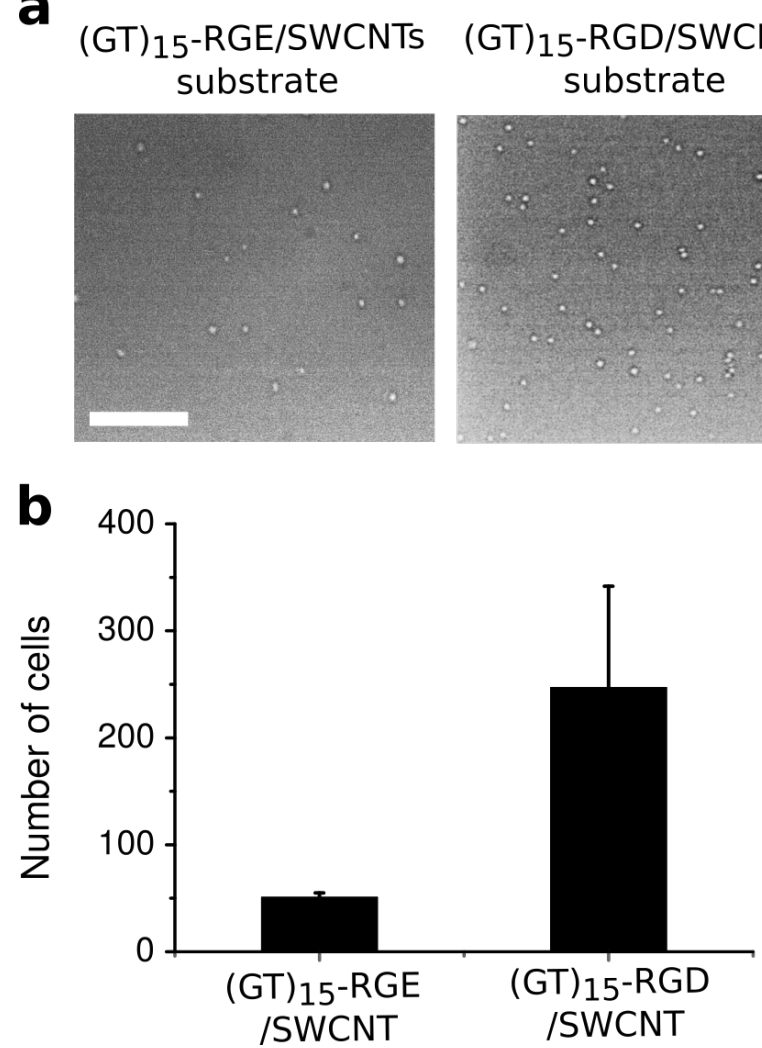

\section{Bright field image}

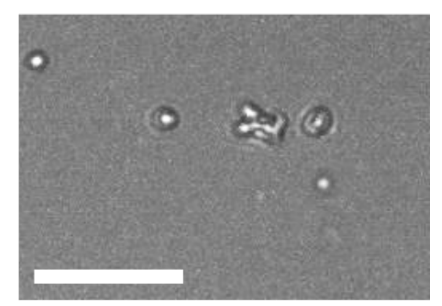

nIR image

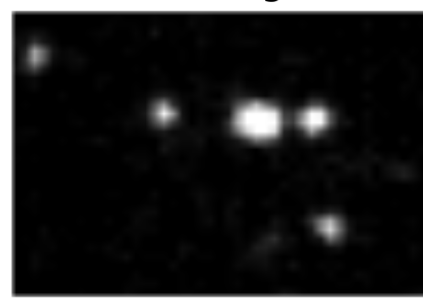

Overlay

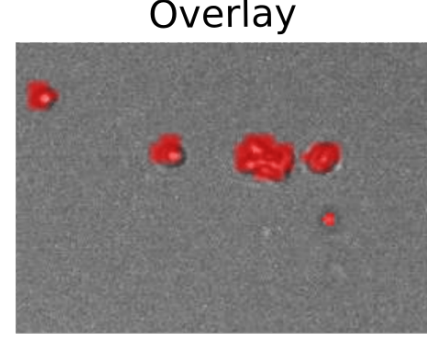

Figure 44: Near infrared labeling of $\alpha_{I I b} \beta_{3}$ integrins on human blood platelets with ssDNA-peptide/SWCNT hybrids. (a) Bright field images of platelets spread on surface with $(\mathrm{GT})_{15}$-RGD/SWCNTs and $(\mathrm{GT})_{15}$-RGE/SWCNTs coating after 1 hour (scale bar is $40 \mu \mathrm{m}$ ), (b) Total numbers of cells adherent on both substrates after 1h incubation on ssDNA-peptide/SWCNTs surfaces: (GT) ${ }_{15}$-RGD coated glass and its RGE equivalent (error bars are standard errors, $\mathrm{n}=2$ ), (c) From top to bottom: bright field image of platelets stained with $(\mathrm{GT})_{15}$-RGD/SWCNTs, nIR image of $(\mathrm{GT})_{15}$-RGD/SWCNTs, and an overlay of bright field and nIR images to show the co-localization of staining on platelets (scale bar is $25 \mu \mathrm{m}$ ).

MDCKII cell line is often used for morphological studies, thus we could easily monitor the adhesion status of the cells. Yet MDCKII are also known to expresses several $\beta_{1}$ integrin types, such as $\alpha_{2} \beta_{1}, \alpha_{3} \beta_{1}$, as well as $\alpha_{v} \beta_{3}$ and $\alpha_{6} \beta_{4}$ integrins [267]. Therefore, the general adhesion properties to various integrin types can be easily studied and evaluated using MDCKII cells. However, for the ELISA binding assay we used integrin that was extracted from human platelets. $\alpha_{I I b} \beta_{3}$ is the major integrin type to be found on the surface of these blood cells [179]. Therefore, to specifically address the $\alpha_{I I b} \beta_{3}$ integrin, we seeded platelets 
on surfaces coated with ssDNA-peptide/SWCNTs hybrids. As shown in Figure 44, platelets clearly preferred the $(\mathrm{GT})_{15}$-RGD/SWCNTs coated glass, as demonstrated in an increased numbers of total cells: $51 \pm 4$ per picture in case of $(\mathrm{GT})_{15}$-RGE/SWCNTs versus $247 \pm 95$ per picture in case of $(\mathrm{GT})_{15}$-RGD/SWCNTs.

The use of integrin coating to facilitate the adhesion of cells has been shown in several model systems including endothelial cells, neutrophils or cancer cells [269], [270], 271]. However, additionally to being a scaffold for RGD confinement, the ssDNA-peptide/SWCNT hybrids introduce a unique nIR fluorophores into the system. Therefore, we used $(\mathrm{GT})_{15^{-}}$ RGD/SWCNTs hybrids to selectively label $\alpha_{I I b} \beta_{3}$ integrin on freshly isolated human platelets. Figure $44 \mathrm{c}$ shows a phase contrast image of isolated human blood platelets stained with $(\mathrm{GT})_{15}$-RGD/SWCNTs (the labels are not visible in bright field). An overlay of phase contrast image of platelets with the near infrared image of $(\mathrm{GT})_{15}$-RGD/SWCNTs shows distinct co-localization. Such staining would allow to label specific integrin types in the nIR.

\subsubsection{Conclusion}

In this chapter, we combined a well-established peptide recognition unit, Arg-Gly-Asp (RGD), with various ssDNA sequences and the nIR fluorophore SWCNT. In a modular sensor approach the binding affinity of the recognition unit (RGD) to its target (integrin) was tuned via small changes of one of the sensor components (ssDNA length and sequence). After synthesis and conjugation, we have quantified how different ssDNA-peptide/SWCNT hybrids affect the binding of the RGD-motif to $\alpha_{I I b} \beta_{3}$ integrin via an enzyme-linked immunosorbent binding assay (ELISA). IC50 values (50\% inhibition) for the different hybrids could be tuned from 20 to $309 \mathrm{nM}$. The lowest IC50 value was observed for bridged $(\mathrm{C})_{20}$-RGD$(\mathrm{C})_{20} / \mathrm{SWCNT}$ (20 nM); its linear equivalent was the one with the highest IC50 value (309 $\mathrm{nM})$. In contrast, another bridged hybrid (GT) ${ }_{15}$-RGD-(GT) ${ }_{15} / \mathrm{SWCNT}(110 \mathrm{nM})$ showed higher IC50 value than its linear counterpart $(\mathrm{GT})_{15}$-RGD/SWCNT (29 nM). From an absent trend we concluded that all three parameters, ssDNA sequence, length and geometry (linear vs. bridged), modulate the affinity of ssDNA-RGD/SWCNTs in a complex manner. To determine the origin of differences in IC50 values of various hybrids, we calculated the number of adsorbed ssDNA-peptide macromolecules and thus the number of RGD motifs per SWCNT. The stoichiometry of RGD-motifs demonstrated a 4-fold difference in the number of RGD-motifs: from 120 RGD-units for (C) $30^{-R G D / S W C N T ~ t o ~} 415$ RGD-units for (C) ${ }_{29^{-}}$ RGD/SWCNT. As expected, our results confirmed that longer ssDNA-peptide sequences occupy more space and thus accommodate fewer RGD motifs per nanotube. Effective IC50 values were obtained by multiplying 'normal' IC50 values with the number of RGD-motifs per single nanotube. It was shown that while the inhibition pattern changed, the SWCNThybrid with the lowest IC50 value remained the same. This finding indicates that the major 
contribution to the affinity originates from the conformational aspects and not the number of RGD motifs.

We also demonstrated that the RGD-motif is functional after immobilization on the surface by analyzing the morphology of cell adherence on surfaces coated with ssDNARGD/SWCNTs. By incorporating SWCNTs into the sensors we created not only a possibility for scaffolding but also added a unique nIR fluorophore to the investigated system. Therefore, we applied this characteristic to label human platelet cells with ssDNA-RGD/SWCNT hybrids. This combination of SWCNTs fluorescence with the high selectivity of RGD-motifs offers new opportunities for specific integrin labeling in the nIR. The modular approach can be adapted for other target molecules, e.g. neurotransmitter GABA. On the one hand, a sensor design that is not rational makes creating a specific sensor difficult and time-consuming. On the other hand, the ability to tune and amplify the binding affinity of a recognition unit via smallest changes opens new possibilities that can be explored in a high throughput sensor screening. 


\subsection{Egyptian Blue as novel nIR fluorophore}

In previous chapters, we focused on SWCNTs as nIR fluorophore building blocks for sensor applications. Here, we will explore another nIR fluorophore beyond SWCNTs: Egyptian Blue $\left(\mathrm{CaCuSi}_{4} \mathrm{O}_{10}\right)$. Egyptian Blue (EB) is a calcium copper silicate with promising photophysical properties, such as high quantum yield, long fluorescent lifetime in the range of microseconds and emission in the $\mathrm{nIR}$ region $\left(\lambda_{e x}=620 \mathrm{~nm}, \lambda_{e m}=930 \mathrm{~nm}\right.$, as shown in Fig. 45).

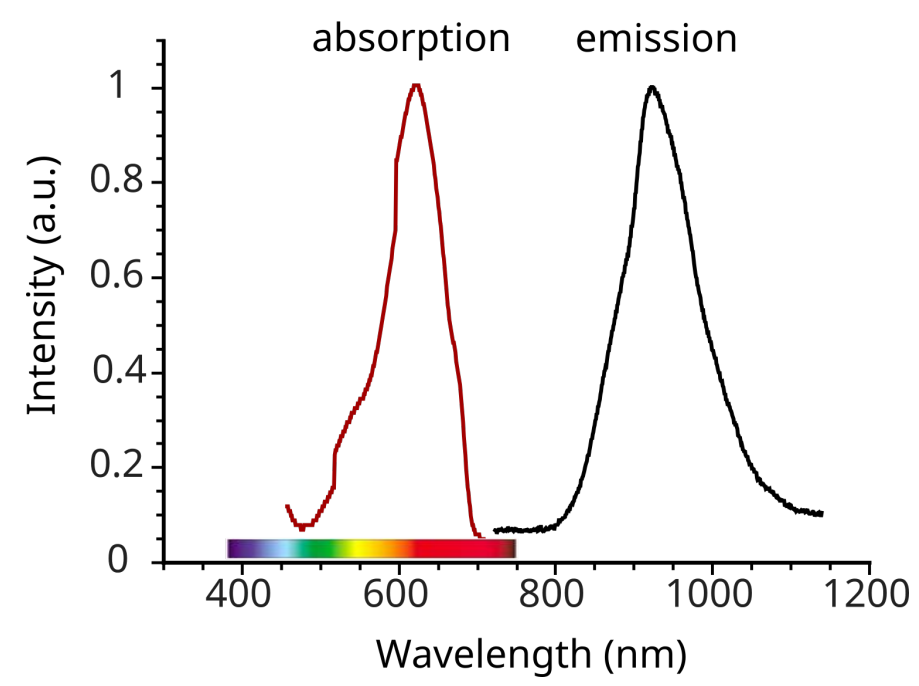

Figure 45: Normalized absorption and emission spectra of Egyptian Blue.

\subsubsection{Investigation of Egyptian Blue nanosheets by atomic force microscopy (AFM)}

The size of the sensor should be comparable to that of the investigated system. Furthermore, a three-dimensional bulk material has less potential for sensing, since small changes on the surface (e.g. a binding event) do not necessarily change photophysical properties of the whole material. Additionally, aqueous EB nanosheet dispersions could provide a new possibility to process the material in colloidal form, e.g. via functionalization. Therefore, the first step to the new sensors would be to create EB particles in nanodimensions. Figure 46 illustrates the size reduction of the EB powder sample via tip sonication.

We tried three approaches to reduce the particle size of EB samples: (1) It was reported that stirring $\mathrm{EB}$ in hot water $\left(85^{\circ} \mathrm{C}\right)$ at $400 \mathrm{rpm}$ for two weeks will result in thin $(2-3$ $\mathrm{nm})$ nanosheet products with lateral expansion ranging from hundred nanometers to several microns (measured by TEM) 272]. Since in the reported preparation was a filtration step (with $0.4 \mu \mathrm{m}$ size exclusion filters) the lateral dimensions of several $\mu \mathrm{m}$ might be slightly overestimated. (2) Han Blue was reported to be unresponsive to the heat exfoliation method. 
Instead it required sonication to achieve similar results. Therefore, the second possibility would be to use tip sonication, similar to our SWCNT dispersion preparation. (3) And finally, the third pathway was based on a graphene exfoliation technique. Here, EB powder would first stick to the adhesive tape A and then be peeled off by the tape B. More details on the exfoliation methods can be found in Appendix 6 .

a

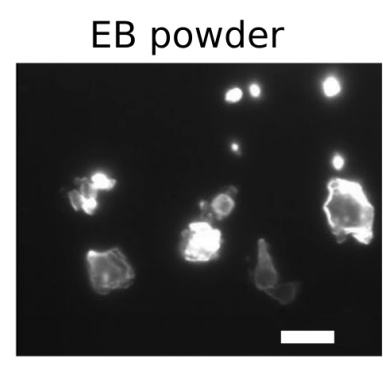

b

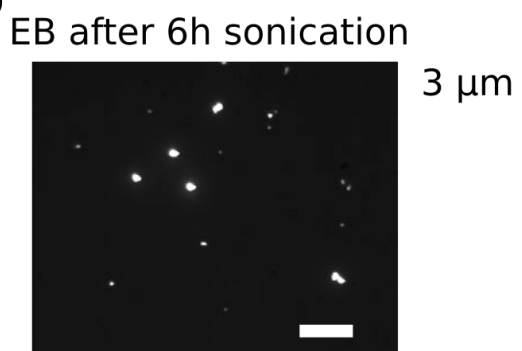

C

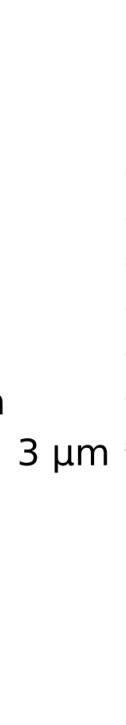

Figure 46: nIR photoluminescence images of EB particles. (a) Near-IR image of an unprocessed powder sample of commercial EB. The scale bar is $50 \mu \mathrm{m}$. (b) Near-IR image of EB particles after $6 \mathrm{~h}$ sonication. The scale bar is $50 \mu \mathrm{m}$, (c) 3D intensity profile of an EB nanoparticle from the image (b), color-coded with red $=255$ and blue $=0$ (the laser intensity is $50 \mathrm{~mW}$ in all images, $\lambda_{e x}=560 \mathrm{~nm}$ ).

After identifying the tip sonication technique as the most promising method for producing small and homogeneous particles (average area $\approx 18 \mu \mathrm{m}^{2}$ ), we prepared an EB sample with $6 \mathrm{~h}$ sonication in isopropanol. After the sonication, isopropanol was evaporated overnight and the dry powder was used in all following experiments. As shown in the 3D intensity profile of a single nanoparticle (Fig. 46c), the light microscopy resolution limit prevents us from resolving the exact size of the particle. Therefore, we conducted topographic atomic force microscopy (AFM) on the sub-micron structures of our samples (Fig. $47 \mathrm{a}-\mathrm{b}$ ). 

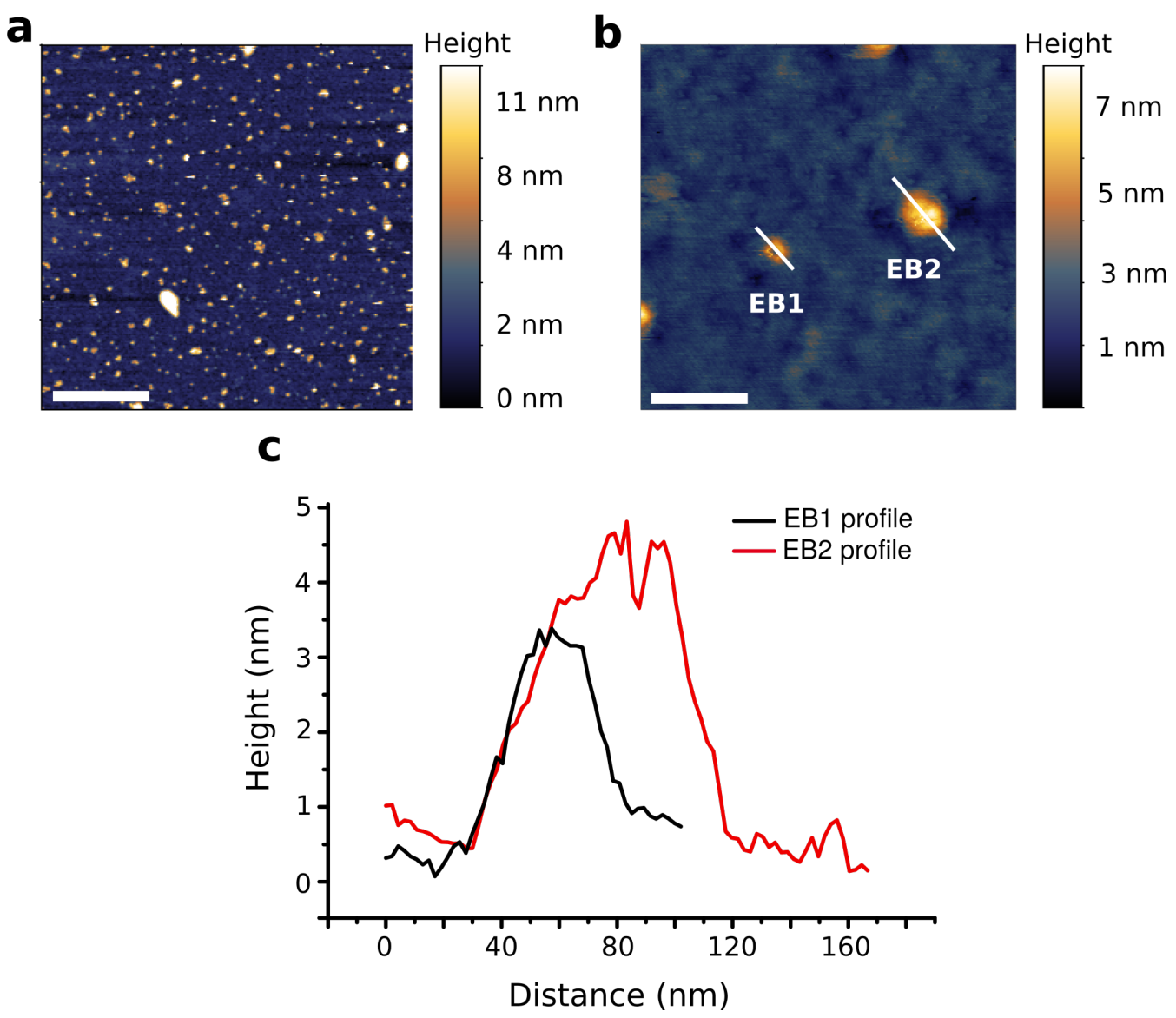

Figure 47: Topography of EB nanoparticles. (a) An overview of the surface morphology and particle size distribution of EB nanoparticles after 6h sonication. All images were obtained in tapping mode in air. The scale bar is $200 \mu \mathrm{m}$, (b) Multicolor image of the surface topography of two representative EB particles. Solid white lines mark the height profiles. The scale bar is $200 \mathrm{~nm}$, (c) Height profiles along the solid lines for EB1 and EB2 particles.

AFM measurements provided topographic mapping of EB nanoparticles and demonstrated their shape and height. As shown in Fig. 47k, the substrate roughness does not prevent us from resolving the z-height of EB particles. The dimensions of two EB particles depicted in Fig. 47b are approximately $40-80 \mathrm{~nm}$ length in $\mathrm{x}-\mathrm{y}$ dimension, and $3-4 \mathrm{~nm}$ height in z-direction. The size distribution for $\mathrm{N}=2865$ particles shows the following statistics:

(1) radius: $36 \pm 17 \mathrm{~nm}$, with values from 14.9 to $253.8 \mathrm{~nm}$

(2) height: $4.8 \pm 1.8$, with values from 1.5 to $11.2 \mathrm{~nm}$

Even after the exfoliation, 2D EB particles show the high aspect ratio that is suggested by its unit cell. Histograms of both height and radius distributions for EB nanosheets are shown in Fig. 48 . 

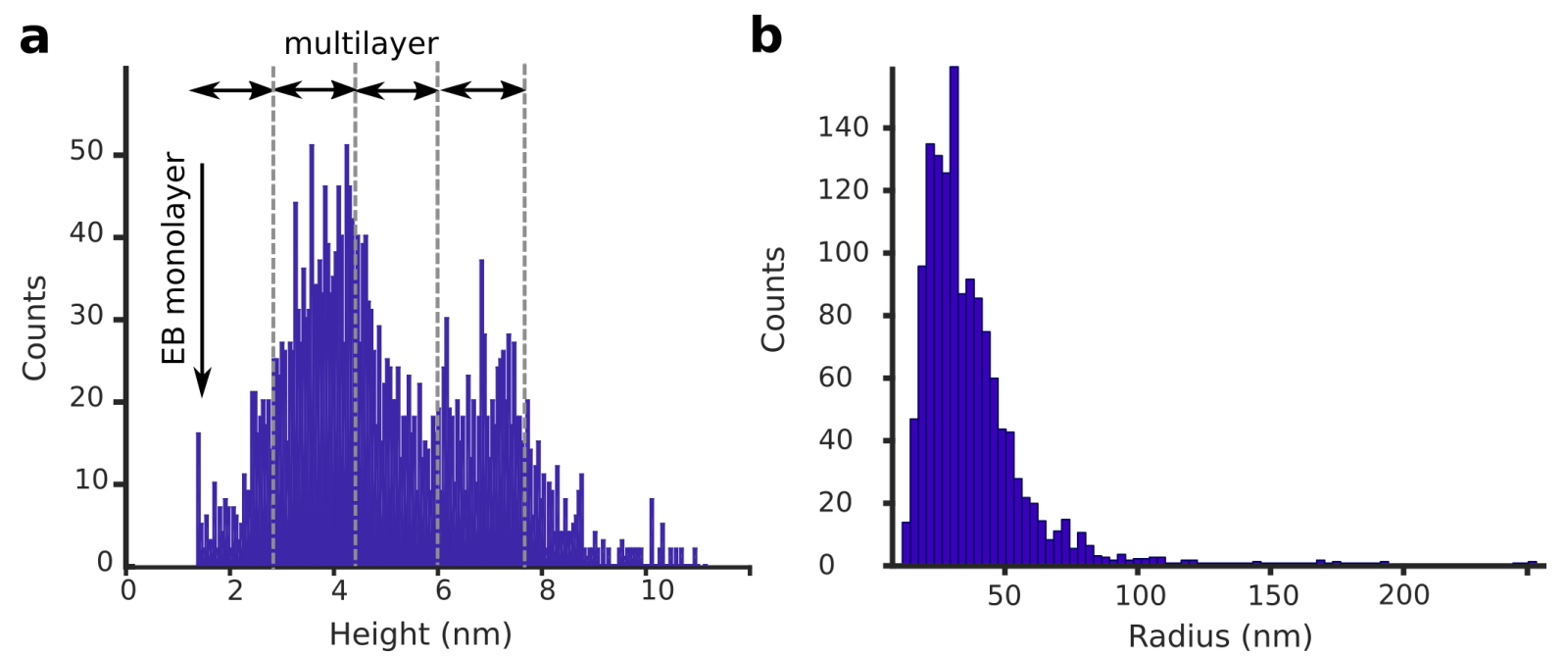

Figure 48: Dimensions of 2D EB nanosheets. Histograms of EB particle shape calculated from AFM topography images for (a) height and (b) radius.

The thickness of an individual EB monolayer was calculated to $0.9 \mathrm{~nm}[223]$. So far, all reported experimental values for monolayer EB nanosheet height were bigger than the theoretical thickness [273]. Possible reasons for the discrepancy are structural deformations caused by the exfoliation method, surface hydration of both EB nanoparticles and the silicon surfaces, and reduction of the coordination numbers of $\mathrm{Ca}^{2+}$ in monolayer structures in comparison to bulk samples. Latter can lead to a reorganization of the alkaline-earth metal ions in respect to the copper silicate network. Under these considerations, the smallest height of our 2D nanosheets $(1.5 \mathrm{~nm})$ is consistent with an individual EB single-layer thickness with aforementioned contributions. The data suggest that the majority of our EB nanosheets has the height of 3 monolayers. One should also consider that the collected height information is an average value and the exact height profile might vary individually, e.g. by featuring steps of different heights or multiple layer rafts.

\subsubsection{Photophysical properties of Egyptian Blue nanosheets}

After we established that the exfoliation process achieves small, relatively homogeneous particles, we need to investigate its photophysical properties. A sufficiently small nanosensor should still have enough fluorescence to enable optical investigation. Both spectroscopy and near-IR imaging show that nIR emission is preserved in exfoliated samples. To further investigate it, we will: (1) determine the fluorescent lifetime of the particles using a modulated excitation light beam, and (2) search for a possible correlation between the PL of a particle and its dimensions. 


\section{Fluorescence lifetimes}

Both quantum yield $\Phi$ and fluorescence lifetime $\tau$ are among the most essential fluorophore properties 208]. The time a fluorophore stays in the excited state is determined by the combination of the radiative rate constant $k_{\text {rad }}$ and the non-radiative rate constant $k_{n o n-r a d}$. Latter is usually a cumulative value, consisting of several non-radiative processes.

$$
\begin{gathered}
\Phi=\frac{k_{\text {rad }}}{k_{\text {rad }}+k_{\text {non-rad }}} \\
\tau=\frac{1}{k_{\text {rad }}+k_{\text {non-rad }}}
\end{gathered}
$$

While the radiative rate constant is almost unchangeable for a given molecule, the nonradiative rate constant is sensible to various deactivation pathways and therefore depends on the environment (e.g. presence of quenching molecules). There are two complementary pathways to measure the lifetime of a fluorophore: (1) the time domain and (2) the frequency domain. In the first technique (time domain), a short laser pulse excites fluorophore molecules. Then the decreasing number of excited fluorophores is measured against time. The fluorescence decay law postulates an exponential decay of excited molecules into the ground state and is based on the first-order kinetics:

$$
I(t)=I_{0} e^{\frac{-t}{\tau}}
$$

with $I(t)$ as the intensity after time $t, I_{0}$ as the initial intensity at $t=0, t$ as the time after excitation, and $\tau$ as the fluorescent lifetime. $\tau$ is calculated from the time-point when the fluorescence intensity $I(t)$ has decreased by a factor of 1/e. A monoexponential fit indicates a single fluorescent lifetime species. Multiexponential decay is caused by multiple emitters. The excitation pulse must be substantially shorter than the fluorescence lifetime of the analyte. Usually, the excitation happens on a nanosecond timescale to ensure an infinitesimally short $\delta$-pulse.

The second method (frequency domain) uses an excitation beam that is not pulsed but sinusoidally modulated. The emission of the sample repeats the excitation waveform, but is delayed in phase and modulated (Fig. 49a). For fluorophores with long lifetimes the phase shift increases, while the modulation ratio decreases. The fluorescence lifetime can be calculated from both the observed modulation factor (M) or the phase shift between excitation and emission waves $(\phi)$ [208]:

$$
\tau=\frac{1}{\omega} \tan (\phi)
$$




$$
\tau=\frac{1}{\omega} \sqrt{\frac{1}{M^{2}}-1}
$$

with $\omega$ as the angular excitation light frequency (radians per second) and $M$ as the modulation factor:

$$
M=\frac{b / B}{a / A}
$$

The modulation parameters are derived from the sinusoidally modulated curves of the excitation and emission waves: $L(t)=a+b \sin (\omega t)$ and $N(t)=A+B \sin (\omega t)$ [274]. For a system with only one emitter, both lifetimes obtained from the modulation and the phase shift calculation should be equal.
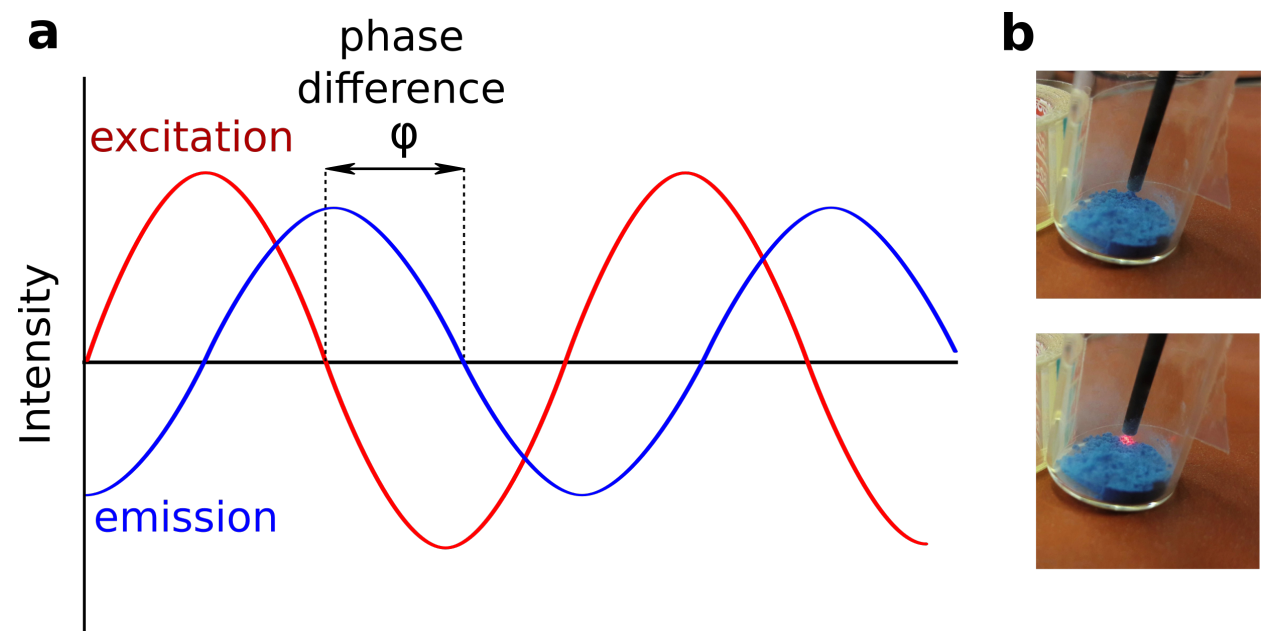

Figure 49: Fluorescence lifetime measurements in the frequency domain. (a) Schematics of the intensity-modulated light excitation (red) and the following emission curve (blue), with the phase shift $\phi$, (b) The tip of the fluorescence lifetime measurement device atop of an Egyptian Blue sample. For measurements the sensor tip was fully emerged into the sample.

For Egyptian Blue particles with reportedly long fluorescence lifetimes in the range of microseconds, the modulation approach was easier to perform. We used the FireSting O2 lifetime measurement device (PyroScience, Germany) with the excitation wavelength of $\lambda=$ $620 \mathrm{~nm}$ and the detection range of $700-920 \mathrm{~nm}(49 \mathrm{~b})$. The frequency of the excitation light was tested for the range of $100-5000 \mathrm{~Hz}$. 
Reported fluorescence lifetimes for Egyptian Blue lie between $107-130 \mu \mathrm{s}$ [215], [226]. The only calculated fluorescence lifetime for Han Blue was reported to be $75 \mu \mathrm{s}$ [275]. Our results are comparable with these findings and are presented in Table 5 .

Table 5: Fluorescence lifetimes of Egyptian Blue and Han Blue compounds calculated from the phase shift $\phi,(\mathrm{n}=3)$.

\begin{tabular}{ll} 
Sample & Fluorescence lifetime \\
\hline Egyptian Blue (powder) & $121.8 \pm 3.8 \mu \mathrm{s}$ \\
Egyptian Blue, after 6h sonication & $123.9 \pm 8.5 \mu \mathrm{s}$ \\
Han Blue (powder) & $52.1 \pm 4.7 \mu \mathrm{s}$
\end{tabular}

Fluorescence lifetimes values stay invariant within errorbars. From this finding one can conclude that both the exfoliation process and the reducing of dimensions have non or only a marginal effect on the optical properties of Egyptian Blue.

\section{Correlation between particle size and fluorescence}

In colloidal dispersions of nanomaterials, the exact size and shape of the nanoparticle often plays a crucial role for their optical spectrum. It is especially true in the case of such nanoparticles as gold, silver, and copper [276]. To investigate if a similar correlation can be observed in EB nanosheets, we analyzed the mean and single pixel intensity of the particles. Fig. 50 shows a series of nIR images of EB particles with the same preparation as for previous AFM measurements (6h tip sonication). The analysis was carried out with a matlab code, designed by Daniel Meyer and adjusted for the specifications of EB nanosheets.
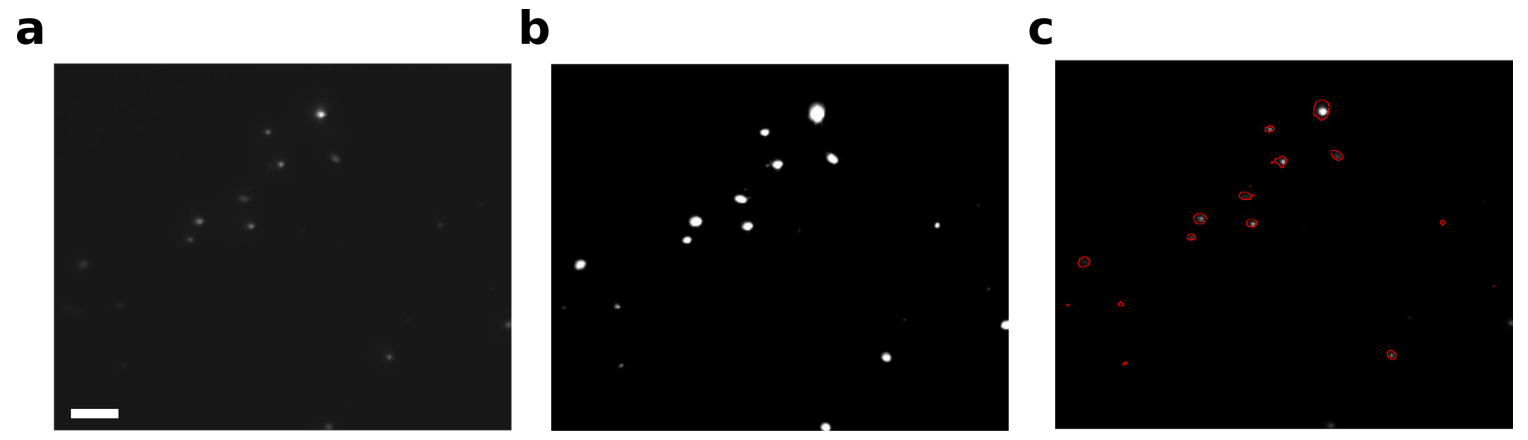

Figure 50: Particle analysis. (a) nIR image of EB nanosheets after $6 \mathrm{~h}$ sonication. The scale bar is $10 \mu \mathrm{m}$, (b) Intensity saturated version of (a) to guide the eye to the localization and size of nanosheets that are not apparent in the first image, (c) EB nanoparticles identified by the applied matlab program (designed by Daniel Meyer). 
Figure 50: illustrates positive identification of EB nanosheets. Compared to the bright field images that are often investigated in respect to the size and shape of captured objects (e.g. cell morphology and adherence), here one can observe one of the advantages of the nIR regime: the almost absent background fluorescence. Therefore, the images are analyzed without background correction. Instead the background evaluation is carried out by selecting 5 background spots. These 5 spots are then averaged, resulting in backval. In the next step, the particle intensity is defined by selecting 5 dimmest particles. Their mean intensity values define the lowest acceptable particle intensity level EBval. In the last step, the program sets a binary image with the following threshold:

$$
\begin{aligned}
& \text { fcbin }(\text { fcbin }<\text { backval }+(\text { EBval-backval }))=0 \\
& \text { fcbin }(\text { fcbin }>\text { backval }+(\text { EBval-backval }))=255
\end{aligned}
$$

Finally, every processed image delivers information of the mean intensity of the nanosheet, excluding those on the image edge. The distribution of mean intensity values is presented in Fig. 51 .
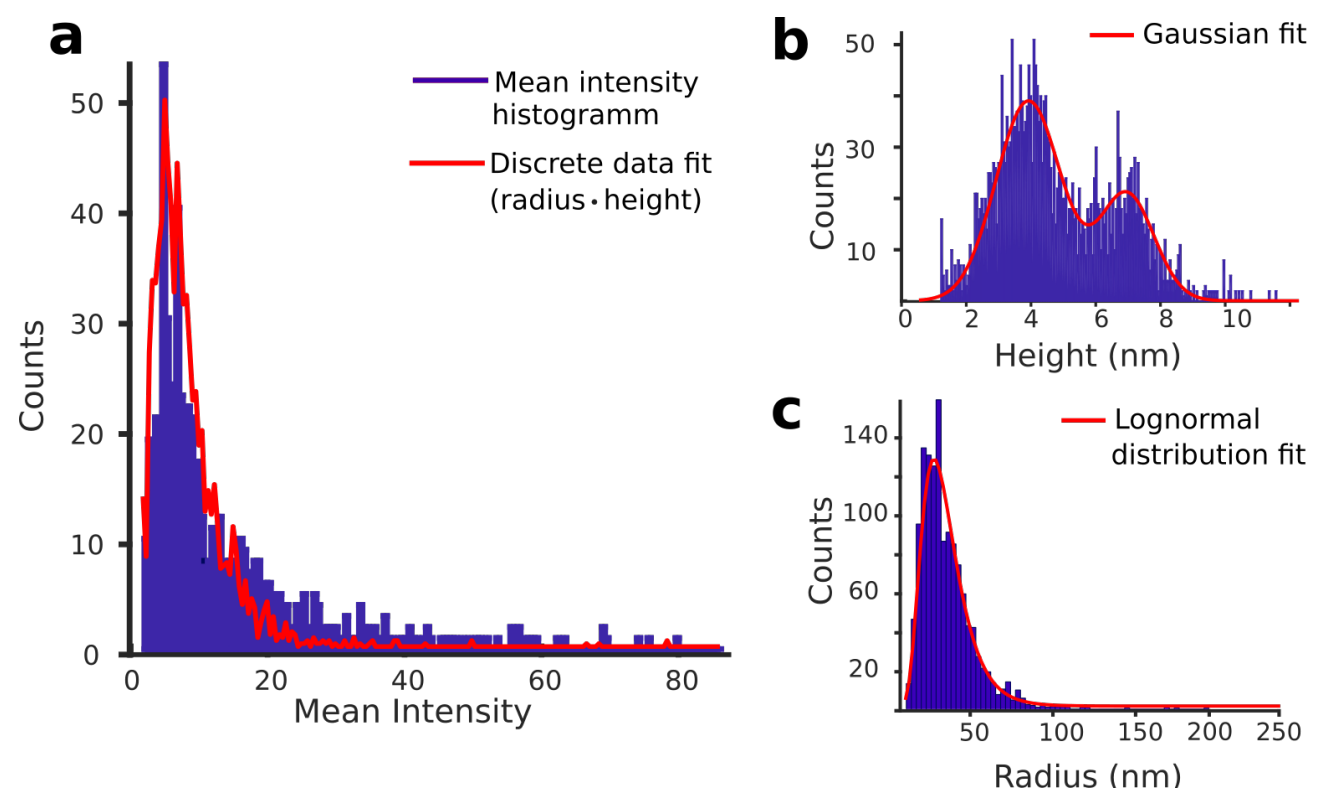

Figure 51: Mean intensity of EB nanosheets. (a) Mean intensity histogram for EB particles identified with the matlab code (blue bars). The fitting curve (red line) is based on discrete data obtained from AFM topography by multiplying the radius with the height of a particle. (b) Histogram of EB nanoparticles' height calculated from AFM topography images (blue bars). The fitting line is a Gaussian fit (red line), $R^{2}=0.9$ (c) Histogram of EB nanoparticles' radius calculated from AFM topography images (blue bars). The fitting line is a Lognormal distribution fit (red line). 
The histogram of mean intensities (Fig. 519) does not show any steps or distinct quantification of brightness levels. The mean intensity is relatively low and wide spread: 14 \pm 13 , spanning the values from 2 to 105. A slight dip in the mean intensity around 12 intensity counts might correspond to the two height maxima in the height distribution graph (Fig. 51b). However, the intensity minimum is not pronounced enough to allow clear interpretation. The fitting curve $F(z \cdot r)$ (Fig. 51 a, red line) that follows the distribution of mean intensities is based on the discrete values obtained from both height $z$ and radius $r$ data from AFM experiments. $F(z \cdot r)$ fitting curve represents a volume dependance of EB fluorescence.

To approximate the mean intensity of a defined EB nanoparticle, the following considerations can be employed: The height distribution can be fitted by the combination of two Gaussian curves (Fig. 51b):

$$
F(z)=39 \exp \left(-\frac{(z-3.5)}{1.4}\right)^{2}+21 \exp \left(-\frac{(z-6.5)}{1.2}\right)^{2}
$$

The radius distribution exhibits a skewness (asymmetry) of values and can be described by the Lognormal distribution (Fig. 51;):

$$
G(r)=\frac{1}{0.4 \sqrt{2 \pi} r} \exp \frac{-(\ln r-3.5)^{2}}{0.32}
$$

Following this calculation, the mean intensity of an EB nanoparticle of the given size can be estimated by:

$$
I(z, r)=F(z) \cdot G(r)
$$

It can be concluded that even a 2D nanosheet of EB exhibits nIR fluorescence and is therefore a promising building block for nIR-based biomedical imaging. 


\subsubsection{Egyptian Blue as ratiometric sensor for dopamine detection}

After we established that 2D nanoparticles of Egyptian Blue exhibit stable fluorescence in the nIR range, we can incorporate them in dopamine sensors. The idea is to observe the emission maximum of Egyptian Blue $\left(\lambda_{E B}=930 \mathrm{~nm}\right)$ alongside with the emission maximum of ssDNA/SWCNTs $\left(\lambda_{D N A / C N T}=990 \mathrm{~nm}\right)$. In a ratiometric sensor design the output is given by the ratio of two emission signals. To achieve this, one of the fluorophores has to respond to the addition of analyte and the other fluorophore has to stay invariant (reference signal). This technique is generally very robust because it is not affected by variations of absolute intensity values that can vary depending on the background illumination, light source fluctuations, or concentration changes. A common drawback of ratiometric sensors is the need for changing of excitation/emission wavelengths at suitable rates. Since both Egyptian Blue and SWCNTs can be excited at the same wavelength and collected within similar detector range this can be avoided.

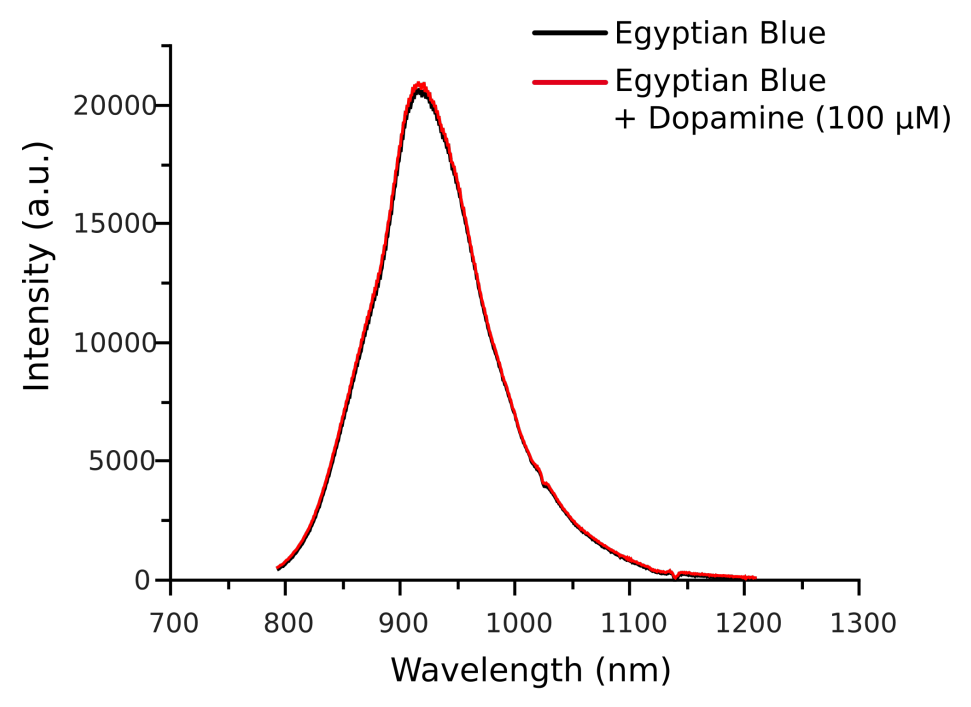

Figure 52: Egyptian Blue does not respond to dopamine addition. Emission spectrum of EB nanoparticles immobilized on a surface before (black curve) and after (red curve) the addition of $100 \mu \mathrm{M}$ dopamine $\left(\lambda_{e x}=560 \mathrm{~nm}\right)$.

The first step is to ensure that EB does not respond to dopamine. After addition of $100 \mu \mathrm{M}$ dopamine to EB immobilized on a glass surface, there were observed neither any changes in the intensity nor a shift in the emission wavelength (Fig. 52a). Furthermore, the library of possible analytes was extended to include: neurotransmitters (dopamine, $\gamma$-aminobutyric acid), amino acids (cysteine, phenylalanine, etc.), and other possible interfering substances (such as EDTA, as the chelating agent for copper ions). Emission intensities and maximum wavelengths before and after addition of analytes lied within the error-range (these studies were conducted by Master student Alexander Spreinat during a one month internship). 
EB samples also did not respond to temperature- or pH-changes. It indicates that the silicate tetrahedrals around copper efficiently shield it from environmental influences. This characteristic is suitable for biological application as a stable fluorophore and in its capacity as a reference signal.
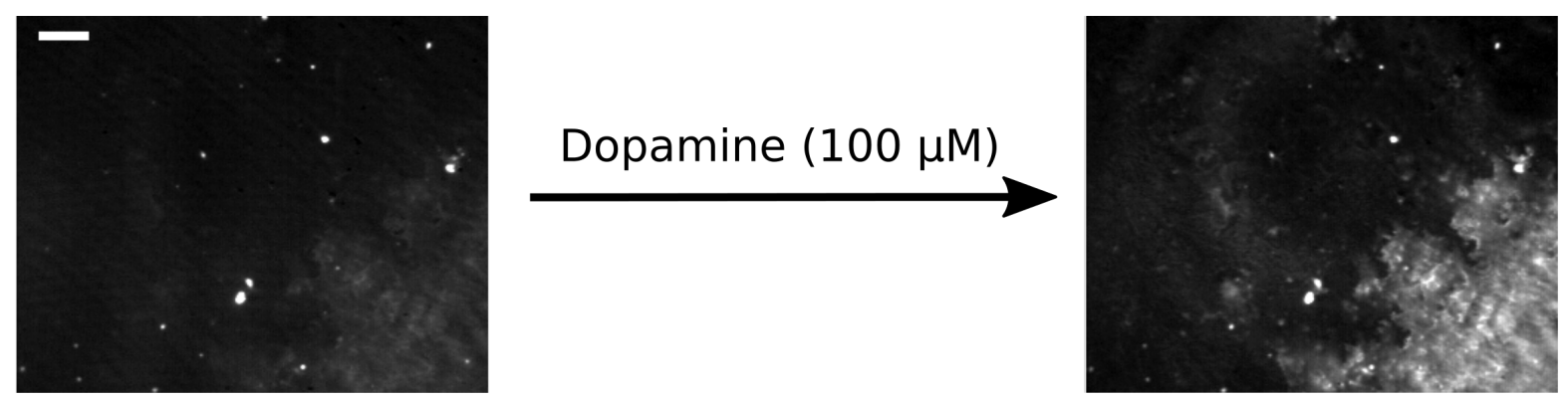

Figure 53: nIR fluorescence images of EB and $(\mathrm{GT})_{15} /$ SWCNTs immobilized on a glass surface. After addition of dopamine $(100 \mu \mathrm{M})$, the spots of Egyptian Blue remain the same in their intensity, while the patch of $(\mathrm{GT})_{15} / \mathrm{SWCNTs}$ (lower right corner) gets significantly brighter. The scale bar is $50 \mu \mathrm{m}$.

The ratiometric studies were performed in solution, after EB particles sank to the bottom of the well. Possible variations in the intensity signal might come from the insufficient adherence of particles onto the glass surface of the well. When the well was stirred or physically moved from the holder, smaller EB particles could shift in place. However, with cautious addition of analytes there were observed no disturbances. The nIR image shown in Figure 53 illustrates such an example. Bright spots in the first image are mostly EB particles, with a dim outline of immobilized $(\mathrm{GT})_{15} /$ SWCNTs in the right lower corner of the image. After addition of dopamine $(100 \mu \mathrm{M})$, the spots of EB stay the same in their intensity, while the patch of $(\mathrm{GT})_{15} /$ SWCNTs gets significantly brighter.

The same phenomenon can be observed in Fig. $54 \mathrm{~b}$. It shows the emission spectrum of EB and $(\mathrm{GT})_{15} /$ SWCNTs before (black curve) and after (red curve) the addition of 100 $\mu \mathrm{M}$ dopamine. The calibration curve of ratiometric response of EB and $(\mathrm{GT})_{15} / \mathrm{SWCNTs}$ after addition of dopamine shows that the linear regime of the sensor lies in approx. 10 $\mathrm{nM}-10 \mu \mathrm{M}$. At higher concentrations $(>10 \mu \mathrm{M})$, the sensor response saturated and the variations of response increased. That effect might be caused by dopamine polymerization at high concentrations. 

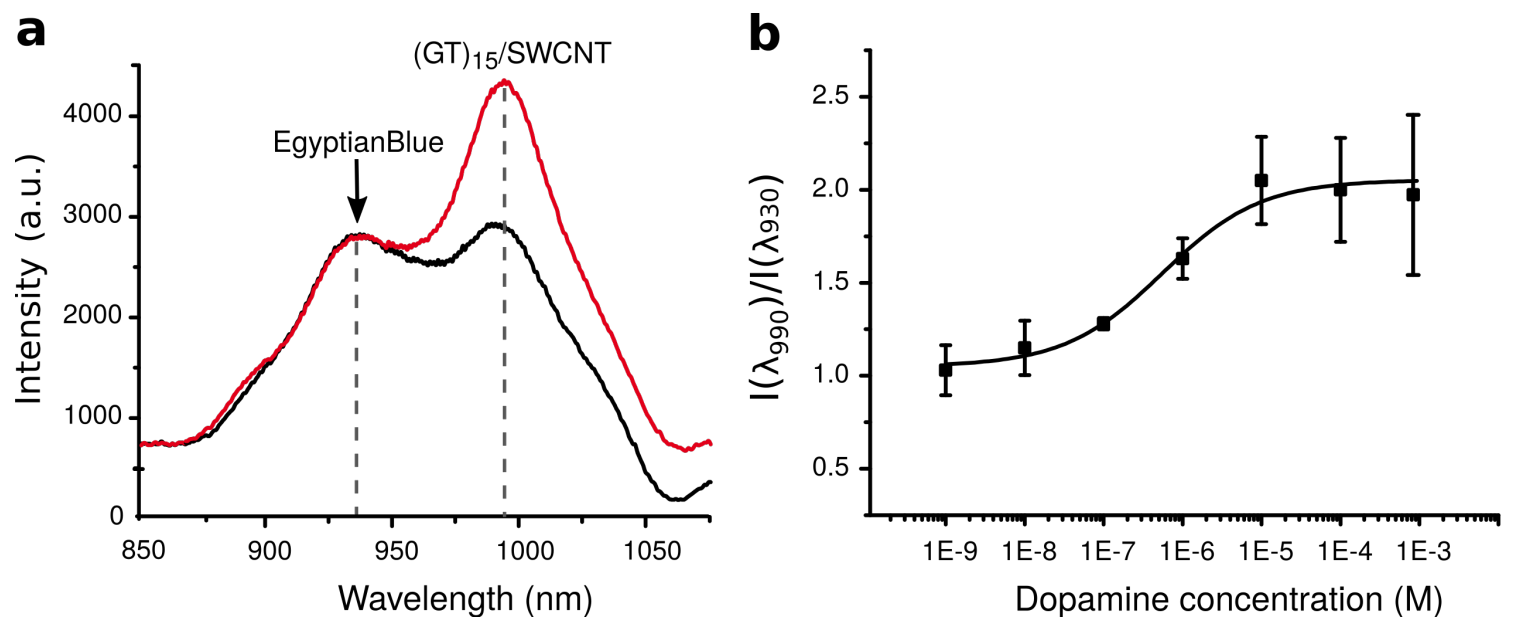

Figure 54: Egyptian Blue as a reference signal for (ratiometric) dopamine detection.

(a) Emission spectrum of Egyptian Blue and $(\mathrm{GT})_{15} / \mathrm{SWCNTs}$ in solution before (black curve) and after (red curve) addition of $100 \mu \mathrm{M}$ dopamine $\left(\lambda_{E B}=930 \mathrm{~nm}\right.$, $\lambda_{D N A / C N T}=990 \mathrm{~nm}, \lambda_{e x}=560 \mathrm{~nm}$ ), (b) Calibration curve of ratiometric response of Egyptian Blue and $(\mathrm{GT})_{15} /$ SWCNTs in solution after addition of dopamine (error bars are standard deviations, $\mathrm{n}=3)$. The black line denotes the logistic fit $\left(R^{2}=\right.$ $0.97)$.

To characterize the calibration curve, a four parameter logistic fit was employed (kinetic adsorption model, $\left.R^{2}=0.97\right)$ :

$$
y=A_{2}+\frac{\left(A_{1}-A_{2}\right)}{\left(1+\left(\frac{x}{x_{0}}\right)^{p}\right)}
$$

with the following parameters:

$A_{1}$ (minimal ratiometric response $)=1.05 \pm 0.06$

$A_{2}$ (maximal ratiometric response) $=2.06 \pm 0.09$

$x_{0}$ (the point of inflection or $\left.K_{d}\right)=574 \pm 233 \mathrm{nM}$

$p$ (steepness of the curve at point $\left.x_{0}\right)=0.69 \pm 0.14$

$p<1$ indicates negative cooperativity. That means that the first binding of dopamine to EB-(GT) ${ }_{15} /$ SWCNTs sensors changes the binding affinity for the second dopamine molecule, making it less likely. These sensor kinetics are comparable to the normal $(\mathrm{GT})_{15} / \mathrm{SWCNTs}$ sensor kinetics without additional ratiometrical particles [54]. Thus, one can conclude that the binding kinetics of dopamine to $(\mathrm{GT})_{15}$ /SWCNTs sensors are nor disturbed by addition of Egyptian Blue. 


\subsubsection{Conclusion}

In this section, we investigated the novel nIR fluorophore: Egyptian Blue (EB). First, we analyzed the morphology of exfoliated EB particles via atomic force microscopy. The exfoliation process produced 2D nanosheets: elongated and flat, with a radius of approximately $36 \mathrm{~nm}$ and a thickness between 4 and $5 \mathrm{~nm}$. This height corresponds to 3 or 4 multilayers of EB unit cells. Monolayer EB nanosheets were also produced, albeit not as the major species. Then we showed that both bulk and nanosheet structures are strong nIR emitters. No changes in fluorescent lifetimes from bulk to single molecules could be detected $(\tau \approx$ $124 \mu \mathrm{s})$, which suggests that the dimensionality of the particles does not correlate with their fluorescence quantum yield. The distribution of mean intensity of $2 \mathrm{D}$ EB nanosheets can be described as the product of height and radius of the particles. With this we could show that the fluorescence properties of EB are preserved in nanosheets and correlate with the nanosheets volume. Stable photoluminescence of EB nanosheets can be used in nIR bioimaging. Therefore, we implemented EB as a nIR reference signal for ratiometric dopamine detection. These insights not only allow for a better understanding of the photochemical properties of an ancient pigment, but also expand the promising class of nIR fluorophores to a new $2 \mathrm{D}$ nanomaterial. 


\subsubsection{Outlook for new nIR fluorophores}

The investigation of EB nanosheets has just started and there are several pathways that are worth exploring in future projects:

- Further size reduction with the goal of homogenous monolayer structures, preferably down to a single unit cell. The size reduction might require prolonged sonication or heat exposure times (up to two weeks according to [272]). After the exfoliation, a subsequent isolation of smallest particles should follow (either with suitable filters or by employing a size exclusion column). This would be the first step for all following applications.

- Water solubility of Egyptian Blue particles is crucial for biological applications, yet it still remains a challenge. Functionalization of EB monolayers (e.g. with amino residues via silanization [275]) could not only render them soluble but also provide a basis for attachment of an antibody or a peptide recognition unit.

- EB is a nanomaterial with very stable fluorescence. This is an advantage for application as a reference signal or a staining fluorophore. So far, EB showed no sensitivity for any analyte, which could make sensor development rather difficult. However, when an analyte that affects its fluorescence can be found, this will result in a very selective and robust sensor.

- Fluorescence of EB is described by the ligand splitting field theory. The field energy depends on the distance between the ligands and the metal ion. It was shown that lattice expansion due to bigger earth-alkali metals ions correlates with the emission red-shift [221], 224]. Therefore, an application as an optical force sensor or a mechanosensor should be possible. A first step for this application would be to achieve a calibration curve of wavelength shifts vs. applied pressures.

- Copper nanoparticles are known for their antimicrobial activity 277]. However, rapid copper oxidation hinders any progress in this area. Instead, stable copper-based 2D nanosheets could present a suitable alternative. For this application EB nanosheets should also be tested as possible antifungal/antibacterial agents.

- Finally, there is also the idea of using EB nanosheets in a 'security' device, e.g. by injecting money or official documents with invisible security ink based on EB that would only show in nIR. 


\section{Conclusion and outlook}

The particular conclusions of each of the three parts of the thesis were already discussed in detail after their respective chapter. Therefore, in the final part I would like to briefly summarize the individual achievements and tie them into the global picture of bionanosensor development.

The aim of this study was to test strategies that facilitate nIR sensor development for detection of small molecules, e.g. neurotransmitters. As basic sensor units we used near-infrared (nIR) fluorophores, such as single-walled carbon nanotubes (SWCNTs) and Egyptian Blue (EB). First, we focused on SWCNTs as sensor building blocks. SWCNTs are most suitable for biological applications due to their emission wavelength of $850-1700 \mathrm{~nm}$. The organic phase (polymer) around SWCNTs governs their selectivity. For rational sensor design a better understanding of the underlying sensor mechanism is essential. However, the exact mechanism of the interaction between analyte and polymer/SWCNTs is not yet understood.

To answer this question, we investigated several of the mechanisms that were proposed to be responsible for the fluorescence change. The change of SWCNT fluorescence can be induced by a variety of causes: redox chemistry, availability of free surface area around the SWCNT, conformational changes of the organic phase, etc. In the first section, we investigated how small redox-active molecules affect the fluorescence change in SWCNT-based sensors. Here, we put emphasis on systematically changing the organic phase around the SWCNT. Interestingly, the data suggest that there is a correlation between the charge of the polymer and the sensor response. From the proposed mechanisms we have ruled out a direct electron transfer between the analyte and the SWCNT, and verified that the fluorescence increase does not come from an analyte-induced decrease of reactive oxygen species. Furthermore, we showed that there is no correlation between the free surface space of SWCNT and the sensor response, thus eliminating a sensing mechanism based on pure adsorption of a polymer onto the SWCNT. The results provide evidence for a sensing mechanism based on the conformational change of a polymer, which is also supported by MD simulations.

Based on the finding that the polymer plays a central role in the interaction between analytes and polymer/SWCNTs, we advanced the polymer synthesis strategies. In the next chapter we introduced a novel hybrid polymer that combines high sensitivity of ssDNA/SWCNT sensors with the selectivity of specific peptide recognition units. This modular approach allows to modulate the binding affinity of the recognition unit via small changes of one of the sensor components. As proof of principle this strategy was tested for a well-established integrin recognition unit: Arg-Gly-Asp (RGD). In this chapter, we quantified how different ssDNA-peptide/SWCNT hybrids affect the binding of RGD-motif to the $\alpha_{I I b} \beta_{3}$ integrin via an enzyme-linked immunosorbent binding assay (ELISA). The data showed that small 
changes in one of the sensor units can change the IC50 values from $20 \mathrm{nM}$ to $309 \mathrm{nM}$. These results suggest that small changes in all three parameters, namely ssDNA sequence, length and geometry, modulate the affinity of ssDNA-RGD/SWCNTs binding to $\alpha_{I I b} \beta_{3}$ integrin. Here, we also introduced a new method of determining the stoichiometry of recognitionmotifs in SWCNTs-based sensors. Finally, we labeled $\alpha_{I I b} \beta_{3}$ integrin on blood platelets with the most promising ssDNA-RGD/SWCNTs complexes. After we established that this strategy works, the technique can be adapted for other molecules, e.g. for the neurotransmitter GABA. For those sensors, one could use the peptide sequence responsible for GABA recognition, bind it to the ssDNA sequence and adjust the surrounding of the peptide to mimic the geometry of the binding protein. High-throughput screening of various combinations might help to identify the most promising candidates.

One of the goals of bionanosensor research is to implement the new sensors in clinical applications. Aside from neurotransmitter detection, targeting melanoma cells or delivering drugs into a diseased area might be an important application. Other possibilities include sensors to detect multidrug-resistant bacteria cultures in clinics and public facilities. Therefore, in the last section we added Egyptian Blue, a bright nIR emitter, into the small library of existing nIR fluorophores. First, we analyzed the morphology of exfoliated Egyptian Blue nanosheets via atomic force microscopy. Then, we demonstrated that both bulk and quasi 2D nanosheet structures are strong nIR emitters. We investigated their photophysical properties and showed that the dimensionality of the nanosheets does not affect the fluorescence quantum yield. The fluorescent lifetimes of both materials are in the range of microseconds. The distribution of mean intensity of 2D Egyptian Blue nanosheets could be described by the product of height and radius of the particles, and therefore correlates with the nanosheet volume. Therefore, even a single Egyptian Blue unit cell should exhibit nIR fluorescence. Finally, we demonstrated a successful application of Egyptian Blue nanosheets in a ratiometric sensor approach for dopamine detection. These insights expand the promising class of nIR fluorophores to a new two-dimensional nanomaterial.

In summary this study has demonstrated:

1. that SWCNT-based sensors change their fluorescence due to conformational changes of the polymer around the SWCNT

2. a modular approach to tune the organic phase of SWCNTs to increase the selectivity, and

3. the use of Egyptian Blue as a novel nIR fluorophore for various detection and nIR staining techniques. 


\section{Appendix}

\section{Absorption and emission spectra of all ssDNA-peptide/SWCNT conjugates for RGD reconition motif modulation}
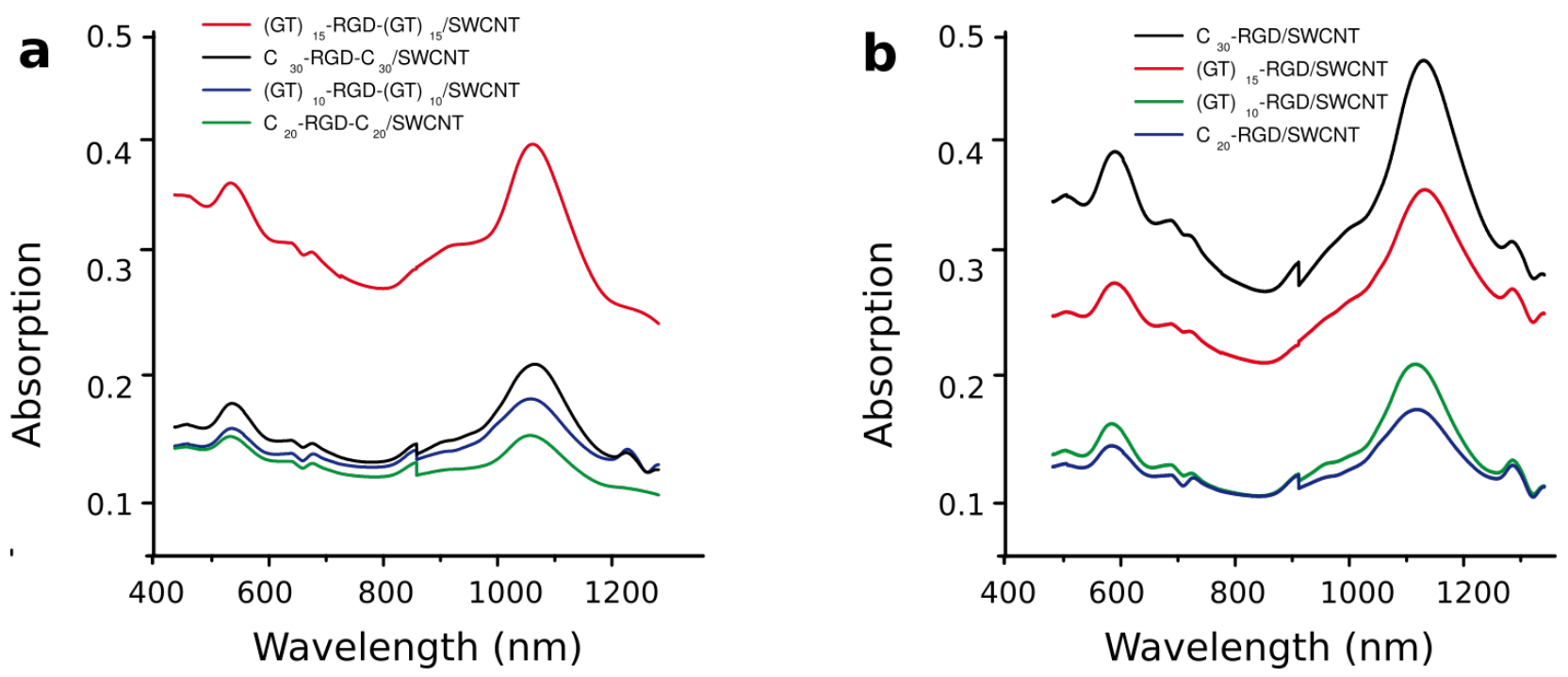

Figure 55: Absorption spectra of ssDNA-peptide/SWCNT hybrids.
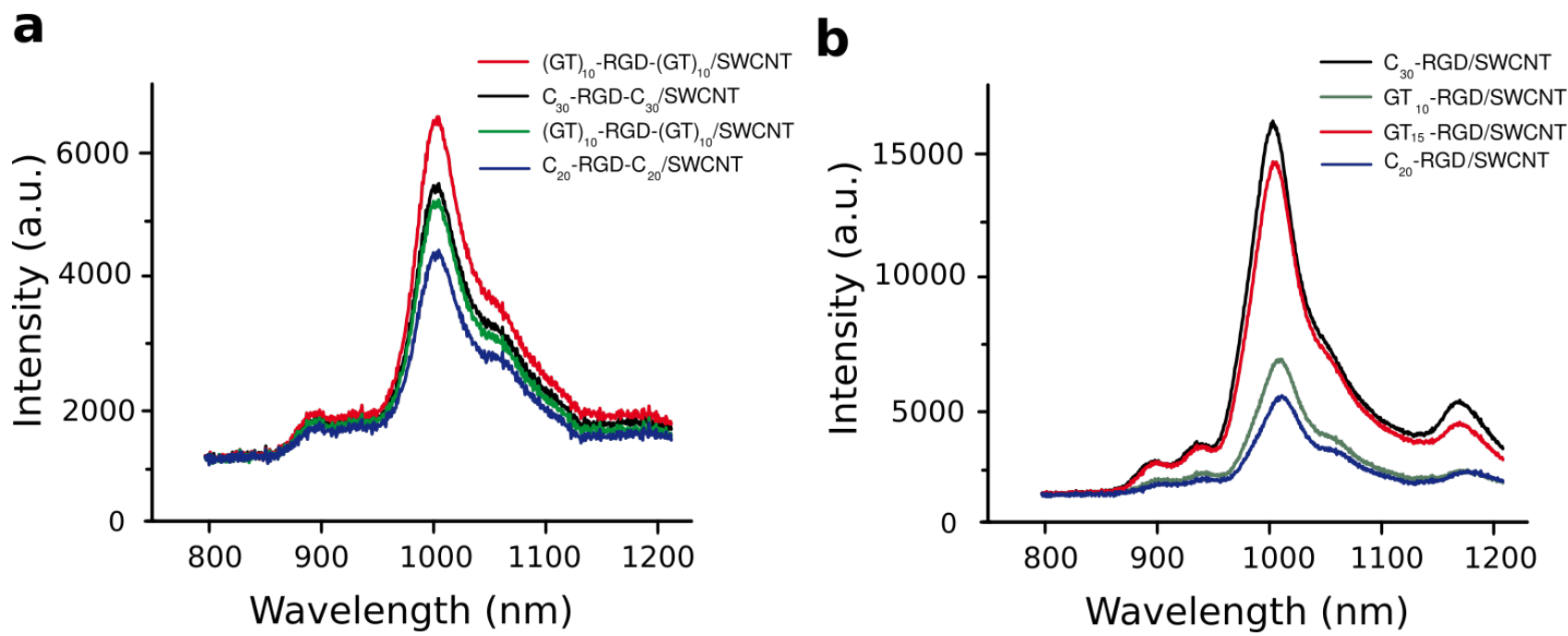

Figure 56: Emission spectra of ssDNA-peptide/SWCNT hybrids. 


\section{Adhesion of MDCKII cells seeded on various ssDNA-RGD/SWCNT hybrids}
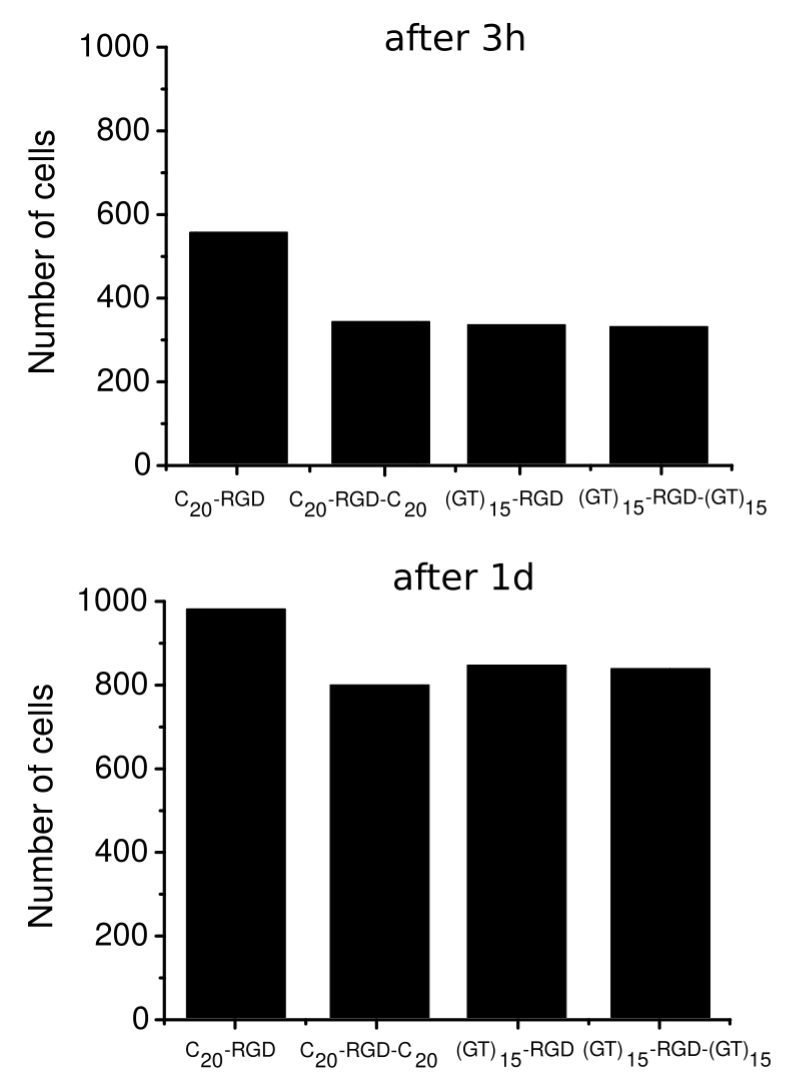

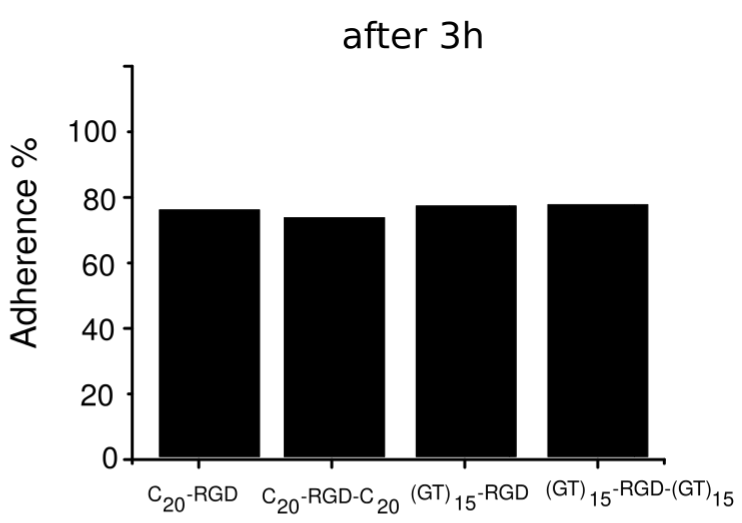

after $1 d$

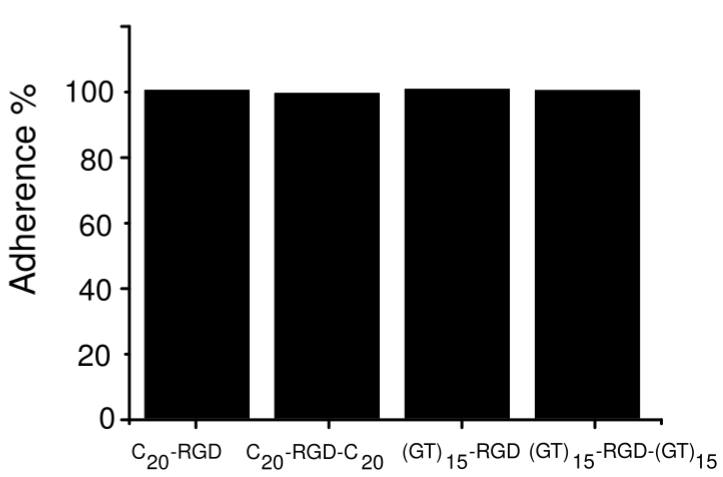

Figure 57: Adhesion of MDCKII cells seeded on various ssDNA-RGD/SWCNT hybrids. Development of cells on four glass coating containing RGD-motif: $\mathrm{C}_{20^{-}}$ RGD/SWCNT, $\mathrm{C}_{20}$-RGD-C 20 /SWCNT, (GT) 15 -RGD/SWCNT, and (GT) 15 -RGD$(\mathrm{GT})_{15} /$ SWCNTs. Graphs show the percentage of adherent cells and the total number of cells 3 hours and 1 day after seeding on substrates (the values are normalized to the values on glass surface). 


\section{Egyptian Blue exfoliation methods}

Table 6: Various exfoliation techniques for size reduction of Egyptian Blue compounds, collected by nIR imaging and analyzed by ImageJ particle recognition plugin.

Exfoliation method

$2 \mathrm{~h}$ in water at $75^{\circ} \mathrm{C}+$ stirring

$2 \mathrm{~h}$ of tip sonication

double adhesive tape
Mean particle area Observation overall biggest particles small, mostly homogeneous particles smallest particles but inhomogeneous

Table 7: Size of Egyptian Blue particles after various sonication times, values are derived from DLS measurement.

\begin{tabular}{ll} 
Sonication time $(\mathbf{m i n})$ & Diameter of particles $(\mathbf{n m})$ \\
\hline $0($ EB powder $)$ & 712 \\
30 & 735 \\
60 & 454 \\
120 & 365 \\
120, additional filter of $0.45 \mu \mathrm{m}$ & 132 \\
120, additional filter of $0.2 \mu \mathrm{m}$ & 108
\end{tabular}




\section{References}

[1] S. Herculano-Houzel. The human brain in numbers: a linearly scaled-up primate brain. Front Hum Neurosci, 3(31):1-11, 2009.

[2] B. Haider. The war of the soups and the sparks: The discovery of neurotransmitters and the dispute over how nerves communicate. The Yale Journal of Biology and Medicine, 80(3):138-139, 2007.

[3] J. N. Stuart, A. B. Hummon, and J. V. Sweedler. The chemistry of thought neurotransmitters in the brain. Anal Chem, 2004.

[4] T. C. Suedhof. The synaptic vesicle cycle. Annu Rev Neurosci, 27:509-47, 2004.

[5] S. E. Hyman. Neurotransmitters. Curr Biol, 15(5):154-158, 2005.

[6] E. Polo and S. Kruss. Nanosensors for neurotransmitters. Anal Bioanal Chem, 408:2727-2741, 2016.

[7] S. J. Cragg and M. E. Rice. Dancing past the dat at a da synapse. Trends Neuroscience, 27(5):270-277, 2004.

[8] S. Olah, M. Fule, G. Komlosi, C. Varga, R. Baldi, P. Barzo, and G. Tamas. Regulation of cortical microcircuits by unitary gabamediated volume transmission. Nature, 461(7268):1278-1281, 2009.

[9] T. S. Hnasko and R. H. Edwards. Neurotransmitter corelease: mechanism and physiological role. Annu Rev Physiol, 74:225-243, 2012.

[10] D. Dulcis, P. Jamshidi, S. Leutgeb, and N. C. Spitzer. Neurotransmitter switching in the adult brain regulates behavior. Science, 340(6131):449-453, 2013.

[11] A. R. Damasio, M. J. Farah, M. F. Huerta, and J. H. Schwartz. Encyclopedia of the Human Brain. Elsevier Science Ltd., 2002.

[12] Maeve Fitzgerald and Estomih Mtui. Clinical Neuroanatomy and Neuroscience. Saunders Elsevier, 2012.

[13] B. Rubi and P. Maechler. Minireview: new roles for peripheral dopamine on metabolic control and tumor growth. Endocrinology, 151(12):5570-5581, 2010.

[14] J. Bergquist, A. Tarkowski, R. Ekman, and A. Ewing. Discovery of endogenous catecholamines in lymphocytes and evidence for catecholamine regulation of lymphocyte function via an autocrine loop. Proc Natl Acad Sci USA, 91(26):12912-12916, 1994. 
[15] R. A. Wise. Dopamine, learning and motivation. Nature Reviews Neuroscience, 5:483494, 2004.

[16] G. M. Petzinger, D.P. Holschneider, B. E. Fisher, S. McEwen, N. Kintz, M. Halliday, W. Toy, J. W. Walsh, J. Beeler, and M. W. Jakowec. The effects of exercise on dopamine neurotransmission in parkinson's disease: Targeting neuroplasticity to modulate basal ganglia circuitry. Brain Plast., 1(1):29-39, 2015.

[17] J.-M. Beaulieu, R. R. Gainetdinov, and R. Sibley. The physiology, signaling, and pharmacology of dopamine receptors. Pharmacological Reviews March, 63:182-217, 2011.

[18] R. H. Westerink and A. G. Ewing. The pc12 cell as model for neurosecretion. Acta Physiol (Oxf), 192(2):273-285, 2008.

[19] Claire Ribrault, Ken Sekimoto, and Antoine Triller. From the stochasticity of molecular processes to the variability of synaptic transmission. Nature Reviews Neuroscience, 12:375-387, 2011.

[20] J. R. Geiger and P. Jonas. Dynamic control of presynaptic ca2+ inflow by fastinactivating $\mathrm{k}+$ channels in hippocampal mossy fiber boutons. Neuron, 28:927-939, 2000 .

[21] D. Choquet and A. Triller. The role of receptor diffusion in the organization of the postsynaptic membrane. Nature Reviews Neuroscience, 4:251-265, 2003.

[22] Daniel Meyer, Annika Hagemann, and Sebastian Kruss. Kinetic requirements for spatiotemporal chemical imaging with fluorescent nanosensors. ACS Nano, 11(4):40174027, 2017.

[23] Elliot S. Valenstein. The discovery of chemical neurotransmitters. Brain and Cognition, 49:73-95, 2002.

[24] T. Zetterström, T. Sharp, C. A. Marsden, and U. Ungersted. In vivo measurement of dopamine and its metabolites by intracerebral dialysis: changes after d-amphetamine. Journal of Neurochemistry, 41(6):1769-73, 19983.

[25] P. T. Kissinger, C. S. Bruntlett, G. C. Davis, L. Felice, R. M. Riggin, and R. E. Shoup. Recent developments in the clinical assessment of the metabolism of aromatics by high-performance, reversed-phase chromatography with amperometric detection. Clin Chem, 23(8):1449-55, 1977.

[26] K. F. Faull. Application of combined gas chromatography/mass spectrometry to neurotransmitter research. Life Sci., 41(7):889-92., 1987. 
[27] J. Kehr. Determination of glutamate and aspartate in microdialysis samples by reversed-phase column liquid chromatography with fluorescence and electrochemical detection. J Chromatogr B Biomed Sci Appl, 4(708):27-38, 1998.

[28] P. T. Kissinger, J. B. Hart, and R. N. Adams. Voltammetry in brain tissue: a new neurophysiological measurement,. Brain Res., 55:209-13, 1973.

[29] Fiona E. Harrison and James M. May. Vitamin c function in the brain: Vital role of the ascorbate transporter (svct2). Free Radic Biol Med., 46(6):719-730, 2009.

[30] R. M. Wightman. Monitoring molecules: insights and progress. ACS Chem Neurosci, $6(1): 5-7,2015$.

[31] E. S. Bucher R. M. Wightman. Electrochemical analysis of neurotransmitters. Annu Rev Anal Chem, 8:239-61, 2015.

[32] Thomas L. Colliver and Andrew G. Ewing. Electrochemical Detection of Neurotransmitters. John Wiley \& Sons, Ltd, 2006.

[33] Roland G. W. Staal, Stephen Rayport, and David Sulzer. Electrochemical Methods for Neuroscience. Amperometric Detection of Dopamine Exocytosis from Synaptic Terminals. CRC Press/Taylor \& Francis, 2007.

[34] R. M. Wightman. Temporally resolved catecholamine spikes correspond to single vesicle release from individual chromaffin cells. Proc. Nail. Acad. Sci. USA, 88:1075410758, 1991.

[35] E. V. Mosharov and D. Sulzer. Analysis of exocytotic events recorded by amperometry. Nature Methods, 2(9):651-8, 2005.

[36] I. Hafez, K. Kisler, K. Berberian, G. Dernick, V. Valero, M. G. Yong, H. G. Craighead, and M. Lindau M. Electrochemical imaging of fusion pore openings by electrochemical detector arrays. Proc Natl Acad Sci USA, 102(39):13879-13884, 2005.

[37] J. Wang, R. Trouillon, Y. Lin, M. I. Svensson, and A. G. Ewing. Individually addressable thin-film ultramicroelectrode array for spatial measurements of single vesicle release. Anal Chem, 85(11):5600-5608, 2013.

[38] Jun Wang and Andrew G. Ewing. Simultaneous study of subcellular exocytosis with individually addressable multiple microelectrodes. Analyst, 139:3290-3295, 2014.

[39] Brian M. Kile, Paul L. Walsh, Zoé A. McElligott, Elizabeth S. Bucher, Thomas S. Guillot, Ali Salahpour, Marc G. Caron, and R. Mark Wightman. Optimizing the temporal resolution of fast-scan cyclic voltammetry. ACS Chemical Neuroscience, 3(4):285-292, 2012. PMID: 22708011. 
[40] R. Mark Wightman. Probing cellular chemistry in biological systems with microelectrodes. Science, 311(5767):1570-1574, 2006.

[41] D. L. Robinson, B. J. Venton, M. L. Heien, and R. M. Wightman. Detecting subsecond dopamine release with fast-scan cyclic voltammetry in vivo. Clin Chem, 49(10):1763$73,2003$.

[42] Yu-Tao Li, Shu-Hui Zhang, Li Wang, Rong-Rong Xiao, Wei Liu, Xin-Wei Zhang, Zhuan Zhou, Christian Amatore, and Wei-Hua Huang. Nanoelectrode for amperometric monitoring of individual vesicular exocytosis inside single synapses. Angewandte Chemie International Edition, 53(46):12456-12460, 2014.

[43] Xianchan Li, Soodabeh Majdi, Johan Dunevall, Hoda Fathali, and Andrew G. Ewing. Quantitative measurement of transmitters in individual vesicles in the cytoplasm of single cells with nanotip electrodes. Angewandte Chemie International Edition, 54(41):11978-11982, 2015.

[44] Bo Zhang, Michael L. A. V. Heien, Michael F. Santillo, Lisa Mellander, and Andrew G. Ewing. Temporal resolution in electrochemical imaging on single pc12 cells using amperometry and voltammetry at microelectrode arrays. Anal. Chem, 83(2):571-577, 2011.

[45] R. Freeman, L. Bahshi, T. Finder, R. Gill, and I. Willner. Competitive analysis of saccharides or dopamine by boronic acid-functionalized cdse-zns quantum dots. Chem Commun (Camb), 7:764-766, 2009.

[46] Rumei Cheng, Congcong Ge, Lei Qi, Zhengyun Zhang, Jinlei Ma, Huiying Huang, Tonghe Pan, Quanbin Dai, and Liming Dai. Label-free graphene oxide förster resonance energy transfer sensors for selective detection of dopamine in human serums and cells. The Journal of Physical Chemistry C, 0(0):null, 0.

[47] J. L. Chen, X. P. Yan, K. Meng, and S. F. Wang. Graphene oxide-based photoinduced charge transfer label-free near-infrared fluorescent biosensor for dopamine. Anal Chem, 82(22):8787-8793, 2011.

[48] R. Liang, G. J. Broussard, and L. Tian. Imaging chemical neurotransmission with genetically encoded fluorescent sensors. ACS Chem Neurosci, 6(1):84-93, 2015.

[49] S. Okumoto, L. L. Looger, K. D. Micheva, R. J. Reimer, S. J. Smith, and W. B. Frommer. Detection of glutamate release from neurons by genetically encoded surfacedisplayed fret nanosensors. Proc Natl Acad Sci USA, 102(24):8740-8745, 2005.

[50] M. A. Brun, K. T. Tan, E. Nakata, M. J. Hinner, and K. Johnsson. Semisynthetic 
fluorescent sensor proteins based on self-labeling protein tags. $J$ Am Chem Soc, 131(16):5873-5884, 2009.

[51] A. Masharina, L. Reymond, D. Maurel, K. Umezawa, and K. Johnsson. A fluorescent sensor for gaba and synthetic gaba(b) receptor ligands. J Am Chem Soc, 134(46):19026-19034, 2012.

[52] Alberto Schena and Kai Johnsson. Sensing acetylcholine and anticholinesterase compounds. Angew. Chem. Int. Ed., 53:1302-1305, 2014.

[53] Matthias A. Brun, Kui-Thong Tan, Rudolf Griss, Anna Kielkowska, Luc Reymond, and Kai Johnsson. A semisynthetic fluorescent sensor protein for glutamate. J. Am. Chem. Soc, 134:7676-7678, 2012.

[54] Sebastian Kruss, Markita P. Landry, Emma Vander Ende, Barbara M.A. Lima, Nigel F. Reuel, Jingqing Zhang, Justin Nelson, Bin Mu, Andrew Hilmer, and Michael Strano. Neurotransmitter detection using corona phase molecular recognition on fluorescent single-walled carbon nanotube sensors. Journal of the American Chemical Society, 136(2):713-724, 2014. PMID: 24354436.

[55] E. Polo and S. Kruss. Impact of redox-active molecules on the fluorescence of polymerwrapped carbon nanotubes. J. Phys. Chem. C, 120:3061-3070, 2016.

[56] Sebastian Kruss, Daniel P. Salem, Lela Vukovic, Barbara Lima, Emma Vander Ende, Edward S. Boyden, and Michael S. Strano. High-resolution imaging of cellular dopamine efflux using a fluorescent nanosensor array. PNAS, 17(8):1789-1794, 2017.

[57] Florian A. Mann, Niklas Herrmann, Daniel Meyer, and Sebastian Kruss. Tuning selectivity of fluorescent carbon nanotube-based neurotransmitter sensors. Sensors, $17(7), 2017$.

[58] A.A. Boghossian, J. Zhang, P.W. Barone, N.F. Reuel, J.H. Kim, D.A. Heller, J.H. Ahn, A. J. Hilmer, A. Rwei, J.R. Arkalgud, C.T. Zhang, and M.S. Strano. Nearinfrared fluorescent sensors based on single-walled carbon nanotubes for life sciences applications,. Chem Sus Chem, 4(7):848-863, 2011.

[59] Z. Liu, S. Tabakman, and H. Dai K. Welsher. Carbon nanotubes in biology and medicine: in vitro and in vivo detection, imaging and drug delivery. Nano Res., $2(2): 85-120,2009$.

[60] Andrew M. Smith, Michael C. Mancini, and Shuming Nie. Second window for in vivo imaging. Nature Nanotechnology, 4:710-711, 2009.

[61] M.S. Dresselhaus and G. Dresselhaus. Carbon Nanotubes: Synthesis, Structure, Properties, and Applications. Springer Berlin Heidelberg, 2001. 
[62] Sebastian Kruss, Andrew J. Hilmer, Jingqing Zhang, Nigel F. Reuel, Bin Mu, and Michael S. Strano. Carbon nanotubes as optical biomedical sensors. Advanced Drug Delivery Reviews, 65(15):1933-1950, 2013.

[63] C. Gao, Z. Guo, J.H. Liu, and X.J. Huang. The new age of carbon nanotubes: an updated review of functionalized carbon nanotubes in electrochemical sensors. Nanoscale, 4(6):1948-1963, 2012.

[64] Z. Chen, X. Zhang, R. Yang, Z. Zhu, Y. Chen, and W. Tan. Single-walled carbon nanotubes as optical materials for biosensing. Nanoscale, 3(5):1949-1956, 2011.

[65] W. Yang, K.R. Ratinac, S.P. Ringer, P. Thordarson, J.J. Gooding, and F. Braet. Carbon nanomaterials in biosensors: should you use nanotubes or graphene? Angew. Chem. Int. Ed. Engl., 49(12):2114-2138, 2010.

[66] Nigel F. Reuel, Brittany Grassbaugh, Sebastian Kruss, J. Zachary Mundy, Cary Opel, Adebola O. Ogunniyi, Kamal Egodage, Ramon Wahl, Bernhard Helk, Jingqing Zhang, Z. Ilke Kalcioglu, Kevin Tvrdy, Darin O. Bellisario, Bin Mu, Steven S. Blake, Krystyn J. Van Vliet, J. Christopher Love, Karl Dane Wittrup, and Michael S. Strano. Emergent properties of nanosensor arrays: Applications for monitoring igg affinity distributions, weakly affined hypermannosylation, and colony selection for biomanufacturing. ACS Nano, 7(9):7472-7482, 2013. PMID: 23909808.

[67] S. Iijima. Helical microtubules of graphitic carbon. Nature, 354:56-58, 1991.

[68] S. Iijima and T. Ichihashi. Single-shell carbon nanotubes of 1-nm diameter. Nature, 363:603-605, 1991.

[69] L.V. Radushkevich and V.M. Lukyanovich. The structure of carbon forming in thermal decomposition of carbon monoxide on an iron catalyst. Russian J Phys Chem, 26:26, 88-95, 1952.

[70] Nicole Grobert. Carbon nanotubes - becoming clean. Materials Today, 10(1):28 - 35, 2007.

[71] Khalid Saeed and Ibrahim Khan. Carbon nanotubes-properties and applications: a review. Carbon letters, 14(3):131-144, 2013.

[72] Mukul Kumar. Carbon nanotube synthesis and growth mechanism. In Siva Yellampalli, editor, Carbon Nanotubes - Synthesis, Characterization, Applications, chapter 08. InTech, Rijeka, 2011.

[73] R. Jasti and C. R. Bertozzi. Progress and challenges for the bottom-up synthesis of carbon nanotubes with discrete chirality. Chem. Phys. Lett., 494:1-7, 2010. 
[74] Sergei M. Bachilo, Leandro Balzano, Jose E. Herrera, Francisco Pompeo, Daniel E. Resasco, and R. Bruce Weisman. Narrow (n,m)-distribution of single-walled carbon nanotubes grown using a solid supported catalyst. Journal of the American Chemical Society, 125(37):11186-11187, 2003. PMID: 16220926.

[75] Pavel Nikolaev. Gas-phase production of single-walled carbon nanotubes from carbon monoxide: a review of the hipco process. Journal of Nanoscience and Nanotechnology, 4(4):307-316, 2004.

[76] Jan Prasek, Jana Drbohlavova, Jana Chomoucka, Jaromir Hubalek, Ondrej Jasek, Vojtech Adam, and Rene Kizek. Methods for carbon nanotubes synthesis-review. J. Mater. Chem., 21:15872-15884, 2011.

[77] Claudia Backes, Udo Mundloch, Alexander Ebel, Frank Hauke, and Andreas Hirsch.

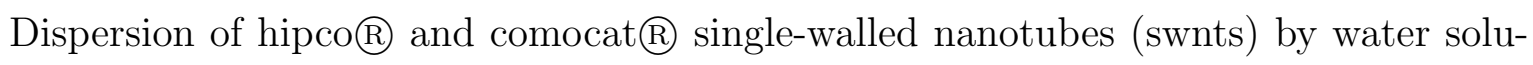
ble pyrene derivatives - depletion of small diameter swnts. Chemistry - A European Journal, 16(11):3314-3317, 2010.

[78] Ali Eatemadi, Hadis Daraee, Hamzeh Karimkhanloo, Mohammad Kouhi, Nosratollah Zarghami, Abolfazl Akbarzadeh, Mozhgan Abasi, Younes Hanifehpour, and Sang Woo Joo. Carbon nanotubes: properties, synthesis, purification, and medical applications. Nanoscale Res Lett., 9(1):393, 2014.

[79] Jeroen W. G. Wilder, Liesbeth C. Venema, Andrew G. Rinzler, Richard E. Smalley, and Cees Dekker. Electronic structure of atomically resolved carbon nanotubes. Nature, 391:59-62, 1998.

[80] Mei Zhang and Jian Li. Carbon nanotube in different shapes. Materials Today, 12(6):12 $-18,2009$.

[81] S. Reich, C. Thomsen, and J. Maultzsch. Carbon Nanotubes: Basic Concepts and Physical Properties. Wiley-VCH, 2003.

[82] Sebastien Nanot, Nicholas A. Thompson, Ji-Hee Kim, Xuan Wang, William D. Rice, Erik H. Haroz, Yogeeswaran Ganesan, Cary L. Pint, and Junichiro Kono. Springer Handbook of Nanomaterials: Single-Walled Carbon Nanotubes. Number 105-146. Springer Handbook, 2013.

[83] P. Avouris, M. Freitag, and Perebeinos V. Carbon-nanotube photonics and optoelectronics. Nat. Photonics, 2:341-350, 2008.

[84] M. S. Arnold, J. L. Blackburn, J. J. Crochet, S. K. Doorn, A. Duque, J. G.and Mohite, and H. Telg. Recent developments in the photophysics of single-walled carbon nan- 
otubes for their use as active and passive material elements in thin film photovoltaics. Phys. Chem. Chem. Phys., 15:14896-14918, 2013.

[85] A.R.T. Nugraha, R. Saito, K. Sato, P.T. Araujo, A. Jorio, and M.S. Dresselhaus. Dielectric constant model for environmental effects on the exciton energies of single wall carbon nanotubes. Appl. Phys. Lett., 97, 2010.

[86] Riichiro Saito and Hiromichi Kataura. Optical Properties and Raman Spectroscopy of Carbon Nanotubes. Part of the Topics in Applied Physics., volume 80. Springer-Verlag Berlin Heidelberg 2001, 2011.

[87] B.K. Kaushik and M.K. Majumder. Carbon Nanotube: Properties and Applications, volume 5. SpringerBriefs in Applied Sciences and Technology,, 232012.

[88] M. S. Dresselhaus, G. Chen, M. Y. Tang, R. G. Yang, H. Lee, D. Z. Wang, Z. F. Ren, J.P. Fleurial, and P. Gogna. New directions for low-dimensional thermoelectric materials. Advanced Materials, 19(8):1043-1053, 2007.

[89] Elena Bekyarova, Irina Kalinina, Mikhail E. Itkis, Leanne Beer, Nelson Cabrera, and Robert C. Haddon. Mechanism of ammonia detection by chemically functionalized single-walled carbon nanotubes. Journal of the American Chemical Society, 129(35):10700-10706, 2007. PMID: 17696430.

[90] Mildred S. Dresselhaus, Gene Dresselhaus, Riichiro Saito, and Ado Jorio. Exciton photophysics of carbon nanotubes. Annu. Rev. Phys. Chem., 58:719-747, 2007.

[91] S. Moritsubo, T. Murai, T. Shimada, Y. Murakami, S. Chiashi, S. Maruyama, and Y. K. Kato. Exciton diffusion in air-suspended single-walled carbon nanotubes. Phys. Rev. Lett., 104:247402, Jun 2010.

[92] Yuhei Miyauchi, Kazunari Matsuda, Yuki Yamamoto, Naotoshi Nakashima, and Yoshihiko Kanemitsu. Length-dependent photoluminescence lifetimes in single-walled carbon nanotubes. The Journal of Physical Chemistry C, 114(30):12905-12908, 2010.

[93] Jared J. Crochet, Juan G. Duque, James H. Werner, and Stephen K. Doorn. Photoluminescence imaging of electronic-impurity-induced exciton quenching in single-walled carbon nanotubes. Nature Nanotechnology, 7:126-132, 2012.

[94] M. L. Spaeth and W. R. Sooy. Fluorescence and bleaching of organic dyes. J of Chem Physics, 48(2315), 1968.

[95] Prakrit V. Jenaa, Thomas V. Galassia, Daniel Roxburyc, and Daniel A. Heller. Review. progress toward applications of carbon nanotube photoluminescence. ECS J. Solid State Sci. Technol., 6(6):3075-3077, 2017. 
[96] Jared Crochet, Michael Clemens, and Tobias Hertel. Quantum yield heterogeneities of aqueous single-wall carbon nanotube suspensions. J Am Chem Soc, 2007.

[97] Arko Graf, Yuriy Zakharko, Stefan P. Schiessl, Claudia Backes, Moritz Pfohl, Benjamin S. Flavel, and Jana Zaumseil. Large scale, selective dispersion of long singlewalled carbon nanotubes with high photoluminescence quantum yield by shear force mixing. Carbon, 105:593-599, 2016.

[98] Feng Wang, Gordana Dukovic, Louis E. Brus, and Tony F. Heinz. Time-resolved fluorescence of carbon nanotubes and its implication for radiative lifetimes. Physical Review Letters, 92(17):177401-177404, 2004.

[99] Jacques Lefebvre, David G. Austing, Jeffery Bond, and Paul Finnie. Photoluminescence imaging of suspended single-walled carbon nanotubes. Nano Letters, 6(8):16031608, 2006. PMID: 16895343.

[100] Sang-Yong Ju, William P. Kopcha, and Fotios Papadimitrakopoulos. Brightly fluorescent single-walled carbon nanotubes via an oxygen-excluding surfactant organizatio. Science, 323(5919):1319-1323, 2009.

[101] Andrea J. Lee, Xiaoyong Wang, Lisa J. Carlson, Julie A. Smyder, Bradford Loesch, Xiaomin Tu, Ming Zheng, and Todd D. Krauss. Bright fluorescence from individual single-walled carbon nanotubes. Nano Letters, 11(4):1636-1640, 2011. PMID: 21417364.

[102] Zhentao Hou and Todd D. Krauss. Photoluminescence brightening of isolated singlewalled carbon nanotubes. The Journal of Physical Chemistry Letters, 8(19):4954-4959, 2017. PMID: 28937225.

[103] S. Cambre, S. M. Santos, W. Wenseleers, A. R. T. Nugraha, R. Saito, L. Cognet, and B. Lounis. Luminescence properties of individual empty and water-filled single-walled carbon nanotubes. ASC Nano, American Chemical Society, 6(3):2649-2660, 2012.

[104] Yuhei Miyauchi, Munechiyo Iwamura, Shinichiro Mouri, Tadashi Kawazoe, Motoichi Ohtsu, and Kazunari Matsuda. Brightening of excitons in carbon nanotubes on dimensionality modification. Nature Photonics, 7:715-719, 2012.

[105] J. Ramirez, M.L. Mayo, S. Kilina, and S. Tretiak. Electronic structure and optical spectra of semiconducting carbon nanotubes functionalized by diazonium salts. Chemical Physics, 413:89-101, February 2013.

[106] Yanmei Piao, Brendan Meany, Lyndsey R. Powell, Nicholas Valley, Hyejin Kwon, George C. Schatz, and YuHuang Wang. Brightening of carbon nanotube photolu- 
minescence through the incorporation of sp3 defects. Nature Chemistry, 5:840-845, 2013.

[107] Anton V. Naumov, Saunab Ghosh, Dmitri A. Tsyboulski, Sergei M. Bachilo, and R. Bruce Weisman. Analyzing absorption backgrounds in single-walled carbon nanotube spectra. ACS Nano, 5(3):1639-1648, 2011. PMID: 21341755.

[108] Sergei M. Bachilo, Michael S. Strano, Carter Kittrell, Robert H. Hauge, Richard E. Smalley, and R. Bruce Weisman. Structure-assigned optical spectra of single-walled carbon nanotubes. Science, 298(5602):2361-2366, 2002.

[109] R. Saito, G. Dresselhaus, and M. S. Dresselhaus. Trigonal warping effect of carbon nanotubes. Phys. Rev. B, 61:2981-2990, Jan 2000.

[110] M. E. Itkis, D. E. Perea, S. Niyogi, S. M. Rickard, M. A. Hamon, B. Zhao, and R. C. Haddon. Purity evaluation of as-prepared single-walled carbon nanotube soot by use of solution-phase near-ir spectroscopy. Nano Lett., 3:309-314, 2003.

[111] K. L. Day and D. R. Huffman. Measured extinction efficiency of graphite smoke in region 1200-6000. Nat. Phys. Sci., 243:50-51, 1973.

[112] J-S. Lauret, C. Voisin, G. Cassabois, C. Delalande, Ph. Roussignol, O. Jost, and L. Capes. Ultrafast carrier dynamics in single-wall carbon nanotubes. Phys. Rev. Lett., 90:057404, Feb 2003.

[113] A. Nish, J. Y. Hwang, J. Doig, and R. J. Nicholas. Highly selective dispersion of singlewalled carbon nanotubes using aromatic polymers. Nat. Nanotechnol., 2:640-646, 2007.

[114] Laurent Cognet, Dmitri A. Tsyboulski, John-David R. Rocha, Condell D. Doyle, James M. Tour, and R. Bruce Weisman. Stepwise quenching of exciton fluorescence in carbon nanotubes by single molecule reactions. Science, 316(5830):1465-1468, 2007.

[115] Michael J. Connell, Sergei M. Bachilo, Chad B. Huffman, Valerie C. Moore, Michael S. Strano, Erik H. Haroz, Kristy L. Rialon, Peter J. Boul, William H. Noon, Carter Kittrell, Jianpeng Ma, Robert H. Hauge, R. Bruce Weisman, and Richard E. Smalley. Band gap fluorescence from individual single-walled carbon nanotubes. Science, 297(5581):593-596, 2002.

[116] Yan Yan Huang and Eugene M. Terentjev. Dispersion of carbon nanotubes: Mixing, sonication, stabilization, and composite properties. Polymers, 4(1):275-295, 2012.

[117] Michael S. Arnold, Alexander A. Green, James F. Hulvat, Samuel I. Stupp, and Mark C. Hersam. Sorting carbon nanotubes by electronic structure using density differentiation. Nature Nanotechnology, 1:60-65, 2006. 
[118] Scott M. Tabakman, Kevin Welsher, Guosong Hong, and Hongjie Dai. Optical properties of single-walled carbon nanotubes separated in a density gradient; length, bundling, and aromatic stacking effects. J Phys Chem C Nanomater Interfaces, 114(46):19569-19575, 2010.

[119] X. Sun, S. Zaric, D. Daranciang, K. Welsher, Y. Lu, X. Li, and H. Dai. Optical properties of ultrashort semiconducting single-walled carbon nanotube capsules down to sub-10 nm. Journal of the American Chemical Society., 130(20):6551-6555, 2008.

[120] Arnold M. S, Stupp S. I., and Hersam M. C. Enrichment of single-walled carbon nanotubes by diameter in density gradients. Nano Letters., 5(4):713-718, 2005.

[121] Takeshi Tanaka, Hehua Jin, Yasumitsu Miyata, Shunjiro Fujii, Hiroshi Suga, Yasuhisa Naitoh, Takeo Minari, Tetsuhiko Miyadera, Kazuhito Tsukagoshi, and Hiromichi Kataura. Simple and scalable gel-based separation of metallic and semiconducting carbon nanotubes. Nano Letters, 9(4):1497-1500, 2009. PMID: 19243112.

[122] Huaping Liu, Daisuke Nishide, Takeshi Tanaka, and Hiromichi Kataura. Large-scale single-chirality separation of single-wall carbon nanotubes by simple gel chromatography. Nature Communications, 2:309, 2011.

[123] Sang N. Kim, Zhifeng Kuang, James G. Grote, Barry L. Farmer, and Rajesh R. Naik. Enrichment of $(6,5)$ single wall carbon nanotubes using genomic dna. Nano Letters, 8(12):4415-4420, 2008. PMID: 19053794.

[124] J. Gao, M. Kwak, J. Wildeman, A. Hermann, M. A. Loi, and A. Herrmann. Effectiveness of sorting single-walled carbon nanotubes by diameter using polyfluorene derivatives. Carbon, 49(1):333-338, 1 2011. ISI:000284977500047.

[125] M.S. Dresselhaus, A. Jorio, and Saito R. Characterizing graphene and carbon nanotubes by raman spectroscopy. Annu. Rev. Condens. Matter Phys., 1:89-108, 2010.

[126] Christian Thomsen and Stephanie Reich. Raman scattering in carbon nanotubes, 01 1970.

[127] P.C. Eklund, J.M. Holden, and R.A. Jishi. Vibrational modes of carbon nanotubes; spectroscopy and theory. Carbon, 33(7):959 - 972, 1995. Nanotubes.

[128] Panayiotis Bilalis, Dimitrios Katsigiannopoulos, Apostolos Avgeropoulos, and Georgios Sakellariou. Non-covalent functionalization of carbon nanotubes with polymers. $R S C$ Adv., 4:2911, 2014.

[129] Tsuyohiko Fujigaya and Naotoshi Nakashima. Non-covalent polymer wrapping of carbon nanotubes and the role of wrapped polymers as functional dispersants. Sci. Technol. Adv. Mater., 16:21, 2015. 
[130] Valeria Ettorre Antonello Di Crescenzo and Antonella Fontana. Non-covalent and reversible functionalization of carbon nanotubes. Beilstein J. Nanotechnol., 5:16751690, 2014.

[131] N. Karousis, N. Tagmatarchis, and D. Tasis. Current progress on the chemical modification of carbon nanotubes,. Chem. Rev., 110(9):5366-5397, 2010.

[132] P. Singh, S. Campidelli, S. Giordani, D. Bonifazi, A. Bianco, and M. Prato. Organic functionalisation and characterisation of single-walled carbon nanotubes,. Chem. Soc. Rev., 38(8):2214-2230, 2009.

[133] Z. Liu, S.M. Tabakman, Z. Chen, and H. Dai. Preparation of carbon nanotube bioconjugates for biomedical applications. Nat. Protoc., 4(9):1372-1382, 2009.

[134] Michael S. Strano, Valerie C. Moore, Michael K. Miller, Mathew J. Allen, Erik H. Haroz, Carter Kittrell, Robert H. Hauge, and R. E. Smalley. The role of surfactant adsorption during ultrasonication in the dispersion of single-walled carbon nanotubes. Journal of Nanoscience and Nanotechnology, 3(1-2):81-86, 2003.

[135] Pu Chun Ke. Fiddling the string of carbon nanotubes with amphiphiles. Phys. Chem. Chem. Phys., 9:439-447, 2007.

[136] WJ Pape and U Hoppe. Standardization of an in vitro red blood cell test for evaluating the acute cytotoxic potential of tensides. Arzneimittel-Forschung, 40(4):498 — 502, April 1990.

[137] Y. Kato, A. Inoue, Y. Niidome, and N. Nakashima. Thermodynamics on soluble carbon nanotubes: How do dna molecules replace surfactants on carbon nanotubes? Scientific Reports, 2:733, 2012.

[138] Sara Iliafar, Jeetain Mittal, Dmitri Vezenov, and Anand Jagota. Interaction of single-stranded dna with curved carbon nanotube is much stronger than with flat graphite. Journal of the American Chemical Society, 136(37):12947-12957, 2014. PMID: 25162693.

[139] Z. Liu, S. Tabakman, and H. Dai K. Welsher. Carbon nanotubes in biology and medicine: in vitro and in vivo detection, imaging and drug delivery. Nano Res., $2(2): 85-120,2009$.

[140] Z. Chen, X. Zhang, R. Yang, Z. Zhu, Y. Chen, and W. Tan. Single-walled carbon nanotubes as optical materials for biosensing. Nanoscale, 3(5):1949-1956, 20111.

[141] R. Tang, Y. Shi, Z. Hou, and L. Wei. Carbon nanotube-based chemiresistive sensors. Sensors, 17(4):882, 2017. 
[142] Otto S. Wolfbeis. Probes, sensors, and labels: Why is real progress slow? Angewandte Chemie International Edition, 52:9864-9865, 2013.

[143] Ken Donaldson, Craig A. Poland, Fiona A. Murphy, Marion MacFarlane, Tatyana Chernova, and Anja Schinwald. Pulmonary toxicity of carbon nanotubes and asbestos — similarities and differences. Advanced Drug Delivery Reviews, 65(15):2078 - 2086, 2013. Carbon Nanotubes in Medicine and Biology: Therapy and Diagnostics \& Safety and Toxicology.

[144] M. C.-F. Jaurand, A. Renier, and J. Daubriac. Mesothelioma: Do asbestos and carbon nanotubes pose the same health risk? Particle and Fibre Toxicology., 6(16), 2009.

[145] S.Y. Madani, A. Mandel, and A. M. Seifalian. A concise review of carbon nanotubes toxicology. Nano Reviews., 4, 2013.

[146] N. Kobayashi, H. Izumi, and Y. Morimoto. Review of toxicity studies of carbon nanotubes. Journal of Occupational Health., 59(5):394-407, 2017.

[147] Chiu-Wing Lam, John James, Richard McCluskey, Sivaram Arepalli, and Robert Hunter. A review of carbon nanotube toxicity and assessment of potential occupational and environmental health risks. Crit Rev Toxicol., 36:189-217, 042006.

[148] Antonio Pietroiusti, Micol Massimiani, Ivana Fenoglio, Massimiliano Colonna, Federica Valentini, Giuseppe Palleschi, Antonella Camaioni, Andrea Magrini, Gregorio Siracusa, Antonio Bergamaschi, Alessandro Sgambato, and Luisa Campagnolo. Low doses of pristine and oxidized single-wall carbon nanotubes affect mammalian embryonic development. ACS Nano, 5(6):4624-4633, 2011. PMID: 21615177.

[149] Ken Donaldson and Craig A. Poland. Multiwalled carbon nanotubes inhaled by mice can reach the outer lining of the lungs and cause scarring. Nature Nanotechnology, 4:708-710, 2009.

[150] C. Ge, J. Du, L. Zhao, L. Wang, Y. Liu, D. Li, Y. Yang, R. Zhou, Y. Zhao, and C. Chai, Z. amd Chen. Binding of blood proteins to carbon nanotubes reduces cytotoxicity. C.Proc. Natl. Acad. Sci., 108(41):16968-16973, 2011.

[151] Alessandra Antonucci, Justyna Kupis-Rozmysłowicz, and Ardemis A. Boghossian. Noncovalent protein and peptide functionalization of single-walled carbon nanotubes for biodelivery and optical sensing applications. ACS Appl. Mater. Interfaces, 9:11321$11331,2017$.

[152] Zhuang Liu, Corrine Davis, Weibo Cai, Lina He, Xiaoyuan Chen, and Hongjie Dai. Circulation and long-term fate of functionalized, biocompatible single-walled carbon 
nanotubes in mice probed by raman spectroscopy. Proceedings of the National Academy of Sciences, 105(5):1410-1415, 2008.

[153] Meike L. Schipper, Nozomi Nakayama-Ratchford, Corrine R. Davis, Nadine Wong Shi Kam, Pauline Chu, Zhuang Liu, Xiaoming Sun, Hongjie Dai, and Sanjiv S. Gambhir. A pilot toxicology study of single-walled carbon nanotubes in a small sample of mice. Nature Nanotechnology, 3:216-221, 2008.

[154] J. Zhang, A.A. Boghossian, P.W. Barone, A. Rwei, J.-H. Kim, D. Lin, D.A. Heller, A.J. Hilmer J.H. Hilmer, N. Nair, N.F. Reuel, and M.S. Strano. Single molecule detection of nitric oxide rnabled by d(at) 15 dna adsorbed to near infrared fluorescent single-walled carbon nanotubes. J. Am. Chem. Soc., 133(3):567-581., 2010.

[155] J.H. Kim, D.A. Heller, H. Jin, P.W. Barone, C. Song, J. Zhang, L.J. Trudel, G.N. Wogan, S.R. Tannenbaum, and M.S. Strano. The rational design of nitric oxide selectivity in single-walled carbon nanotube near-infrared fluorescence sensors for biological detection. Nat. Chem., 1(6):473-481, 2009.

[156] T.G. Cha, B.A. Baker, M.D. Sauffer, J. Salgado, D. Jaroch, J.L. Rickus, D.M. Porterfield, and J.H. Choi. Optical nanosensor architecture for cell-signaling molecules using dna aptamer-coated carbon nanotubes. ASC Nano, 5(5):4236-4244, 2011.

[157] J. Garthwaite and C.L. Boulton. Nitric-oxide signaling in the central-nervous-system. Annu. Rev. Physiol., 57:683-706, 1995.

[158] J.P. Rivot, J. Barraud, C. Montecot, B. Jost, and J.M. Besson. Nitric oxide (no): in vivo electrochemical monitoring in the dorsal horn of the spinal cord of the rat. Brain Res., 773(1):66-75, 1997.

[159] E.S. Jeng, A.E. Moll, A.C. Roy, J.B. Gastala, and M.S. Strano. Detection of dna hybridization using the near-infrared band-gap fluorescence of single-walled carbon nanotubes. Nano Lett., 6(3):371-375, 2006.

[160] E.S. Jeng, P.W. Barone, J.D. Nelson, and M.S. Strano. Hybridization kinetics and thermodynamics of dna adsorbed to individually dispersed single-walled carbon nanotubes. Small, 3(9):1602-1609, 2007.

[161] M. E. Lynge, R. van der Westen, A. Postma, and B. Stadler. Polydopamine - a nature-inspired polymer coating for biomedical science. Nanoscale, 3:4916, 2011.

[162] H. Lee, S. M. Dellatore, W. M. Miller, and P. B. Messersmith. Mussel-inspired surface chemistry for multifunctional coatings. Science, 318(426), 2007.

[163] J. D. Andrade, V. Hlady, and A. P. Wei. Adsorption of complex proteins at interfaces. Pure Appl. Chem., 64(11):1777-1781, 1992. 
[164] S. S. Karajanagi, H. Yang, P. Asuri, E. Sellitto, J. S. Dordick, and R. S. Kane. Proteinassisted solubilization of single-walled carbon nanotubes. Langmuir, 22(4):1392-1395, 2006.

[165] J. Hilding, E. A. Grulke, Z. G. Zhang, and F. Lockwood. Dispersion of carbon nanotubes in liquids. J. Dispersion Sci. Technol., 24(1):1-41, 2003.

[166] D. Nepal and K. E. Geckeler. Proteins and carbon nanotubes: Close encounter in water. Small, 3(7):1259-1265, 2007.

[167] S. S. Karajanagi, A. A. Vertegel, R. S. Kane, and J. S. Dordick. Structure and function of enzymes adsorbed on single-walled carbon nanotubes. Langmuir, 20(10):1159411599, 2004.

[168] S. Az'hari and Y. Ghayeb. Effect of chirality, length and diameter of carbon nanotubes on the adsorption of 20 amino acids: A molecular dynamics simulation study. Mol. Simul., 40(5):392-398, 2014.

[169] P. Asuri, S. S. Karajanagi, H. Yang, T. J. Yim, R. S. Kane, and J. S. Dordick. Increasing protein stability through control of the nanoscale environment. Langmuir, 22(13):5833-5836, 2006.

[170] D. Nepal and K. E. Geckeler. Ph-sensitive dispersion and debundling of single-walled carbon nanotubes: Lysozyme as a tool. Small, 2(3):406-412, 2006.

[171] H. Yoon, J.H. Ahn, P.W. Barone, K. Yum, R. Sharma, A.A. Boghossian, J.H. Han, and M.S. Strano. Periplasmic binding proteins as optical modulators of single-walled carbon nanotube fluorescence: amplifying a nanoscale actuator. Angew. Chem. Int. Ed., 50(8):1828-1831, 2011.

[172] P.W.S. Barone. Single walled carbon nanotubes as reporters for the optical detection of glucose. J. Diabetes Sci. Technol., 3(2):11, 2009.

[173] Christian van der Does and R. Tampe. How do abc transporters drive transport? Biol. Chem., 385(10):972-933, 2004.

[174] L. Tolosa and G. Rao. Glucose Sensing. Topics in Fluorescence Spectroscopy,, volume 11. Springer, Boston, MA, 2006.

[175] M. A. Dwyer and H. W. Hellinga. Periplasmic binding proteins: A versatile superfamily for protein engineering. Curr. Opin. Struct. Biol., 14(4):495-504, 2004.

[176] R. O. Hynes and Q. Zhao. The evolution of cell adhesion. J Cell Biol., 150:89-96, 2000. 
[177] Benjamin Geiger, Joachim P. Spatz, and Alexander D. Bershadsky. Environmental sensing through focal adhesions. Nature Reviews Molecular Cell Biology, 10:21-33, 2009.

[178] C. H. Streuli. Integrins as architects of cell behavior. Mol. Biol. Cell, 27(19):2885-2888, 2016.

[179] J. S. Bennett. Structure and function of the platelet integrin alphaiibbeta3. J. Clin. Invest., 115(12):3363-3369, 2005.

[180] R. O. Hynes. Integrins: Bidirectional, allosteric signaling machines. Cell, 110, 2002.

[181] Iain D. Campbell and Martin J. Humphries. Integrin structure, activation, and interactions. Cold Spring Harb Perspect Biol, 19(49), 2011.

[182] Kun Zhang and JianFeng Chen. The regulation of integrin function by divalent cations. Cell Adhesion and Migration, 6(1), 2012.

[183] B. Nieswandt, D. Varga-Szabo, and M. Elvers. Integrins in platelet activation. J. Thromb. Haemost., 7(1):206-209, 2009.

[184] P. A. Gurbel, Y. H. Jeong, E. P. Navarese, and U. S. Tantry. Platelet-mediated thrombosis: From bench to bedside. Circ. Res., 118(9):1380-1391, 2016.

[185] K. Ley, J. Rivera-Nieves, W. J. Sandborn, and S. Shattil. Integrin-based therapeutics: biological basis, clinical use and new drugs biological basis, clinical use and new drugs. Nat. Rev. Drug Discov., 15:173-183, 2016.

[186] Carlos Mas-Moruno, Florian Rechenmacher, and Horst Kessler. Cilengitide: The first anti-angiogenic design, synthesis and clinical evaluation. Anti-Cancer Agents in Medicinal Chemistry, 10:753-768, 2010.

[187] Masamichi Nagae, Suyong Re, Emiko Mihara, Terukazu Nogi, Yuji Sugita, and Junichi Takagi. Crystal structure of integrin ectodomain: Atomic details of the fibronectin. The Journal of Cell Biology, 197(1):131-140, 2012.

[188] M. D. Pierschbacher and Ruoslaht E. Cell attachment activity of fibronectin can be duplicated by small synthetic fragments of the molecule. Nature, 309:30-33, 1984.

[189] Markus Nieberler, Ute Reuning, Florian Reichart, Johannes Notni, Hans-Juergen Wester, Markus Schwaiger, Michael Weinmueller, Andreas Raeder, Katja Steiger, and Horst Kessler. Exploring the role of rgd-recognizing integrins in cancer. Cancers, 9(9), 2017.

[190] Tobias G. Kapp, Florian Rechenmacher, Stefanie Neubauer, Oleg V. Maltsev, Elisabetta A. Cavalcanti-Adam, Revital Zarka, Ute Reuning, Johannes Notni, Hans-Juergen 
Wester, Carlos Mas-Moruno, Joachim Spatz, Benjamin Geiger, and Horst Kessler. Comprehensive evaluation of the activity and selectivity profile of ligands for rgdbinding integrins. Sci. Rep., 7:39805, 2017.

[191] G. Mueller, M. Gurrath, and H. Kessler. Pharmacophore refinement of gpiib and iiia antagonists based on comparative studies of antiadhesive cyclic and acyclic rgd peptides. J. Comp. Aided Mol., 8:709-730, 1994.

[192] M. Pfaff, K Tangemann, B. Mueller, M. Gurrath, G. Mueller, H. Kessler, R. Timpl, and J Engel. Selective recognition of cyclic rgd peptides of nmr defined conformation. J Biol Chem., 269(32):20233-20238, 1994.

[193] T. Weide, A. Modlinger, and H. Kessler. Spatial screening for the identification of the bioactive conformation of integrin ligands. Topics in Current Chemistry, 272:1-50, 2007.

[194] A. V. Naumova, M. Modo, A. Moore, C. E. Murry, and J. A. Frank. Clinical imaging in regenerative medicine. Nat. Biotechnol., 32:804-818, 2014.

[195] E. E. Hoover and J. A. Squier. Advances in multiphoton microscopy technology. Nat. Photon., 7:93-101, 2013.

[196] Guosong Hong, Alexander L. Antaris, and Hongjie Dai. Near-infrared fluorophores for biomedical imaging. Nature Biomedical Engineering, 1, 2017.

[197] Peter Kapusta, Michael Wahl, Ales Benda, Martin Hof, and Joerg Enderlein. Fluorescence lifetime correlation spectroscopy. J Fluoresc., 17(1):43-48, 2007.

[198] H. Inaba, M. Toida, M. Kondo, and T. Ichimura. Laser Computed Tomographic Bioimaging by Means of Coherent Detection Imaging. Springer, Berlin, Heidelberg, 1992.

[199] P. W. Barone, R. S. Parker, and M. S. Strano. In vivo fluorescence detection of glucose using a single-walled carbon nanotube optical sensor: design, fluorophore properties, advantages, and disadvantages. Anal. Chem., 7(7556), 2005.

[200] S. Johnsen. Hidden in plain sight: the ecology and physiology of organismal transparency. Biol. Bull., 201:301-318, 2001.

[201] David J. Lockwood. Rayleigh and Mie Scattering. Springer Berlin Heidelberg, Berlin, Heidelberg, 2014.

[202] Charles S. Johnson Jr. and Don A. Gabriel. Laser Light Scattering. Dover Publications, 1995. 
[203] Nicholas G. Horton, Ke Wang, Demirhan Kobat, Catharine G. Clark, Frank W. Wise, Chris B. Schaffer, and Chris Xu. In vivo three-photon microscopy of subcortical structures within an intact mouse brain. Nat. Photon., 7:205-209, 2013.

[204] A. N. Bashkatov, E. A. Genina, and V. V. Tuchin. Optical properties of skin, subcutaneous, and muscle tissues: a review. J. Innov. Opt. Health Sci., 4:9-38, 2011.

[205] A. N. Bashkatov, E. A. Genina, V. I. Kochubey, and V. V. Tuchin. Optical properties of human skin, subcutaneous and mucous tissues in the wavelength range from 400 to 2000 nm. Journal of Physics D, 38(15):2543-2555, 2005.

[206] M. Friebel, J. Helfmann, U. Netz, and M. Meinke. Influence of oxygen saturation on the optical scattering properties of human red blood cells in the spectral range 250 to 2000 nm. J Biomed Opt, 14(3), 2009.

[207] M. S. Viegas, T. C. Martins, F. Seco, and A. do Carmo. An improved and cost-effective methodology for the reduction of autofluorescence in direct immunofluorescence studies on formalin-fixed paraffin-embedded tissues. Eur. J. Histochem., 51:59-66, 2007.

[208] J. R. Lakowicz. Principles of Fluorescence Spectroscopy. Springer US, 2013.

[209] B Wozniak. Light Absorption in Sea Water. Springer, 2007.

[210] Kevin Welsher, Zhuang Liu, Sarah P. Sherlock, Joshua Tucker Robinson, Zhuo Chen, Dan Daranciang, and Hongjie Dai. A route to brightly fluorescent carbon nanotubes for near-infrared imaging in mice. Nature Nanotechnology, 4:773-780, 2009.

[211] Keitaro Umezawa, Daniel Citterio, and Koji Suzuki. New trends in near-infrared fluorophores for bioimaging. Analytical Sciences, 30(3):327-349, 2014.

[212] M. Ogawa, N. Kosaka, P.L. Choyke, and H. Kobayashi. In vivo molecular imaging of cancer with a quenching near-infrared fluorescent probe using conjugates of monoclonal antibodies and indocyanine green. Cancer Res., 69(4):1268-1272., 2009.

[213] M.Y. Berezin, W.J. Akers, K. Guo, G.M. Fischer, E. Daltrozzo, A. Zumbusch, and S. Achilefu. Long fluorescence lifetime molecular probes based on near infrared pyrrolopyrrole cyanine fluorophores for in vivo imaging. Biophys J., 97(9):22-24, 2009.

[214] Y. Ye, S. Bloch, B. Xu, and S. Achilefu. Design, synthesis, and evaluation of near infrared fluorescent multimeric rgd peptides for targeting tumors. J Med Chem., 49(7), 2006.

[215] Gianluca Accorsi, Giovanni Verri, Margherita Bolognesi, Nicola Armaroli, Catia Clementi, Costanza Miliani, and Aldo Romani. The exceptional near-infrared lumines- 
cence properties of cuprorivaite (egyptian blue). Chem. Commun., pages 3392-3394, 2009.

[216] Pablo García-Fernández, Miguel Moreno, and José Antonio Aramburu. Origin of the anomalous color of egyptian and han blue historical pigments: Going beyond the complex approximation in ligand field theory. J. Chem. Educ., 93:111-117, 2016.

[217] Heinz Berke. Chemistry in ancient times: The development of blue and purple pigments. Angew. Chem. Int. Ed, 41(14):2483-2487, 2002.

[218] J. Chaptal. Notice sur quelques couleurs trouvees a pompeia. Ann. Chim., 70(22), 1809.

[219] Heinz Berke. The invention of blue and purple pigments in ancient times. Chem. Soc. Rev., 36:15-30, 2007.

[220] M.; Aramburu J. A. García-Ferná ndez, P.; Moreno. Origin of the exotic blue colour of copper-containing historical pigments. Inorg. Chem., 54:192-199, 2015.

[221] Ya-Jie Li, Shi Ye, Chun-Hai Wang, Xiao-Ming Wang, and Qin-Yuan Zhang. Temperature-dependent near-infrared emission of highly concentrated cu2+ in cacusi4o10 phosphor. J. Mater. Chem. C, 2:10395-10402, 2014.

[222] R. M. Hazen and C. W. Burnham. The crystal structures of gillespite i and ii: A structure determination at high pressure. Am. Mineral., 59:1166-1176, 1974.

[223] Darrah Johnson-McDaniel, Christopher A. Barrett, Asma Sharafi, and Tina T. Salguero. Nanoscience of an ancient pigment. J. Am. Chem. Soc., 135:1677-1679, 2013.

[224] Weibo Chen, Yeqi Shi, Zhi Chen, Xiangwen Sang, Shuhong Zheng, Xiaofeng Liu, and Jianrong Qiu. Near-infrared emission and photon energy upconversion of twodimensional copper silicates. J. Phys. Chem. C, 119:20571-20577, 2015.

[225] Giorgio Pozza, David Ajjo, Giacomo Chiari, Franco De Zuane, and Marialuisa Favaro. Photoluminescence of the inorganic pigments egyptian blue, han blue and han purple.

J. Cult. Heritage, 1:393-398, 2000.

[226] Daniela Comelli, Valentina Capogrosso, Christian Orsenigo, and Austin Nevin. Dual wavelength excitation for the time-resolved photoluminescence imaging of painted ancient egyptian objects. Heritage Science, 4(21), 2016.

[227] B. A. Williams and J. C. Chaput. Synthesis of peptide-oligonucleotide conjugates using a heterobifunctional crosslinker. Curr Protoc Nucleic Acid Chem, 4:41-46, 2010. 
[228] G. Bisker, J. Ahn, Z.W. Kruss, S.and Ulissi, D.P. Salem, and M.S. Strano. A mathematical formulation and solution of the cophmore inverse problem for helically wrapping polymer corona phases on cylindrical substrates. J. Phys. Chem. C, 119:13876-13886, 2015.

[229] T. Hertel, S. Himmelein, T. Ackermann, D. Stich, and J. Crochet. Diffusion limited photoluminescence quantum yields in 1-d semiconductors: Single-wall carbon nanotubes. ACS Nano, 4:7161-7168, 2010.

[230] Fiona E. Harrison, Gene L. Bowman, and Maria Cristina Polidori. Ascorbic acid and the brain: Rationale for the use against cognitive decline. Nutrients, 6(4):1752-1781, 2014.

[231] Fiona E. Harrison and James M. May. Vitamin c function in the brain: Vital role of the ascorbate transporter (svct2). Free radical biology and medicine, 46(6):719-730, 2009.

[232] Cyrille Richard, Fabrice Balavoine, Patrick Schultz, Thomas W. Ebbesen, and Charles Mioskowski. Supramolecular self-assembly of lipid derivatives on carbon nanotubes. Science, 300(5620):775-778, 2003.

[233] M. F. Islam, E. Rojas, D. M. Bergey, A. T. Johnson, and A. G. Yodh. High weight fraction surfactant solubilization of single-wall carbon nanotubes in water. Nano Letters, $3(2): 269-273,2003$.

[234] Arko Graf, Yuriy Zakharko, Stefan P. Schießl, Claudia Backes, Moritz Pfohl, Benjamin S. Flavel, and Jana Zaumseil. Large scale, selective dispersion of long singlewalled carbon nanotubes with high photoluminescence quantum yield by shear force mixing. Carbon, 105:593 - 599, 2016.

[235] S. Steenken and S. V. Jovanovic. How easily oxidizable is dna? one-electron reduction potentials of adenosine and guanosine radicals in aqueous solution. J. Am. Chem. Soc., 119:617-618, 1997.

[236] Y. Hirana, G. Juhasz, Y. Miyauchi, S. Mouri, K. Matsuda, and N. Nakashima. Empirical prediction of electronic potentials of single- walled carbon nanotubes with a specific chirality (n,m). Sci. Rep., 3(2959), 2013.

[237] Y. Tanaka, Y. Hirana, Y. Niidome, K. Kato, S. Saito, and N. Nakashima. Experimentally determined redox potentials of individual $(n, m)$ single-walled carbon nanotubes. Angew. Chem., Int. Ed., 48:7655-7659, 2009.

[238] F. Sen, A. A. Boghossian, S. Sen, Z. W. Ulissi, J. Zhang, and M. S. Strano. Observation 
of oscillatory surface reactions of riboflavin, trolox, and singlet oxygen using single carbon nanotube fluorescence spectroscopy. ACS Nano, 6(10632), 2012.

[239] C. Y. Chen and C. T. Jafvert. Photoreactivity of carboxylated single-walled carbon nanotubes in sunlight: Reactive oxygen species production in water. Environ. Sci. Technol., 44:6674-6679, 2010.

[240] P. Boldog, K. Hajdu, M. Magyar, E. Hideg, and K. Hernadi. Carbon nanotubes quench singlet oxygen generated by photo-synthetic reaction centers. Phys. Status Solidi B, 250:2539-2543, 2013.

[241] J. J. Crochet, J. G. Duque, J. H. Werner, and S. K. Doorn. Photoluminescence imaging of electronic-impurity-induced exciton quenching in single-walled carbon nanotubes. Nat. Nanotechnol., 7:126-132, 2012.

[242] D. A. Heller, H. Jin, B. M. Martinez, D. Patel, B. M. Miller, T. K. Yeung, P. V. Jena, C. Hobartner, T. Ha, and S. K. Silverman. Multimodal optical sensing and analyte specificity using single- walled carbon nanotubes. Nat. Nanotechnol., 4:114-120, 2009.

[243] Jong Hyun Choi and Michael S. Strano. Solvatochromism in single-walled carbon nanotubes. Appl. Phys. Lett., 90(223114), 2007.

[244] B. Mu, T. P. McNicholas, J. Zhang, A. J. Hilmer, Z. Jin, N. F. Reuel, J. H. Kim, K. Yum, and M. S. Strano. A structure-function relationship for the optical modulation of phenyl boronic acid-grafted, polyethylene glycol-wrapped single-walled carbon nanotubes. Journal of the American Chemical Society, 134(42):17620-17627, 2012.

[245] Bin Mu, Thomas P. McNicholas, Jingqing Zhang, Andrew J. Hilmer, Zhong Jin, Nigel F. Reuel, Jong Ho Kim, Kyungsuk Yum, and Michael S. Strano. A structurefunction relationship for the optical modulation of phenyl boronic acid-grafted, polyethylene glycol-wrapped single-walled carbon nanotubes. $J$ Am Chem Soc, 134:17620-17627, 2012.

[246] Yifan Xu, Paul R. Berger, Jai Cho, and Richard B. Timmons. Capacitance-voltage characterization of pulsed plasma polymerized allylamine dielectrics for flexible polymeric field effect transistors. Journal of electronic materials, 33(8), 2004.

[247] A. Attic. Acrylic acid - a summary of safety and handling. Basic Acrylic Monomer Manufacturers, Inc. 4thEdition, 2013.

[248] W. Huang and D. G. Levitt. Theoretical calculation of the dielectric constant of a bilayer membrane. Biophys J., 17(2):17, 1977.

[249] M. Uematsu. Statistic dielectric constant of water and steam. J. Phys. Chem., 9(15), 1980. 
[250] T. Matsui, Y. Kitagawa, M. Okumura, and Y. Shigeta. Accurate standard hydrogen electrode potential and applications to the redox potentials of vitamin c and nad/nadh. J. Phys. Chem. A, 119:369-376, 2015.

[251] F. Martin, B. Ebel, C. Rojas, P. Gervais, N. Cayot, and R. Cacho. Redox potential: Monitoring and role in development of aroma compounds, rheological properties and survival of oxygen sensitive strains during the manufacture of fermented dairy products. InTech, 3:73-94, 2013.

[252] S. Sen, F. Sen, A. A. Boghossian, J. Zhang, and M. S. Strano. Effect of reductive dithiothreitol and trolox on nitric oxide quenching of single-walled carbon nanotubes. 2013,. J. Phys. Chem. C, 117:593-602, 2013.

[253] J. Zhang, M. P. Landry, P. W. Barone, J. H. Kim, S. Lin, Z. W. Ulissi, D. Lin, B. $\mathrm{Mu}, \mathrm{A}$. A. Boghossian, and A. J. Hilmer. Molecular recognition using corona phase complexes made of synthetic polymers adsorbed on carbon nanotubes. Nat. Nanotechnol., 8:959-968, 2013.

[254] N. V. Kurnosov, V. S. Leontiev, A. S. Linnik, and V. A. Karachevtsev. Influence of cysteine doping on photoluminescence intensity from semiconducting single-walled carbon nanotubes. Chem. Phys. Lett., 623:51-54, 2015.

[255] H. Hartleb, F. Spath, and T. Hertel. Evidence for strong electronic correlations in the spectra of gate-doped single-wall carbon nanotubes. ACS Nano, 9:10461-10470, 2015.

[256] J. G. Duque, L. Oudjedi, J. J. Crochet, S. Tretiak, B. Lounis, S. K. Doorn, and L. . Cognet. Mechanism of electrolyte-induced brightening in single-wall carbon nanotubes. J. Am. Chem. Soc., 135:3379-3382, 2013.

[257] Sebastian Kruss, Tobias Wolfram, Raquel Martin, Stefanie Neubauer, Horst Kessler, and Joachim P. Spatz. Stimulation of cell adhesion at nanostructured teflon interfaces. Adv. Mater., 22(48):5499-5506, 2010.

[258] Sebastian Kruss, Vesna Srot, Peter A. van Aken, and Joachim P. Spatz. Au-ag hybrid nanoparticle patterns of tunable size and density on glass and polymeric supports. Langmuir, 28(2):1562-1568, 2012.

[259] Sebastian Kruss, Luise Erpenbeck, Katharina Amschler, Tabea A. Mundinger, Heike Boehm, Hans-Joachim Helms, Tim Friede, Robert K. Andrews, Michael P. Schoen, and Joachim P. Spatz. Adhesion maturation of neutrophils on nanoscopically presented platelet glycoprotein ibalpha. ACS Nano, 7(11):9984-9996, 20013.

[260] Z. Liu, W. Cai, L. He, N. Nakayama, K. Chen, X. Sun, X. Chen, and H. Dai. In vivo 
biodistribution and highly efficient tumour targeting of carbon nanotubes in mice. Nat Nanotechnol, 2(1):47-52, 2007.

[261] A. De la Zerda, C. Zavaleta, S. Keren, S. Vaithilingam, S. Bodapati, Z. Liu, J. Levi, B. R. Smith, T. J. Ma, O. Oralkan, Z. Cheng, X. Chen, H. Dai, B. T. Khuri-Yakub, and S. S. Gambhir. Carbon nanotubes as photoacoustic molecular imaging agents in living mice. Nat Nanotechnol, 3(9):557-62, 2008.

[262] Prakrit V. Jena, Mohammad M. Safaee, Daniel A. Heller, and Daniel Roxbury. Dnacarbon nanotube complexation affinity and photoluminescence modulation are independent. ACS Appl. Mater. Interfaces, 9:21397-21405, 2017.

[263] J. K. Streit, S. M. Bachilo, S. Ghosh, C. W. Lin, and R. B. Weisman. Directly measured optical absorption cross sections for structure-selected single-walled carbon nanotubes. Nano Lett, 14(3):1530-1536, 2014.

[264] Friedrich Schoeppler, Christoph Mann, Tilman C. Hain, Felix M. Neubauer, Giulia Privitera, Francesco Bonaccorso, Daping Chu, Andrea C. Ferrari, and Tobias Hertel. Molar extinction coefficient of single-wall carbon nanotubes. J. Phys. Chem. C, 115:14682-14686, 2011.

[265] Jennifer F. Campbell, Ingrid Tessmert, H. Holden Thorp, and Dorothy A. Erie. Atomic force microscopy studies of dna-wrapped carbon nanotube structure and binding to quantum dots. J. Am. Chem. Soc., 130(32):10648-10655, 2008.

[266] R. R. Johnson, A. Kohlmeyer, A. T. C. Johnson, and M. L. Klein. Free energy landscape of a dna-carbon nanotube hybrid using replica exchange molecular dynamics. Nano Lett., 9(2):4, 2009.

[267] Cora-Ann Schoenenberger, A. Zuk, G. M. Zinkl, Donna Kendall, and Karl S. Matlin. Integrin expression and localization in normal mdck cells and transformed mdck cells lacking apical polarity. Journal of Cell Science, 107, 1994.

[268] D. D. Joseph, P. Whitley, and A. D. Chalmers. The mdck variety pack: choosing the right strain. BMC Cell Biol., 12(43), 2011.

[269] S. Kruss, T. Wolfram, R. Martin, S. Neubauer, H. Kessler, and J. P. Spatz. Stimulation of cell adhesion at nanostructured teflon interfaces. Adv. Mater., 22(48):5499-5506, 2010.

[270] Sebastian Kruss, Luise Erpenbeck, Katharina Amschler, Tabea A. Mundinger, Heike Boehm, Hans-Joachim Helms, Tim Friede, Robert K. Andrews, Michael P. Schön, and Joachim P. Spatz. Adhesion maturation of neutrophils on nanoscopically presented platelet glycoprotein ibalpha. ACS Nano, 7(11):9984-9996, 2013. 
[271] Katharina Amschler, Luise Erpenbeck, Sebastian Kruss, and Michael P. Schön. Nanoscale integrin ligand patterns determine melanoma cell behavior. ACS Nano, 8(9):9113-9125, 2014.

[272] Darrah Johnson-McDaniel and Tina T. Salguero. Exfoliation of egyptian blue and han blue, two alkali earth copper silicate-based pigments. J Vis Exp., 86(51686), 2014.

[273] Yu Chen, Min Kan, Qiang Sun, and Puru Jena. Structure and properties of egyptian blue monolayer family: Xcusi4o10 ( $\mathrm{x}=\mathrm{ca}$, sr, and ba). J. Phys. Chem. Lett., 7:399405, 2016.

[274] Glen I. Redford and Robert M. Clegg. Polar plot representation for frequency-domain analysis of fluorescence lifetime. Journal of Fluorescence, 15(5):805-815, 2005.

[275] Sergey M. Borisov, Christian Würth, Ute Resch-Genger, and Ingo Klimant†. New life of ancient pigments: Application in high-performance optical sensing materials. Anal. Chem., 85:9371-9377, 2013.

[276] J. Alan Creighton and Desmond G. Eadon. Ultraviolet-visible absorption spectra of the colloidal metallic elements. J. Chem. Soc., Faraday Trans., 87:3881-3891, 1991.

[277] M. S. Usman, M. E. E. Zowalaty, K. Shameli, N. Zainuddin, Salama M., and N. A. Ibrahim. Synthesis, characterization, and antimicrobial properties of copper nanoparticles. Int J Nanomedicine, 8:4467-4479, 2013. 


\section{List of abbreviations}

\begin{tabular}{|c|c|}
\hline AFM & Atomic Force Microscopy \\
\hline DNA & Deoxyribonucleic Acid \\
\hline DLS & Dynamic Light Scattering \\
\hline EB & Egyptian Blue \\
\hline ELISA & Enzyme-linked Immunosorbent Assay \\
\hline FRET & Förster Resonance Energy Transfer \\
\hline GABA & $\gamma$-aminobutyric acid \\
\hline GO & Graphite Oxide \\
\hline NAD & Nicotinamide Adenine Dinucleotide \\
\hline NADH & Reduced Nicotinamide Adenine Dinucleotide \\
\hline nIR & near Infrared \\
\hline PAA & Polyacrylic acid \\
\hline PAH & Polyallylamine hydrochloride \\
\hline PBS & Phosphate Buffered Saline \\
\hline PDMS & Polydimethylsiloxan \\
\hline PL & Photoluminescence \\
\hline PL-PEG-1.5k & $\begin{array}{l}\text { 1,2-dimyristoyl-sn-glycero-3-phosphoethanolamine-N- } \\
\text { methoxy-(polyethylene glycol)- } 1500\end{array}$ \\
\hline PL-PEG-5k & $\begin{array}{l}\text { 1,2-dimyristoyl-sn-glycero-3-phosphoethanolamine-N- } \\
\text { methoxy-(polyethylene glycol)-5000 }\end{array}$ \\
\hline RGD & Tripeptide sequence, Arg-Gly-Asp \\
\hline RGE & Tripeptide sequence, Arg-Gly-Glu \\
\hline ROS & Reactive Oxygen Species \\
\hline SC & Sodium Cholate \\
\hline SDBS & Sodium Dodecylbenzene Sulfonate \\
\hline SDS & Sodium Dodecyl Sulfate \\
\hline SMCC & $\begin{array}{l}\text { Succinimidyl } 4 \text {-(N-maleimidomethyl)cyclohexane-1- } \\
\text { carboxylate }\end{array}$ \\
\hline SNAP & Self-labeling Protein \\
\hline ssDNA & Single-stranded Deoxyribonucleic Acid \\
\hline SWCNTs & Single-Walled Carbon Nanotubes \\
\hline trolox & $\begin{array}{l}\text { 6-hydroxy-2,5,7,8-tetramethylchroman-2-carboxylic } \\
\text { acid }\end{array}$ \\
\hline QD & Quantum Dot \\
\hline QY & Quantum Yield \\
\hline
\end{tabular}




\section{Curriculum Vitae}

\section{Personal Data}

Name: $\quad$ Elena Alexandra Polo

Birthday: 23.07.1987

Birthplace: Moscow

Nationality: German

\section{Education}

2002 - 2006 Wilheilm-Raabe-Schule (Abitur), Hannover

2007 - 2011 Studies of Chemistry (B.Sc.), Georg-August-Universität Göttingen Bachelor thesis under supervision of Prof. Andreas Janshoff.

2011 - 2013 Studies of Chemistry (M.Sc.) Georg-August Universität Göttingen Master thesis under supervision of Prof. Andreas Janshoff.

2015 - 2018 Ph.D. Studies, Georg-August-Universität Göttingen Under supervision of Dr. Sebastian Kruss.

\section{Publications}

- Polo, E.; Nitka, T. T.; Neubert, E.; Erpenbeck L.; Vuković, L.; Kruss S., 2018, Control of integrin affinity by confining RGD peptides on fluorescent carbon nanotubes. ACS Applied Materials \&5 Interfaces, (submitted).

- Kliesch, T.-T.; Dietz, J.; Turco, L.; Halder, P.; Polo, E.; Tarantola, M.; Jahn, R.; Janshoff, A., 2017, Membrane tension increases fusion efficiency of model membranes in the presence of SNAREs. Scientific Reports, 7(12070), DOI:10.1038/s41598-017$12348-\mathrm{w}$

- Polo, E.; Kruss S., 2016, Impact of Redox-Active Molecules on the Fluorescence of Polymer-Wrapped Carbon Nanotubes, Journal of Physical Chemistry C, DOI:10.1021/acs.jpcc.5b12183.

- Polo, E.; Kruss S., 2016, Nanosensors for neurotransmitters. Invited review article, Journal of Analytical and Bioanalytical Chemistry, 408(1), 2727-2741, DOI:10.1007/s00216-015-9160-x.

- Schäfer, E.; Aue, D.; Tarantola, M.; Polo, E.; Westendorf, C.; Oikawa, N.; Bodenschatz, E.; Geil, B.; Janshoff, A., 2013, Collective behavior of Dictyostelium discoideum monitored by impedance analysis. Communicative and Integrative Biology, 6(3), 1-4, DOI:10.4161/cib.23894

- Schäfer, E.; Tarantola, M.; Polo, E.; Westendorf, C.; Oikawa, N.; Bodenschatz, E.; 
Geil, B.; Janshoff, A., 2013, Chemotaxis of Dictyostelium discoideum: collective oscillation of cellular contacts. PLOS ONE, 8(1), DOI:10.1371/journal.pone.0054172. 
Hiermit versichere ich, dass ich die vorliegende Doktorarbeit selbständig verfasst und keine anderen als die angegebenen Quellen und Hilfsmittel benutzt habe.

Elena Alexandra Polo 
Die Auswahl der Bespiele aus Kapitel 1.2 orientiert sich an dem Übersichtartikel: E. Polo, S. Kruss, Nanosensors for neurotransmitters. Invited review article, Journal of Analytical and Bioanalytical Chemistry, 2015, 408(1), pp 2727-2741, DOI: 10.1007/s00216-015-9160-x

Die Ergebnisse und Textabschnitte aus Kapitel 4.1 wurden publiziert in: E. Polo, S. Kruss, Impact of Redox-Active Molecules on the Fluorescence of Polymer-Wrapped Carbon Nanotubes, J. Phys. Chem. C, 2016, 120(5), pp 3061-3070, DOI: 10.1021/acs.jpcc.5b12183 



\section{Danksagung}

An dieser Stelle möchte ich mich bei allen bedanken, die mir mit Rat und Tat zur Seite standen. Insbesondere gilt mein Dank:

- Dr. Sebastian Kruss für die Möglichkeit zur Durchführung dieser Doktorarbeit, die Bereitstellung der interessanten und immer aktuellen Themen, seinen Enthusiasmus bei allen neuen Fragestellungen und für die Betreuung der Arbeit.

- Den Mitgliedern des Thesis Komitees Prof. Dr. Jörg Enderlein und Prof. Dr. Höbartner für hilfreiche Diskussionen und Anregungen während der Treffen.

- Den weiteren Mitgliedern der Prüfungskommission für ihre Zeit und Interesse an dem Projekt.

- Prof. Dr. Andreas Janshoff für die Einführung in die Welt der Biophysikalischen Chemie und Prof. Dr. Burkhard Geil für seine moralische Unterstützung auf dem Weg.

- FCI und GGNB für die finanzielle und organisatorische Unterstützung während der Promotionszeit.

- Prof. Dr. Burkhard Schmidt (Experimentelle und Angewandte Mineralogie), der uns erlaubt hatte an dessen Raman Gerät zu messen und so unsere erste Veröffentlichung möglich gemacht hatte. Sowie Kooperationspartnern Dr. James Daniel und Sofia Elizarova (Molekulare Neurobiologie, MPI für Experimentelle Medizin) für die sehr spannende Arbeit mit neuronalen Zellen. Und Zellkulturmitarbeitern, Angela Rübeling und Beate Rissom aus AK Janshoff, die alle MDCKII Zellen bis zu Experimenten am Leben erhalten hatten.

- Bachelor und Master Studenten, und zwar Alexander Speinert für seinen Beitrag zum Ägyptisch Blau Projekt und der Phasenseparation von Nanotubes, sowie Sabrine Mischke für ihre theoretischen Arbeiten an stand-off detection und die ersten Anfänge in Richtung der Bakteriensensoren.

- Meinen AK-Kollegen für die gute Arbeitsatmosphäre. Vor allem gilt mein Dank Elsa für ihre Selbstaufopferung bei der Zellisolation und Daniel für seine großartige Matlab Sammlung und den Aufbau von dem nIR Setup. Auch allen weiteren Kollegen, die an dem immer wachsenden nIR Setup mitgemacht haben, sowie allen Mitgliedern der AK Kruss, Janshoff und Steinem für die vielen Geräteeinführungen und Filmeabende.

- Martin für Deine Unterstützung während dieser Zeit und Deine Liebe.

- Meinen Eltern für euere Liebe und für alles, das ihr für mich getan habt. 